\title{
COWORKER INFLUENCE UPON INDIVIDUAL INTERNALIZATION OF SAFETY
}

\section{By}

Jeff S. Jackson

A thesis submitted to the Faculty of Graduate and Postdoctoral Affairs in partial fulfillment of the requirements for the degree of

Doctor of Philosophy

in

Management

Carleton University

Ottawa, Ontario

(C)2017

Jeff S. Jackson 


\section{ABSTRACT}

What is the influence of coworkers upon the individual's internalization of safety? Despite it being recognized as one of the most pervasive determinants of individual behaviour, social influence upon individual safety motivation has largely been overlooked by safety studies. This study operationalized group level safety climate to focus on coworker influence - an alternative to the predominant group level leadership perspective.

This exploratory, qualitative study interviewed 55 workers across three safety critical industries: firefighting, whitewater raft guiding, and paramedicine. The interviews were combined with workplace visits and six group interviews, using a theoretical proposition guided research model. The semi-structured qualitative approach allowed for the nature of the unstudied relationship between coworker influence and individual safety values to emerge. The focus of the study was at the individual level.

This study found that there is significant complexity at the coworker level regarding safety; more so than the predominant hierarchical safety climate paradigm. The major theoretical contribution of this research is a finding that coworker influence and social identity were an active influence upon internalizing safety motivation. Shared experience, social bonding and trust allowed for the individual to socially identify with their group and internalize group safety priorities and incorporate these into self-concept. Coworker social support and trust validated the individuals' sense of competency. This sense of competency was the first defense in dealing with work ambiguity, but more existentially, coworker endorsement of competency also anchored basic work identity. Safety at its most concrete level was about not getting hurt. Safety at its more meaningful level was about the potential harm from finding one's self on the outside of the work group and the internal or psychological implications of such exposure.

This research adds complexity to the field of safety studies by including peer interaction and internal safety values. This research also introduces to safety studies as-yet underutilized social theories as a means of examining coworker influence and internalizing safety. In particular, self-determination theory and social identification theory are applied to group and individual level safety behaviour. 


\section{ACKNOWLEDGEMENTS}

I wish to thank my Supervisor, Dr. Steven Murphy, for his work in seeing this project through. He seemed to know just the right questions to ask.

I also wish to recognize the contribution of my thesis committee, and in particular, Dr. Linda Schweitzer, who offered guidance at key points. 


\section{TABLE OF CONTENTS}

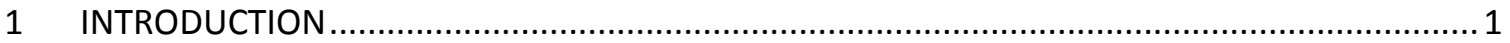

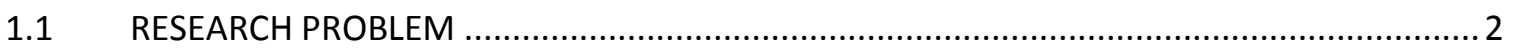

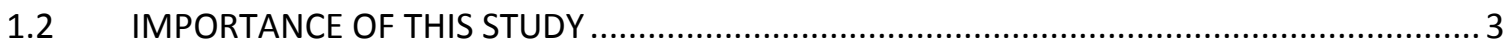

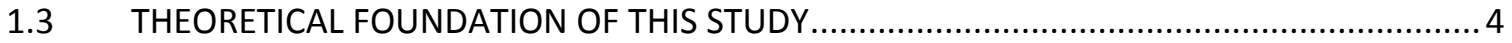

$1.4 \quad$ VARIABLES UNDER STUDY AND RESEARCH APPROACH …........................................ 6

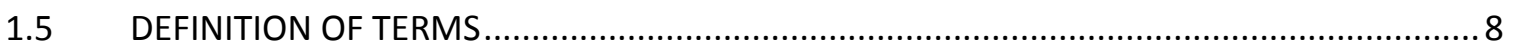

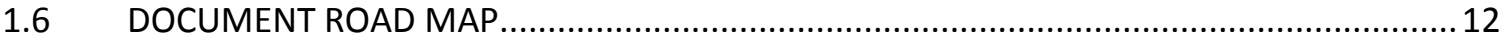

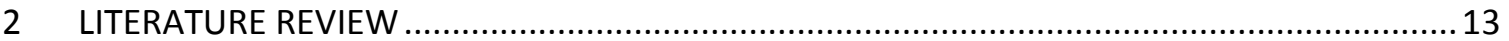

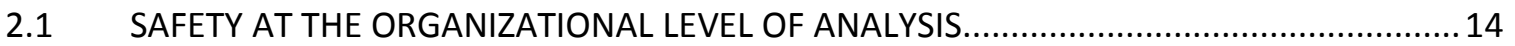

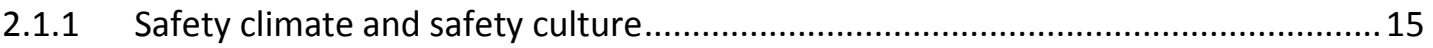

2.1.2 Safety climate in relation to safety performance ................................................. 19

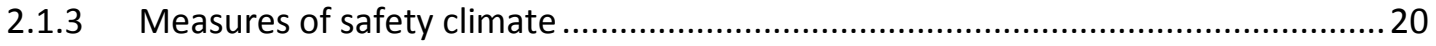

2.2 COWORKER INFLUENCE: SAFETY AT THE GROUP LEVEL OF ANALYSIS.............................22

2.2.1 Early conceptualizations of coworker influence upon safety ..................................23

2.2.2 Modern findings regarding coworker influence .................................................. 25

2.2.3 Mechanisms \& theoretical frameworks of coworker influence in safety studies ... 30

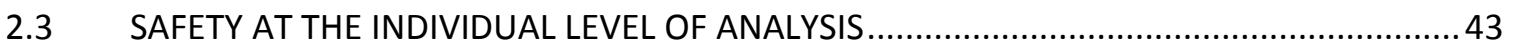

2.3.1 Measuring and defining individual safety performance ........................................ 46

2.3.2 Integrative model of individual safety performance ........................................... 48

2.3.3 Components of safety performance: safety compliance and safety participation.. 50

2.3.4 Determinants of safety: safety knowledge and motivation ..................................52

2.3.5 Antecedents of safety performance - distal situation-related factors.....................53

2.3.6 Antecedents of safety performance - distal person-related factors.......................57

2.3.7 Summary of integrative model of individual safety performance ..........................65

2.3.8 Cognitive mechanisms in the study of individual safety ......................................67

$2.4 \quad$ SAFETY RESEARCH WITHIN INDUSTRIAL SECTORS ..................................................... 75

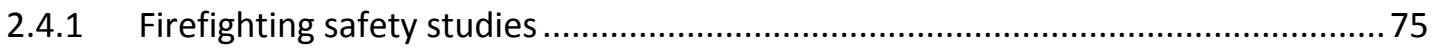

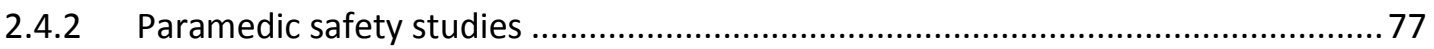

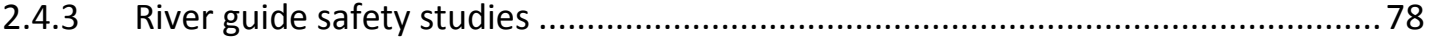

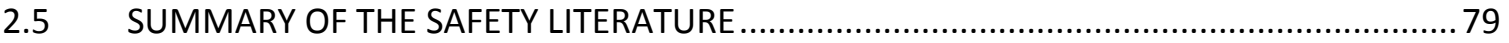


2.5.1 Reviewed safety variables not considered for further study ............................... 82

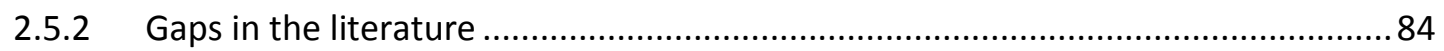

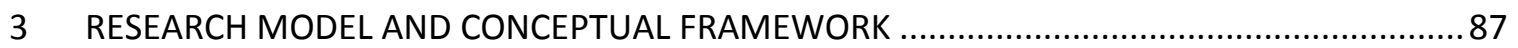

3.1 THEORETICAL CONNECTIONS AND GUIDING PROPOSITIONS …......................................89

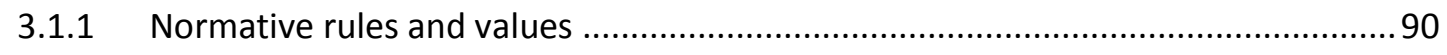

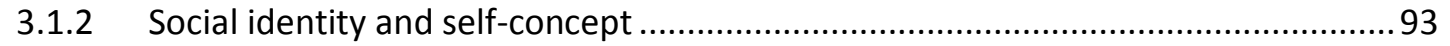

3.1.3 Internalizing coworker and group values........................................................... 95

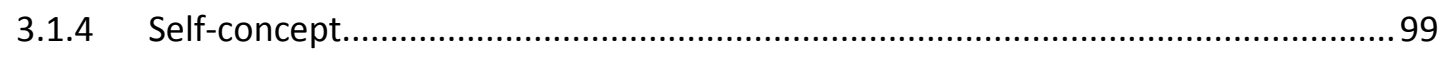

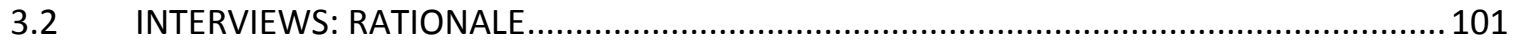

INTERVIEWS: USE OF METHODOLOGY IN LITERATURE ................................................. 103

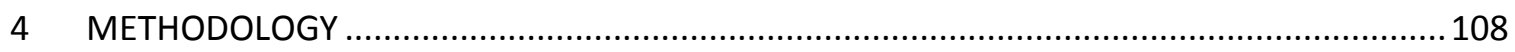

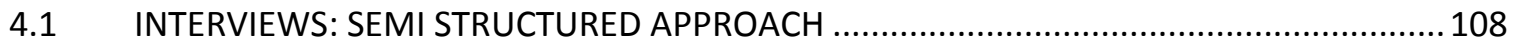

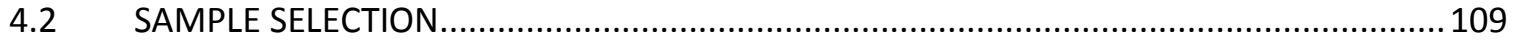

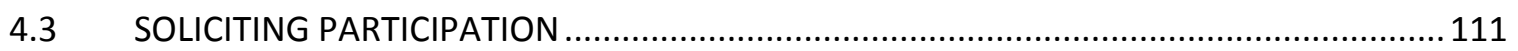

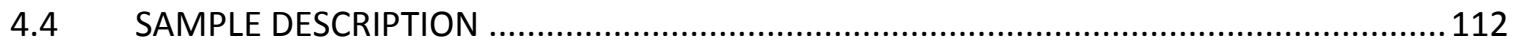

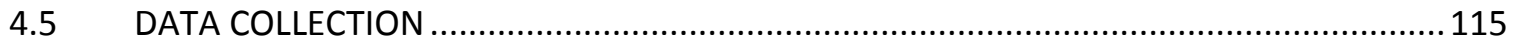

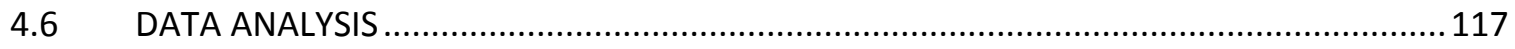

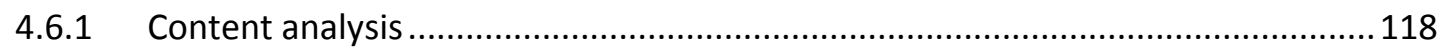

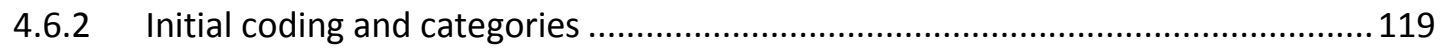

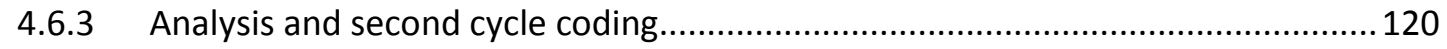

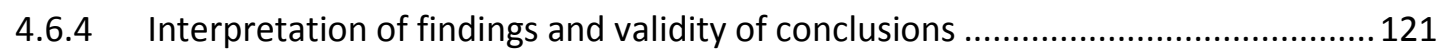

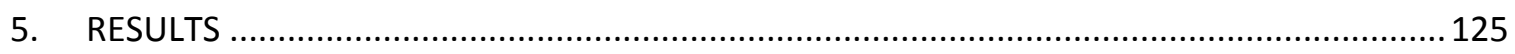

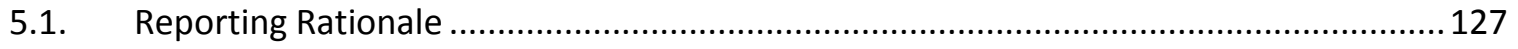

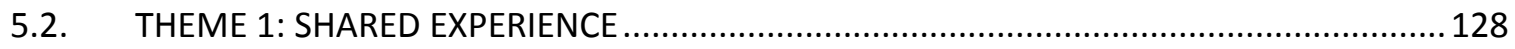

5.2.1. Observation of the workplaces with regards to safety ........................................ 129

5.2.2. Observations of social interaction as shared experience ..................................... 135

5.2.3. Shared experience as foundation for coworker influence...................................... 141

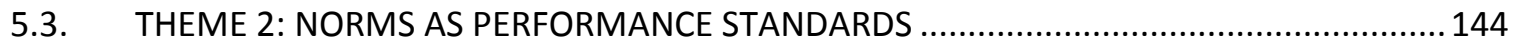

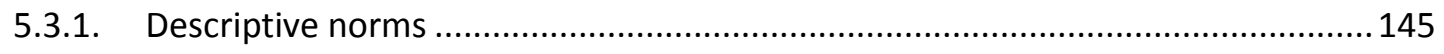

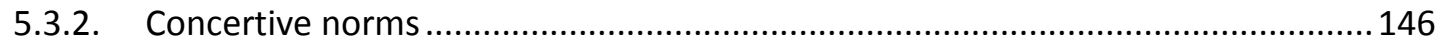

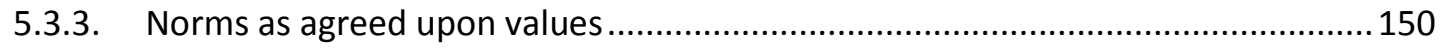

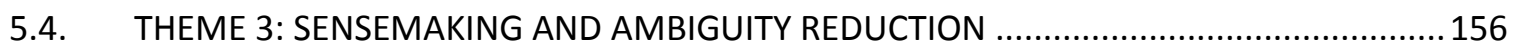




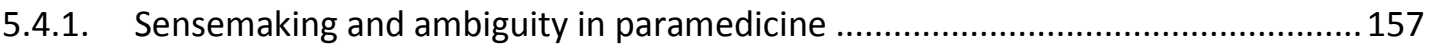

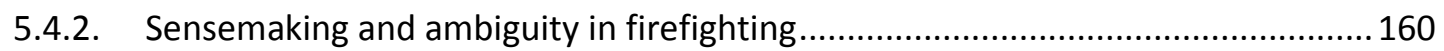

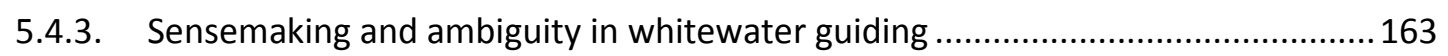

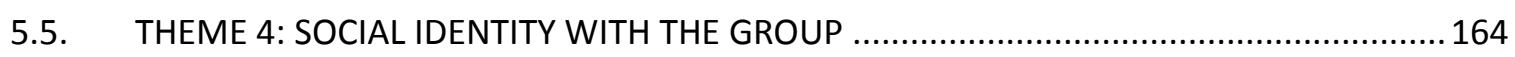

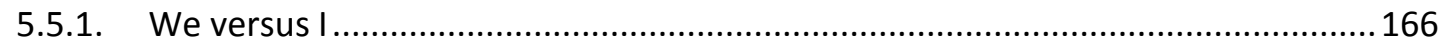

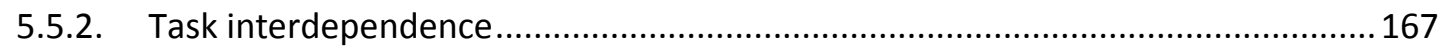

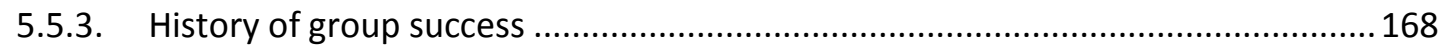

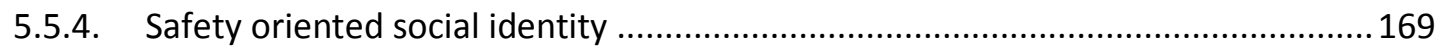

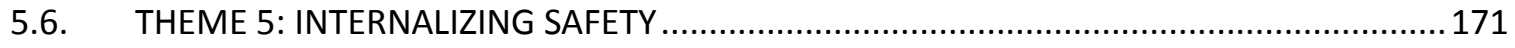

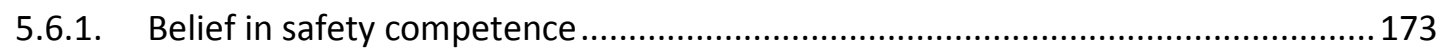

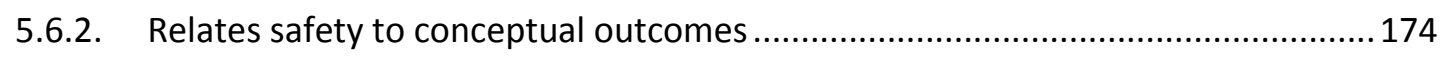

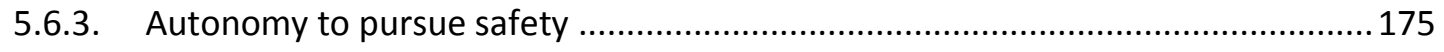

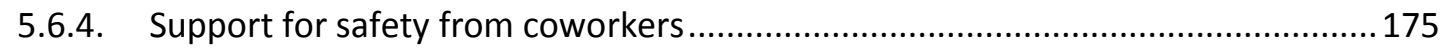

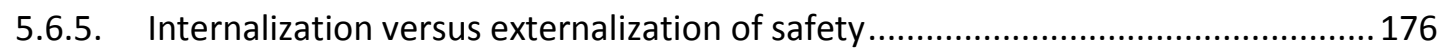

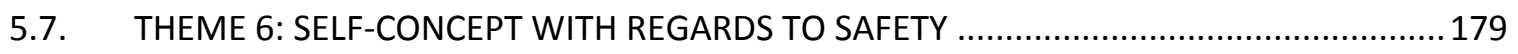

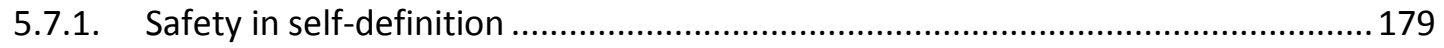

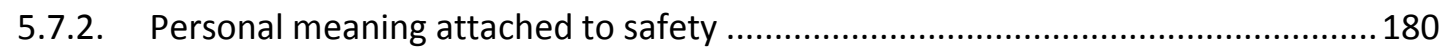

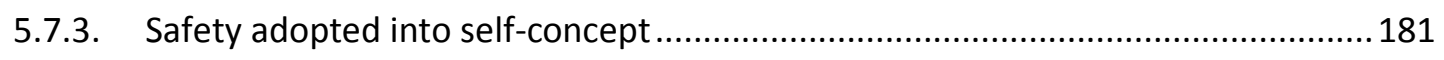

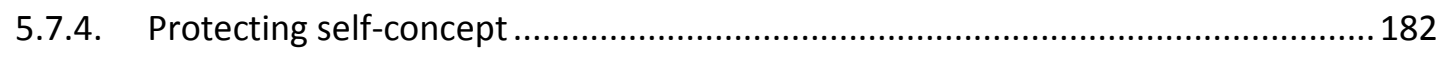

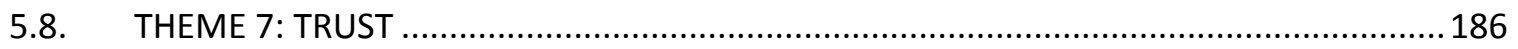

5.8.1. Trust reduces uncertainty with regards to safety outcomes ................................186

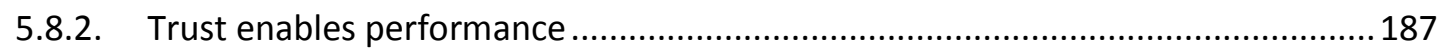

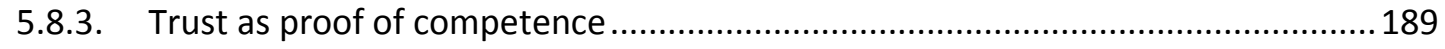

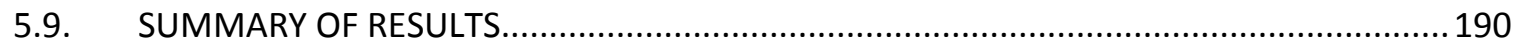

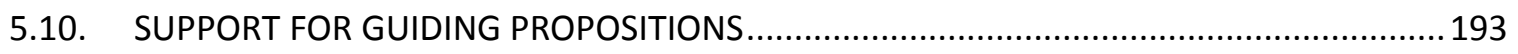

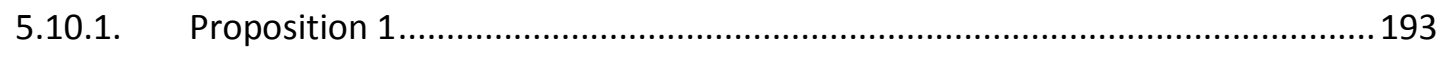

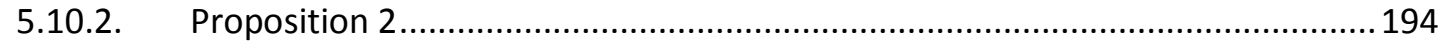

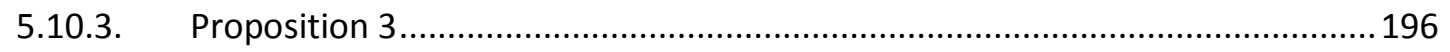

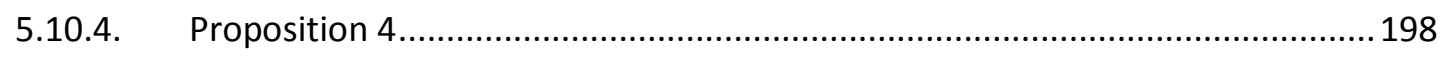

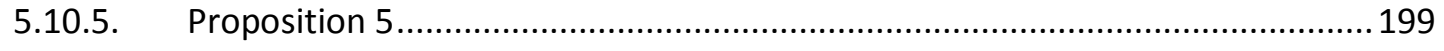

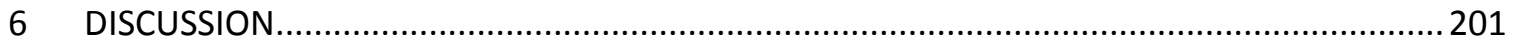

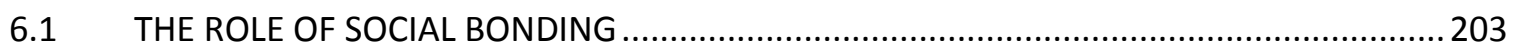




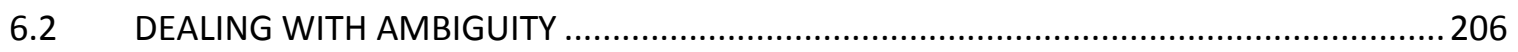

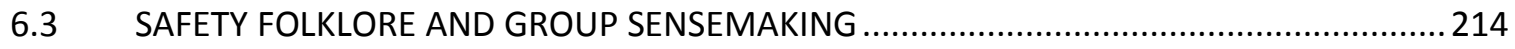

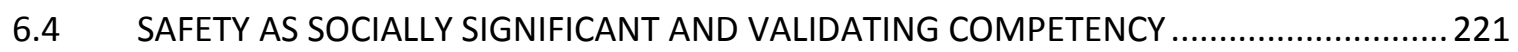

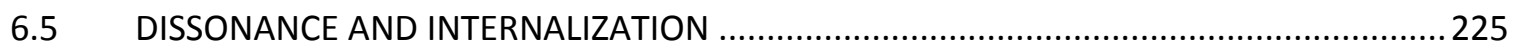

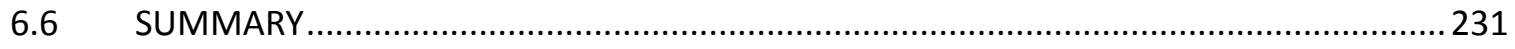

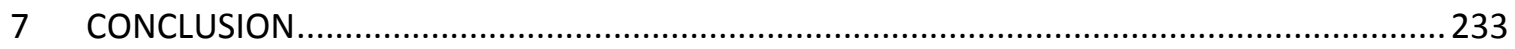

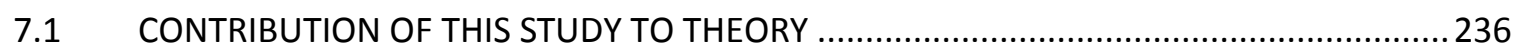

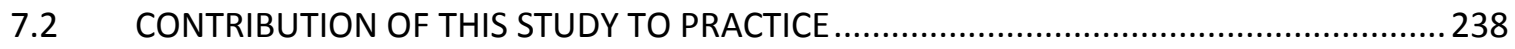

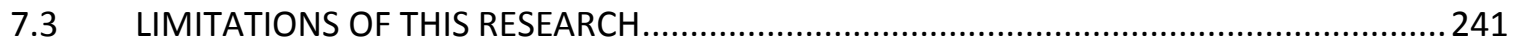

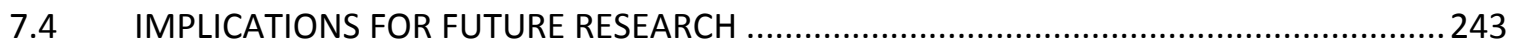

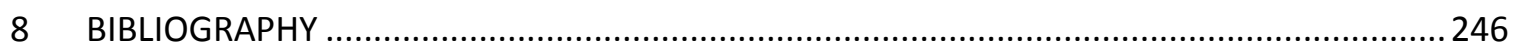

\section{LIST OF TABLES}

Table 1: Demographic distribution of sample ............................................................ 113

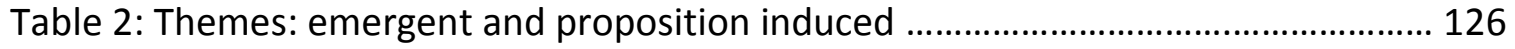

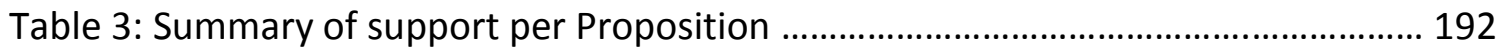

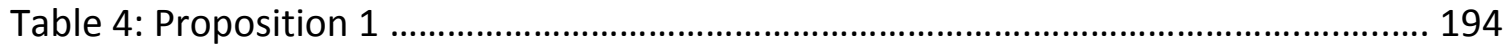

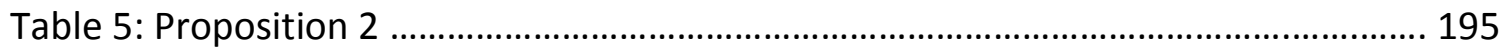

Table 5b: Proportion of individuals who have high safety social identity ..................... 195

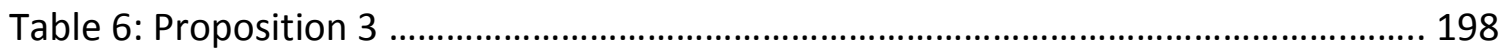

Table 6b: Evidence of Internalization versus Externalization of Safety .......................... 198

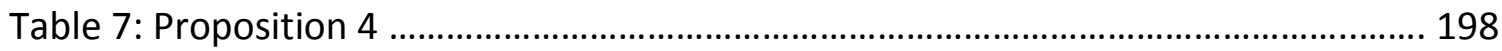

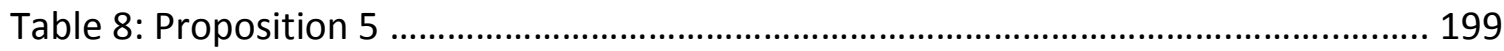

\section{LIST OF FIGURES}

Figure 1: Zohar and Luria (2005) basic group influence model ........................................ 24

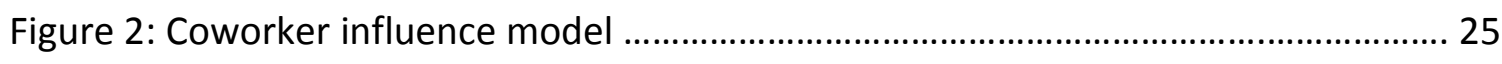

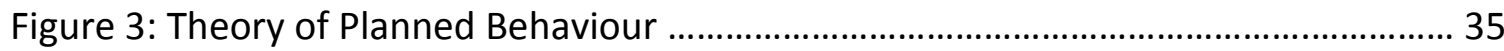


Figure 4: Andriessen's (1978) influence chain upon safety motivation ............................ 45

Figure 5: Integrative model of individual safety performance ......................................... 50

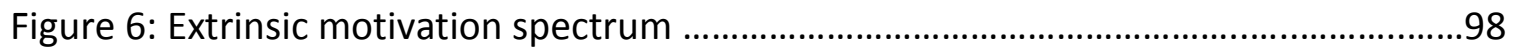

\section{LIST OF APPENDICES}

APPENDIX 1: Urban fire service Email invitation ................................................. 26767

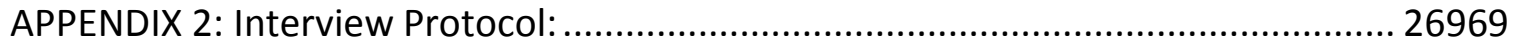

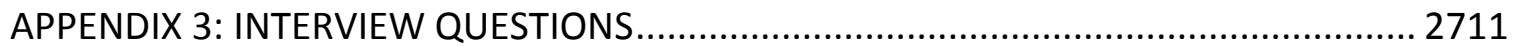

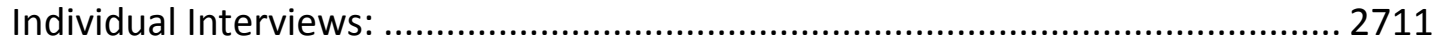

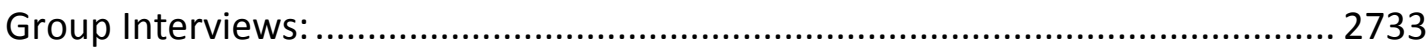

APPENDIX 4: First Cycle and Second Cycle Coding Categories .................................. 27676 


\section{INTRODUCTION}

What is the influence of coworkers upon the individual's internalization of safety? This is the research question guiding this study. Despite it being recognized as one of the most pervasive determinants of individual behaviour, social influence upon individual safety motivation has largely been overlooked by safety research. What began as an exploration of motivation theory applied to individual safety behaviour, safety studies quickly jumped to discussion and research surrounding organizational factors influencing safety, identified as safety culture and safety climate. The majority of this work quantitatively links organization level variables to measures such as accident rates. Little attention has been paid to the motivational aspect of safety, and the role that coworkers play in influencing individual safety intentions.

Qualitative research methodology was utilized to explore coworker influence upon individual internalization of safety. The qualitative approach allowed for the nature of the relationship between the individual and coworker influence to emerge. This research

utilized 55 individual and six group interviews, which were guided by a series of theoretically informed propositions regarding coworker influence. The unit of study was at the individual level of analysis. Information gleaned at the individual level informed the group level investigation of coworker influence.

The sampling frame for this research utilized three sectors within safety critical or 'process safety' industries. Paramedics, firefighters, and whitewater raft guides all share a core 
task of producing safety. Examining three separate sectors allowed for variation in perspective and triangulation of findings.

\subsection{RESEARCH PROBLEM}

Research in the safety field regarding coworker influence on individual safety is only just emerging. Intuitively, there seems to be face validity to the idea that if all of one's peers disregard organizational safety directives, then the individual would understand the 'local' group norm to override the organizational mandate. Likewise, if a group is highly safety oriented, it seems reasonable that an individual group member would understand the performance standard as such. With an emerging awareness of social influence upon safety, a number of authors hypothesize that peers (coworkers) may have a greater influence upon individual safety than does the organization or supervisor. Work by Chiaburu and Harrison (2008) on coworkers in general and by Brondino, Silva and Pasini (2012) and Fugas, Melia and Silva (2011) on safety in particular has concluded as much. However, beyond a discussion of norms, a mechanism for this influence is yet to be proposed.

This research was exploratory in nature, and looked for mechanisms of influence in an asyet unstudied relationship between coworkers' safety orientation and how the individual internalizes safety beliefs.

This research utilized the internalization of safety beliefs at the individual level to examine the social constructs of norms and social influence at the individual level of analysis. Based upon a review of the literature several propositions guided this research. These 
propositions formed the phenomenon under study: how coworkers influence an individual's internalization of safety. This research pursued the question: What is the influence of coworkers upon the individual internalization of safety?

\subsection{IMPORTANCE OF THIS STUDY}

This study is important in that it fills an obvious gap in safety research. By pursuing a research question that asks what the influence is of coworkers upon the individual's internalization of safety, this research contributes an unexamined perspective upon both the study of safety climate and upon the study of safety behaviour.

As outlined in the literature review that follows, research on the social aspect of safety behaviour falls within a multi-level safety climate theory or model. Until recently, social influence on safety (a group level variable) has been restricted to a consideration of only leadership or supervisory influence. The operationalization of social influence within safety climate has been limited. This research is placing the coworker in the context of existing safety climate theory, but is operationalizing the group variable differently - as coworker influence upon the individual.

This research also adds complexity to the field of safety studies by including peer interaction and the internalization of safety values or priorities. Peer interaction has previously not been considered a part of individual safety motivation, nor has the internalization of safety, and these novel perspectives offer significantly more sophistication than the prevailing organizational climate paradigm. As a result, this 
investigation of avenues of coworker influence may identify levers or mechanisms as yet unaddressed in the emerging (larger) coworker influence literature.

Lastly, this research introduces to safety studies as-yet underutilized social theories as a means of examining coworker influence and internalizing safety. In particular, selfdetermination theory and social identification theory are applied to group and individual level safety behaviour for the first time.

\subsection{THEORETICAL FOUNDATION OF THIS STUDY}

This research ultimately explored group influences upon individual safety; consequently falling within the field of work motivation. Work motivation examines the forces that influence the "form, direction, intensity and duration" of individual behaviour (Pinder, 2008: 11). More specifically, under study was how form, direction, intensity and duration of safety related behaviours are influenced by coworkers. This motivation approach to safety studies is a less-common sub-stream within the larger safety field, a field of study typically focussed on engineering and system safety or focussed at the organizational level of analysis. From a motivation perspective, Vroom's (1964) expectancy theory, elaborations upon social cognitive theory (Bandura, 1977) and the theory of planned behaviour (Ajzen, 2001) provided the theoretical foundation upon which this study was built.

Expectancy theory is considered one of the anchor theories of motivation, and "has become a standard in motivation, as reflected by its incorporation as a general framework for a wide variety of research" (Ambrose \& Kulik, 1999: 240). Vroom's (1964) is a cognitive 
process model attempting to explain how decisions are made to achieve an end. Expectancy theory predicts one's effort towards a task based upon a function or probability that one's effort will be successful (expectancy and instrumentality), and the importance of the intended outcome to the individual (valence) (Vroom, 1964). Expectancy is ubiquitous in safety studies (yet rarely specified), as Ford and Tetrick (2008) point to a number of models of personal safety that rely upon a value judgement of relative risk to relative reward, in which valence and expectancy are assumed. Andriessen (1978) was the first to introduce expectancy theory to safety studies, opening a new stream of research now known as safety motivation. Andriessen's (1978) conceptualization of expectancy theory essentially set the safety behaviour research agenda that is still being pursued today, and informs the basic assumptions guiding this research. (Expectancy theory relative to safety is examined in detail in the literature review to follow). While the research agenda in this paper did not explicitly address expectancy theory, aspects of expectancy theory's valence played a fundamental role in the research.

Social cognitive theory (Bandura, 1977) and the theory of planned behaviour (Ajzen, 2001) each occupy anchor positions within psychosocial research, especially within the emerging study of social influence. Both of these theories introduce the normative pressure of social settings, a mechanism that played a pivotal role in this research. Modern elaborations upon these theories are examined below and brought to consideration regarding safety behaviour - in some cases for a first time in this field of study. Social identity theory (Tajfel, 1974; Haslam, 2004), social-information processing 
theory (Salancik \& Pfeffer, 1978) and self-determination theory (Gagne \& Deci, 2005) are related models that are used here to explain a possible means by which coworkers influence individual internalization of safety.

Lastly, this research used the above motivation theories to address a gap in existing safety climate theory. This study operationalized safety climate's group level variable to consider coworker influence, rather than the existing supervisory paradigm. This gap is explained in detail below.

\subsection{VARIABLES UNDER STUDY AND RESEARCH APPROACH}

A social or coworker influence perspective on safety motivation can cover some of the typical shortcomings identified with motivation theory in general. Shamir (1991) argued general motivation theories fall short due to their individualistic bias, based on the "rational maximizer of personal utility" (Shamir, 1991: 406), that focuses on predicting discrete behaviours. This creates a limited view of motivation - one based on selfadministered rewards or expected outcomes - and omits values or moral obligations as a potential impelling force (Shamir, 1991).

Social influence can be defined as the process "whereby individuals' cognitions, attitudes, emotions, and/or behaviours are modified as a function of exposure to (information from) one or more other individuals" (Van Kleef, Van Doorn, Heerdink, \& Koning, 2011: 116). Social motivation implies behaviour is, or can be, intended to satisfy the basic need to belong - one of the important 'core motives' of social motivation (Fiske, 2003). This need 
is present in both classic and modern needs theories, but is typically absent from safety studies.

Social influence research has pursued themes of attitude change (more often referred to as persuasion), compliance with requests, obedience to authorities, and conformity in groups (Cialdini \& Goldstein, 2004; Van Kleef, Van Doorn, Heerdink, \& Koning, 2011), all relevant to safety performance. In all cases, social influence has been found to be "subtle, indirect, and outside of awareness" (Cialdini \& Goldstein, 2004: 591).

Social influence acts via norms. At its simplest form, people behave in line with normative expectations (Manning, 2011). Manning (2011) lists the breadth of work that has proven this point: norms influence individual behaviour regarding recycling, exercise, public transportation, and health choices, for example. As discussed in the literature review, norms have proven to be an important yet largely unspecified influence upon safety behaviour (Johnson \& Hall, 2005; Fogarty \& Shaw, 2010; Zohar, 2000; Fugas, Melia, \& Silva, 2011). Given this history, and the conclusion that coworkers are found to have a unique effect upon individual values and attitudes (Chiaburu \& Harrison, 2008), any new explanation of coworker influence will involve coworker or work group level norms.

This study introduces a social influence (coworkers) upon the individual (internalizing safety) level of analysis. Norms are by their very nature a social construct - they are formed and continue to be informed by those in the social setting (Cialdini \& Goldstein, 2004). This extends up towards safety culture and safety climate as well - both are social constructs. 
By testing theoretical propositions with qualitative interviews, this research method can offer rich insight into social relationships and mechanisms of influence (Pratt, 2009), and potentially add some explanatory perspective to this emerging stream of research within safety studies. Methodology choice and rationale is discussed in detail in following sections.

\subsection{DEFINITION OF TERMS}

Academic literature discussing safety relies upon a variety of safety specific constructs and variables. As an aid to the reader, this safety terminology will be initially defined in order to differentiate the inter-related terminology. Detailed examination of the various constructs and variables will be considered in the literature review that follows.

Safety in everyday language typically means free from harm. In an industrial context, safety is viewed differently, as it is at the same time considered an organizational outcome and an individual input (Christian, Bradley, Wallace, \& Burke, 2009). For example, accident-free project completion, or the number of injury free workdays would consider safety as an outcome measure of a production process. As an outcome, safety measures non-events; safety is realized when nothing negative happens ${ }^{1}$. This makes safety as an outcome measure a difficult concept to operationalize (detailed in the Literature review that follows). The default, then, is often to measure non-safety, or negative events such as injuries and accidents (Reason, 1998).

\footnotetext{
${ }^{1}$ One author calls safety management the 'art of measuring nothing' (Lofquist, 2010)
} 
Conversely, safety can also be considered to be certain practices, procedures or behaviours that contribute to accident prevention - in this case safety is an input. Using protective equipment, following protocols, and looking out for coworkers' well-being are individual actions that contribute to safety (Christian, Bradley, Wallace, \& Burke, 2009). This research deals exclusively with safety as an input, and focuses particularly on the behaviours that contribute to accident or injury prevention, termed safety performance. Put another way, safety performance is defined as the extent to which an individual performs behaviours that increase the safety of the individual, the client, the patient or the public at large (Ford \& Tetrick, 2008). Safety performance, therefore, is an aggregate of discrete behaviours or actions ${ }^{2}$. Like job performance, safety performance has first order measures: an individual can be observed and assessed on his or her safety performance. Safety performance is placed within the larger field of job performance, and is considered a single aspect of job performance (Ford \& Tetrick, 2008). For the purposes of this study, safety performance is considered at the individual level of analysis rather than at an organizational level.

Safety behaviour is the individuals' discrete and specific actions regarding safety on a moment by moment basis (Neal \& Griffin, 2006). These safety behaviours become the input towards safety, and are determined by a combination of knowledge, abilities and motivation (Andriessen, 1978; Neal \& Griffin, 2004). Within the context of safety

\footnotetext{
${ }^{2}$ Some authors do not differentiate between safety performance and safety behaviour, instead using safety behaviour as the globally descriptive term (i.e. Neal and Griffin (2006)).
} 
behaviours, motivation implies both intentions behind and 'energy towards' safety tasks. Safety literature has segmented this construct from the greater motivation field by calling it safety motivation.

Safety motivation is defined as "the individual's willingness to exert effort to enact safety behaviours and the valence associated with those behaviours" (Neal \& Griffin, 2006: 947).

Safety values or safety beliefs inform the 'valence' in motivation, as do safety attitudes inform the willingness to exert effort towards safety. This research is examining how group level values or beliefs are internalized by the individual, recognizing values as an important contributor to motivation and the intentions behind enacted behaviours (Hitlin \& Piliavin, 2004).

Internalization or internalizing safety is defined as the individual "taking in values, attitudes, or regulatory structures, such that the external regulation of a behaviour is transformed into an internal regulation and thus no longer requires the presence of an external contingency" (Gagne \& Deci, 2005: 334). For example, a driver may wear a seatbelt while driving a car to avoid getting a ticket from the police (external regulation). Conversely, a driver can internalize the safety value of wearing a seatbelt so that the act becomes meaningful to the individual (to protect one's self) and becomes internally regulated, regardless of police presence. What's more, one who has internalized safety may also make decisions when it is safer to not wear a seatbelt, even if that means breaking a law. Internalizing safety implies a level of ownership in behavioural outcomes and a conceptualized understanding of cause and effect, regardless of external 
contingencies. Internalizing safety includes adopting beliefs, the value of, and practices contributing to safety.

Organizational constructs regarding safety set the stage for the group level constructs considered in this study. Safety culture is enduring organizational values and beliefs regarding safety, while safety climate reflects the enactment of these values with safety related policies and procedures (Guldenmund, 2000). Organizational safety culture is viewed as more encompassing than safety climate (Choudry, Fang, \& Mohamed, 2007). Safety climate as a concept is intended to capture the priority an organization places upon safety, or to point to the presence of a safety culture. While safety climate deals with formal structures and processes and safety culture deals with enduring values in an organization, safety norms represent informal and social aspects of influence by creating unwritten rules around expected or acceptable safety-oriented behaviour (Fugas, Melia, \& Silva, 2011). Safety norms play an important role in the propositions put foward in this study, as the normative influence of group-generated safety norms is a possible mechanism to influence individual recognition of coworker values. Coworker influences upon internalizing safety is the focus of this study.

The terminology, variables and constructs introduced here will be examined in detail in the sections that follow. There is considerable variation in the literature regarding the precision (or lack thereof) and use of safety terminology; for the sake of consistency, cited authors' ambiguous use of terminology will be converted (where necessary) to the terminology use as adopted in this paper. 


\subsection{DOCUMENT ROAD MAP}

What follows is a complete overview of this research project examining the influence of coworkers upon the individual internalization of safety. A review of the literature briefly examines the organizational level of analysis as context and background, and continues with a complete examination of the emerging group level coworker influence research, as this work plays the most direct role in setting the stage to examine this study's research question. From there, the broad range of variables under study when considering individual safety performance are reviewed, and placed into a comprehensive model of individual safety performance. Lastly, relevant industry sector-specific findings are summarized. This wide-ranging literature review will identify gaps in the current safety literature and set the stage for a conceptual framework and series of propositions that guided this research.

The research model and conceptual framework is outlined, with emphasis on proposed theoretical links to explain coworker influence upon the individual's internalization of safety. Methodology of this qualitative study is then outlined in detail, followed by the results and theoretical discussion. Lastly, the reader will find a summary of this research project's contribution and limitations in its scope. 


\section{LITERATURE REVIEW}

Social influence by peers upon individual safety priorities has been absent from the safety literature. What has tentatively emerged from related papers is an understanding of the role that coworkers may play in individual behaviour (in general) and safety (in particular).

Human behaviour plays a role in most workplace accidents (Ford \& Tetrick, 2008). Some of these human factors are engineered away by modifying materials or redesigning processes (Sanders \& McCormick, 1987). Even with careful engineering, workers still need knowledge and skills, and a separate set of attitudes and values that will motivate safe behaviour (Andriessen, 1978).

Andriessen's 1978 research on safety behaviour at a construction site is considered the first to examine the role of safety motivation (Andriessen, 1978). However, in looking for influences upon individual safety behaviour, industrial research quickly migrated to an organizational level of analysis (Clarke, 2006). The lion's share of the past three decade's safety literature has and is still pursuing organization level studies of safety climate and safety culture. This organization level research is briefly overviewed as context and to set the stage for specific examination of safety literature at the group and individual levels of analysis.

Within the organization level safety culture and safety climate fields, there is a small amount of work on group level culture and group level climate, work that introduces social influence into safety studies. Within the group level, research on safety is almost 
exclusively operationalized around supervisory leadership relative to individual safety performance. There is very little written or understood regarding peer, coworker, or social norms relative to individual motivation and the adoption of safety beliefs. This limited (but emerging) work is examined in detail as it is the avenue through which this research makes its contribution.

At the individual level of analysis, safety research is largely unorganized with multiple disconnected avenues of inquiry. Some of these avenues are direct applications of greater social-psychology streams, while others are safety specific and detached from greater theory. The safety field is still oriented around antecedents and contributors towards safety as opposed to mechanisms and processes by which individuals act. These various interpretations of individual level safety are reviewed below, as are the determinants and antecedents of individual safety performance.

This research investigates coworkers influence upon individual internalization of safety. After setting the stage with an overview of organizational safety literature, the group and individual level safety literature are reviewed, as well as the relevant social and theoretical models that informed this study's research question.

\subsection{SAFETY AT THE ORGANIZATIONAL LEVEL OF ANALYSIS}

The vast majority of safety research is conducted at the industry wide or organizational level of analysis. For this reason, findings from the organization level become important in providing context and setting the stage for any group or individual level of study. Safety 
culture and safety climate are examined below, with particular attention paid to safety climate as it is within this model that this research makes its contribution.

\subsubsection{Safety climate and safety culture}

In looking for influences upon individual safety behaviour, research quickly migrated to an organizational level of analysis (Clarke, 2006), where the majority of safety research is still focussed. Safety climate emerged as an organizational level construct consisting of individual level perceptions (Ford \& Tetrick, 2008), although it has since been revised as a multi-level construct including leadership and group levels of analysis (Fugas, Silva, \& Meliá, 2012; Brondino, Silva, \& Pasini, 2012). Climate, as defined by safety researchers, is rather limited in scope (when compared to organizational or psychological climate), and includes factors such as training programs, management safety attitude, and compliance with statutory regulations (Zohar, 1980). Zohar (1980) is credited as having published the first quantitative study on safety climate, something he defines as the summary of perceptions that employees share about the safety of their work environment. Safety climate has since been shown to significantly correlate to safety compliance, safety participation $^{3}$, accident/injury rates, medical errors, and safety citizenship behaviour (Clarke, 2006; Ford \& Tetrick, 2008). Safety climate as a concept is intended to capture the priority an organization places on safety (Zohar, 1980) - in other words, point to the presence of a safety culture.

\footnotetext{
${ }^{3}$ Safety compliance includes following rules and protocols; safety participation includes elective actions that improve workplace safety.
} 
Some years later, safety culture gained prominence in safety studies, although detached from the simultaneously emerging organizational culture movement in the business field (Guldenmund, 2000). Safety culture became a larger construct than climate, encompassing the enduring values around safety that an organization possesses or tries to establish (Guldenmund, 2010). A significant amount of safety related papers debate the theoretical distinction between safety culture and safety climate (Guldenmund, 2000; Mearns \& Flin, 1999; Ostrom, Wilhelmsen, \& Kaplan, 1993; Zhang, 2002; Hale, 2000), and almost every modern safety culture paper at least acknowledges this debate or distinction in its introduction or literature review. Safety culture authors, in particular, go to great pains to segregate the constructs, while others use the terms interchangeably, and many safety climate papers do not mention culture at all, such as Zohar's (2010) line of climate work.

Safety climate pre-dated safety culture by 15 years, and had an established body of work by the time "culture" emerged in safety language (Guldenmund, 2010). Safety climate is an almost exclusively quantitative endeavour, relying upon large sample surveys to identify mediating or moderating factors within an organization's structure that affect safety performance. Zohar's (1980) original Safety Climate Questionnaire forms the basis for much of what is being studied today (Zohar \& Luria, 2005; Brondino, Silva, \& Pasini, 2012). Conversely, safety culture has proven problematic from an operationalization standpoint, with authors modifying climate surveys or creating a variety of alternative tools, the construct validity of which is debated (Choudry, Fang, \& Mohamed, 2007; Guldenmund, 2010; Glendon \& Stanton, 2000). A wide variety of dimensions are 
incorporated into safety culture studies, with little consensus on what constitutes the boundaries of the safety culture construct (Guldenmund, 2010).

Consensus has emerged upon differentiating safety culture and safety climate by level of operationalization: climate exists at the supervisor and shop floor level (policies and procedures) while culture resides at the top of the organization (with values and beliefs) (Guldenmund, 2000). From this perspective, culture is more encompassing than climate (Choudry, Fang, \& Mohamed, 2007) and safety climate is an expression of an organization's safety culture. In addition, safety climate has been positioned as a 'snapshot' of aggregated individual perceptions at a single point in time, while safety culture attempts to articulate a more enduring values-based construct (Lopez de Castro, Gracia, Peiro, Pietrantoni, \& Hernandez, 2013; Mearns \& Flin, 1999; Denison, 1996). For this reason, inferring culture from climate only captures perception of policies and procedures, as opposed to actual behaviours and beliefs over time (Choudry, Fang, \& Mohamed, 2007). Glendon and Stanton (2000) claim safety culture and climate are best differentiated by methodology: if a psychometric scale is the sole research tool, then climate is being studied, whereas triangulated methods best approach culture. Despite this, many studies of organizational culture rely soley upon employee perception (Allaire \& Firsirotu, 1984).

Glick, as an authoritative voice in organizational climate research, concludes "the minor substantive differences between culture and climate may prove to be more apparent than real" (Glick, 1985: 612). This may be the same for safety studies, as the two are often 
considered simultaneously (either intentionally or not). Despite some writers emphasizing the difference between the two and the need to keep them separate (Choudry, Fang, \& Mohamed, 2007; Lopez de Castro, Gracia, Peiro, Pietrantoni, \& Hernandez, 2013), culture cannot be separated from its climate-based structure and processes $^{4}$ (Guldenmund, 2010). Safety culture, it appears, relies upon safety climate to influence individual behaviour (Choudry, Fang, \& Mohamed, 2007) and in this way, these organizational constructs set the stage for the study of group and individual safety behaviour. Silbey (2009) critiques "Culture as the ultimate, intermediate, or proximate cause often leaves unspecified the particular mechanism that shapes the safe or unsafe outcomes" (Silbey, 2009: 351). Guldenmund (2000) continues that safety culture models are deficient in that they "do not embody a causal chain but rather specify some broad categories of interest and tentative relationships between those... at best they are about the content of safety climate [as opposed to safety culture]" (Guldenmund, 2000: 243). Ultimately, safety requires both an appropriate culture and an appropriate climate (Reason, 1998: 294). While this research study is investigating group influence upon individual level internalization of safety values or priorities, safety culture and safety climate are recognized as both antecedent to individual performance and as context in which social interaction between workers takes place.

\footnotetext{
${ }^{4}$ Antonsen (2009) speaks for a small number of critical theorists and proposes that the term safety culture is not required. In his view, organizational culture combined with safety practices is sufficient to capture what safety culture as a construct is attempting to explain.
} 


\subsubsection{Safety climate in relation to safety performance}

As an antecedent to individual level safety performance, safety climate has been directly linked to safety outcomes; for this reason, climate became the focal construct of study rather than culture (Zohar \& Luria, 2004; Zohar \& Luria, 2005). Clarke's (2006) metaanalysis of 28 safety climate studies found consistent positive correlations between safety climate and safety performance, including compliance, participation, and injury rates. Christian and colleagues (2009) meta-analysis of 90 studies found the same, and observed that safety climate predicted $45 \%$ of the variance in safety motivation, and safety motivation in turn predicted $55 \%$ of the variance in safety knowledge. Both Clarke (2006) and Christian and colleagues (2009) found safety climate to influence safety participation more than safety compliance. Meta-analysis by Nahrgang and colleagues (2011) found safety climate to correlate to job engagement and burn-out. These same authors found evidence of safety compliance as a form of employee engagement. That these metaanalyses found a negative correlation between safety climate and safety criteria (of between -.22 and -.42 ) points to the "robust" predictive validity of safety climate on safety performance. The founding author of this field concludes that "safety climate offers robust prediction of objective and subjective safety criteria across industries and countries" (Zohar, 2010: 1517). This statement is perhaps overly ambitious, as correlation is not causation. Similarly, it is worth noting that although Clarke's (2006) meta-analysis found safety climate is a meaningful predictor of safety performance, it only found a weak relationship to actual safety outcomes (accidents). The original safety climate model incorporated a 'downstream' causal effect, however it is emerging that there is a 
feedback loop or 'upstream' effect upon climate. There is indication that safety outcomes influence safety climate more than safety climate influences outcomes (Beus et al., 2010), and that observations of coworkers' safety performance influences future safety participation, compliance, and motivation (Stride et al., 2013). Clarke (2000) also found that safety climate's impact upon safety performance is not consistent across occupational settings noting "a degree of situational specificity" to safety climate and the likely presence of moderators (Clarke, 2006: 322).

Several variables mediate safety climate, with leadership gaining the most attention (reviewed below as a separate antecedent to individual safety performance). It is here where the study of organizational safety climate is relevant to this present research project; safety climate's operationalization of group level variables has been limited to supervisory leadership (Zohar \& Luria, 2005). An obvious group level climate variable of the coworker has been overlooked, and has only recently been recognized as a gap in the safety climate construct (Fugas, Silva, \& Meliá, 2012; Brondino, Silva, \& Pasini, 2012; Fogarty \& Shaw, 2010; Meliá, Mearns, Silva, \& Lima, 2008). The newly emergent coworker climate stream of research is finding that coworkers are more influential than either supervisory or organizational climate variables.

\subsubsection{Measures of safety climate}

Most of what is known about individual safety performance comes from survey scales developed for organizational safety climate research. Safety motivation, knowledge, participation and compliance findings typically stem from these instruments. Zohar 
(1980) developed the first facet-specific safety climate measure (based on Schneider's 1975 organizational climate measure), which he grouped into the following seven dimensions: (a) perceived management attitudes towards safety, (b) perceived effects of safe conduct on promotion, (c) perceived effects of safe conduct on social status, (d) perceived organizational status of safety officer, (e) perceived importance and effectiveness of safety training, (f) perceived risk level at the workplace, and (g) perceived effectiveness of enforcement versus guidance in promoting safety. Of note is that this scale focussed only on perceptions, and did not ask nor measure actual safety behaviour. These perceptions were measured against injury or accident rates (which occur at asymptomatic levels and have skewed distributions, as noted in the review of the individual level of analysis research below). Zohar's (1980) original hypothesis also indicated that within an organization, the safety climate would be perceived homogenously - a hypothesis that was supported in his original study.

In 2000, Zohar developed a revised Group Safety Climate Scale to test for group level differences in climate. Zohar's (2000) interpretation of group level differences relied upon an assumption that supervisor practice would differentiate groups (and that the groups themselves were homogenous). This scale has been adopted for use by others (Neal \& Griffin, 2006; Zohar \& Luria, 2004) in assessing safety climate as a multi-level construct, but for the most part research has been limited to supervisory or leadership influence. This limited operationalization of group level variables regarding safety climate presents the opportunity that this research pursues, which is to examine the coworker influence upon the individual, rather than supervisory or organizational influences. 
Zohar's (1980) safety climate work and scale of measures wholly dominates the safety culture paradigm as it is understood today. The only minor exception is work by Burke and colleagues (2002), in creating their little used four factor aggregated model of safety performance. These authors created an alternative scale that recently has been picked up and modified to consider coworker influence at the group level (Fugas, Melia, \& Silva, 2011; Fugas, Silva, \& Meliá, 2012). This work will be discussed in sections that follow.

\subsection{COWORKER INFLUENCE: SAFETY AT THE GROUP LEVEL OF ANALYSIS}

Contrary to what many engineers would argue, safety is a social system that relies upon people for effective execution (Cooper, 2000). However, the omission of the psychosocial aspects of safety went unnoticed for some time. Despite being introduced at an early stage (Andriessen, 1978), there has been little attention paid to the social aspects of safety and of coworkers in particular. At the group level of analysis, safety climate usually refers to the role of the supervisor (Zohar, 2000; Zohar \& Luria, 2004; Wallace \& Chen, 2006; Luria, 2008), based upon the logic of proximal safety referents and sensemaking (Zohar \& Luria, 2004). As opposed to organizational level values or policies, supervisors' direct interaction with workers and ability to interpret priorities makes them a more proximal reference on safety expectations and behaviour. Using the same logic, other authors have argued that there is a greater proximal role of the coworker, as the majority of one's day is spent in their presence, unlike that of a supervisor or manager (Tucker et al., 2008; Chiaburu \& Harrison, 2008; Lingard, Cooke, \& Blismas, 2011). From the interactionist perspective, coworkers are likely the most credible referent group for 
individuals, so their "safety beliefs, habits, and behavior as a measure of descriptive norms, are likely to play [an] important role in workplace safety" (Jiang, Yuc, Li, \& Li, 2010: 1469). This has only recently become an agenda item in safety studies.

Safety is a social construction, as is climate and culture (Guldenmund, 2010; Turner \& Gray, 2009). Through this lens, safety is therefore "situated, negotiated, generated, and transplanted in the historical, socio-material, and cultural contexts in which interaction occurs" (Turner \& Gray, 2009: 1260). Guldenmund (2010) argues for organizational safety culture being comprised of many sub-cultures, direct evidence of which has been found in a study of construction sites (Gherardi \& Nicolini, 2002). Each of these sub-cultures reflected work group values - values generated and shared by clusters of coworkers.

\subsubsection{Early conceptualizations of coworker influence upon safety}

Andriessen's (1978) early work on safety motivation discussed primarily organizational and management influences, however he also considered coworker influence. His hypotheses were informed by early group behaviour research, which led him to deduce that "the degree of group cohesion (does the group co-operate well), the group norm (do they regard safety as important) and social pressure (do they watch each other to see whether they live up to group norms)" would positively influence safety motivation of the individual (Andriessen, 1978: 371). His findings confirmed this, specifically that the "reactions of the regular [work] partner appeared to be most important, those of the other colleagues less important, and the reactions of the supervisor the least important" (Andriessen, 1978: 369). Andriessen concluded that a cohesive group works more safely, 
because "a group atmosphere promotes the development of a positive safety norm so that members are supported in their working safely by the approval of their partner and other colleagues" (Andriessen, 1978: 374). While most aspects of Andriessen's work carried on into subsequent research, this coworker aspect did not.

The eventual inclusion of a group level variable into the safety climate construct was directed towards leadership or supervisory influence (Figure 1) (Zohar \& Luria, 2005). But it was Melia (1998) who proposed (in Spanish) the first psychosocial model of workrelated accidents that placed coworkers as a variable of influence. His model relied upon a mechanism similar to Bandura's (1977) social learning theory, that supervisor and coworker responses would inform the individual's behaviour. He positioned workplace agents in a causal chain: the organization's safety climate influences the manager, the manager influences coworkers; the coworkers in concert with the climate and manager influence on the individual. The individual's behaviour influences accident rates (Figure 2) (Meliá, 1998; Meliá, Mearns, Silva, \& Lima, 2008). Interestingly, in only two of Meliá and colleagues (2008) four samples did the supervisor response predict worker safety response.

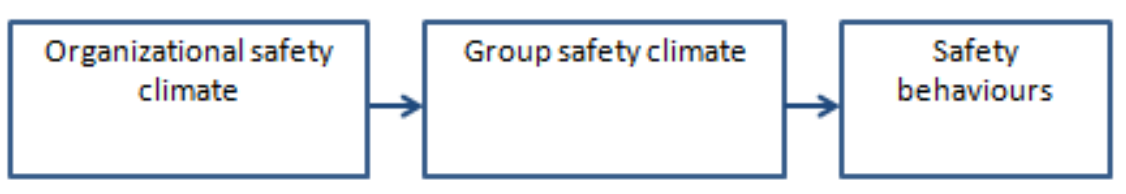

Figure 1: Zohar and Luria (2005) basic group influence model 


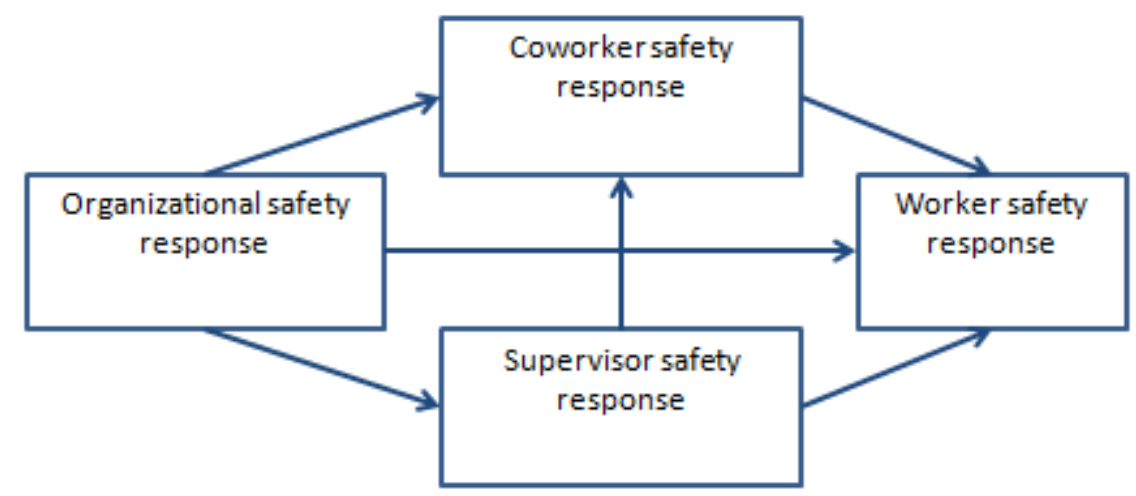

Figure 2: Coworker influence model (Meliá, Mearns, Silva, \& Lima, 2008)

\subsubsection{Modern findings regarding coworker influence}

The gap in the literature regarding peer influence has come to the attention of a range of researchers. Safety research has not systematically included coworkers (Brondino, Silva, \& Pasini, 2012), despite them being an "important yet often overlooked social influence upon safety" (Tucker et al., 2008: 322). For example, Christian and colleagues (2009) in their comprehensive meta-analysis on workplace safety antecedents make no mention of peer or coworker influence.

A number of researchers have reconsidered the relative influence of the supervisor versus coworkers. Brondino and colleagues (2012) found that the supervisor has only a minor role in influencing individual safety behaviour when compared to organizational safety culture and coworker influence. The supervisor did not mediate the relationship between the organization and the coworkers, contrary to their hypothesis (Brondino, Silva, \& Pasini, 2012). Fugas, Melia, and Silva (2011) found that supervisors and coworkers 
influence individual safety differently and uniquely, and offer a mechanism by which this influence is applied. In their study, supervisor safety practices (i.e. role modeling) did not impact individual safety behaviours (reviewed in detail below).

Yagil and Luria (2010) found social support from coworkers has a positive effect upon safety behaviour. Safety behaviour is highest when both social support and climate are high. When climate is low, social support increases the level of safety behaviour suggesting that high-quality social relationships with coworkers could buffer the effects of low-level safety climate (Yagil \& Luria, 2010). Tucker and colleagues (2008) found that perceived coworker support fully mediated the relationship between the likelihood of an individual voicing safety concerns and safety climate. This supportive environment not only has a positive effect upon safety but with other work outcomes such as burnout and job engagement (Nahrgang, Morgeson, \& Hofmann, 2011). Coworker support for safety has been identified by several authors as an important dimension of safety climate (Törner, 2011; Zohar, 2000; Meliá, Mearns, Silva, \& Lima, 2008; Neal \& Griffin, 2006).

Coworkers have been found to have direct impact upon an individual's safety values, attitude, and motivation. Jiang and colleagues (2010) found individual safety behaviour is directly influenced by observation of coworkers and perceptions of their safety beliefs. Westaby and Lowe (2005) found coworker risk taking was a greater influence upon individual risk taking than was supervisor influence. Zhou and colleagues (2008) concluded coworkers influence safety attitudes more than does individual education or work experience. From this study, $46 \%$ of construction workers surveyed said coworkers 
had a positive influence upon their safety, and $4.7 \%$ said they had a negative influence. In a training context, Chiaburu (2010) found coworker support had the greatest impact on training transfer to the workplace and skill maintenance, over supervisory or organizational support $(r=.49$ compared to $r=.24$ and .18$)$. Interestingly, safety participation is found to be more affected by coworkers than is individual safety compliance - a finding in line with safety climate's influence on participation over compliance (Neal \& Griffin, 2006; Fugas, Melia, \& Silva, 2011). Why compliance (following protocols) has less social influence than safety participation (elective actions that improve workplace safety), has yet to be explained in the literature.

While qualitatively pursuing factors underlying individual level work safety behaviour, Mullen (2004) found social influence was a pervasive motivator. Socialization into the job position, desire to maintain a tough image or an appearance of competence, or avoiding harassment were all identified as important in directing safety behaviour. Mullen (2004) concluded that:

Individuals in this study believed that in order to maintain their desired image, they had to comply with subjective norms in the workplace, which resulted in performing unsafe behavior [...] It was found that even under conditions in which safety was highly valued and rewarded by management, workers chose to work unsafely just to portray a particular image that was deemed important and desirable. Thus, individuals who have the need to maintain a desirable image will be more likely to comply with the perceived subjective norms regarding safety in the organization. (Mullen, 2004: 282)

While a small, fragmented group of authors were conceptualizing peer influence upon worker performance across a wide range of sectors, a 2008 meta-analysis by Chiaburu 
and Harrison on coworker effects upon perceptions, attitudes, organizational citizenship behaviour (OCB) and performance opened the door and enabled very specific coworker influence models to emerge. Importantly, Chiaburu and Harrison (2008) provided the first empirical support indicating that coworker actions predict perceptions, attitudes, and behaviour outcomes of their colleagues even when accounting for the influence of the direct leaders, based on an examination of 161 data sets. Coworker support was more predictive than leader support for job involvement $(p=.33$ coworker support and $p=.06$ for leader support) and was important for withdrawal behaviours (e.g., $p=.22$ vs. $p=.04$ for effort reduction, and $p=.08$ vs. $p=.01$ for absenteeism, coworker, and leader support respectively) (Chiaburu \& Harrison, 2008: 1089).

Drawing on work that ranged from the 1950's to 2007, Chiaburu and Harrison (2008) assembled a rationale to explain their findings. The authors differentiated between vertical and horizontal influence upon the individual. Vertical influence is based upon authority and hierarchy (upon which the predominant safety climate paradigm is based), while horizontal influence is based upon more frequent and less restricted social interactions as coworkers share the same status and work side by side. Coworkers offer information, support, and role modeling, all of which shape beliefs and attitudes regarding valence of priorities and behaviour. Chiaburu and Harrison (2008) argue that these frequent, informal interactions serve several important purposes: to reduce role ambiguity (uncertainty regarding behaviour expectations and unpredictability of behavioural outcomes); to reduce role conflict (the balance of conflicting priorities, such 
as between production and safety); and how to internalize and externalize "core elements" of one's job (Chiaburu \& Harrison, 2008: 1084).

The authors present a model whereby coworker support (or its opposite, antagonism) is mediated by joint relationships of social intensity of the work situation, support content, and antagonism severity. These in turn influence employee perceptions, attitudes, participation, and performance. No mechanism by which these relationships act is proposed, "commanding" further exploration (Chiaburu \& Harrison, 2008: 1095).

Brondino, Silva, and Pasin (2012) picked up Chiaburu and Harrison's (2008) work and tested a multi-level safety climate model that included the overlooked coworker element. By surveying almost 1000 workers in five manufacturing plants, they found that coworker safety climate had a stronger mediating role with safety behaviour than did supervisor safety climate. Supervisor safety climate only partially mediated the relationship between organizational safety and coworker safety climate (Brondino, Silva, \& Pasini, 2012). Multilevel structural equation modelling pointed to coworker safety climate as reducing the effect of the supervisor safety climate - a finding uncovering substantially more complexity in the formation of safety climate than the predominant hierarchical safety climate paradigms. This finding is in line with Chiaburu and Harrison (2008), and Brondino and colleagues (2012) echo Chiaburu and Harrison (2008) calling for more investigation into the lateral relationships among coworkers and the potentially reciprocal relations with supervisory climate (i.e. additive, interactive, compensatory). 
Research has recognized the relevance of coworkers, and their influence upon individual behaviour is becoming clear (Brondino, Silva, \& Pasini, 2012). This, combined with the important role group perceptions play in forming safety climate (and safety climate's positive relationship to safety outcomes), means that coworker influence deserves further examination. However, the missing link is the mechanism or processes underlying these direct relationships (Chiaburu \& Harrison, 2008). Some of these proposed mechanisms are considered below in the key works informing this emerging stream of research.

\subsubsection{Mechanisms \& theoretical frameworks of coworker influence in safety studies}

Social psychology identifies a wide range of means by which social influence plays a role in directing individual behaviour. Only a select few of these appear in safety studies. Implicit in a psychosocial model of influence is that interactions between the leader and the worker, or between the coworker and the individual, have the ability to modify one's behaviour (Barsade, 2002; Brondino, Silva, \& Pasini, 2012). While the organization, leadership and work group provide the context in which one acts, safety research points to the norms that these agents generate that are influential. The influence of norms may be via values or attitudes, which are socially influenced (Parks \& Guay, 2009), or by some other means. Further, the limited representation of social influence theories in safety research is introduced below. 


\subsubsection{Norms}

People behave in line with normative expectations (Manning, 2011) and norms have a "contextual influence" upon individual attitudes (Fugas, Melia, \& Silva, 2011: 67). Manning (2011) lists the breadth of work that has proven this point: norms influence individual behaviour regarding recycling, exercise, public transportation, and health choices, for example. Organizational behaviour carries similar lines of reasoning via organizational culture research (Schein, 1985). Norms are implied in climate and safety culture (Hofmann \& Stetzer, 1996), but they have not been addressed directly until recently (Fugas, Melia, \& Silva, 2011). Climate deals with formal structures and processes or culture with enduring values in an organization, while norms represent informal and social aspects of influence (Ehrhart \& Naumann, 2004). Vaughn (1996) discusses the NASA Challenger explosion and shows how norms regarding safety come to be constructed by work groups. Safety violations can come to be viewed as 'normal'. By normalizing deviance, a norm of tolerance is generated (Vaughn, 1996).

Workers recognize a difference between coworker observed behaviour and an understanding of how one ought to behave (Lingard, Cooke, \& Blismas, 2011), known as injunctive and descriptive norms. Injunctive norms are perceptions of what relevant others want one to do with regards to behaviour. Descriptive norms are perceptions of what relevant others actually do with regards to behaviour (what ought to be done versus what is done) (Fugas, Melia, \& Silva, 2011). Differentiation between the two types of norms has been empirically supported both in safety literature and in general (Manning, 2011; Fugas, Melia, \& Silva, 2011; Lingard, Cooke, \& Blismas, 2011). In organizational 
studies, injunctive norms were thought to trump descriptive norms, and descriptive norms are often referred to only when injunctive norms are weak (Cialdini \& Goldstein, 2004). Recent safety specific studies find the opposite: coworker descriptive norms had the most influence upon individual safety participation (Fugas, Melia, \& Silva, 2011; Fogarty \& Shaw, 2010). This is in line with the work of Chiaburu and Harrison (2008), presented earlier. What coworkers actually do may carry more influence than what coworkers say or believe.

Norms require a referent group (Cialdini \& Goldstein, 2004). Normative influence has typically been envisioned as originating at the supervisory level, and only recently at the peer level of influence (Stride et al., 2013; Garcia-Herraro, Mariscal, Gutierrez, \& TocaOtero, 2013; Fugas, Melia, \& Silva, 2011). Interestingly, Westaby and Lowe (2005) measured coworker 'informational influence' (i.e. descriptive norms) rather than considering that coworkers may have an injunctive influence. These same authors investigated supervisor injunctive norms. Despite mixing descriptive and injunctive norms, coworkers were found to have the greatest influence upon risk taking behaviour at work. This finding reinforces the importance of what coworkers actually do versus what is said or believed.

Fugas and colleagues (2011) explain that social norms from groups that are more powerful or proximal to the individual, and more relevant to the worker, are more likely to result in behaviour change than norms from less relevant groups. The authors suggest that when a number of group members perform certain safety behaviours, and there is a 
shared recognition in the group that these safety behaviours are considered standard, the behaviours become recognized as normative, and safety descriptive norms are established (Fugas, Melia, \& Silva, 2011). From this point, norms can be described by their intensity and crystallization (Jackson, 1975). Intensity is a measure of group pressure towards certain behaviour, and crystallization is the degree to which a group agrees upon this pressure. Crystallization implies a group widely believes a norm to be 'correct' (Fugas, Melia, \& Silva, 2011). Crystallization is one means of explaining findings by Zohar and Fussfeld (1981), who observed workers continued to wear ear plugs several months after an ear plug compliance program was revoked; this despite a high rate of worker turnover and many who had not been exposed to the initial safety program. A new norm may have crystallized that earplugs were now proper and expected.

Fogarty and Shaw (2010) found group norms to explain $42 \%$ of the variation in individual safety attitude and $36 \%$ of individuals' compliance intentions. Norms also predicted $41 \%$ of safety violations (Fogarty \& Shaw, 2010). The authors noted that these values are larger than those typically found in organizational studies. A related study found similar results, with coworker descriptive safety norms mediating the relationship between organizational safety climate and individual safety participation (Fugas, Silva, \& Meliá, 2012). These findings raise the question, is safety behaviour somehow more affected by descriptive norms than other types of workplace performance?

Group social identity can generate powerful group values and norms (Barker, 1993). These norms not only provide direction to the individual, but also reduce ambiguity and 
clarify performance expectations (Hogg \& Terry, 2000). Andriessen (1978) recognized this

in his early work, but only briefly addressed the idea:

Groups that are well coordinated in their work and in which there are few misunderstandings work more safely. This is the case because such a group atmosphere promotes the development of a positive safety norm so that members are supported in their working safely by the approval of their partner and other colleagues. (Andriessen, 1978: 374)

The obvious question that arises when discussing norms in a safety context is: how do they influence individual motivation and behaviour? Cognitive models such as social exchange theory and social learning theory imply norms act via role modeling approved behaviours. The theory of planned behaviour offers a more complex dual process theory that includes subconscious aspects to social influence. These potential mechanisms are examined below.

\subsubsection{Theory of planned behaviour}

There are very few theoretical frameworks brought to the safety field to explain the relationship between safety variables and safety behaviour. The most convincing model introduced to date is the theory of planned behaviour (TPB). This theory (tested and applied to a long line of social psychology research) predicts behavioural intentions are a combination of individual attitude (value judgements towards an action) and perceptions of social pressure (norms), plus perceptions of control of outcomes (Ajzen, 2001). In pursuing attitude research, Ajzen (2001) hypothesized that attitude's weak correlation with individual behaviour was due to intervening factors. These intervening factors were found to be subjective norms and perceived behavioural control (Figure 3). The presence 
of norms in this model makes it as applicable to the study of social influence as it is to individual intention and behaviour.

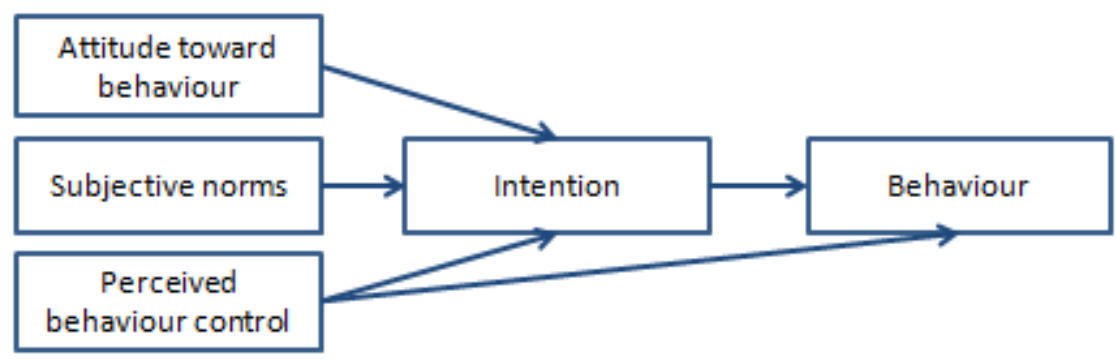

Figure 3: Theory of Planned Behaviour (Ajzen, 1991)

With the introduction of this model, attitudes and perceived behavioural control have gained prominence in the study of social behaviour (Fugas, Silva, \& Meliá, 2012), of which perceived behaviour control was previously unexamined in safety research. Johnson and Hall (2005), in the first study to introduce TPB to safety, found that perceived behaviour control was the strongest predictor of safe lifting behaviour in a warehouse. Norms played a lesser role and attitudes did not register as a predictor of safe behaviour, but did mediate the other two variables (Johnson \& Hall, 2005). In the only safety related complete test of the TPB, Fogarty and Shaw (2010) found that perceived behavioural control, group norms, and personal attitudes combined to explain $50 \%$ of the variance in self-reported safety violations (Fogarty \& Shaw, 2010). In their study, group norms had the largest influence on intentions and on individual attitude. Fogarty and Shaw (2010) also found that individual attitude, norms and perceived behavioural control were all influenced by 'management attitude' (i.e. safety climate and culture). Interestingly, Fogarty and Shaw (2010) measured TPB variables against safety violations, and positioned 
violations as volitional, deliberate actions (i.e. choosing to be unsafe), and therefore based on attitudes and behaviours and applicable to TPB. This rational definition of behaviour ignores the wider slips, lapses and mistakes (Reason, 1997) or the more pervasive subconscious influences upon behaviour (Pinder, 2008).

In a meta-analysis, Manning (2011) found both conscious and subconscious ('nondeliberative') effects of norms within TPB. Norms do not have to be in focus to direct behaviour, at least for socially motivated actions. This makes TPB one of many dual process theories that exist in social psychology. Dual process theories build upon a belief that judgements are mediated by both fast, automatic processes and more deliberate, analytic ones; a differentiation that is now well established (Evans \& Frankish, 2009). Dual process theories have not made any inroads in safety studies, and this aspect of TPB has yet to appear in safety literature.

With regards to social behaviour, Strack and Deustch (2004) conceptualize a widely cited dual process model that involves both a 'reflective' and 'impulsive' evaluation of social expectations. The reflective system is the conscious and cognitive evaluation of social cues, while the impulsive system relies upon associative links and intentions (e.g. needs). These two systems operate independently and yet inform one another, generating eventual understanding or behaviour (Strack \& Deutsch, 2004). Manning (2011) uses this model to account for normative perceptions in TPB. The reflective system responds to norms brought into focus, while the impulsive system integrates norms' influence below the level of conscious recognition. 
Fugas and colleagues (2012) looked at the cognitive and social mediating mechanisms in the TPB with regards to safety compliance and safety participation. The authors broke norms into supervisor and coworker descriptive and injunctive norms, and examined how they influenced individual safety attitudes and perceived behaviour control. Safety participation was mediated by coworker descriptive norms and individual safety attitudes; compliance was fully mediated by supervisor injunctive norms and perceived behavioural control (Fugas, Silva, \& Meliá, 2012). These findings point to the important role social influence plays in all aspects of safety performance. Individual safety performance is related to perceived coworker practices. These authors called for more qualitative studies to further understand the complexities in these relationships.

Ajzen's (2001) original description of TPB explained perceived behavioural control as a combination of control beliefs: internal and external factors that impede performance. These included self-efficacy (internal) and perceived power (external). This opens the door to a potential link to personality studies, where Christian and colleagues (2009) found safety performance was moderately related to locus of control.

\subsubsection{Social exchange theory}

Social exchange theory (SET) is quoted as "among the most influential conceptual paradigms for understanding workplace behaviour" (Cropanzano \& Mitchell, 2005; 874). Social exchange theory is based upon interdependent and contingent interactions that generate perceived obligations (Blau, 1964). These obligations generate norms of reciprocity (Chiaburu \& Harrison, 2008). Where there is a visible commitment to safety in 
an organization, workers are thought to recognize this and reciprocate with safer behaviour (Hofmann \& Morgeson, 1999).

Safety climate relies upon SET extensively to explain how an organizational level construct directly influences the individual (noting that earlier safety climate research overlooked the new found mediating factor of coworkers). An organization or supervisor who cared for one's safety, it is assumed, would generate a reciprocal response of safe behaviour (Zohar, 2000; Hofmann, Morgeson, \& Gerras, 2003; Clarke, 2006). This was often bundled, however, with rewards and expectancy, which has some face validity - an organization that values and rewards safety creates valence regarding safe behaviours. Whether it is the reward or created norm of reciprocity at play is not metered out.

Organizational citizenship behaviour also relies upon social exchange (Chiaburu \& Harrison, 2008), and so SET and reciprocity is brought to safety participation papers (Neal \& Griffin, 2006; Clarke, 2006) as safety participation is seen as a form of OCB. Workers will voluntarily contribute to their coworkers' safety when they see their coworkers contributing as well (Brondino, Silva, \& Pasini, 2012; Tucker et al., 2008). As in the larger organizational studies, SET has been applied to leader member exchange, and Tucker et al. (2008) applied the same to safety supervision. High quality relationships between supervisor and workers saw greater voicing of safety concerns, however coworker support fully mediated the effect (Tucker et al., 2008). These authors introduced Latane's (1981) social impact theory (which proposes that strength, proximity and number of 
people have greater influence upon the individual) to explain the role of the supervisor, but did not extend this to their findings of coworker mediation.

In their review of SET, Cropanzano and Mitchell (2005) discuss the important role norms play in social exchanges. The norm of reciprocity is more important for the norm it creates, or reflects, rather than the reciprocity phenomenon (Cropanzano \& Mitchell, 2005). According to these authors, these norms become 'rules of exhange', informed by organizational values and individual values, attitudes and motivation. Chiaburu and Harrison (2008) suggest that social exchanges between coworkers are more useful for the development of clear beliefs of what is expected of the individual - generating norms greater than just reciprocating a good deed.

\subsubsection{Social learning theory}

Stride and colleagues (2013) hypothesized via social learning theory that by observing others perform unsafe acts in effect makes such acts permissible. Observing coworkers becomes an enabler or barrier to safety compliance and safety participation. Stride and colleagues (2013) found that greater incidence of witnessing unsafe behaviour is correlated with greater perceptions of barriers to safety participation, and witnessing injuries as barriers to safety compliance.

Social learning theory states that people learn by cognitively processing observed action and information (Bandura, 1977). Key drivers of learning include observed consequences of others' experience (vicarious positive or vicarious negative reinforcement), as well as previous direct experience (positive or negative reinforcement) and anticipated 
experience (the promise of positive or negative reinforcement). This theory overlaps with descriptive norms, in that individuals observe each other's behaviour and learn what is considered to be acceptable. Social learning theory relies upon gauging the outcomes that others experience to inform one's own decisions and behaviour. Stride and colleagues (2013) concluded "regardless of organizational prescription, employees may learn safety norms from the unsafe behaviour of their coworkers" (p. 47).

Beus and colleagues (2010) meta-analysis of safety climate studies reinforces social learning theory. The authors concluded that witnessing coworkers' unsafe behaviour or workplace injuries has a greater influence upon safety climate than does the opposite (witnessing safe behaviour). Safety climate studies recognize that these observations erode perceptions of safety and therefore erode safety climate strength (Zohar, 1980; Zohar \& Luria, 2005). The influence of observing unsafe behaviour may help explain the earlier point that descriptive norms tend to override injunctive norms in regards to safety (Fugas, Melia, \& Silva, 2011).

\subsubsection{Other mechanisms of social influence upon safety}

Beyond norms, social exchange and social learning, no other theoretical framework or mechanisms have widespread adoption in the safety literature; however, there are a small handful of alternative approaches that can be found. One angle considers variations on coworker ability to influence role perceptions (and thereby influence behaviour). Chiaburu and Harrison (2008) found that coworkers both reduce role ambiguity and role conflict. The authors write, "coworker influences primarily-perhaps exclusively-affect 
how their colleagues perceive and shape their work roles, and how they form, retain, and access work attitudes. These role perceptions and attitudes, in turn, carry forward to changes in performance," (Chiaburu \& Harrison, 2008: 1086). A study in nuclear power operations found that clarifying role conflict and role ambiguety led to improved safety compliance (Martínez-Córcoles, Gracia, Tomás, \& Peiro, 2014). Climate and culture play a role in reducing role ambiguity. Luria and Yagil (2010) found that permanent employees relied upon organizational and group level referents to clarify safety roles, while temporary workers looked to proximal coworkers for direction.

Some authors refer to individual role clarification as an exercise in sensemaking. When contextual cues are ambiguous, individuals turn to others for social verification (Festinger, 1954; Weick, 2010). Sensemaking is, therefore, an ongoing, socially based interpretative process that constructs interpretations of ambiguous situations (Brown, 2000). Zohar (2010) claims that the shared perceptions that become safety climate are formed through 'symbolic social interaction', where one compares their own perceptions against others', and over time they converge into group shared meaning. Zohar (2010) calls this shared meaning sensemaking.

In addition to coworker influence in sensemaking and role perceptions, cognitive dissonance also appears in safety studies. Used primarily in the safety culture literature, cognitive dissonance is proposed as the means by which cultural values become adopted by individuals in an organization. This theory believes that individuals have a need for consistency, and when there is inconsistency or dissonance the result is psychological 
tension and the individual will act to relieve that tension (Festinger, 1957). Guldenmund (2010) uses cognitive dissonance to explain how individuals are motivated to adopt organizational safety values and align their own values and safety behaviours to reduce dissonance. It would be difficult, Guldenmund (2010) implies, to hold and act upon individual values that differed from organizational culture values. The individual will change their values to align with that of the organization. By this means, organization level safety culture is enacted at the individual level.

Those authors that rely upon cognitive dissonance as a mechanism to explain safety culture tend not to detail the process of individual values adoption, electing to allude to or only use the theory at a superficial level. As used by Guldenmund (2010) cognitive dissonance has much in common with operant theories used in safety studies, as it omits the role of intermediary norms and coworker influence. This contrasts with modern cognitive dissonance theory, which recognizes the influential role of norms in establishing standards of behaviour (Cooper, 2011).

Mullen's (2004) findings, cited earlier, offer an alternative mechanism or motivation for individual safety behaviour. She found behaviours surrounding safety were intended to preserve one's image or self-esteem. This introduces social identity theory (Haslam, 2004), self-concept (Shamir, 1991) and the powerful influence of social norms, none of which Mullen (2004) elaborates upon in that paper. 


\subsubsection{Summary of theoretical frameworks of coworker influence in safety studies}

The direct attention paid to safety norms (in safety literature) has opened the door to understanding the complex social influences upon individual safety. Coworker descriptive norms have proven important - what coworkers do carries more weight than what coworkers say or believe; more important than supervisor or organizational direction. Norms have proven influential with regards to individual safety attitude, and when they work together both norms and attitudes may mediate safety participation.

Cognitive models such as SET and social learning theory imply norms act via role modeling approved behaviours, reinforcing the agency approach to safety behaviour. TPB offers a more complex dual process theory that includes subconscious and social influence, alluding to the 'non-deliberative' aspects of behaviour.

The study of norms recognizes that group social identity can generate powerful group values (Barker, 1993). Where these group values come from is not made clear, nor their relationship to individual values. This allows the question: Are safety norms rooted in individual values, or is there a collective sensemaking process by which consensus emerges on group values? Do these group norms, in turn, change individual values or just influence individual behaviour? These questions reflect gaps in the safety literature and influenced the direction of this research project.

\subsection{SAFETY AT THE INDIVIDUAL LEVEL OF ANALYSIS}

Andriessen's 1978 research on safety behaviour at a construction site is considered the first to study safety motivation at the individual level of analysis (Andriessen, 1978). Prior 
to this, safety research (at the individual level of analysis) was focussed on identifying 'accident proneness' and identifying characteristics that made certain individuals more or less safe than others (a 'correlational approach'), or by testing safety improvement programs (a 'behaviourist approach')(Grindle, Dickinson, \& Boettcher, 2000). Andriessen's paper is largely overlooked today, with authors often citing more recent work that has clear ties back to Andriessen's original article (often unattributed, such as that of Neal and Griffin (2006)). Andriessen's safety paradigms and findings more or less covered the safety research agenda that is still being pursued today. Importantly, Andriessen (1978) anchored his model of safety within work behaviour, reliant upon motivation, knowledge, and abilities. Specifically, "someone will act unsafely when he does not know what a safe way is, and/or when he is not able to do it more safely and/or when he is not motivated to do it safely" (Andriessen, 1978: 364). Separate from knowledge and skills, Andriessen (1978) argues a set of particular attitudes and values are required to motivate safe behaviour. Only a few alternatives to this paradigm have been explored (detailed below).

A second enduring paradigm introduced by Andriessen (1978) is that of a distal/proximal influence chain upon individual safety motivation (Figure 4). Safe work behaviour is most influenced by motivation elements of values, attitudes, knowledge, and skills. These, in turn, are influenced by the separate norms established by coworkers and the work leader. Distal factors of organization, management, group, individual and task characteristics are least influential upon individual behaviour. This model has not significantly been altered by modern findings, and the third level - safety norms - has only been explored recently. 


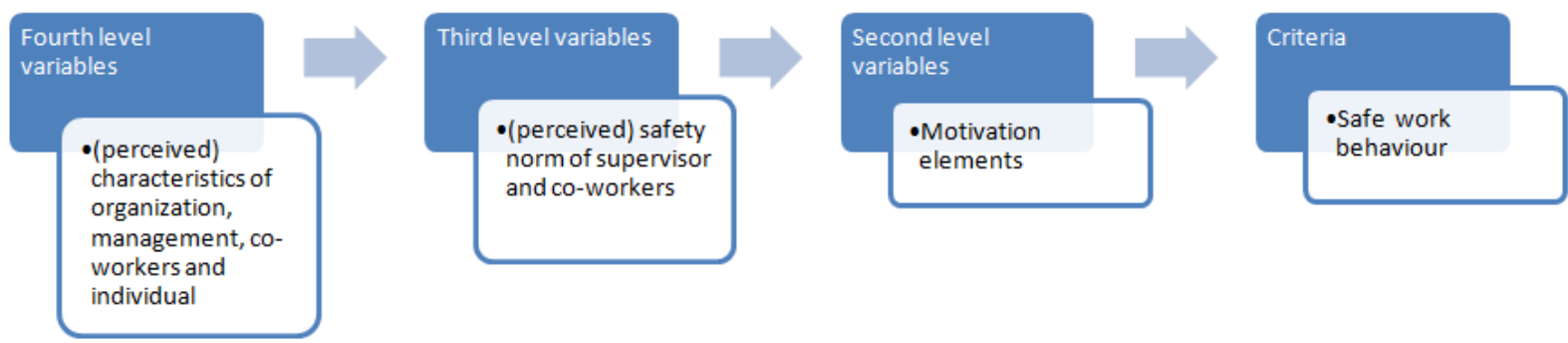

Figure 4: Andriessen's (1978) influence chain upon safety motivation

Andriessen's (1978) findings were based on surveys administered to 207 construction workers. His data showed a differentiation between what has become known as 'safety compliance' and 'safety participation' (Griffin \& Neal, 2000). These, he found, were independent variables. Safety compliance is an individual level construct that Andriessen (1978) called 'carefulness' and refers to following rules and behaviours leading to one avoiding injury. On the other hand, safety participation applies to taking initiative and contributing to improving workplace safety (Andriessen, 1978). The latter has since been aligned with safety citizenship behaviour, and OCB (Hofmann, Morgeson, \& Gerras, 2003). This differentiation between safety compliance and safety participation has become an organizing feature of modern individual level safety motivation research (Clarke, 2006; Neal \& Griffin, 2006).

Additionally, Andriessen (1978) listed further findings: that safety motivation is strongly determined by leadership and the safety standards of the leader; that personality traits do not strongly influence safety motivation; that workers show more safety initiative when the supervisor and colleagues react positively to it; and that safety motivation is also determined by group standards and group cohesion (Andriessen, 1978: 363). Each of 
these findings predated and summarized what are now individual streams of research leadership, individual differences (personality), and group and social influences - the latter of which has only recently gained academic attention. These streams are reviewed in turn, below.

\subsubsection{Measuring and defining individual safety performance}

In their 2009 meta-analysis of safety performance, Christian and colleagues (2009) identify the problematic set of inconsistent definitions and measures of safety. The authors note that safety can be both an organizational outcome (number of injury free workdays) or an individual level input (complying with procedures or identifying deficiencies). This is problematic in that each likely has different relationships with their antecedents.

Safety and safety performance is a difficult concept to theorize, as safety is measured more by an absence than by a presence (Reason, 1998). As an outcome measure, safety is realized when nothing negative happens. Safety outcomes measure non-events, and non-events are invisible since there is no deviation from the expected outcome. The default, then, is often to measure non-safety, or negative events such as injuries and accidents. Many authors caution against the use of negative safety outcomes as a measure of safety behaviour, as negative outcomes occur at an asymptomatic level (Reason, 1997), have a low base rate with skewed distributions (Zohar, 2000), and injuries are "not always a likely outcome of even the riskiest of behaviour" (Ford \& Tetrick, 2008: 1475). 
Invisible safety outcomes are also misleading as they require a "number of dynamic inputs to create stable outcomes" (Reason, 1998: 294). Zohar (2000) argues that these behavioural inputs can be predicted with greater accuracy than can safety outcomes. It is for these reasons that this research project and literature review focuses only upon safety as an input and as behaviour.

To this end, safety performance is considered a distinct construct while at the same time a subset of job performance (Ford \& Tetrick, 2008; Christian, Bradley, Wallace, \& Burke, 2009), aligning with the previously identified definition:

Safety performance is the extent to which an individual performs behaviours that increase the safety of the individual and organization and avoids behaviours that decrease safety of oneself and the organization. (Ford \& Tetrick, 2008: 1473)

These safety behaviours are determined by a combination of knowledge, abilities and motivation (Andriessen, 1978; Neal \& Griffin, 2004). Safety motivation is considered in this paper to be "the individual's willingness to exert effort to enact safety behaviors and the valence associated with those behaviors" (Neal \& Griffin, 2006: 947).

Safety values inform the 'valence' in motivation, and safety attitudes inform the willingness to exert effort towards safety. Meta-analysis has confirmed that safety performance is strongly related to both safety knowledge $(p=.61)$ and safety motivation $(p=.57)$ (Christian, Bradley, Wallace, \& Burke, 2009). Safety knowledge is simply the understanding and knowledge of hazards and safe working procedures. 


\subsubsection{Integrative model of individual safety performance}

Neal and Griffen (2004) proposed a model similar to Andriessen's (1978) and further articulate the link between safety performance antecedents. These authors argue that organizational safety climate and individual personality directly influence safety motivation and knowledge, which in turn directly influence safety performance behaviours, which then directly relate to negative safety outcomes of accidents and injuries (Neal \& Griffin, 2004). In this model, safety climate and personality become antecedents to safety; motivation and knowledge become determinants of safety; and behaviour can be broken into components of safety (Vinodkumara \& Bhasib, 2010). Christian and colleagues (2009) expanded upon Neal and Griffin (2004) with a more thorough model, organizing the antecedents of safety as they appeared in the literature to date (Figure 5). Their meta-analysis of 90 articles on safety performance found this model to hold, and so it will be relied upon to organize this individual level of analysis literature review.

Christian and colleagues (2009) found that safety outcomes were a direct result of safety performance. Safety performance is in turn influenced by safety motivation and safety knowledge. The authors positioned safety motivation and knowledge as 'proximal' individual factors, separate from 'distal' factors. Christian and colleagues' (2009) contribution to the literature was to organize these distal factors into situation-related or person-related categories, putting the very large situation-related factor field (i.e. organization level safety climate) into perspective against individual safety performance. 
Situation-related factors were limited to organizational safety climate and leadership, while person-related factors included personality characteristics and job attitudes.

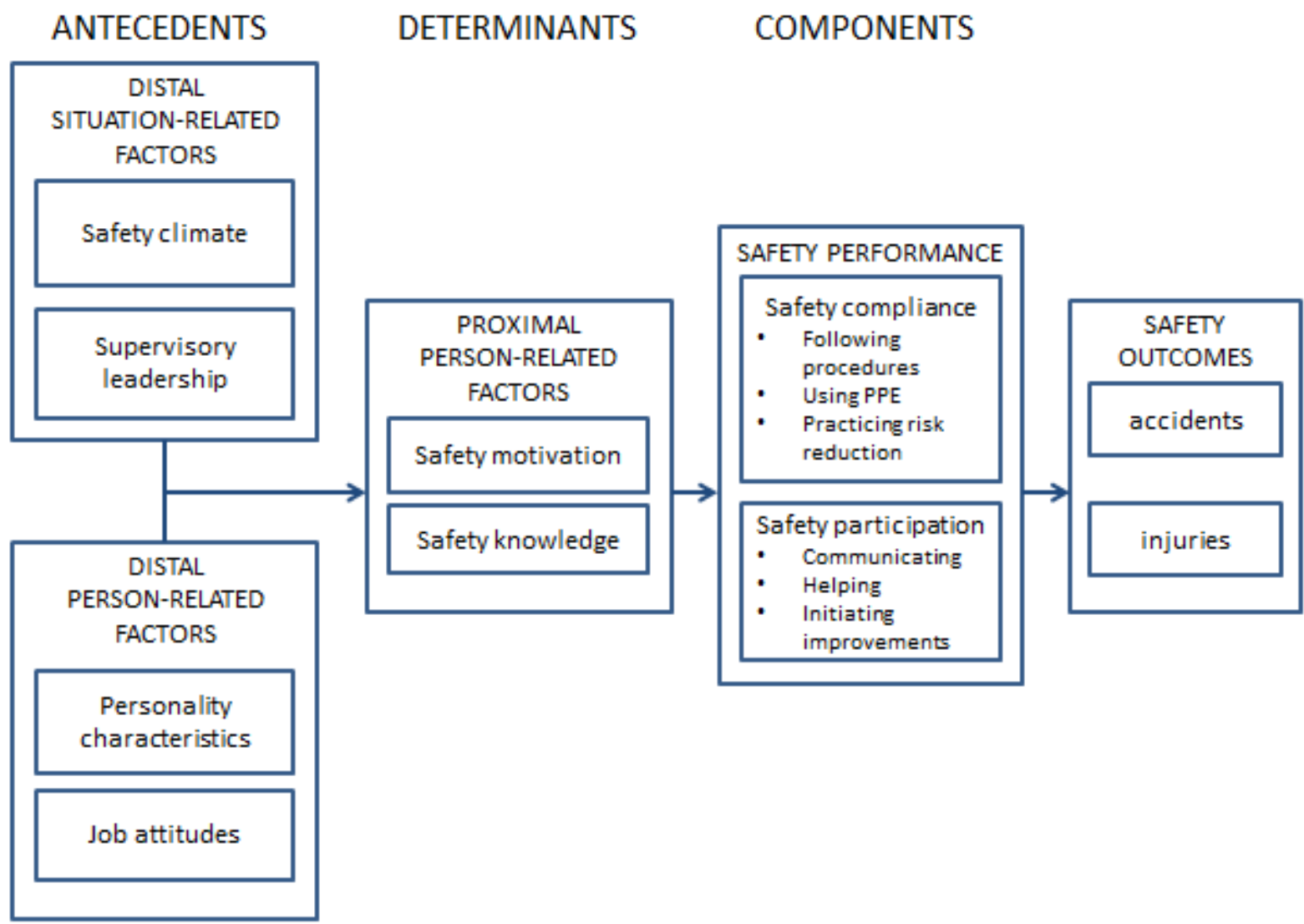

Figure 5: Integrative model of individual safety performance (Christian et al. (2009))

As an overview of these relationships, proximal factors were shown to have a greater relationship with safety than distal factors, and safety performance was strongly related to safety knowledge and safety motivation (mean correlation of 0.61 and 0.57 respectively) (Christian, Bradley, Wallace, \& Burke, 2009).

Findings regarding distal person-related factors indicated safety performance was moderately related to locus of control (mean correlation of 0.35 ) but only weakly related 
to conscientiousness and risk taking (mean correlation of 0.18 and -0.28 ). Locus of control was also stronger in relation to safety participation than to compliance. Job attitudes were weakly related to safety performance (mean correlation of 0.25 ). Distal situation factors were found to have a moderate relationship with safety performance. Safety climate and leadership had mean correlation values of 0.49 and 0.31 respectively.

It is interesting to note that much of these findings were predicted by Andriessen in 1978. This raises the question, what have safety studies accomplished then if they have not traveled far from original findings? Törner (2011) writes “Much research effort has been spent on trying to establish the nature of antecedents of occupational safety, but less safety research has focused on trying to disclose the mechanisms for such influence" ( $p$. 1262). Some of these few tentatively proposed mechanisms are reviewed below and is the purpose of the study at hand.

\subsubsection{Components of safety performance: safety compliance and safety participation}

The differentiation between safety compliance and safety participation has become an organizing feature of modern individual level safety research (Clarke, 2006; Neal \& Griffin, 2006; Ford \& Tetrick, 2008; Vinodkumara \& Bhasib, 2010; Fugas, Melia, \& Silva, 2011; Stride et al., 2013). Safety compliance includes all "core activities" that individuals must execute in order to maintain a base level of workplace safety (Neal \& Griffin, 2006: 947). This would include wearing personal protective equipment (PPE), following procedures, and fulfilling maintenance requirements. Safety participation describes discretionary behaviours that do not directly improve the individual's safety but instead supports or 
develops a culture of workplace safety (Neal \& Griffin, 2006). This would include helping coworkers, devising safer work procedures, discussing safety, or enforcing safety values and norms. Participation behaviours are typically considered elective. Safety compliance and safety participation equate to task and contextual performance from the greater job performance field, although the overlap is not complete enough for them to be considered as the same things (Burke, Sarpy, Tesluk, \& Smith-Crowe, 2002).

Safety participation is found to be more affected by safety climate and by coworkers than is individual safety compliance (Neal \& Griffin, 2006; Fugas, Melia, \& Silva, 2011; Brondino, Silva, \& Pasini, 2012; Clarke, 2006). Why compliance has less social influence has yet to be explained in the literature. Compliance is, however, affected by supervisory oversight more so than safety participation (Fugas, Silva, \& Meliá, 2012), potentially due to the elective nature of safety participation. Proposed mechanisms for these affects and relationships will be discussed in a later section of this literature review.

Safety participation has received much more attention in recent years than has safety compliance, likely due to safety participation's direct link to the overbearing safety climate literature. Perceived organizational support, for example, is considered an important variable that mediates safety participation (Tucker et al., 2008), but is also considered within safety climate scales (Brondino, Silva, \& Pasini, 2012). Safety citizenship behaviours are considered a form of safety participation (Hofmann, Morgeson, \& Gerras, 2003), which in turn are considered part of the larger OCB construct (Ford \& Tetrick, 2008). Hofmann and colleagues (2003) identify 6 safety citizenship behaviours similar to 
general OCB. These include safety related helping, making safety related recommendations, stewardship in caring for the safety and well-being of coworkers, whistle blowing on other staff members who do not follow safety procedures, civic virtue through keeping informed about safety-related policies and procedures, and initiating safety-related change (Hofmann, Morgeson, \& Gerras, 2003).

An alternative model of safety performance exists, but has gained little traction likely due to its similarity with compliance and participation: Burke and colleagues (2002) defined safety performance as "actions or behaviours that individuals exhibit in almost all jobs to promote the health and safety of workers, clients, the public, and the environment" and includes four factors: using personal protective equipment, exercising employee rights and responsibilities (both of which could be considered safety compliance); engaging in work practice to reduce risk, and communicating hazards and accidents (both of which could be considered within safety participation) (p. 432). These authors found the four factors as distinct but correlated, and recommend aggregating them. As components of safety or as contributing to safety motivation, these aggregated factors have not been pursued by researchers.

\subsubsection{Determinants of safety: safety knowledge and motivation}

Neal and Griffin (2004) positioned safety knowledge and safety motivation as determinants of safety compliance and participation. Within the study of safety performance, safety knowledge is considered to be somewhat constant. Andriessen (1978) proposed this (but did not test it) when he assumed that skill and ability to act 
safely were always present, focussing instead upon one's motivation to act. He wrote “...work behaviour will probably be determined more by motivation because prerequisite knowledge and skills needed will usually be present as a result of selection and training" (Andriessen, 1978: 364). Safety motivation, as the individual's propensity to utilize one's knowledge and abilities to safely address the task at hand, typically becomes the active variable. Where safety knowledge is studied, it is typically approached pragmatically to identify better policy or compliance programs, such as mine safety initiatives (Laurence, 2005), or fleet driver training (Mooren, Grzebieta, Williamson, \& Olivier, 2014). Interestingly, training has been proven to have a greater effect upon safety motivation than it does upon safety knowledge (Vinodkumara \& Bhasib, 2010).

The variable of safety motivation is typically found to be more strongly related to safety participation, and concurrently safety knowledge is more related to safety compliance (Griffin \& Neal, 2000; Vinodkumara \& Bhasib, 2010; Neal \& Griffin, 2006), yet at a metaanalytic level, safety knowledge has equivocal results in predicting compliance (Christian, Bradley, Wallace, \& Burke, 2009). The direction of the causal arrow is up for debate in these relationships, as safety participation could conceivably improve safety motivation (Neal \& Griffin, 2006), just as motivation could improve safety knowledge.

\subsubsection{Antecedents of safety performance - distal situation-related factors}

Christian and colleagues (2009) organized the distal antecedents of safety performance into situation-related and person-related factors. Situation-related factors are organizational safety climate and leadership, examined individually below. These 
situation factors become the context and environment in which individuals make decisions or respond to cues and so play a prominent role in safety research.

\subsubsection{Safety climate and safety culture}

Examined earlier in this literature review, safety climate and safety culture provide important context to the study of safety at the group or individual level of analysis, but also form a proven antecedent situation-related factor. The work by Christian and colleagues (2009) did not differentiate between safety culture and safety climate, but its examination at the meta-analysis level dealt primarily with climate factors.

Safety climate is a reflection of the psychological work environment and acts as a frame of reference for individual motivation and behaviour. From this perspective, safety climate becomes a framework for group norms, individual safety priorities and motivation, making it "an important cornerstone of contemporary thought about health and safety" (Fugas, Silva, \& Meliá, 2012: 468). This research project is re-considering the operationalization of safety climate's group level variable by examining the role of the coworker and their influence upon individual safety internalization.

\subsubsection{Leadership}

The role of leadership in regards to individual safety performance shows two influences.

One path emerged from safety climate group level studies which offered fairly linear (single factor) evaluations of leadership, while a second path independently introduced complexity (multi-facet) leadership via organizational leadership findings into the safety field. This latter stream is very active in current years. 
That leaders influence climate is a fundamental climate assumption (Lewin, Lippitt, \& White, 1939). This positive relationship between leadership and safety climate has been proven repeatedly (Hofmann \& Morgeson, 1999; Hofmann, Morgeson, \& Gerras, 2003; Zohar \& Luria, 2004). When Zohar (2000) expanded the study of safety climate to include group level differences, he relied upon leadership and supervision as the differentiating factor between groups. Zohar explained his logic as:

Supervisors execute [organizational] procedures by turning them into predictable, situation-specific action directives... The fact that procedures rarely cover every situation implies a certain level of supervisory discretion in policy implementation... resulting in distinctively different perceptions of instituted procedures versus supervisory practice in individual subunits. (Zohar, 2000: 587588)

The scale used by Zohar (2000) focussed on perceptions of supervisory balance between production and safety, and dealt with rule breaking and openness to suggestion.

These first studies found that the organization level safety climate had a greater influence upon safety outcomes than did the leader or group level (Zohar \& Luria, 2005). In this way, leadership played a mediating effect between organizational climate and individual perceptions. More recent meta-analysis, however, has reversed this finding showing now that group level influences have a greater impact than organization level climate (Christian, Bradley, Wallace, \& Burke, 2009). One effect is by mediating organizational climate, while leadership also has a stand-alone moderating effect (Clarke, 2013). Consistently, leaders are found to have a stronger influence upon safety motivation than upon safety compliance (Christian, Bradley, Wallace, \& Burke, 2009). More recent work has undermined the influence of leadership, finding it secondary to coworker influence. 
These findings were examined in detail in coworker influence/group level of analysis section of this literature review.

Leadership models have been introduced to safety from management studies. Within these models, transformational leadership has received the most attention (Clarke, 2013). These studies find that the quality of relationship with one's supervisor predicts individual level safety behaviour (Hofmann, Morgeson, \& Gerras, 2003), and that laissezfaire leadership has a negative effect on safety, including reduced compliance and participation (Mullen, Kelloway, \& Teed, 2011). Individualized leader/member exchange (LMX) relations and feedback is found to have a direct positive effect on individual safety behaviour (Zohar \& Polachek, 2014), especially safety participation (Sampson, DeArmond, \& Chen, 2014). The supervisory safety schema is found to differ from workers', and is centred on values, communication, and practice versus the workers' schema of procedure and training (Colley \& Neal, 2012).

Transactional leadership is found in safety studies to be distinct from transformational leadership (monitoring, assisting, problem solving versus sharing values and priorities) (Clarke, 2013), and transactional leadership with regards to safety is typically studied in a negative light. Negative transactional leadership (correcting workers) was found to have no impact on safety performance (Luria, 2008), but Clarke (2013) studied positive transactional leadership (monitoring, assisting, problem solving) and found it to more strongly influence safety compliance than transformational leadership did ( $p=.41$ vs. $p=$ 
.31). Safety participation was more influenced by transformational leadership, pointing to the need for both types of leadership behaviour.

The mechanism by which leadership acts is hypothesized to be one of role modeling and sensemaking. Supervisors offer via words and actions an interpretation of often contradictory organizational demands or policies, thereby interpreting cues for workers. Leaders, in effect, act as a safety referent (Zohar \& Luria, 2005). The supervisor's proximal role relative to organizational leadership makes him/her more influential, and this sensemaking and role modeling, Zohar (2000) hypothesizes, creates expectancy and valence relative to safety behaviour.

Leadership relative to safety is a robust and growing field of modern study. While the results outlined here are representative of the field, a complete review of leadership relative to individual level safety is outside the scope of this review.

\subsubsection{Antecedents of safety performance - distal person-related factors}

Christian and colleagues' (2009) person-related distal antecedents of safety performance account for individual differences in personality and for differences in job and safety attitudes. These factors have seen relatively little research in the safety field, however both see substantial work in organizational studies and related fields.

\subsubsection{Individual differences and safety motivation}

The safety field's focus on the organizational level constructs of safety climate and safety culture means it is largely disconnected from studies of individual differences, even though human factors are recognized as a significant contributor to most accidents 
(Rasmussen, 1997) and have received consistent academic interest since the 1950s (Clarke \& Robertson, 2008). Much like in the greater work motivation field, the study of individual differences in safety covers a wide range of constructs with no organizing taxonomy. The research is full of contradictory findings, pointing towards both low predictive validity (Barrick, Mount, \& Judge, 2001) and immature methodology (with regards to safety studies) (Clarke \& Robertson, 2005). The majority of safety studies and individual differences look at traffic accidents or computer simulations rather than workplace contexts.

Three recent workplace safety meta-analysis on individual differences has attempted to draw some cohesion to this disparate field. Like the larger work motivation field, the Five Factor Model (FFM) of personality traits has proven a reliable means of organizing this array of work (Moon \& Livne, 2011). The FFM consists of five traits: conscientiousness, associated with planning, achievement, and dependability; agreeableness, which relates to cooperation and trustfulness; emotional stability which captures personal security and lack of anxiety (the opposite end of the emotional stability continuum is neuroticism); openness which refers to curiosity, intellect and creativity; and lastly, extraversion which consists of sociability, ambition, and dominance (Barrick, Mount, \& Judge, 2001).

Clarke and Robertson (2008) found the trait of agreeableness is a valid and generalizable predictor of work accidents $(r=0.44)$. Openness, low conscientiousness, and neuroticism were all positively correlated with accident involvement $(r=0.50,0.31$, and 0.30 respectively) however these results showed such wide variability upon meta-analysis as 
to be unreliable. These findings do not align with the general work motivation field, which finds conscientiousness to be the strongest and most consistent predictor of performance (Salgado, 2013). Clarke and Robertson (2008) found personality to be a better predictor of safety performance in non-restrictive climates where individuality may be expressed. In a separate and earlier meta-analysis, these same authors found only neuroticism to be a significant predictor for occupational accidents $(r=0.28)$ (Clarke \& Robertson, 2005). These contradictory findings were not addressed in the second paper.

Beus, Dhanani, and McCord (2014) looked at FFM's unique variance in unsafe behaviour relative to safety climate. Meta-analysis found that agreeableness $(r=-.26)$ and conscientiousness $(r=-.25)$ were negatively related to unsafe behaviour, while neuroticism $(r=.13)$ and extraversion $(r=.10)$ were positively but weakly related to unsafe behaviour. These results more similarly align with general work performance findings.

Combined, FFM and safety climate account for nearly half $\left(R^{2}=.43\right)$ of the total variance in safety behaviour (Beus, Dhanani, \& McCord, 2014). Safety climate perceptions account for $66.8 \%$ of the explained variance, while personality traits account for $33.2 \%$ (Beus, Dhanani, \& McCord, 2014).

Christian and colleagues' (2009) safety meta-analysis considered FFM traits relative to other personality measures. Similar to Beus et al. (2014) conscientiousness was positively related to safety performance $(r=.18)$, however, locus of control $(r=.35)$, risk taking $(r=$ 
$.28)$, and job attitudes $(r=.25)$ proved more influential (Christian, Bradley, Wallace, \& Burke, 2009).

A wide range of other person-related factors have been studied in relation to safety, although these lines of research are somewhat underdeveloped with only a few papers in each stream. Risk taking is thoroughly examined in behavioural psychology (Westaby \& Lowe, 2005), and Wilde's (1982) risk homeostasis theory popularized individual level risk tolerance, thoroughly adopted by transportation studies. Fewer studies assess individual level risk taking relative to safety performance or safety outcomes, but those that do find a negative correlation between risk taking and safety performance ${ }^{5}$ (Westaby \& Lowe, 2005). Positive and negative affect (PA/NA) has been related to safety performance. As generalized measures of affect, PA is linked to the FFM's extraversion while NA is linked to neuroticism (Eid \& Diener, 1999). In relation to work performance, NA is positively related to occupational injury rates, while PA is positively related to organizational citizenship behaviours and participation (Kaplan, Bradley, Luchman, \& Haynes, 2009).

A promotion and prevention regulatory focus has been shown to be predicted by safety climate and conscientiousness (Wallace \& Chen, 2006). Regulatory focus, in turn, mediates the relationships of safety climate and conscientiousness when using supervisor ratings of productivity and safety performance. Constructs such as these listed here typically address safety only tangentially within a study of work performance in general.

\footnotetext{
${ }^{5}$ Some would argue risk taking is captured in FFM openness and extraversion; likewise, locus of control can also be linked to neuroticism (Christian, Bradley, Wallace, \& Burke, 2009).
} 
Mischel's (1977) situation strength hypothesis applies to safety and the study of individual differences. He argued personality could not be studied in a vacuum, and behaviour was an interaction between individual differences and situational characteristics (Mischel, 1977). Meyer and colleagues (2010) point to how organizational culture and climate play a role in defining situation strength - the logic of which could be extended to include safety (Fugas, Silva, \& Meliá, 2012). From this perspective, safety climate constrains individual differences, a finding Clarke and Robertson (2008) alluded to. This interaction between distal situation antecedents and individual differences has not been examined (Christian, Bradley, Wallace, \& Burke, 2009).

\subsubsection{Attitudes}

Christian and colleagues (2009) included both safety attitudes and job attitudes as distal person-related antecedents to safety motivation and safety performance. These attitudes, they propose, act in concert with personality characteristics to influence safety motivation and safety knowledge. They concluded via meta-analysis, however, that job attitudes were only weakly related to safety performance $(r=0.25)$ (Christian, Bradley, Wallace, \& Burke, 2009). This finding is in line with organizational studies on attitude in relation to job performance (Judge \& Kammeyer-Mueller, 2012).

Judge anchors the attitude field of study, and defines an attitude as "a psychological tendency that is expressed by evaluating a particular entity with some degree of favour or disfavour", while job attitudes are "evaluations of one's job that express one's feelings toward, beliefs about, and attachment to one's job" (Judge \& Kammeyer-Mueller, 2012: 
343). Attitudes provide valenced summaries of the environment, processed spontaneously and automatically (Wilson, Lindsay, \& Schooler, 2000; Preston, 2011). The evaluative aspect of attitudes is the distinguishing feature of the construct, and is relevant when considering safety values.

Early on, Locke (1969) identified a conceptual gap within job attitudes of "correlation without explanation", as in researchers were correlating job attitudes with a variety of variables, but offered little in the way of a model to explain the mechanism of influence (p. 309). Locke went on to propose job satisfaction as an emotional response, a framework that has persisted to today (Rich, Lepine, \& Crawford, 2010; Judge \& Kammeyer-Mueller, 2012). The persisting paradigm within job satisfaction is that a favourable perception of job characteristics, supervisors or coworkers results in positive feelings which will result in 'motivation' or energy towards organizational tasks.

Job satisfaction research typically places it as the dependent variable and organizational outcome when considering latent constructs. For example, school climate, perceived stress, and self-efficacy in teachers is correlated to job satisfaction (Collie, Shapka, \& Perry, 2012). Supporting goal-setting theory, nurses with higher goal attainment measured higher job satisfaction (Gianfermi \& Buchholz, 2011). Pay is consistently found to relate only marginally to job satisfaction (Judge, Piccolo, Podsakoff, Shaw, \& Rich, 2010). 


\subsubsection{Safety attitude}

Safety attitude is a term widely used and rarely defined. Cox and Cox (1991) are attributed with the first measurement of safety attitudes, implying it is a very large composite of attitudes, beliefs, perceptions, and values that employees share in relation to safety. This composite approach seems to hold true today, as 'safety attitude' is left undefined and is applied to most any individual level consideration of safety. It seems to exclude aggregated perceptions or group level constructs such as safety climate and safety culture.

Cox and Cox (1991) mapped employee safety attitudes with five orthogonal factors: personal scepticism, individual responsibility, the safeness of the work environment, the effectiveness of safety systems, and personal immunity or blame. A follow-up study refined these to six key factors that explained an individual's attitudes towards safety: overt management commitment; personal need for safety; personal appreciation of risk; attributions of blame; conflict and control; and supportive environment (Alexander, Cox, \& Cheyne, 1995). The authors recognized that in addition to attitude factors, these factors formed a partial description of the survey site's safety culture. Other authors subsequently lifted these (or similar) factors from the study of safety at the individual level of analysis and used them in considering safety culture at the organizational level of analysis (Guldenmund, 2000). It is an interesting turn that factors devised at one level of analysis are now taken as a given at a higher level of analysis. 
There is a widespread assumption that a more positive attitude towards safety should lead to greater motivation to perform safe behaviours (Fugas, Silva, \& Meliá, 2012; Christian, Bradley, Wallace, \& Burke, 2009) - a 'downstream' causal effect. But as indicated previously, the link between attitude and behaviour is weak (but relevant). In the opposite causal direction, safety attitude is positively related to safety climate (Zohar, 1980), and safety climate is positively related to safety outcomes, between which there needs to be individual behaviour or performance. Safety outcomes and behaviours have been found to, in turn, influence safety attitudes (Stride et al., 2013). From this view, safety attitudes may work with an 'upstream' causal effect: attitudes influences climate, climate influences behaviour, and behaviour influences attitudes. Safety climate is positioned by authors as a "framework for group norms, attitudes and individual behaviours" (Fugas, Silva, \& Meliá, 2012: 470) but climate is at the same time defined by an aggregation of individual attitudes (Guldenmund, 2010). Early work found safety attitude to be a better predictor of safety climate than vice versa (Díaz \& Cabrera, 1997). This attitude/climate/performance cycle is not acknowledged in the safety research.

Behavioural psychology has found that attitudes are not static, and may be able to change with relative ease (Moreland \& Zajonc, 1982), however some attitudes are more enduring. Wilson and colleagues (2000) argue that new attitudes do not replace the old ones, but that both persist as "dual attitudes" (Wilson, Lindsay, \& Schooler, 2000: 101). Implicit attitudes are persistent and enduring while explicit attitudes change according to the situation; static beliefs and changeable ones can co-exist. This approach recognizes context and the role the environment plays in shaping beliefs, as well as the 'persistent 
and resistant' aspects of the person, introducing values as a part of the evaluative nature of attitudes. Attitudes have not been considered with this sophistication in the safety field, as safety attitudes are defined as an aggregate of attitudes, beliefs, perceptions, and values surrounding safety. However, Beus and colleagues (2010) and Stride and colleagues (2013) have data elluding to the changeable nature of safety attitudes. Given the broad definition of safety attitudes, it is difficult to interpret these findings. Values, as a specific factor within this definition of safety attitudes, are not addressed in the safety literature (Ford \& Tetrick, 2008: 1481), exposing a substantial gap.

\subsubsection{Summary of integrative model of individual safety performance}

By organizing individual safety research into a model based upon antecedents, determinants, and components, Neal and Griffin (2004) and Christian and colleagues (2009) provide a framework by which to consider proximal and distal influences upon individual safety performance. Individual safety performance is most strongly related to the proximal determinants of safety knowledge and safety motivation, and safety motivation is typically found to be more strongly related to safety participation while safety knowledge is more related to safety compliance (Griffin \& Neal, 2000; Vinodkumara \& Bhasib, 2010; Neal \& Griffin, 2006). Why safety participation is found to be more influenced by social factors than safety compliance is not explained in the literature.

As distal antecedents, the FFM and safety climate account for nearly half of the total variance in safety behaviour. Safety performance is moderately related to locus of control 
but only weakly related to conscientiousness and risk taking. Locus of control is also stronger in relation to safety participation than to compliance. Job attitudes are weakly related to safety performance.

The direction of the causal arrow is up for debate in these relationships, as safety participation could conceivably improve safety motivation (Neal \& Griffin, 2006), among other reverse relationships. There is also some indication that safety outcomes influence safety climate more than safety climate influences outcomes (Beus et al., 2010), and that observations of coworkers' safety performance influences future safety participation, compliance, and motivation (Stride et al., 2013).

Missing from Neal and Griffin (2004) and Christian and colleagues' (2009) model of individual safety performance is one or several mechanisms. Individual authors have tentatively proposed a small variety of mechanisms (reviewed below), but this model does not address means. Likewise, a feedback loop is required, as findings show that safety performance influences future behaviour, which works its way upstream to inform collective perceptions of safety climate and ultimately inform culture.

Peer influence is also missing from this integrative model. A multi-level climate construct includes a group level of analysis, but within climate factors only supervisor influence is considered. As Christian and colleagues' (2009) model is based upon a meta-analysis, the emerging work on social influence may be too new to be reflected in it.

Lastly, values are absent in this model and in safety research in general. Safety culture, climate, and safety attitudes all imply a value appraisal of some kind - completely 
unmentioned in safety studies. Values form the core of cultural assumptions (Schein, 1985; Hofstede, 1990), and can offer substantive explanation of differences between groups or individuals (Pidgeon, 1998). Internalization is about the adoption of these values and priorities.

These missing items of mechanisms, peer influence and values will be addressed in further portions of this literature review.

\subsubsection{Cognitive mechanisms in the study of individual safety}

The vast majority of safety research is targeted towards the nature and quality of antecedents of workplace safety. Much less research has explored the mechanisms for such influence (Törner, 2011). When mechanisms are discussed, it is via tentative speculation to explain empirical results. Connection between findings and mediating theory is poorly defined.

Andriessen (1978) was the first to attach Vroom's (1964) expectancy theory to safety behaviour. At a time when systemized workplace safety was a relatively new concept to the blue-collar worker, Andriessen's (1978) focus was on the valence of safety outcomes and instrumentality between safe behaviour and outcomes. Other research has relied upon operant theory focussing on behavioural consequence and reward (Grindle, Dickinson, \& Boettcher, 2000). Self-regulation constructs such as expectancy theory and Wilde's (1982) risk homeostasis theory represent a parallel tact (Ford \& Tetrick, 2008). What all of these safety behaviour interpretations have in common is a cognitive, agency based approach (Ellemers, de Gilder, \& Haslam, 2004). They assume that individuals are 
goal oriented and plan their actions (Ford \& Tetrick, 2008), as opposed to react or respond based upon possible alternative interpretations of motivation. Workers have the ability and wherewithal, it is assumed, to decide to act carefully or carelessly on a moment by moment basis; it is assumed workers can choose to improve workplace safety or ignore hazards that pose a risk to coworkers (Choudry, Fang, \& Mohamed, 2007). Below is an overview of the mechanisms that are present in safety research, and how those mechanisms are addressed.

\subsubsection{Operant theory}

Behaviourist approaches based upon operant theory are one of the "seminal perspectives" on safety (Ford \& Tetrick, 2008: 1474), and formed much of the early safety research agenda. Operant theory explains the relationship between behaviours and their reinforcing consequences: actions that produce positive outcomes will be replicated while those that produce negative outcomes will be discarded (Skinner, 1953). From a safety perspective, negative outcomes are recognized as being more than resultant injury; production slowdown, discomfort from safety equipment, or coworker reaction or rejection are all considered consequences of unsafe behaviour (Reason, 1998).

Behavioural safety interventions identify behaviours that impact safety, train and monitor those behaviours, and then institute reinforcement structures to promote the desired behaviour. In their review of the relevant literature, reinforcement strategies have been proven to be effective in improving safety (Grindle, Dickinson, \& Boettcher, 2000). Sulzer- 
Azaroff and Austin (2000) found direct evidence that behaviour based approaches reduce injuries.

As an example of this type of safety research, Laitinen and Ruohomäki (1996) inventoried construction safety practices such as fall protection, machine safety, PPE and scaffolding use. By creating a safety index, the authors could assess safety improvement from $60 \%$ compliance to $90 \%$. Reber and Wallin (1984) overview the wide variety of behaviour oriented safety practices being tested at that time. Feedback, collaborative goal setting, competition, incentives, praise, contingent control, and social reinforcement all received academic attention.

Successful outcomes in this area of research informed the 'engineered' safety culture perspective - i.e. by inserting certain workplace cultural or climate traits positive behaviours will follow. Reason (1998) anchors this school of thought, recognizing "It is hard to change the attitudes and beliefs of adults by direct methods of persuasion. But acting and doing, shaped by organizational controls, can lead to thinking and believing" (Reason, 1998: n.p.).

Reinforcement strategies are rationalized as necessary because "natural contingencies do not always reinforce safety. Injuries, given their relative rarity, are not always a likely outcome of even the riskiest of behaviour, while the negative consequences of safe behaviours, including discomfort and production slowdown, are often certain" (Ford \& Tetrick, 2008: 1475). Although injury may be a punishing consequence, it is statistically improbable that it will be realized (Grindle, Dickinson, \& Boettcher, 2000: 33). 
Findings emerged both in safety and motivation studies that undermine operant theory. Discontinued safety rewards found no drop off in performance (Al-Hemoud \& Al-Asfoor, 2006). Grindle and colleagues (2000) found that 5 of 6 safety programs showed continued performance 6 months after the programs were withdrawn. Operant theory would predict otherwise. Training alone has been found to significantly improve safety motivation and safety performance (Vinodkumara \& Bhasib, 2010) with no performance based reinforcement required. The safety climate field has extensive findings that concur (Zohar, 2010).

Like all behaviourist approaches, the cognitive aspects or processes preceding action are not addressed - no specific mechanism is identified to account for its success. This critique is as true for operant theories such as the very successful goal setting research stream as it is for safety (Latham, 2012). What motivates the individual to pursue a certain goal or perform safely is left unspecified. Behaviourist approaches also ignore the organizational and social context in which the individual works, relevant as it is the "antecedent to enforcement" (Ford \& Tetrick, 2008: 1475).

\subsubsection{Self-regulation}

Self-regulation constructs get closer to the motives underlying safety motivation, providing an explanation for an individual's willingness to exert effort to enact safety behaviours and the valence associated with those behaviours. In these models, safety is discretionary, and workers choose to perform safely when it aligns with their individual goals - a human agency model of behaviour (Ford \& Tetrick, 2008). 
Self-regulation is defined as internal processes that guide goal-directed activities over time, and implies modulation of thought, affect, behaviour and attention (Karoly, 1993). Self-regulation is a "proximal motivation process, as it influences task performance by determining the attention resources devoted to a task" (Le, 2011: 116). The work motivation field utilizes self-regulation to explain goal choice and behaviours that contribute to goal striving. For these reasons, it has obvious application to safety performance, even though it is often left unspecified.

\subsection{Expectancy theory}

Vroom's (1964) expectancy theory predicts an individual's effort towards a task is based upon a function or probability that one's effort will be successful (expectancy and instrumentality), and the importance of the intended outcome to the individual (valence). From a safety perspective, expectancy theory predicts that workers will be motivated to perform procedures or participate in safety initiatives if they believe that those behaviours will lead to valuable outcomes (Neal \& Griffin, 2006). Safety climate studies rely upon expectancy theory extensively, using it to attach organizational priorities to individual behaviour (Zohar, 2000). Climate creates expectancies around desired safety behaviours, which informs workers' understanding of safety valence and priorities. Supervisors who monitor and enforce safety should generate an expectancy valence associated with safe behaviour (Zohar, 2000). Safety culture's organizational values rely upon expectancy theory to translate those down to individual valence associated with safety (Guldenmund, 2010). It is important to note that almost all of this is implied, as mechanisms are often not addressed in safety research. When mechanisms are 
addressed, they are typically underspecified, perhaps only obliquely referred to such as worker's 'value' placed upon safety. Ford and Tetrick (2008) point to a number of models of personal safety that rely upon a value judgement of relative risk to relative reward, in which valence and expectancy are assumed.

Expectancy theory can explain the link between safety knowledge and safety compliance behaviour (outlined previously). Increased knowledge builds awareness of expectancy and instrumentality. According to expectancy theory this will lead to greater propensity to perform these tasks. Some of the behaviourist school's safety findings can be explained via expectancy theory: Zohar and Fussfeld (1981) found workers continued to wear ear protection after a safety program was discontinued, with one potential explanation being workers grew to appreciate (and thus adopted) the safety measures based on a learned valence of ear protection utility. Authors in the safety field rely upon expectancy, but do not acknowledge it, or often, any other mediating mechanism.

\subsection{Risk homeostasis}

Risk homeostasis theory forms a practical application of expectancy, built around expectancy and valence. Wilde (1982) proposes all individuals have a target level risk, and will behave to exercise that target level. If safety precautions are put into place, individuals will behave in ways that increase or decrease the risk to their target level. This theory was derived from traffic statistics, finding that engineered safety features (such as anti-lock automobile brakes) resulted in faster driving and tailgating, negating the safety effects of such features (Wilde, 1982). This theory has gained wide acceptance in traffic, 
engineered safety, and risk taking studies in general. Implied in this theory is the expectancy and valence of exercising one's target level risk. Risk taking behaviour is a significant contributor to workplace accidents (Johnson, Singh, \& Young, 1998), and workers' target level risk can be considered a controlling factor in safety performance (Lingard \& Holmes, 2001).

Despite the theory's premise, engineered safety features have been proven effective in many circumstances. While behaviours may increase towards target level risk, it often does not completely offset the benefits of the safety precautions (Ford \& Tetrick, 2008). That coworker risk taking is a relatively strong predictor of young worker's risk taking (Westaby \& Lowe, 2005) suggests the role of social influence. This same finding can be applied to expectancy theories in general; the valence one attaches to a desirable outcome has cultural and social influences.

Cognition and conscious thought were originally an assumption within self-regulation constructs (Bandura \& Locke, 2003), but modern understanding now includes subconscious and automatic responses that also fall within the definition of selfregulation (Bargh, Gollwitzer, Lee-Chai, Barndollar, \& Trotschel, 2001). These are only recently being applied to safety studies (Xu, 2014).

\subsection{Higher order goals}

Beus, Dhanani, and McCord (2014) introduce a significant contribution to safety studies by tying individual differences in safety behaviour to higher order goals. Barrick, Mount, and Li's (2013) theory of purposeful work argues that individual differences in personality 
motivate behaviour towards implicit higher order goals. According to the theory of purposeful work, the FFM personality traits predispose individuals in varying degrees to strive for the higher-order goals of communion, status, autonomy, and achievement (Barrick, Mount, \& Li, 2013). Based upon a wide range of motivational theories (Maslow, 1943; McClelland, 1961; Gagne \& Deci, 2005), higher order goals are a universal motivating force driving individual work behaviour. Beus and colleagues (2014) write, "As a context-specific application of Barrick et al.'s theory, we posit that safety-related behaviours - whether safe or unsafe - can be instrumental in attaining higher-order goals and that motivation to attain these goals drives worker behaviour" (Beus, Dhanani, \& McCord, 2014: 5). This makes Beus and colleagues' (2014) paper one of the few modern papers to explicitly tie safety studies to a greater organizational work theory, and the only paper to address implicit higher order goals - a more deliberate and specified approach to understanding safety than a generic expectancy model.

Overall, the mechanisms assumed in safety research tend towards an agency based expectancy model. While operant theory based research proves safety behaviour programs can be successful, these approaches lack a theoretical basis. Self-regulation views on safety behaviour rely upon a worker's cognitive choice to be safe or unsafe, ignoring alternative motivators of action that the wider motivation field has explored. The source of one's valence - the source of one's value appraisal - is left unspecified in expectancy safety models. This leaves open a door to examine social influence and the role of coworkers in influencing individual safety values and the internalization of safety. 


\subsection{SAFETY RESEARCH WITHIN INDUSTRIAL SECTORS}

The sampling frame for this research study utilizes three sectors within safety critical or 'process safety' industries. Process safety industries have a core task of producing safety, hence these are also known as safety critical industries. While worker safety is a legislated obligation of every employer, only a select few occupations have as their core task producing safety or protecting the public's safety as their primary function.

Firefighters, paramedics, and whitewater river guides all share a core task of producing safety. Firefighters put themselves in harm's way in order to fulfill their duties of civic protection. Paramedics, similarly, are tasked with responding to others' medical distress, often in potentially harmful environments. Whitewater river rafting guides take clients to inherently dangerous environments for thrills, but are tasked with protecting their clients from excessive exposure to said environment. Safety is a key aspect to each of these industries' core tasks. What follows is a brief overview of each sector's safety literature, with a focus upon individual safety behaviour.

\subsubsection{Firefighting safety studies}

Firefighting has several journals dedicated to fire safety engineering, examining technological practice or safety equipment testing. Typical of wider safety studies, there are also a number of studies examining the correlates of work related injuries, typically utilizing objective variables (Moore-Merrell, Zhou, McDonald-Valentine, Goldstein, \& Slocum, 2008) and only rarely incorporating behavioural aspects of safety (Smith \& DeJoy, 2014). 
Safety culture has come to firefighting studies relatively late, with work still being put into understanding the parameters of safety culture relative to firefighting (Pessemier, 2008) and articulating an operating definition (Pessemier \& England, 2012). With this more recent adoption of safety culture comes a more sophisticated view relative to other industrial applications of safety culture, with work like Pessemier (2008) integrating the role of individual self-identity within organizational identity and safety culture.

Firefighting is often used as a proxy for larger organizational studies, with Weick's (1993) famous Mann Gulch Disaster the most recognizable, using a wild land fire response as an example of organizational breakdown. Firefighting has also been used to study organizational communication (Thackaberry, 2004) and social construction (Barton \& Sutcliffe, 2009), for example. At the individual level of analysis, human factors is well entrenched as a research paradigm. Fire safety uses the human factors model to focus on decision making in dynamic environments (Omodei, McLennan, \& Reynolds, 2005). Even given the human factors research stream, there is no work in the firefighting sector that looks at individual safety motivation or peer influence. Although not safety related, a paper by Grant (2008) surveyed firefighter prosocial motivation (a concept not distant from coworker influence), finding intrinsic motivation moderated prosocial behaviour (volunteer overtime shifts). Grant (2008) used self-determination theory to connect his argument, similar to what this present research study utilized as a conceptual foundation, detailed in the research model below. 


\subsubsection{Paramedic safety studies}

There are tens of thousands of studies involving paramedics. All but a fraction of these are clinical studies measuring paramedics' medical intervention techniques, medication efficacy or patient outcomes. These studies all appear in medical journals. For example, there are over a thousand studies assessing the success rates of intubating patients as a means of pre-hospital airway management. There are an equal number of studies examining the clinical efficacy of different medical assessment decision tools.

A 2012 literature review of the paramedic field found just seven articles that examined the 'non-technical' aspects of paramedicine i.e. social and cognitive aspects of the work (Shields \& Flin, 2012). These studies focussed on the paramedic/patient relationship and on individual paramedic decision making. There is no peer reviewed work looking at the paramedic partnership or coworker dynamic.

A study that peer ranked paramedic professional behaviours listed as number 10 'teamwork/diplomacy' behind a list of individual traits such as empathy and time management skills (Brown, Margolis, \& Levine, 2005). 'Teamwork/diplomacy' is not defined in this study, however the term 'teamwork' emerges in other paramedic centred work, and is defined as the paramedic working in chain of patient care. The 'team members' include emergency room nurses, doctors and eventual outpatient services (Tavares, Bowles , \& Donelon, 2016), most of whom a paramedic would have very little personal contact with. Post-traumatic stress is a current topic in the paramedic field. In the dozens of studies involving paramedics, only one article places coworker support as a 
relevant variable (Regehr \& Millar, 2007). The partner or coworker paradigm is absent in paramedic research.

\subsubsection{River guide safety studies}

Commercial whitewater rafting and the individuals who work in that sector have received very little academic attention. Like the two process safety/safety critical industries reviewed above, river rafting accident data has been studied specifically (Hunter, 2007; Whisman \& Hollenhorst, 1999; Attarian \& Siderelis, 2013) or aggregated into the larger adventure tourism industry's accident data (Bentley, Page, \& Walker, 2004). Whitewater rafting is more likely to be found in studies regarding the sociology of adventure or of risk, but from the perspective of the participants/passengers. Likewise, a modest number of papers examine river rafting as an "extended service encounter" (Arnould \& Price, 1993) and from a consumer research or tourism management standpoint.

Regarding those who work as river guides, only two papers are found (beyond injury data): one examining guides as involved in emotional labour (Sharpe, 2005), and a second ethnography looking at identity creation (Holyfield \& Jonas, 2003). While neither paper considers safety values or motivation, the latter paper does conclude river guide identity includes constructing self-identity markers of competence and separate constructions of perceptions of risk, either of which could be tied back to individual internalization of safety. 


\subsection{SUMMARY OF THE SAFETY LITERATURE}

Safe behaviour requires a particular set of attitudes and values to motivate it. Most safety research skirts this issue. As this research project examined the influence of coworkers upon the individual internalization of safety, the emergent peer influence safety literature is of the most interest. Internalizing safety, however, is placed within a larger individual level safety field of study.

The review of literature organized the varied safety literature by recognizing components, determinants, and antecedents of safety outcomes (Christian, Bradley, Wallace, \& Burke, 2009). As proximal components of safety performance, compliance and safety participation have a strong positive relationship with individual safety performance. Of the two, safety participation has been found to be more influenced by coworker descriptive norms. Compliance is more affected by supervisory oversight.

As distal determinants of safety compliance and participation, safety motivation has recieved the majority of research interest compared to safety knowledge, as the latter is considered to be somewhat constant due to training. Safety motivation is the most prominent mediator between (organization level) safety climate and (individual level) safety behaviour.

The distal situation- and person-related factors are varied and only loosely related to the much larger organizational or psychology fields from which they were drawn. Of these, safety climate has and still receives the lion's share of academic interest, likely due to its "robust" predictive ability in regards to safety outcomes, compliance, participation, and 
safety motivation (Zohar, 2010: 1517). The parallel study of safety culture has yet to produce such definitive results. Leadership is found to be both a mediator between safety climate and safety performance as well as have a stand-alone moderating effect. The mechanisms or process by which situation related antecedents influence individual safety motivation is only tentatively hypothesized as any of expectancy, social exchange, or sensemaking.

Person-related antecedents of safety performance fall into either individual differences (personality) or attitude. Regarding these, locus of control, risk taking, and job attitudes all showed greater influence upon safety performance than any of the factors in FFM. The FFM has gained traction in the safety field but its results have proven highly variable. No one factor consistently outweighs its opposing factors.

Attitudes have been proven a relevant contributor to behaviour (Judge \& KammeyerMueller, 2012). Safety climate is defined by an aggregation of individual attitudes but safety attitude is found to be a better predictor of safety climate than vice versa. Attitudes may play a more influential role in safety behaviour, however basic issues such as causal direction have not been sorted. Attitudes have not been considered with any sophistication in the safety field, however Beus and colleagues (2010) and Stride and colleagues (2013) present data elluding to the changeable nature of safety attitudes. Values are not addressed in the safety literature.

The psychosocial aspects of safety have only recently become a research agenda item, with coworkers recognized as an "important yet often overlooked social influence upon 
safety" (Tucker et al., 2008: 322). This emergent research finds coworkers to be more influential upon individual safety motivation than the supervisor or organization level safety climate.

An important meta-analysis by Chiaburu and Harrison (2008) on coworker effects provided the first empirical support indicating that coworker actions predict perceptions, attitudes, and behaviour outcomes of their colleagues even when accounting for the influence of the direct supervisor. Brondino, Silva, and Pasin (2012) picked up on this work and tested a multi-level safety climate model that included the overlooked coworker element. These authors found that coworker safety climate had a stronger mediating role with safety behaviour than did supervisor safety climate. Multi-level structural equation modelling pointed to coworker safety climate as reducing the effect of the supervisor safety climate - a finding uncovering substantially more complexity in the formation of safety climate than the predominant hierarchical safety climate paradigm. Other researchers have examined threads within this emergent area (Jiang, Yuc, Li, \& Li, 2010; Westaby \& Lowe, 2005; Zhou, Fang, \& Wang, 2008), but the work of Chiaburu and Harrison (2008) has opened a new stream of research for the safety field. The authors recognize that the missing link is the mechanism or processes underlying these direct relationships that they uncovered (Chiaburu \& Harrison, 2008: 1095).

Norms are one means by which to explain coworker influence. In the safety field, descriptive norms are found to be more influential than injunctive norms, a finding at 
odds with greater social psychology findings. What is it about safety that makes it more influenced by what others actually do than by what others believe ought to be done?

The theory of planned behaviour is a more sophisticated dual process theory of motivation that relies upon norms as well as individual attitude and perception of control of outcomes (Ajzen, 2001). This theory offers the most thorough and convincing mechanism by which coworkers influence individual safety behaviour, although its subconscious or 'nondeliberative' effects are yet to be explored. Social exchange, social learning, and cognitive dissonance all appear as alternative explanations of peer influence in the safety literature.

Norms appear as a unifying theme in all of these explanations. The study of norms recognizes that group social identity can generate powerful group values, but where these group values come from is not made clear, nor their relationship to individual values.

\subsubsection{Reviewed safety variables not considered for further study}

Christian and colleagues (2009) organized the antecedents to individual safety motivation into distal situation-related factors and person-related factors. Of these two categories,

the research question that guided the present study was intended to build upon a particular aspect of the under-developed group level situation-related factors. Omitted from further study are the distal person-related factors. These person-related factors include a number of variables identified in the literature review. 
Safety studies have pursued all of locus of control, risk taking and the FFM as individual differences in safety performance. Locus of control and risk taking show greater influence upon safety performance than the FFM, but locus of control and risk taking studies are often experimental in approach rather than work based, and can be argued to be present in the FFM factors of neuroticism and extraversion, respectively (Christian, Bradley, Wallace, \& Burke, 2009). Certain sectors such as transportation have thoroughly examined risk taking, but that has not translated over to work based safety studies. Despite its lesser influence, the FFM has gained predominance in safety studies but results have proven highly variable. The FFM is a well-established construct outside of safety studies, with reliable measures and consolidated findings. Further FFM research in safety studies will likely converge with the established findings in the greater FFM field: extraversion is linked to happiness and success in social settings such as work, neuroticism is linked to negative responses in general, and conscientiousness remains the most predictive trait related to work based motivation (Brown, Lent, Telander, \& Tramayne, 2011; Denissen \& Penke, 2008). Additionally, and perhaps explaining the various results in safety studies, the FFM shows consistently low correlations to observed behaviour (Latham, 2012). There is little evidence to suggest that personality-based individual differences will offer any significant insight into safety behaviour, especially with regards to workplace and social settings (where striving for status and striving for affiliation "consistently emerge" (Barrick, Mount, \& Judge, 2001: 25) as mediators upon personality). It is for these reasons that individual differences in personality were not pursued in this research. 
Christian and colleagues (2009) distal situation-related factors offer an alternative path of study encompassing safety climate and supervisory leadership. Both variables have a strong safety specific body of work behind them. However, the present study pursued an alternative interpretation of the group level within climate and culture by examining the emerging role of the coworker, recognized as an "important yet often overlooked social influence upon safety" (Tucker et al., 2008: 322). This research project operationalized safety climate's group level variable to consider coworker influence, rather than the existing supervisory paradigm. Emergent research found coworkers as more influential upon individual safety attitudes, values, and motivation than the supervisor or organization level safety climate. For these reasons the traditional situation-related antecedents of leadership and climate were not (specifically) pursued. Newer safety research findings place coworkers as an additional situation-based antecedent, not present in Christian and colleagues (2009) integrative model of individual safety.

\subsubsection{Gaps in the literature}

When comparing the state of individual level safety studies to the larger fields of workbased motivation or social psychology, several substantial avenues of study have not yet been explored relative to safety. The prominent role that emotion and emotion management play in psychology and organizational behaviour is more or less untouched by safety studies ${ }^{6}$. Likewise, the roles of the subconscious, automaticity and dual process theories are yet to make any real presence in safety literature. The theory of planned

\footnotetext{
${ }^{6}$ Patterson, Bradley, \& Artiss (2000) are an exception with their study of the role of emotion in operator error.
} 
behaviour as it is understood in social psychology utilizes both a conscious/deliberative aspect when considering the influence of norms, as well as a subconscious/nondeliberative aspect (Manning, 2011). TPB has not reached this level of sophistication yet in safety studies. Individual level safety is still predominantly viewed as a deliberate, cognitive construct.

Safety research is behaviourist in approach, oriented around measurable output and behaviours. However individual level safety values or motivation don't produce any objective output (on their own), leaving them implied, inferred or overlooked in safety research.

The literature reviewed above identified some unanswered questions and not as-yet pursued lines of inquiry. That values are absent from safety studies produces the largest gap - a fact recognized by academics (Schwartz \& Boehnke, 2004; Christian, Bradley, Wallace, \& Burke, 2009; Ford \& Tetrick, 2008). As a distal person-related antecedent to safety, safety attitude relies upon an evaluative judgement, which would derive from individual values. More abstractly, safety in and of itself is a value statement, implying the relative importance of certain inputs, behaviours, and outcomes over others. Beus, Dhanani, and McCord's (2014) introduction of safety as satisfying needs or higher order goals approaches values, but is only a thread of a large and longstanding area of motivation research yet to be tapped.

There are untested assumptions regarding the causal direction of organizational climate to individual motivation to performance. Climate is constructed of individual perceptions 
and attitudes but also informs individual perceptions and attitudes (Neal \& Griffin, 2006). This cyclical nature of the climate construct is not addressed.

More important for this research project are questions regarding the role that social norms play in individual motivation. The research findings that safety participation is more socially influenced than safety compliance has not been further examined. Likewise, safety motivation is found to be socially influenced, but why this is or why safety knowledge is not socially influenced is left unexplained. Contrary to other fields of study, safety research finds descriptive norms to trump injunctive norms. Why is coworkers' actual behaviour more influential than what they say or believe? Does this somehow relate back to the social aspects of safety participation and safety motivation?

Overwhelmingly, the mechanisms by which individuals are influenced by safety referents is left unexamined - it is the proverbial elephant in the safety studies room. Speculative explanations are offered, but those are rarely tied to the study's (often quantitative) data. This gap is the basis for the research question guiding this study: What is the influence of coworkers in individual internalization of safety? 


\section{RESEARCH MODEL AND CONCEPTUAL FRAMEWORK}

This research study was exploratory in nature looking for mechanisms of influence in an as-yet unstudied relationship between coworkers and the individual internalization of safety. It was built upon recent findings that individual safety values, attitudes and motivation are greatly influenced by those one works with. This research examined the individual internalizing of safety, but relied upon social constructs of norms and group influence. The unit of study is at the individual level of analysis. Based upon a review of the literature there was an assumption, which was the phenomenon under study in this research, that a group's orientation towards safety is transferred to and internalized by the individual as individuals recognize and adhere to work safety norms. The research question becomes: What is the influence of coworkers on the internalization of safety?

The qualitative approach of this research was inherently post-positivist. Contextual patterns (between, in this case, coworkers' safety beliefs and the individual's cognitions and internalizing the value of safety) potentially reflected or projected deeper underlying structures or mechanisms (in this case group safety beliefs and norms) (Myers, 2009). From a methodology standpoint, a post-positivist approach is one intended to "to carry out intensive studies of a small number of cases in order to retrieve through analysis the underlying patterns that are arguably reflected in the surface structures" (Alvesson, 2010: 19). Qualitative research was the most appropriate means of exploring these contextual patterns. Whereas quantitative research seeks to measure and assess the relationships between variables (which regarding coworker influence upon safety remain undefined), 
a qualitative approach attempts "to explicate the ways people in particular settings come to understand, account for, take action, and otherwise manage their day-to-day situations" (Miles, Huberman, \& Saldana, 2014: 7). A qualitative approach allowed findings to be reported via "thick descriptions that are vivid and nested in a real context, and have a ring of truth that has strong impact on the reader" (Miles, Huberman, \& Saldana, 2014: 10). Miles and colleagues (2014) infer that the qualitative paradigm holds that multiple participant meanings rather than a single, objective truth exists.

Exploratory research is typically inductive (Singleton \& Straits, 2010), as was this research. However, this research utilized a more structured approach than sometimes employed in qualitative research. Informed by a thorough literature review and theoretical connections offered by underutilized (in safety studies) social motivation theories, propositions were formed to guide this study. This new research was interpretive rather than empirical, as exactly what to measure in the relationship between coworkers' safety beliefs and the individual is as yet unspecified, and the data collection was centred upon the social constructions of the informants.

The problem being pursued in this research study was important. Most directly, this work contributes to the emergent coworker influence field of study and addressed a gap in the safety literature by addressing coworker influence and addressing beliefs and values. Pratt, however, points out that identifying a gap is not enough to justify research and that additional justification is required to explain "why it is important to fill this gap" (Pratt, 2009: 858). The growing understanding of individual safety motivation and safety 
behaviour point to it being a complex and sophisticated phenomenon. At the same time, especially for safety critical industries, it is of great importance. This research sheds some light on one aspect of this psychological complexity by addressing social influences upon the individual. Potentially, this research on safety has application to the greater study of motivation, social influence and to the field of organizational culture.

\subsection{THEORETICAL CONNECTIONS AND GUIDING PROPOSITIONS}

What is the influence of coworkers upon the individual internalization of safety? It has long been recognized in sociology that one of the most pervasive determinants of individual behaviour is the influence of those around a person (Burnkrant \& Cousineau, 1975). Recent work by Chiaburu and Harrison (2008) solidify the influential role coworkers play in shaping individual values, attitudes, and motivation. Emerging safety specific work by Brondino et al. (2012), Jiang et al. (2010) and Zhou and colleagues (2008) point to the same conclusion. The research question guiding this project builds upon these works and asks how do coworkers influence the individual with regards to safety? What role do coworkers play in the individual internalizing beliefs regarding safety? To this end, a theoretical model to explain coworker influence upon the internalizing of safety beliefs or values was proposed. The adoption of social values played a pivotal role in the explanation. This model provides an alternative to the limited explanations offered by safety studies to date regarding the mechanisms of peer influence upon safety.

The following section outlines the theoretical rationale underlying this project's explanatory model and guiding propositions. These propositions provided structure to 
this qualitative inquiry. Miles, Huberman, and Saldana (2014) define a proposition as "a statement that puts forth a conditional event - an if-then or why-because proposal that gets closer to prediction or theory" (Miles, Huberman, \& Saldana, 2014: 99-100). This is in contrast to a hypothesis, defined as an expected but unconfirmed relationship among two or more variables (Singleton \& Straits, 2010: 589), or more specifically a "testable proposition that purports to explain a phenomenon" (Myers, 2009: 259). Given that the variables under examination in this research project were emergent, and that the potential relationships and mechanisms were yet to be explored in the literature, the

empirical 'testability' of any hypothesis was premature. From this perspective, the present research was more closely aligned to an exploratory study as opposed to an explanatory one. There are, however, existing theories of social influence which provide some insight, and from which were deduced a series of propositions. These propositions structured and guided the research, common practice in nascent theory and in attempting to explain emerging phenomenon (Edmondson \& McManus, 2007).

\subsubsection{Normative rules and values}

Individuals behave in line with normative expectations (Manning, 2011) in order to satisfy the basic need to belong (Fiske, 2003). Unlike formal organizational structures such as culture and climate, norms represent informal and social aspects of influence, generated by workers themselves (Ehrhart \& Naumann, 2004; Barker, 1993). These normative expectations are found to be influential upon safety behaviour (Johnson \& Hall, 2005; Fogarty \& Shaw, 2010; Zohar D. , 2000; Fugas, Melia, \& Silva, 2011), and more specifically influential upon individual safety motivation and safety participation. 
One view of norms (and by default coworkers) in safety science is that they serve as a model of acceptable behaviour. Fugas, Melia, and Silva (2011) propose that when a number of group members regularly perform specific safety behaviours, these behaviours then become recognized as typical. This role model effect relies upon the descriptive aspect of norms (Fugas, Melia, \& Silva, 2011; Fogarty \& Shaw, 2010; Chiaburu \& Harrison, 2008; Jiang, Yuc, Li, \& Li, 2010). Social learning theory uses norms in the same way - by observing and learning from others, parameters of acceptable behaviour are established (Bandura, 1977). Stride and colleagues write "regardless of organizational prescription, employees may learn safety norms from the unsafe behaviour of their coworkers" (Stride et al., 2013: 47-48). In this light, coworkers are positioned as 'role senders' (Chiaburu \& Harrison, 2008). This interpretation of the influence of norms is a relatively passive, externalized view.

Barker (1993) provides a powerful alternative view of norms. He observed the concertive control that coworker-developed, value-based, normative rules impose. Via field observation, Barker watched newly formed self-managing teams transform initial sensemaking discursive into value consensus, which then became norms, and which eventually became powerful, socially enforced rules. This experience of norms is more than the academic category of injunctive norms - what one 'ought to do' (Fugas, Melia, \& Silva, 2011; Lingard, Cooke, \& Blismas, 2011). Instead this is the true 'normative' experience of norms, where social rules are forcibly applied to in-group members (Cialdini \& Goldstein, 2004). This normative aspect of social rules appears to be most influential 
regarding socially motivated actions, where one's role or status in the group may be compromised (Lapinski \& Rimal, 2005; Manning, 2011).

It is by this avenue of group-generated normative influence that beliefs and values can be imported into the context of coworker influence and by extension into individual safety motivation. While norms are situation specific, values are general in nature and guide behaviour and cognition via 'trans-situational' goals (Van Dijk \& Kluger, 2004). More specifically, Hitlin and Piliavin define values as “(a) Concepts or beliefs, (b) about desirable end states or behaviors, (c) that transcend specific situations, (d) guide selection or evaluation of behavior and events, and (e) are ordered by relative importance" (Hitlin \& Piliavin, 2004: 362).

The inherent cultural and social aspect of values points to values being a learned phenomenon (Hitlin \& Piliavin, 2004). Early and influential work by Rokeach (1973) set the stage for this observation. He found that when one's value position on a particular subject was contrary to one's peer group, the value was suppressed or even changed to match the group's (Rokeach, 1973). Since that time, research has described values as somewhat malleable (Cable \& Parsons, 2001; Lauver \& Kristof-Brown, 2001), depending upon one's openness to change (although this is not without debate, see Jin and Rounds (2012)).

The relationship between safety culture and individual performance relies upon values as a linking concept. More specifically, safety culture represents social system values which should influence individual values (Guldenmund, 2000; Choudry, Fang, \& Mohamed, 
2007). Work performance is found to be highest where there is alignment between individual and organizational values (Wright \& Pandey, 2008) - the logic from which could extend to safety performance. Safety culture functions as both a constraint on individual action and the context within which one acts (Choudry, Fang, \& Mohamed, 2007). In this way, culture serves as a sensemaking lens as well as a normative tool (Guldenmund, 2000).

Safety culture is typically conceptualized and aggregated at the highest organizational level. Both logic and research point to 'cultures' residing at multiple levels within an organization, and one organization-wide safety culture may either not exist (Perrow, 1999) or is made up of many sub-group cultures (Glendon \& Stanton, 2000). Work in the nuclear industry identified coworker related safety culture factors and facets as the most influential to the larger organization's safety culture (Garcia-Herraro, Mariscal, Gutierrez, \& Toca-Otero, 2013).

Linking this material together, coworkers may assemble their collective individual perspectives regarding safety into a group safety culture, one which reflects collective beliefs and values and is manifested in norms. These norms become both context and a constraint upon individual action.

Proposition 1: Coworker safety norms will represent agreed upon group safety values.

\subsubsection{Social identity and self-concept}

In regards to group safety beliefs, social identity theory proposes that individuals categorize themselves according to the social groups of which they consider themselves 
a member (Tajfel, 1974; Haslam, 2004). In some settings individuals pursue their own goals and identify first and foremost as ' $\mathrm{l}$ ', or in another setting can view themselves as a member of a group, and identify as a part of a collective 'we' (Ellemers, de Gilder, \& Haslam, 2004). This has been called the 'paradox of identity', and indicates the constant tension surrounding social identity in groups (Murninghan \& Conlon, 1991). Social identity theory goes on to describe how situational factors can influence this identification between individual or group membership (Ellemers, de Gilder, \& Haslam, 2004). Work settings with high task interdependence (Stark, Shaw, \& Duffy, 2007), for example, a positive history of group success, or external competition likely lead to strong group social identity (Gibson \& Early, 2007). In this context, a group with strong social identity would be one where all the members of a work group self-identify as 'we', and a weak group social identity would be one where some or all members default to ' $\mathrm{l}$ ', even when bound in a group setting.

Zohar (2000) studied group safety climate as a supplement to organizational safety climate. He found within group homogeneity and between group variation. Both of these findings would be predicted by social identity theory (Hogg \& Terry, 2000). Individuals tend to self-categorize on features that create a distinct identity that distinguishes one group from another (Ellemers, de Gilder, \& Haslam, 2004). However, Zohar (2000) attributed these inter-group variations to leadership differences, as opposed to a potential alternative interpretation of differences in coworker-generated safety values and norms. 
Proposition 2: Individuals working in safety critical or highly safety focussed groups will have high safety oriented social identity with the group.

The referent group implied in Proposition 2 is that of one's direct work group or partners and extends outward to include membership in one's profession (i.e. self-identify as a firefighter, river guide or paramedic).

\subsubsection{Internalizing coworker and group values}

The modern work on social identity theory is really a discussion regarding values adoption or internalization (Hitlin \& Piliavin, 2004; Hogg, 2000). If individuals choose to identify as ' $\mathrm{l}$ ' or ' we' depending on the situation, then certain values may be temporarily applied or situational. Values theorists would agree, and point out that the influence of individual values is in competition with normative pressures (Bardi \& Schwartz, 2003), as in values can be over-ridden by situational or social demands. But given the learned nature of values (Cable \& Parsons, 2001; Lauver \& Kristof-Brown, 2001), social identity may not be an 'either/or' scenario but instead a spectrum of value congruence with others.

Chiaburu and Harrison (2008) discuss how coworkers can influence individual opinion, attitudes and understanding of "how to internalize versus externalize core elements of one's job" (Chiaburu \& Harrison, 2008: 1085). Ashforth and Kriener (1999) discuss the same coworker effects in regards to individuals internalizing and externalizing aspects of jobs considered 'dirty work'. Internalizing was defined earlier as "taking in values, attitudes, or regulatory structures, such that the external regulation of a behaviour is transformed into an internal regulation and thus no longer requires the presence of an 
external contingency" (Gagne \& Deci, 2005: 334). Coworkers are looked to for cues about how to think and behave, and individuals use these cues to construct their own attitudes, interpretations, and meanings (Rosso, Dekas, \& Wrzesniewski, 2010). In this respect, coworker values become internalized and potentially adopted as one's own (Parks \& Guay, 2009). Regarding safety values, "when workers assign personal relevance to safety issues, it is expected that they will have strong attitudes about safety" (Fugas, Silva, \& Meliá, 2012: 471), where attitudes include values and beliefs.

Social-information processing literature offers an explanation for this. The basic premise of social-information processing theory (SIPT) is that individuals adapt their attitude, beliefs (and eventual behaviour) to their social context and to lessons learned in the past (Salancik \& Pfeffer, 1978). SIPT authors integrate a number of existing research streams to argue that "If coworkers continuously maintain that a job is horrible, boring or undesirable, the individual must either reject their judgments or assimilate them into his or her judgement" (Salancik \& Pfeffer, 1978: 229). Individuals are susceptible to coworker influence because coworker perspective can compensate for the individual's uncertainty or ambiguity in the task or job. Secondly, coworker influence may be accepted because "the worker may want to agree with coworkers, if only verbally, to fit in. These repeated verbal agreements may eventually convince the worker himself" (Salancik \& Pfeffer, 1978: 229).

McLain (2014) examined SIPT aspects of social referencing in relation to individual safety attitudes. The author found relationships between an individual's sensitivity to social 
information, the individual's social safety cognitions, and the individual's own safety values - in other words, social cues and one's understanding of the safety environment influenced individual safety beliefs (McLain, 2014).

When there is strong identification with a group, individuals are more likely to view group safety values as their own (Hogg \& Terry, 2000). This in turn influences individuals' propensity towards safety performance congruent with group outcomes. Selfdetermination theory (SDT) offers further explanation and a mechanism by which coworker or group values can become individual values. In effect, SDT moves group outcomes from an imposed goal or external form of motivation (i.e. norms) towards voluntarily internalized intrinsic motivation (Ellemers, de Gilder, \& Haslam, 2004). SDT represents a process by which group identity becomes incorporated into self-concept.

SDT explains how external influences become internalized. It does so by differentiating between autonomous motivation and controlled motivation (Gagne \& Deci, 2005). Autonomous motivation is wholly volitional and internalized; the activity aligns with the individual's self-concept and values, even though the activity is not inherently interesting (i.e. not inherently intrinsically motivating). Controlled motivation is external - applied pressure to act in a certain manner - that relies upon reward or punishment for compliance (Gagne \& Deci, 2005). Barker's (1993) 'concertive control' norms, for example, became a form of coworker-generated controlled motivation. These two ideas represent opposite ends of a spectrum of extrinsic motivation (Figure 6). 


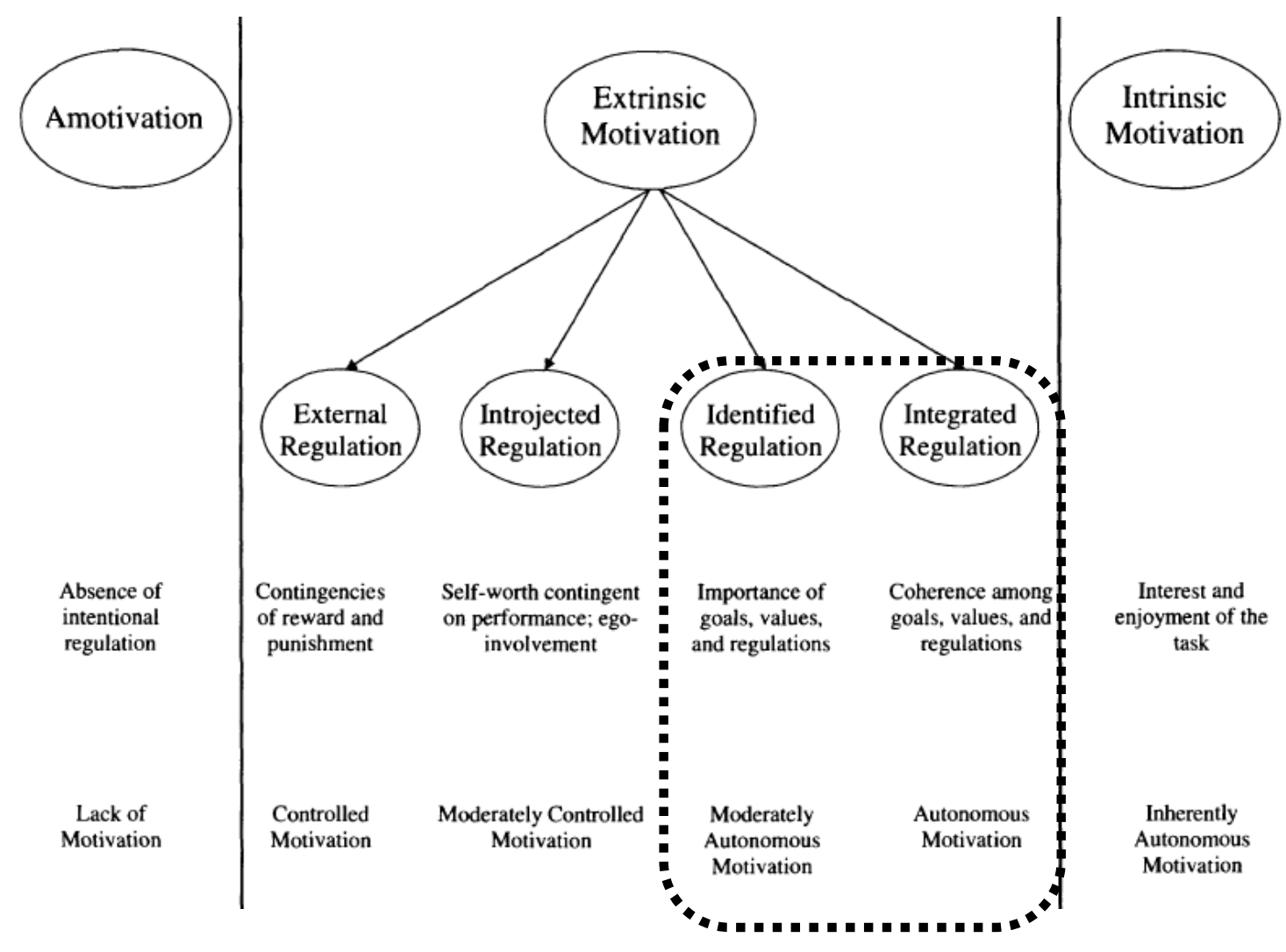

Figure 6: Extrinsic motivation spectrum, from Gagne and Deci, 2005

SDT becomes the link between self-concept and group identity theory with its focus on autonomous, extrinsic motivation. For both 'Identified' and 'Integrated' regulation (highlighted area, Figure 6), individuals are internally motivated based on alignment of goals and values between the task at hand and their own self-concept. The wearing of a safety harness could exemplify this. While not inherently interesting or enjoyable (intrinsically motivating) it does align with one's goals and values of self-preservation. Another example: disinfecting an operating room. While not intrinsically motivating, it aligns with the operation team's goals and values of caring for a patient's health. These tasks, according to SDT, would be autonomously completed (without need for reward or punishment) as long as they aligned with individual self-concept. Theoretically, a nurse is 
better motivated to disinfect an operating room than a minimum wage cleaner. Compared to an individual cleaner, nurses more readily internalize this type of activity based on self-concept and identifying with a group and its values.

Internalizing motivation can be promoted, according to SDT, when an individual has a sense of competence for the act, understands why and how the act relates to the larger context (relatedness), and has some choice and autonomy in execution (Gagne \& Deci, 2005). Most importantly for the model presented in this study, internalization is facilitated by "explicit or implicit endorsement of the behaviour by significant others" (Gagne \& Deci, 2005: 339). Coworker support for safety has been identified by several authors as an important dimension of perceptions of safety and safety climate (Törner, 2011; Zohar, 2000; Meliá, Mearns, Silva, \& Lima, 2008; Neal \& Griffin, 2006).

Proposition 3: Given a strong social identity, the individual is more likely to adopt and internalize coworker safety values rather than externally regulate safety values.

\subsubsection{Self-concept}

The ideology of self-concept suggests individuals strive to express and validate their behaviour, and that this is a fundamental human motivation (Shamir, 1991). From a safety performance perspective, this means that individuals will behave in ways consistent with their self-image. Risk takers will take risks, and conservative people will behave conservatively (Wilde, 1982). To this end, safety motivation is primarily regulated by internal values and self-monitoring (Bandura, 1989), yet the argument presented within these propositions points out how internal beliefs can adopt coworker and group values. 
Self-concept becomes modified by self-categorization and membership in a group (Hogg \& Terry, 2000; Dutton, Dukerich, \& Harquail, 1994).

Self-categorization theory believes that people voluntarily self-identify with a given social group (for example: a pilot, an athlete) and incorporate that identity into their selfconcept. Staying consistent with this self-concept becomes a need in and of itself, and a powerful trans-situational motivator (Shamir, 1991). Self-categorization plays a central role in determining which needs a given behaviour attempts to satisfy. Haslam and colleagues write: "we are motivated to live up to norms and to achieve goals that are relevant to our self-definition" (Haslam, Powell, \& Turner, 2000: 329).

Hogg argues this self-categorization mechanism is motivated by uncertainty reduction, as this is a "core human motivation" (Hogg, 2000: 224) tied to having control over one's life. Social categories are normative and so reduce uncertainty by prescribing behaviour, he argues. Several authors reference uncertainty reduction as a key social function of coworkers (Fugas, Melia, \& Silva, 2011; Fogarty \& Shaw, 2010; Chiaburu \& Harrison, 2008; Jiang, Yuc, Li, \& Li, 2010).

Individual motivation becomes maintaining self-concept and fulfilling expectations associated with a social category. The same logic could be extended to safety motivation - an individual's safety intentions will reflect safety values congruent with the social group and role or profession. Bunderson and Thompson (2009) write:

By identifying with the [workplace] community, individuals [...] come to embrace the beliefs and ideologies of that community as their own and can therefore draw 
on these beliefs and ideologies to assign both personal meaning and social significance to their work. (Bunderson \& Thompson, 2009: 39)

Mullen's (2004) qualitative safety study found pervasive social influence and social motivation to preserve one's self-concept. She concluded that "individuals who have the need to maintain a desirable image will be more likely to comply with the perceived subjective norms regarding safety in the organization" (Mullen, 2004: 282). Salancik and Pfeffer (1978) describe how once an individual commits to or internalizes a value or behaviour, they develop justification for that commitment.

Proposition 4: Given a strong social identity, the individual is more likely to incorporate group safety values into one's self-concept rather than externally regulated safety values.

Proposition 5: An individuals' safety values and motivation will be directed towards protecting one's self-concept and social identity.

\subsection{INTERVIEWS: RATIONALE}

The heart of this research project was oriented around interviewing individual workers and probing their perspectives regarding coworkers' influence upon their own internalization of safety beliefs and safety practices. This qualitative aspect of the research proved successful in providing contextually rich data from which to examine a group level phenomenon of coworker influence.

The majority of research in the safety literature and the much larger motivation field is survey based. Reliance upon this methodology ignores the "power of context" (Latham \& Pinder, 2005: 486) in understanding cultural and motivational processes. Guldenmund writes: 
As [currently] applied by safety researchers, [understanding work culture norms] is deprived of much of its depth and subtlety, and is morphed into a grab bag of behavioral and other visible characteristics, without reference to the meaning these characteristics might actually have... (Guldenmund, 2010: 1466)

The interviews in this research allowed for exploring the research question with much more depth than would have been gleaned by survey. Denison (1996) points to qualitative methods as a requirement if one is looking to appreciate the unique aspects of a specific social setting. From a safety perspective, Fugas, Silva, and Meliá (2012) studied mediating factors between climate, coworkers, and safety behaviour, but went on to recommend qualitative methods to enhance the understanding of such effects.

The purpose of these interviews was to understand aspects of peer influence - a nascent field of study. As discussed earlier, when working with nascent fields of study a researcher is expected to "suggest new connections among phenomena" (Edmondson \& McManus, 2007: 1158), therefore such fields may be best approached by open ended, qualitative inquiry. Edmondson and McManus (2007) elaborate that "because little is known, rich, detailed, and evocative data are needed to shed light on the phenomenon" (Edmondson \& McManus, 2007: 1162). In this case, interview methodology was selected as a means of maximizing realism of context (Scandura \& Williams, 2000), recognizing that it comes with some trade-offs in precision and generalizability (McGrath, 1981). As the most widely used methodology in field studies (Scandura \& Williams, 2000), the ability of interviews to provide rich, valid data stems from its orientation around the informant's context, using their language and perspective rather than relying upon the researcher's perspective or other secondary data (Myers, 2009). 


\subsection{INTERVIEWS: USE OF METHODOLOGY IN LITERATURE}

Interviews are used very little in either the safety or the motivation fields. Industrial safety research has its roots in engineering, hence has a strong quantitative and positivist bias. The occasional published article on safety performance is overshadowed by research on accident data in different sectors or the effects of organizational factors upon safety. These articles are almost exclusively survey based. Safety Science, for example, considered one of the leading journals in the field and the one most likely to deal with 'soft' safety such as culture, climate, and motivation (and notably less quantitative than Journal of Loss Prevention in the Process Industries, for example), published over 1000 articles in the past seven years. Approximately $12 \%$ of them used interviews (129) with the rest being survey based articles, data analysis (i.e. accident rates), and an occasional conceptual piece or case study. Of the interview papers, they almost exclusively used interviews as a pretest for a survey, with $\mathrm{N}$ ranging from 9 to 44 . There are, however, more interview papers appearing in the last two years than in the previous editions of the journal.

The few papers that relied exclusively upon interviews used the methodology in somewhat different manners. A study on a Norwegian oil platform, for example, used semi structured questions with two interviewers across a variety of workers and job types (Høivik, Moen, Mearns, \& Haukelid, 2009) asking about safety culture in the workplace (N =31). The paper explains the methodology and analysis with some detail, including the coding scheme. It also refers to previous work and principles upon which the interview 
questions were based, as well as methodological precedents or models which the authors followed (in this case Giorgi, 1985).

A second sample interview paper asked wildland firefighters to recount a specific event, looking for behavioural information exploring how individuals recognize an emerging crisis (Barton \& Sutcliffe, 2009). The authors' line of questioning basically asked '...And then what did you do?' to assemble a narrative, rather than probe attitude or rationale (Barton \& Sutcliffe, 2009: 1334). Interviews were purposively sampled ( $N=28$ ) in order to capture a wide variety of events, management agencies, and work positions. Each narrative created a mini-case study, which were then mapped and compared across-case (62 cases in total). From these, a theory of social processes emerged that disrupt "dysfunctional momentum" (Barton \& Sutcliffe, 2009: 1335) and allow for a crisis to be recognized as imminent.

Even in the much larger motivation field, surveys are largely the tool of preference, and researchers choose to canvas large populations rather than explore in-depth mechanisms. Motivation research cuts across disciplines, however this survey-based trend persists. In their comprehensive literature review of work motivation in nursing (1990 to 2009), Toode, Suominen \& Routasalo (2011) found only three studies that used qualitative interviews compared to 20 quantitative survey based studies and one quasiexperimental design. These three interview based studies relied upon $\mathrm{N}$ of 13, 14 and 16, and employed a semi-structured thematic analysis design. The interview studies appeared to leverage the potential benefits of the methodology and hinted at a deeper 
interpretive understanding, finding "attaining a sense of meaning" from the nursing work as a motivating factor and "finding value in a team oriented job" (Toode, Suominen, \& Routasalo, 2011). The survey based studies were limited to concrete qualities and traits of the job.

The above papers tended to describe their analysis in greater detail than their methods, with an emphasis on coding schemes. In this literature, the interview methodology is relatively taken for granted. Perhaps the deeper analysis procedure presented in interview safety and motivation research is due to these qualitative papers' inclusion in a largely survey based field. More care is taken to explain and defend analysis as rigorous a typical 'defensive approach' identified by McCracken (1988). There is no apparent trend to reference a single methodological source - there is a wide variety. Content analysis, however, is the typical analysis approach presenting found themes. The various analytical approaches cited all tend to conform to an interpretive multi-step process: review the whole transcript; code it by section; draw conclusions from coded data; summarize and present. This is the case even if the work in question is not actually interpretive but is testing hypothesis - a point of note given this research project's proposition guided research model.

There are two notable exceptions. Colley and Neal (2012) interviewed middle managers and workers regarding safety schema across authority levels $(N=25 ; 6$ managers, 7 supervisors, 12 operations workers). The interview methodology section is very thorough and directly references McCracken's (1988) long interview approach. The authors define 
'semi structured' and indicate the interviewer's role in prompting and guiding the interview (as opposed to directing it). The methodology section explains "Interview questions were designed to be unobtrusive to enable participants to tell their own story, on their own terms... questions were phrased in non-directed ways and were purposively broad and open ended" (Colley \& Neal, 2012: 1781). Their analysis section is atypical, in that it relies primarily upon large colour figures of a key concept map and a second of thematic clusters. This conveys the results convincingly, but is surprising given the quantitative journal in which it appears, and reflects either a break in tradition or a trend towards more open interpretation of qualitative reporting.

Mullen (2004) used interviews for investigating factors that influence unsafe behaviour at work. Her findings of pervasive social influence and social motivation to preserve one's image is relevant to this thesis, however her methodology, like Colley and Neal (2012) is explicit in its reliance upon McCracken's (1988) approach. While not as thorough as Colley and Neal (2012), the semi-structured interview protocol is outlined, as was the analysis procedure (utilizing grounded theory in this case). The findings are carefully structured with appropriate use of quotes to exemplify key themes. The significant critique against this paper is that it is based on only seven interviews.

The most significant improvement upon interview methodology as typically published in the safety and motivation field would be an explicit articulation of the interview approach. All but two articles simply state semi-structured interviews were used, leaving the reader to infer what (if any) philosophical approach grounded the work and what 
methodological tenets it adhered to. Perhaps it is the journal's practice to cut such parts of the methodology from the articles, but Colley and Neal (2012) (and to a lesser extent Mullen, 2004), with their full treatment of philosophy and procedure following McCracken's (1998) long interview, break the mold.

The same sampling of papers above often included (as an appendix) coding themes or data, but only Colley and Neal (2012) and Mullen (2004) included the actual interview questions. The inclusion of questions seems essential to assess the quality of the work and rationality of the conclusions. The interview questions are what attach the research aims to the final claims.

The Colley and Neal (2012) approach becomes a model to follow: context, philosophy and mechanics of method, analysis, and conclusions. The logic can be followed from beginning to end. This work did not include many quotes, instead using tables for the main themes with one or two exemplary quotes. This makes the article highly readable as the text is the researcher's logic as opposed to the informant's extracted words. 


\section{METHODOLOGY}

This research utilized proposition-guided interviews to examine how coworkers influence the internalization of safety. The unit of study was at the individual level of analysis. The phenomenon under study was the mechanisms of influence that coworkers have upon the individual internalizing safety. The heart of this research project was oriented around interviewing individual workers and probing what influence coworkers have regarding individual adoption of safety principles. Information gleaned at the individual level informed the group level investigation of coworker influence. The qualitative nature of the project provided contextually rich data allowing examination of peer influence and the internalization of safety.

\subsection{INTERVIEWS: SEMI STRUCTURED APPROACH}

The interview strategy closely aligned with McCracken's (1988) long interview approach. Published research tends not to articulate the semi structured interview approach it prescribes to (discussed previously), and McCracken's (1988) is one of many available.

McCracken's (1988) strategy uses open ended questions and prompts to let the informant tell their own story. McCracken claims this method is ideal to glimpse an individual's logic, mental models, shared assumptions, and cultural categories - all of which are concepts pursued by this research. He differentiates his approach from active and/or unstructured interviews very clearly, warning interviewers to "manufacture distance" in both the interview itself (by avoiding active listening, for example), and in the data analysis stages (McCracken, 1988: 21). Rather than read into what the informant says, the interviewer 
asks clarifying questions to force articulation of assumptions. This is very different than Holstein and Gubrium's (1995) active interview, for example, which is based on the philosophy "that all interviews are reality-constructing, meaning-making occasions, whether recognized or not" (Holstein \& Gubrium, 1995: 4). While the social construction of interviews is recognized, this research followed McCracken's structure in that it 'controls' for the interviewer. Using McCracken's distanced approach also aligns better with the safety fields' primarily quantitative, survey based, history and culture.

For this research, the ultimate purpose of the interviews was to understand the influence coworkers have upon the individuals' internalization of safety. As each informant has a unique perspective, McCracken's long interview approach allowed for divergent avenues to be pursued. A guiding set of questions ensured the interviews covered the same terrain and "conversational context"; the "direction and scope of discourse" was dictated by the interviewer, but the content was provided by the informant (McCracken, 1998: 24). As the adoption of group safety beliefs were under discussion, questions followed the leads opened up by the informants.

\subsection{SAMPLE SELECTION}

The sampling frame for this research was a selection of workers from safety critical or 'process safety' industries: firefighters, paramedics, and whitewater raft guides. Workers for whom safety is their primary work task are considered to be in 'process safety', whereby "the risks and uncertainties to be managed are directly linked to the primary work task of the organization [and] potential damages result from failures in the 
execution of [...] this task" (Grote, 2012: 1984). Those involved in process safety industries have a core task of producing safety (these are also known as safety critical industries). Unlike personal or occupational work safety, where damages typically apply to the worker involved, process safety errors may jeopardize the safety of many individuals beyond the employee. Firefighters, for example, put their own, their coworkers' and the public at risk with unsafe behaviour. Likewise, with paramedics who can harm their patients and whitewater guides who can injure their clients. These sectors share the core task of producing safety and having a duty of care towards their 'clients', whether it be the public, a patient, or a tourist.

Most safety studies' sampling frames do not differentiate between process and personal safety. The distinction between process and personal safety is recognized as important as "causal mechanisms involved in promoting personal versus process safety most likely differ" (Grote, 2012: 1985). Some of these differences are hypothesized to be differences in the visibility and complexity of risks (with process safety typically displaying more involved safety interactions), required competence for producing safety, inherent feedback mechanisms for unsafe behaviour, and conflicts between safety and production demands. Intuitively, process safety workers, or those immersed in safety critical activities, will have safety top-of-mind. The presence of safety as the core work task makes these workers valuable informants in discussing safety and in discussing how coworkers influence the internalization of individual safety. 
As coworker influence is the focus of this research project, safety critical work environments that rely upon a team or partnership arrangement were targeted. Firefighters are tasked to a dedicated crew for years at a stretch. Likewise, river guides work for small, seasonal organizations with a strong social group that work and live together. Paramedics work in teams of two.

The role that safety plays in any one individual's job varies across domains. For this reason, three different process safety or safety critical industrial sectors were examined, in an effort to triangulate the different influences coworkers may have upon internalizing safety.

\subsection{SOLICITING PARTICIPATION}

Research proposals were submitted to, and accepted by, a mid-sized urban fire service, a rural paramedic service, and a small whitewater rafting adventure outfitter.

Via the Fire Service Training Officer, a random selection of city fire stations were identified that represented a range of operating features: high call volume stations and low volume stations; and technical rescue stations and basic response stations. From this random variety, the Training Officer solicited volunteer crews via their crew captain, based on a script provided by the researcher (included in Appendix 1). Many stations volunteered, allowing the researcher to randomly select five stations and crews to interview. A total of 28 individuals agreed to participate. This represents five full crews, as no individuals declined participation. 
The rural paramedic service Deputy Chief arranged to have the researcher visit two dispatch bases. An email announcement regarding the research was sent to the base in advance. The researcher verbally solicited participants on site over the course of the work day (as workers came through the base). 11 individuals agreed to participate and two declined participation.

The whitewater rafting company, via the Operations Manager, distributed an email invitation to their whitewater guide staff, soliciting participation in the study. Volunteers responded directly to the researcher and arrangements were made to meet on site after work hours. Six individuals volunteered. Further whitewater guides were interviewed beyond the host company, based on a snowball sampling technique leveraging the original volunteers. A further 10 guides represented several different employers and geographic operating areas.

\subsection{SAMPLE DESCRIPTION}

A total of 55 individual interviews were conducted, distributed across firefighting, paramedic and whitewater guiding sectors. Firefighting provided 28 volunteers, paramedics 11, and guiding 16 (Table 1). 18\% of the informants were female - low by societal demographics but representative of these male-dominated sectors. The paramedic and guiding sectors' female representation was typical for those workplaces ( $36 \%$ and $25 \%$ respectively), however the firefighting representation was significantly higher. The urban fire service has approximately 1000 service members, 30 of whom are 
women (3\% of the workforce). In this study's sample, female firefighters represented $7 \%$ of that sector, even with only two female informants.

Table 1 Demographic distribution of sample

\begin{tabular}{|c|c|c|c|c|}
\hline & Total & Firefighters & Paramedics & Guides \\
\hline Individuals & 55 & 28 & 11 & 16 \\
\hline Male & 45 & 26 & 7 & 12 \\
\hline Female & 10 & 2 & 4 & 4 \\
\hline \multicolumn{5}{|c|}{ Years' Experience } \\
\hline $1 y r$ to 5 & 19 & 6 & 4 & 9 \\
\hline $6 y r$ to 10 & 17 & 8 & 3 & 6 \\
\hline $11 \mathrm{yr}$ to 15 & 5 & 3 & 1 & 1 \\
\hline $16 y r$ to 20 & 4 & 3 & 1 & 0 \\
\hline $20+$ & 10 & 8 & 2 & 0 \\
\hline
\end{tabular}

The age of the informants ranged from 20 years old to late 50 s. The sample individuals' work experience in their sector was typically below 10 years (Table 1 ). Firefighting and paramedics both had representation of long time workers, with a number having over 20 years of experience. The years of experience in the guiding sector is representative of the younger age of the workers in that field (typically 20-30 years old), with no representative having over 15 years of work experience.

Inclusion criteria for this study was front line, non-managerial roles that directly produce the safety outcomes expected of these and other safety critical industries. Within these criteria, the sample included some variance in levels of authority. Of the 28 firefighters, seven were senior crew leadership positions of captain or lieutenant. Among the remainder, a formal hierarchy exists at the crew level of 'senior man' down to the rookie (the rookie being the youngest on the crew, even if having been with that crew for several years). A fire crew consists of either four or seven firefighters, one of which would rank 
as captain. It is not uncommon for firefighters to spend their whole working career in this field, working up the ranks to eventually lead a crew. This fact is reflected in the higher years of work experience compared to the other sectors in this study.

For the paramedic field, eight of the volunteers were primary care paramedics (PCP) and the remaining three were advanced care paramedics (ACP). ACPs have three years of education (versus PCPs two years) and are licensed to provide a higher medical care standard. Paramedics work in teams of two. When paired with a PCP, the ACP typically takes the lead attending role, however from a formal authority perspective both paramedics in a team are considered equals even with the differential in qualifications and medical standard expected. $27 \%$ of this sample were ACP qualified, close to the service's approximate breakdown of $35 \%$ ACP and 65\% PCP. In this sample, four of the paramedics were full-time and the remainder were casual, reflecting the sector's move away from full-time employment with benefits, and towards casual or contract positions. Typically, only the full-time paramedics are paired to a consistent partner; part-time paramedics are tasked to a new partner every shift, often another casual employee.

The whitewater guide sample for this study represents seven trip leaders and nine basic guides. A whitewater raft trip would range from two to as many as eight guides - each responsible for the navigation and care of one boat of clients. A trip leader, also a guide, would be responsible for the whole trip's function. The trip leader designation may be a temporary or daily rotation while for another company the trip leader may be a formal leadership role earned with seniority or higher qualifications. Five of the seven trip 
leaders in this study had over six years of experience, representing the senior workers in this sector's sample. For many workers in this field, guiding is a temporary stop on a career path, evidenced by the relatively low average years of experience.

The individuals in leadership roles in each of the sectors studied are included in this study for two reasons: the individuals fit the inclusion criteria of front line roles that directly produce safety outcomes, as their leadership functions are operational rather than managerial in nature; and these leader roles are considered by the remaining workers and the fields in general to be an important element of the social fabric of the work teams, as the leaders are considered 'one of them' rather than removed from the team.

\subsection{DATA COLLECTION}

This research called for interviewing workers in safety critical industries to examine the influence that coworkers have upon individuals internalizing safety beliefs. The research proposal also called for at least one group interview per sector to further examine social constructs of norms and safety culture. Approval from the Ethics Review Board was obtained.

Data collection and the interviews took place between April and June of 2016. The urban fire service arranged for the researcher to visit the selected stations during work hours. Interviews were conducted in a private office at the individuals' convenience, back to back as the work day allowed. Occasionally an interview would be interrupted for a fire alarm or call, and would continue after the crew's return. A total of five station visits accumulated the fire sector data. The rural paramedic service interviews occurred during 
work hours, timed to coincide with shift changes, taking place in a private lounge area at their base facility. Three visits were completed. The whitewater guides were interviewed in the early evening after their work day, in a secluded outdoor picnic table area at the host location, on two different occasions. Further guides were interviewed either over the phone or at the researcher's work office on various dates.

The semi-structured interviews typically lasted 20 to 40 minutes. Follow up or elaboration questions went beyond the scripted questions to elicit examples, ask for explanation of assumptions, or to follow leads presented by the informants, as endorsed by Miles and colleagues (2014) and McCracken (1988). The full interview protocol is outlined in Appendix 2 and the interview questions listed in Appendix 3. All interviews were audio recorded on a Zoom $\mathrm{H} 1$ digital recorder. Written notes were taken in addition to the audio files. Interview recordings were transcribed by a third-party service provider into a Word text file. Anonymity and privacy were addressed by numbering the audio files chronologically and with no associating personal names or identifiers. Any names of coworkers that emerged during interviews were removed from the transcription files. The digital audio files will be destroyed upon completion of this project, and transcripts and notes will be secured in the researcher's work office for potential publication in the future.

Six group interviews were conducted: one for each of the fire crews interviewed (four to seven participants per group, five groups total), and one at the rafting company (four participants). The participants in the group interviews were subsequently individually 
interviewed. No group interview could be arranged with the paramedic sector. Group interviews lasted 30 to 40 minutes. Care was taken in coding to attribute utterances and statements to the different individuals present. The group interview questions are included in Appendix 3.

Lastly, observations of the physical work places were noted by the researcher and recorded as hand written notes to complement the associated interviews. In particular, overt or subtle safety messaging was noted, such as occupational health and safety information, pictures or posters, wall maps and posted instructions, and the general tone of the setting with regards to safety. The researcher also participated in one ride along to a fire call, notes from which contributed to that crew's interview data, and had first-hand knowledge of the rafting operation based on previous consulting work. This operational data informed the results discussion.

\subsection{DATA ANALYSIS}

McCracken's (1988) long interview approach is designed to examine the development of social categories. His preparation stages - the literature review and interview design - is a means of pre-analyses of the topic under study. For the purposes of this research project, the pre-analysis stages led to the development of theory driven propositions that structured the subsequent interviews. The interviews themselves generated the data by which to examine the phenomena under study: the influence of coworkers upon the individual internalization of safety beliefs. These guiding propositions also formed the initial categories by which the interview transcripts were analysed. 


\subsubsection{Content analysis}

The interview data was analysed via content analysis. Content analysis is a loosely connected group of qualitative analysis methods that are ideal for seeking structures and patterned regularities within a data set and identifying symbolic content (Singleton \& Straits, 2010; Myers, 2009). Content analysis does this by, and what various content analysis methods have in common is, assigning categories and coding data into these categories.

Miles, Huberman and Saldana's (2014) three stage qualitative analysis method relies upon content analysis. Miles and colleagues (2014) recommend data analysis starts before data collection, with categories informed by the research question. These categories form the basis of data reduction, but are modified as coding patterns emerge from transcript data. At this early stage, patterns in informants' response and linkages between ideas start to emerge (Miles, Huberman, \& Saldana, 2014).

Miles, Huberman and Saldana's (2014) second step is to organize the data into some presentable format to address the research question. This might include narrative samples, causal maps, or displays of response categories. The intention is to connect the data to the research goals. Relationships among variables and key concepts that emerged in coding are presented in order to set the foundation for the findings that follow. The last step is to analyze the presented data. Conclusions are drawn from a systematic analysis of the results and can be conceptualized into a working model. 
McCracken's (1988) content analysis approach is similar but aligns with his long interview agenda and philosophical underpinnings of distance. He further breaks data reduction down to examining "utterances", which combine to form "observations". Going beyond the transcript now, these observations are analyzed against each other as themes, from which inter-relationships and generalizations are made. McCracken's (1988) last stage of analysis is going back to the beginning to compare what was found against what was expected. He characterizes this step as moving from the world as the informants see it (via utterances, observations, and themes) to how the researcher now sees it - from what was said to what is now known (McCracken, 1988).

\subsubsection{Initial coding and categories}

The interview transcripts were examined line by line identifying and isolating what McCracken (1988) referred to as "utterances". These utterances as lines of text were coded and categorized using NVivo software. The propositions guiding the study were the initial categories into which the data was organized, and formed the first cycle coding nodes. For example, 'Safety norms as representative of agreed upon safety values' (Proposition 1) was an initial coding category, as was 'Safety in relation to self-concept' (Proposition 4). Miles, Huberman and Saldana (2014) name this approach Hypothesis Coding, where codes are drawn from theory or prediction of what will appear in the data before it is collected. For this study, under used (in safety science) social theories were the basis upon which the propositions and coding were drawn. Using this method, the first cycle of coding was deductive in nature, moving from a broad theoretical starting point to specific observations drawn from the informants' own words. Where applicable, 
any single utterance was coded into multiple categories. Appendix 4 lists the first cycle coding categories.

\subsubsection{Analysis and second cycle coding}

During initial coding of each interview transcript, notes and researcher observations were captured in memo form. These memos were cross referenced to the hand-written notes taken at the time of interviewing, and any insights captured in the memo format. The informant's commentary was further analysed and matrix plotted against the guiding propositions and a number of related variables that comprise the social theories informing this study. For example, SDT informed Proposition 3: Given a strong social identity, the individual is more likely to adopt and internalize coworker safety values. SDT further relies upon volition, identified regulation and integrated regulation as aspects of this internalization. If the informants' interview discussed, alluded to, or provided examples of these variables they were noted in the analysis matrix. With all 55 interviews

plotted, the matrix displayed an overall view of level of support or presence regarding any one proposition or variable.

Next, the contents of the first cycle nodes were analysed and further refined into second cycle codes. These second cycle codes emerged as themes or common sentiments from the group of interviews. For example, the first cycle category 'Aspects of coworker influence' was further organized into 'Ambiguity reduction', 'Need to fit in', 'Social support for safety', 'We vs. I', and 'On own - no team'. Each of these second cycle categories emerged inductively from the utterances provided by the informants. These 
second cycle codes informed the findings and theory building to follow. Appendix 4 lists the second cycle coding categories. During this second cycle coding, theoretical insights and observations were captured in memo form. While propositions initially structured this research, the data itself started to direct the analysis and interpretation. Eisenhardt (1989) recognized this as one of the key aspects of qualitative analysis - moving from what was said to what the researcher finds. A series of NVIVO analysis queries were performed on the first and second cycle nodes, as both a means of understanding the data and to create visual representations of the emergent findings. These latter analysis steps evolved from data manipulation to meaningfully addressing the research question.

\subsubsection{Interpretation of findings and validity of conclusions}

Each individual study presents its own demands as to how the findings are arrived upon, organized, and presented. McCracken (1988) explains that "the exact manner in which the investigator will travel the path from data to observations, conclusions, and scholarly assertion cannot and should not be fully specified in advance" (McCracken, 1988: 41). Some methods of content analysis proceed from coding directly to quantification or developing some enumeration system (for example, comparing frequency counts between categories), while other methods are more closely aligned with grounded theory (Singleton \& Straits, 2010).

As this study ascribed most closely to McCracken's (1998) long interview approach, he provides some direction in the interpretation of data. The goal is to read through the utterances into the assumptions and beliefs "from which it springs" (McCracken, 1988: 
44). These become the researcher's observations, and the various observations are compared back to the transcripts and to other observations. Observations are assembled into themes. Whether these themes are presented as hierarchical, cyclical, or concurrent is dictated by the context and the researcher's judgement. This is where the data is left behind and findings are established. The research material itself dictates a suitable path towards addressing the research question.

The analysis stage, like all qualitative research, relies upon the researcher to apply distance and rigor to the data. McCracken (1988) is upfront about the role the researcher plays in the interpretation role, and relies upon thorough preparation to aid in data analysis. He explains the whole process:

The object of analysis is to determine the categories, relationships, and assumptions that inform the respondent's view of the world in general and the topic in particular. The investigator comes to this undertaking with a sense of what the literature says ought to be there, a sense of how the topic at issue is constituted in his or her own experience, and a glancing sense of what took place in the interview itself. The investigator must be prepared to use all of this material as a guide to what exists there, but he or she must also be prepared to ignore all of this material to see what none of it anticipates. If the full powers of discovery inherent in the qualitative interview are to be fully exploited, the investigator must be prepared to glimpse and systematically reconstruct a view of the world that bears no relation to his or her own view or the one evident in the literature. (McCracken, 1988: 42)

Ultimately, every research project is judged against its own merits. For this reason, the whole of the project must be carefully thought out with clear logic links between all steps and assertions (Myers, 2009). In this manner, the research method becomes secondary to the researcher (Spiggle, 1994) and one's ability to assemble a rational argument given 
the collected data. There are guidelines to support this. Myers (2009) outlines these as 'Principles of Interpretive Research', and includes (among others) principles of contextualization, hermeneutic cycle, dialogical reasoning, and abstraction. These, in effect, become logic tests of the work and its conclusions. McCracken (1988) presents a less ideological test, and asserts qualitative research must be exact, consistent, unified in its approach, and insightful. Eisenhardt (1989) summarizes assessing qualitative research as this:

When a [proposed] relationship is supported, the qualitative data often provides a good understanding of the dynamics underlying the relationship, that is, the 'why' of what is happening. (Eisenhardt, 1989: 542)

This research project may be assessed as valid if and when the following methodological conditions are met:

- The research rationale and intentions are logically presented

- The methodology aligns with the subject under study and is appropriately articulated to expose the mechanics of the research process

- The literature review and pre-examination of the subject under study points to analytic and socio-cultural categories; these inform the interview design, as per McCracken (1988)

- There is evidence of care taken to interview/collect data in a professional and consistent manner, as per McCracken (1988) 
- Data is analyzed and presented in a rational manner; there is evidence of contextualization, the hermeneutic cycle, dialogical reasoning, and abstraction as per Myers (2009)

- Conclusions are visibly drawn from the data and are exact, consistent, unified in their findings, and insightful as per McCracken (1988)

- The work (taken in its entirety) makes a contribution to knowledge and suggests why a phenomenon is evident.

The research methodology, as executed in this study, provided an opportunity to assemble and examine data with which to address the research question. What follows is the study's findings assembled into a logical line of reasoning. These findings, it is expected, will contribute to the emerging field of study of coworker influence. 


\section{RESULTS}

What is the influence of coworkers upon the individual internalization of safety? From the interview data, a number of plausible perspectives or themes emerged to answer this question. As this research project was proposition guided, some of the themes were targeted in advance and were expected to be present. More interestingly, a small number of unanticipated themes emerged, offering a perspective on coworker influence that provided insight beyond the intended reach of this project.

In order to present the detailed interview data, the expected and emergent themes, and to link these to the research question, a structured mix of narrative and data display is utilized. For each theme, a representative selection of what McCracken (1988) calls 'utterances' exemplify the specific responses, but collectively create the researcher's observations and findings. McCracken explains this technique as using the "particulars" to explain the "general properties" of thought (McCracken, 1988: 46). From this, a narrative emerges linking the data to mechanisms of coworker influence and to the internalization of safety. These phenomena are ultimately tied to theoretical principles and consolidated into this study's contribution to theory and practice.

The themes, when organized into a timeline, provide a narrative and represent a pathway through which coworkers influenced the individual's internalization or adoption of safety priorities established by their group. The themes can be separated into coworker or social influence themes, and individual internalization themes. A third category, trust, emerged as a pervasive lever enabling coworker influence and the internalization of safety. This 
was unexpected, as the idea of trust in relation to safety has seen very little exploration in the safety literature. Table 2 organizes the themes around which this results section is organized.

\begin{tabular}{|c|c|c|}
\hline Theme & Source & Category \\
\hline 1. Shared experience & Emergent & \multirow{4}{*}{$\begin{array}{l}\text { Social influence } \\
\text { themes }\end{array}$} \\
\hline 2. Norms as performance standards & Proposition 1 & \\
\hline $\begin{array}{l}\text { 3. Sensemaking and ambiguity } \\
\text { reduction }\end{array}$ & Emergent & \\
\hline 4. Social identity with group & Proposition 2 & \\
\hline 5. Internalizing safety & Proposition 3 & \multirow{2}{*}{$\begin{array}{l}\text { Individual } \\
\text { internalization } \\
\text { themes }\end{array}$} \\
\hline $\begin{array}{l}\text { 6. Self-concept with regards to } \\
\text { safety }\end{array}$ & Proposition 4 \& 5 & \\
\hline 7. Trust & Emergent & Pervasive \\
\hline
\end{tabular}

Shared experience (Theme 1) formed the basis of interaction upon which a group negotiated its own norms. These norms were articulated by informants as 'performance standards', but interview commentary and field observation both pointed to these norms as socially enforced safety behaviour expectations (Theme 2). 'Buying into' these safety standards or meeting these performance expectations enabled a level of social identity within the group, and an avenue for individuals to self-identify with their work group (Theme 4). The shared experience theme also formed the basis for group level sensemaking with regards to safety (or more specifically with regards to conflict regarding safety, policy, and getting the job done - Theme 3). Discussion of the internalization of safety (at the individual level, Theme 5) referred to sensemaking plus the aspect of socially identifying and 'buying in' to the group's priorities. Further, this internalization influenced (for most individuals in this study) an aspect of their self-concept with regards to safety 
and their ability to perform (Theme 6). Unexpectedly, trust emerged as a theme that was discussed at several stages in the pathway assembled above (Theme 7). Each of these themes - expected and emergent - are examined in detail below.

Three different safety critical sectors were examined for this study. Two of the three, firefighters and whitewater guides, recognized coworker influence on a variety of levels and generally showed conclusive support for the thematic pathway identified above and for the propositions that guided the research. The third sector, paramedics, showed varied support for the different propositions and for the role coworkers may play in influencing individual safety. The differences that paramedics presented offer an alternative perspective of the role and limitations of coworker influence regarding safety. All three sectors will be discussed in relation to their support for the themes introduced above.

Further, the findings below are compared to the theoretical variables that initially anchored the guiding propositions. The theoretical implications of these findings are detailed in the discussion section.

\subsection{REPORTING RATIONALE}

While a qualitative study focuses on the qualities of a phenomenon, to make sound generalizations some measure of consistency and representativeness can be presented by showing how much a phenomenon occurs in the data. Miles and colleagues (2014: 282) refer to this simply as 'counting' qualitative data, and suggest it is best used to verify hypothesis or propositions, to summarize large batches of data, and as an objective 
analysis tool to avoid bias - all useful for the present reporting structure. Given the large amount of data generated by 55 interviews, data display tables summarize the responses per theme and per proposition, however to preserve the narrative flow, these display tables are collected into a dedicated section that follows these results. Sample size is indicated by $\mathrm{N}$ representing the full sample, and $\mathrm{n}$ is used when considering sub groups from the full sample. Select quantitative data are also included as footnotes in the narrative to provide specific support values for a given theme, proposition or variable.

An informant need not have explicitly stated endorsement of a theme or proposition in order to prove it supportable. If the interview discussed, alluded to, or provided examples of the phenomenon under study this was noted in the analysis matrix as support for or against the theme. In this way, generalized support or rejection for a particular proposition was established, with a clear means of assessing the strength of endorsement. This is shown in the data display tables that follow the results narrative. The data displays provide an overall view in support of what the results narrative explains in detail. The results narrative ultimately becomes the basis for answering the research question: what is the influence of coworkers upon the individual internalization of safety?

\subsection{THEME 1: SHARED EXPERIENCE}

All the workplaces under study involved shared experiences. Working in a team or with a partner was a defining feature of the sectors targeted for this study. Since the shared workplace is a given, as a theme or collection of observations it is the qualities and the character of the shared work experiences that differed between sectors. The physical 
work locations, the observed social interactions between the coworkers, and commentary from the informants themselves shaped the foundation role that shared experience plays in coworker influence upon safety.

\subsubsection{Observation of the workplaces with regards to safety}

Schein (1985), in his early and influential explanation of organizational culture, anchored his model in shared group experience. One of the products of this shared experience is what he called 'artefacts and creations' of group culture; these include workplace policies and practices but also the function and feel of the physical workplace itself. The workplace, as an artefact or creation of culture, should provide cues to recognize the character of the culture it houses.

A total of six workplaces were visited in the course of interviewing 55 informants: one whitewater rafting resort, two paramedic bases, and three fire stations. The three sectors' work spaces were very different in function and feel. The whitewater rafting facility was the least formal (with regards to safety), the fire stations can be characterized as in a state of readiness, and the paramedic bases seemed more parking garage than workplace.

\subsubsection{Whitewater rafting workplace}

From a safety standpoint and as a work setting the whitewater rafting resort was the least formal. The facility hosts tourists and vacationers in a mostly open air setting. The main building housed an office, a check-in desk, and a large commercial kitchen; all other facility space was outdoor deck or covered balcony, oriented to focus attention on the 
riverfront view. Staff live on site in rustic cabins but shared communal space with vacationing tourists. 18 guides work for this company.

From a safety perspective, safety messaging was present but clearly targeted towards the clients. The check in area had several safety related poster boards serving as risk management/informed consent signage typical of this sector. The staff room contained no safety messaging (its walls were covered in old river posters and photos of raft trips past and present). The office area walls had maps, thumb tacked memos and historical aerial photos of the river. No employee occupational health and safety information was visible (which is a requirement under Ontario's Occupational Health and Safety Act, RSO 1990, c 0.1). The owner of the facility was present and managed daily operations. His presence was indicated by informants as the main vehicle to uphold service quality standards, and the trip leaders pointed to the owner as providing clear expectations regarding safety and risk tolerance on the whitewater trips.

The place of work for the guides is on the river. A senior trip leader delivers the key safety briefing to clients in the morning, and then all board a bus which drives to the top of the rapids. The guides organize their groups, climb into their rafts, provide safety instructions, and then run the rapids back to their resort facility. Each guide is responsible for the 10 passengers in their boat, as well as providing safety cover for the other rafts on their trip, all while moving continuously downstream. Their whitewater raft trip is significantly more formal than their base setting, despite it being a completely natural environment. The guides wore matching helmets and rescue life jackets, used whistle and hand signals to 
communicate in the rapids, the rafts traveled in a 'tight' line, and a focussed yet friendly tone was exhibited by the guides. Raft guides have a paradoxical role: take clients to potentially harmful places and then protect their safety when they get there. In other words, take untrained people into rapids to have 'fun', but not so much fun that it becomes dangerous. Overall, the safety priorities of this group of guides was visible in their on-water actions but not in their workplace facility.

\subsubsection{Paramedic bases}

Two paramedic bases were visited. One was the main hub station for the county and housed three ambulance crews per shift; the other was a secondary station that housed two crews. Both facilities were similar in layout and cinder-block feel. Paramedics spent most of their work day in their vehicle with a partner. When not attending a call, they were often transferring patients between hospital facilities or were directed to park on standby at certain locations to cover regions where calls were active. The base functioned primarily as a restocking point amidst other shift duties.

The base building itself was utilitarian: cinder block construction, heavy steel doors, security locks on every access point, and storage shelves lined with supplies and boxes. It was not designed to be seen by the public. The large unheated parking bay was the central feature where vehicles were re-stocked and cleaned. The majority of workers' time at base was spent here. Through a door was a 'control room' with desks and four computer stations lining the outside walls. Paramedics filed their reports here and received

emergency call directions (also sent to a screen in the ambulance). Beyond the control 
room was a small kitchen and lounge with a television. The place had the feel of a facility visited by many without any one person in charge - things were mostly neat and tidy but not quite. There was a deserted feel, as small jobs were left undone and abandoned when a call came in and workers left. The facility was very quiet and went long stretches with no one present. The dispatch radio was on continuously in the background, allowing one to overhear the continual exchanges between dispatch and ambulances on the road. A shift supervisor was likewise continuously on the road. The ambulance crews worked under the direction of dispatch, and only contacted a supervisor by phone when extra support was needed. There were no meeting tables or face-to-face seating. The control room had paramedics seated facing the wall with backs to each other. Even the kitchen had a bar-style counter with just one or two chairs. The interview data collected at these locations reflected the individualized nature of the work environment. Interviews with paramedics took place in the kitchen lounge area of each base, sitting shoulder to shoulder at the counter.

The control room and hallway walls were covered in technical safety briefing memos most as indistinguishable printed email reminders of procedure changes or notes on equipment use. Binders lined the desks containing Ministry of Health guidelines. An Occupational Health and Safety (OHS) board was prominent in the hallway, although it contained mostly union grievance updates relative to OHS claims. (The role of the union was rarely discussed in the following interviews - only one paramedic made reference to it; most paramedics are part-time, non-unionized, so the union applies to only a portion of the workforce). The safety material posted was best described as technical details, not 
in any way indicating big picture priorities. All entry ways and steps had anti-slip safety tape on the floor complying with OHS requirements, yet in the parking bay vehicles were parked in water puddles while plugged in to recharge, creating a shock hazard. One informant described his workplace:

People talk about security here, they talk about safety here but I see a lot of show as opposed to go [i.e. actual practice].

Paramedic

As a specific example of workplace safety, another informant pointed out the wall mounted exhaust meter in the parking bay, calibrated to measure accumulated gasoline combustion fumes. The ambulances run on diesel, rendering the meters useless.

\subsubsection{Fire stations}

Three different fire stations were visited for group and individual interviews. Any one fire station was home to three different crews who work in shifts, in order to have complete coverage 365 days a year. Two of the three stations were visited twice (visiting a different shift each visit), and the remainder once (for a total of five different crew visits). A crew consists of four or seven firefighters, depending on if the station housed one or two trucks. There was a wide variation in call volume among the stations visited. The downtown station responded to 15 calls per shift (a 24-hour period) while the crew in the modern suburbs responded to 20 calls per month. Only a fraction of the calls were fires. Most calls were for motor vehicle accidents or backup for medical emergencies.

Firefighters work 24-hour shifts, so cook, eat and sleep with their coworkers at the station, generating multiple 'family atmosphere' comments that emerged during the 
interviews. A large bay area housed the one or two fire trucks and all of the related PPE and equipment. An adjoining control room looked onto the bay and housed a bank of 1960s era switches and lights, retrofitted with digital screens showing maps and scrolling text of call information. Beyond these work areas was a large, commercial grade kitchen attached to a common room with a large dining table and comfortable couches. Separate sleeping rooms were down a hall, as was a communal 'training room' which was equally comfortable but was lined with whiteboards, books, and a television used for training lessons. Each station had an exercise room. Group interviews usually took place around the dining table or in the training room. Individual interviews took place privately in the training room or an office.

At a busy station, there was continual movement in and out responding to calls or cleaning up. At a slow station there was much down time. Regardless, both had in common an ethic and air of readiness. The parking bay and control rooms were spotless. PPE was at the ready - literally: fire pants were draped around safety boots that were open and ready. One step gets into both boots and pants. These were positioned at the step of the truck, where the door was already ajar awaiting to be jumped into. The researcher was present when the fire alarm sounded on several different occasions; the whole crew would be out the door, sirens wailing, in 20 seconds. The researcher was invited to accompany a crew on a fire call, riding in the truck with the firefighters and observing from a distance the tactics to deal with a house fire. Observations from this ride along are distributed throughout the narrative below. 
From a safety standpoint, the fire stations uniformly exhibited an air of preparation. Firefighters were constantly tinkering with something on the truck or equipment. The interviews sometimes referred to the need to keep busy so to not get lazy and lose the sense of readiness. Safety messaging varied slightly per station, with an overall trend of minimal presence. While one station had in the common room two 'stay safe' type posters, the others did not. One station displayed a series of framed 'glory' photos, including September 11 images. Large district street maps took up prominent space in the hallway and training rooms of all stations. Likewise, two of the three stations had prominent OHS boards in the training room, well organized with file folders. Several informants pointed to the OHS board during their interview in reference to standard operating procedures. The third station's OHS board was posted in the hallway, less prominent and not as organized as the other stations. These slight differences in work environment did not manifest itself in the individual interview data in any noticeable way. Firefighters in this studied worked in a unionized environment. The role of the union was not visibly present in the workplace, and only in one interview with firefighters did the role of the union come up.

\subsubsection{Observations of social interaction as shared experience}

Andriessen's (1978) early work on safety motivation found that a cohesive group works more safely, because "a group atmosphere promotes the development of a positive safety norm so that members are supported in their working safely by the approval of their partner and other colleagues" (Andriessen, 1978: 374). As described in the literature review, most aspects of Andriessen's work was carried on by others into subsequent 
research, however this coworker aspect was not. This research discusses group 'safety norms' in detail in the themes that follow.

As with the physical workplace, social interaction between members reflects a group's underlying cultural structures (Schein, 1985). This social interaction, only partially glimpsed during this project, is the fundamental building blocks of the assumptions and propositions guiding this research. Observing the group interview dynamics and time spent 'hanging around' the locations inform the researcher's perspective on social interaction between workers.

Group interviews were conducted with each of the fire crews and with a group of guides at the rafting company. While originally intended as a content seeking exercise, upon analysis it became evident that the observations regarding social interaction during those sessions were more insightful than the commentary offered by the informants. No group interview could be arranged with the paramedics, however coworker interactions at the base and during shift change provided noteworthy observations.

\subsubsection{River guide social interaction}

The river guides' on-river social environment was very structured and showed a practiced routine. The trip leader set the tone with the guides in a brief meeting before the boats pushed off, and the guides continually looked to the lead boat for direction. Guides relied upon a hand signal system to communicate between boats in the rapids, as the distance and noise were too great for verbal communication. The senior guides appeared to pay closer attention to the junior guides when they were in the rapids, staying closer to them 
with focused attention on their progress. Between senior guides, subtle communication was observed, such as head nods, smiles or eye rolling to make some commentary on trip progress or minor guide slips. When the trip leader signalled to move up or slow down, the whole group reacted. On several occasions, one guide would 'push' another raft into action, by coming up close behind it to get the other guide's attention to get going.

The river guides' group interview was the opposite - very informal. The four volunteers represented a range of experience from first year guide to fifth year trip leader, however given their work and living arrangements they all related to each other both personally and as coworkers. The social dynamic was egalitarian and relaxed as though sitting down with a group of friends. The four often carried the discussion themselves without the researcher's intervention. Within this, respect was given to those with more experience, accompanied by a level of social authority. That social authority was not abused. The senior guides took the juniors' opinion at face value and did not try to speak for them. The conversation was open, as opposed to the directive on-river structure the group used.

\subsubsection{Firefighter social interaction}

Five group interviews were arranged with the urban fire service; one with each crew. These group interviews were conducted prior to individual interviews, yet on the same day. The group interviews varied widely, and became a study in group dynamics rather than collecting diverse opinions on social structure. In two of the group interviews, the captains (recall each crew is led by a captain) dominated the conversation. Those particular captains answered each question first, much of which came across as public 
relations type boilerplate or politically correct answers. For example, the researcher directed a question toward the rookie on the crew. The captain answered for him:

Usually, you [the rookie] are supposed to follow me, that's his job and I am his mentor. He will go around and I tell him what I want. Well everybody, we all work as a team. We have a guy on the ladder, he knows that he has to do something and we start meshing together. We will usually do a face-to-face, if we can, and talk right there. We will get on the radio, if we need extra help. It is all communication and safety is right there with that.

Firefighter (captain)

With this crew, the service members typically echoed their captain's answers:

Everyone on the pump has their own job and they know what they are supposed to do that day; specific jobs that they are supposed to do at calls. We have a chain of command structure that makes everything easy to fall into place.

Firefighter

The individual interviews that followed these group interviews did not follow the same script. Individuals often contradicted their captain in the private interviews, offered with respect to the captain's position and the chain of command, yet providing insight into individual differences:

Yeah and I trust him [the captain], but at the same time you can't help...you know, I have been on the job for a few years and I understand; regardless, he is in charge and he is the boss and he knows more than I do.

Firefighter

The other three group interviews were different in that those captains let their crew speak first and have free reign. These captains did not seem compelled to speak for their crew or offer a company line. One of the captains explained of his leadership style:

You need four sets of eyes to look at every call from a different angle. I am not sure how other officers do it, but I am into [my crew helping me]. If I do not see something, then hopefully [my crew] will and point it out to me. The guys are 
good. I let them teach the [new] guys because they are better at it than I am. They are the ones that will be using the tools. Let them teach it and hopefully the guys can learn from them.

Firefighter (captain)

These same three crews had a more relaxed and collegial atmosphere. Among all five crews, the social hierarchy was visibly tied to years of service. Each crew organized themselves for the individual interviews either by senior to junior rank or vice versa. Yet, the atmosphere in each group was familial. Within the social structure, it appeared individuals were comfortable and respected the relative position of their peers:

To be a team member, you can't get a saw started, then your partner comes over and does it, they are going to make fun of you. The job is still going to get done and it's fine. When I was the most junior at my station, I was always the nozzle person, because that is where you learn the most. Then I got people under me come in, so now I have to step back and let them do the more hands-on stuff. Then, I am there to help them or to do a different task.

Firefighter (six years' experience)

Upon an actual fire call, the crew moved swiftly as a team. In the truck on the way to the call, the backseat firefighters had their head out the window scanning for traffic or signs of smoke to help direct the driver. Once on scene the hoses rolled out with choreographed efficiency. There was much motion and chaos: 7 fire trucks, 3 Chief vehicles, 5 police cars, 2 paramedics and 2 utility service trucks. Yet, the fire crew stayed huddled together and moved as a group. The fire scene had several of these groups moving around under the direction of the Incident Commander (Chief), with no apparent interaction between groups. Individual interviews referred to a sense of competition between stations or crews. The crew's ride back to the station was business as usual. While the ride there was excitement and tension ("This is what we train for" said one 
firefighter), the return did not have a buzz of success or emotional release. The crew returned and got to work cleaning up.

\subsubsection{Paramedic social interaction}

Unlike the other two sectors, paramedics displayed no sense of 'group' (other than identifying as paramedics, as a profession). Shift changes saw two paramedics return and two prep the same vehicle and go out. Other than casual salutations there was little to no interaction between teams. The crew of two disbanded with little ceremony. Often one would leave straight away while the second would file a report at a computer terminal. There was no observed conversation between shifts on highlights or items to carry forward. Friendly banter was conspicuously absent. Workers coming off shift (12 hours) seemed tired, and the going-on shift was focused on task. Between shifts there was no continuity, but even within a crew of two it appeared as two individuals working in parallel rather than together. No social hierarchy was visible, given the limited social interaction observed. Several informants discussed the topic of partner dynamics. Those with a fulltime partner (4 of the 11 informants) recognized clear and positive social relationships, while the casual part-time workers articulated a more difficult social environment:

We were always on the same page, we always worked well together, we had a great dynamic. If she couldn't handle the call no one could because I had that much faith and trust in her that if I missed something she would catch it.

Paramedic (full-time partner)

Some days you get 'that' person. I'm just not a fan [of them], I would never go and have a coffee or a beer together. We have to work together today and that's just the way it is. So you do have those situations that you run into those types of people, so obviously that's going to be a bit of a strained relationship. This is the problem with us in our jobs. I often say it's difficult at times because you probably 
spend more quality time with your partner than you do with a spouse or your family. Then you go through very difficult situations together, you lean on each other and in some ways that can help if you have that relationship, [or] you don't because it's a person I don't really like today.

Paramedic (part-time, random partner pairing)

\subsubsection{Shared experience as foundation for coworker influence}

Shared experience was observed as a foundation piece of all the interview conversations. However, shared experience was only occasionally directly addressed by an informant. More often informants indirectly relied upon shared experience by telling stories from their working past to answer interview questions and to describe social dynamics or coworker influence. Shared experience became obvious as a storytelling culture was pervasive across the three sectors studied.

To be honest, at the kitchen table, sometimes you will hear stories very often that you would think you were there.

Firefighter

There are situations that come up and people talk about things that kind of influence how you see different things. You hear about certain calls and what people did on those calls and then the repercussions from that and you go over what you would do in that situation.

Paramedic

The stories came to be regarded by the researcher as examples of the group's safety norms, pointing towards standards of performance or relative values of safety.

There is a guy here in another shift, actually, he was in a fire and it got really hot all of a sudden and he was 5 feet in the door and he burned all of his ears and they bubbled up. If you don't have [your PPE on properly] you are going to notice. Firefighter

So I was trying to get an IV started, I was trying to figure out my medication dosage and doing all that stuff and at the same time about midway through that process 
it suddenly clicked to me that I completely skipped over the basic airway breathing, and jumped over this sort of thing, and jumped to how I was going to treat this. There are components that had to be done and I looked up and there was [name] already doing all that stuff. He [...] really saved my butt right there. Paramedic

More directly shared experience was referred to as 'time in' together. The time spent in a team setting, especially a team that has been tested and proven capable, was recognized by informants as a key to safety performance.

I think a lot of us encompass a lot of the same values, and the sole reason I say this is because we all have done now two springs of training on super high water. We go through all of April and all of May, we are out there every day no matter what.

Guide

In a team environment, we are very fortunate at this particular station. We have a great crew that really and truly looks after one another's back. We have worked together for quite a while so there is that cohesiveness that brings that job together a lot easier.

Firefighter

Paramedics did not share the same sense of shared experience as did the guides or firefighters. Part-time paramedics, with a new partner every shift, traveled a more individual path rather than a shared one.

As [the paramedics] come down the road they come across a car accident that had just happened. Serious amount of damage, vehicles all over the road, and so one partner said 'Okay, stop and get out we are going to do this', the other one sat in the truck and said 'No we have our mandate from our dispatch so we have to go around this and go to the original call'. So one guy with a few years' experience wants to sit there and not get out of the truck and he didn't. The other guy with 30 some years' experience, gets out of the truck, assesses the patient, it's a drunk driver. Various people injured, it's quite a significant crash.

Paramedic 
So if I personally felt uncomfortable with what they [my partner] were doing I would maybe make a suggestion about doing something else and if they still want to do it well it's not my job to babysit them.

Paramedic

Taking paramedics' shared experience to the partner level, even partner experience is localized and not extended to the larger base setting:

[You learn] something and you go like 'man I just figured out how to deal with this problem'; somebody else goes 'two months ago this is what I did'. So, I have very heated arguments, [...] we have new people coming into this program to work; the knowledge is no good in your head. We to need find a means to disseminate that information.

Paramedic

I would say any progression that happens, happens more inside the truck between two peers. We encountered a situation where we had to self-educate about a policy and now we are both aware of it and even though we will promote it, the other crews won't necessarily adopt that unless they are forced to as well.

Paramedic

The shared experience discussed by informants within each sector were very similar. Firefighters on different crews related very similar social experiences in their workplace. River guides from different companies operating on different rivers related very similar social experiences. Paramedics, too, related similar social experiences however these were markedly more individualistic than the other sectors, and there was a noticeable difference in shared experience between full-time and part-time workers.

The shared experiences either directly or indirectly referred to by the informants enabled sensemaking, articulation of safety norms, and the internalization of safety - all themes discussed in detail below. Shared experience was found to be a precursor and prerequisite to these further themes. Paramedics, it will also be shown below, typically showed less 
support for the themes and propositions. The markedly different social environment that paramedics work in is likely related to the varied support for coworker influence. This idea is expanded upon in the themes that follow.

\subsection{THEME 2: NORMS AS PERFORMANCE STANDARDS}

In organizational literature, norms are described to have a relatively 'soft' texture. In the safety context of this research, norms emerged with much harder lines defining safety performance. Within this theme of norms as performance standards is Proposition 1: Coworker safety norms will represent agreed upon group safety values. These safety values or safety performance standards were based upon the shared experience of the group.

This first proposition was derived from research findings that normative expectations are influential upon individual safety behaviour. Theory involving descriptive and concertive norms were linked together, to propose that coworkers may assemble their collective individual perspectives regarding safety into a group safety culture, one which reflects collective beliefs and values and is manifested in norms. These norms become both context and a constraint upon individual action, and emerged in the data as defined lines regarding expected safety performance.

The present study found qualified support for safety norms being recognized in the workplace, and that those safety norms are established at the group level. 53 of the 55 informants discussed the presence or role of safety norms (see Table 4 at the end of these results). 
There is pressure to perform and be safe. There is an expectation. That is what drives a crew. It is about acceptance and relying on each other like, "You can rely on me, I got your back and you have mine". It is 50-50.

Firefighter

A lot of guides are pretty fresh so they are very impressionable based on what their senior guides do and since most of the senior guides, that we work with here, bought into the [company's] safety system that we have in place, a lot of them see it on the same page as us.

Guide

Interview commentary often recognized both descriptive and concertive norms, although any one individual may have expressed the relative influence of these two differently.

\subsubsection{Descriptive norms}

One view of norms is that they serve as a model of acceptable behaviour (Fugas, Melia, \& Silva, 2011; Fogarty \& Shaw, 2010; Chiaburu \& Harrison, 2008; Jiang, Yuc, Li, \& Li, 2010), an example of a 'soft' interpretation of norms. This role modeling effect was recognized by $89 \%$ of the informants, however what was role modeled was typically expected performance rather than merely acceptable behaviour. In particular, firefighters and guides, who work in intensive group settings, endorsed descriptive norms more often than did paramedics ${ }^{7}$, who work in teams of two.

You know which guys are the ones to watch and not to watch. Most people have a good idea of who works well and who does not. I will take cues from people who have done it [well].

Firefighter

You kind of follow their lead and the lead of senior guys around you to maybe make better decisions that you would make on your own.

Firefighter

\footnotetext{
${ }^{7} 100 \%$ of firefighters, $88 \%$ of guides and $64 \%$ of paramedics endorsed descriptive norms
} 
Those are the people that set the bar, the bench markers, the ones that demonstrate that this is how it needs to be done. Those are the ones with the greatest influence to hopefully motivate others to do well and to do better.

Paramedic

Social learning theory uses descriptive norms - by observing and learning from others, parameters of acceptable behaviour are established (Bandura, 1977). There was evidence of social learning theory in the responses offered, in the form of performance standards:

Sometimes I will come out of a structure; there was a fire and I am inside. I look at the crew across the street and I just know these guys never train. Then, I tell my guys, "See, these guys don't train".

Firefighter

All of the experiences that we've had, bad and good, we talk about them and we make sure that if something bad does happen then we won't do that again. We always make sure everyone's on the same page.

Guide

Some portion of what the individual learns regarding safety is attributed to observing coworkers or interacting with them. These descriptive norms become the bar of performance individuals are expected to meet.

\subsubsection{Concertive norms}

Concertive norms are social rules forcibly applied to in-group members (Barker, 1993, Cialdini \& Goldstein, 2004). This normative aspect of social rules appears to be most influential regarding socially motivated actions, where one's role or status in the group may be compromised (Lapinski \& Rimal, 2005; Manning, 2011). This interpretation of norms has a 'harder' edge than descriptive norms. Concertive norms were recognized by informants as more influential than descriptive norms, as anecdotes of concertive norms 
often became one of the focal points of the individual interviews. Whitewater guides uniformly recognized the power of concertive norms:

I find that sometimes there is a bit of a gap that [new guides] have to bridge and they have to not 'correct it' per se, but we sort of push them in the right direction. Guide

[...regarding a new guide] we are changing him slowly and he is conforming to the way we are.

Guide

Isolating you from the group by not giving you trips down the river, if you were performing poorly, you would get less trips or you would get put on the family trip [...] If you are doing well, positive peer pressure will be placed on you.

Guide

I find sometimes we police [safety performance] between ourselves.

Guide

The whitewater sector operates largely without legislative oversight or industry structure, with the result being safety standards were socially enforced at the coworker level.

Firefighters and paramedics, both highly regulated sectors, still recognized concertive norms and social pressure to perform safely ${ }^{8}$ :

I've seen guys kind of get chased out of the station. On the negative side if they were not a good fit or not performing to the expected level, a lot of very negative attitudes float around.

Firefighter

...they are the people who will pressure them into conforming to the way it should be, like up your game a bit.

Paramedic

\footnotetext{
${ }^{8} 89 \%$ of firefighters and $91 \%$ of paramedics recognized concertive norms and social pressure to perform safely
} 
Within the discussion of concertive norms, some paramedics discussed how they would not enforce safety standards on others. A common refrain was 'I'm not their boss', referring to a partner's safety behaviour. This implies that a safety standard does indeed exist, but in certain circumstances workers will not socially enforce these standards upon others:

...as long as it won't hurt me or my patient, I'm not going to tell the person who has been here for 30 years we're not moving this ambulance until you put your seatbelt on.

Paramedic

For paramedics, social credibility emerged as playing a role in concertive norms. As paramedics work in teams of two, the credibility of the partner was discussed as being relevant as to whether they could enforce normative standards upon their partner.

It's really about the influence that that peer has. What is their popularity within the group? If it is someone that no one likes then they're not really prone to listen. It's an unreliable safety source for sure.

Paramedic

At one of the workplace visits at a paramedic base, one (part-time) paramedic unsuccessfully tried to convince his partner for the day (another part-time) to participate in the research interviews. The uninterested partner could be described as 'cold' to the idea, and turned his back on his partner and returned to his computer screen (looking at internet news). It seemed clear that the interaction had more to do with the credibility of the first partner and the non-relationship between the two rather than the actual participation in the interviews. 
The relevance of concertive norms depended upon the tenure of the worker. $54 \%$ of the coded comments regarding concertive norms came from workers with six to 10 years of experience $^{9}$. It seems that at this middle tenure juncture, individuals recognized that they now enforce rather than receive social pressure:

I think [peer pressure] depends on the point you are in your career. I see it differently than I did six-years ago when I got hired.

Firefighter (six years' experience)

If they are a younger guide who could be dealing with the exact same things as the older guide, they are usually made an example of. I think that younger guides are held to more of a standard than the older guides.

Guide (eight years' experience)

The nature of the interview discussions regarding concertive norms placed them as more active and more powerful than descriptive norms. This aligns with Barker's (1993) original conception of the construct, that norms are forcibly applied by in-group members. There was evidence of concertive norms as both context and constraint, where it enabled positive performance and put pressure on those who underperform. Concertive norms were used as both social expectation and a performance standard. The overall tone regarding concertive norms is that they are a positive and necessary force, especially in the guiding and firefighting sectors. Guides use concertive norms to establish and uphold safety standards, while firefighters use concertive norms to shape the social team that spends 24 hour shifts together and collectively looks out for each other. Paramedics discussed concertive norms, but their influence was regarded as less. Paramedics were more likely to discuss how they would not enforce norms rather than push them on their

\footnotetext{
${ }^{9}$ Workers with 6-10 years of experience represent $30 \%$ of the sample group yet contributed $54 \%$ of the coded commentary on this point.
} 
partner. For paramedics, safety norms still existed within the prescribed standard of care obligations, but as a social tool, an appropriate relationship was not in place to enforce them.

\subsubsection{Norms as agreed upon values}

The core premise of Proposition 1 was that workers would assemble their collective safety perspectives into an agreed upon set of safety values. This proposition was supported in the data, albeit with conditions ${ }^{10}$. All the firefighters and guides reflected the ideal of agreed upon safety values. Most paramedics supported the proposition of shared safety values, while some made it clear there was a lack of agreed upon safety values. Agreed upon safety values were evident in the discussion above regarding descriptive and concertive norms, but were also discussed in terms of rules and safety performance standards.

Some informants offered agreed upon safety values as rules:

In our crew, everyone knows you cannot rush into it [a fire call]. Firefighter

You stick by your partner.

Paramedic

Some of these safety rules appeared to have become mantra: every single firefighter $(n=$ 28) stated his or her single most important safety practice every day was checking their PPE. There was no variation or hesitation in any informant - an example of group values becoming safety mantra. While there was variation in opinion as to how thorough

\footnotetext{
$1093 \%$ of responses either directly or indirectly supported Proposition 1.
} 
coworkers were in performing this task, there was no doubt that the task was prioritized by all.

More generally, there was clear support for Proposition 1 in regards to expectations regarding safety performance standards. For example:

Guys never slack because we do things a lot in group activities. If one guy is less on the spectrum, I think he will step it up, if everyone else is. Generally, that is the case. As opposed to guys going backwards, wheeling it down. I think the guys on my shift and crew, tend to step it up more, if the other people are.

Firefighter

It has been quite interesting watching a whole bunch of new people come in and assimilate into a particular kind of culture and maintain that and then I think it stands out quite obviously, when one or two people that have an indiscrepancy [sic] over the cowboy tendency and they stick out... and it becomes quite obvious that is not the norm and it is not the expectation.

Guide

Through the informants' words it appears that any new worker joining the firefighting or raft guide sector would sense that there was a peer standard and normative expectation regarding safety values. As a specific example of socially negotiated safety values, the fire sector was adapting to recent requirements to wear a seatbelt on the way to a call. This was cited by a number informants as something that was a point of contention for several years, as the seatbelt interferes with putting on the air backpack. While there was still variance on follow through, the practice itself has appeared to have gained group value.

It has been improving since I have been on the job. More people are wearing their seatbelts. You can get on the truck, put your seatbelt on and go. It takes 10seconds to throw your shoulder straps on then down and put your waist belt on. Firefighter 
Okay, I do not wear a seatbelt on the truck. I am the only one that probably does not. I do wear it every day in the car. Consciously, we have [historically] not worn them and I do not wear them. I do think of it often. Sitting there and think "I am the only one, not wearing their seatbelt?"

Firefighter

On the one ride along to a fire call that the researcher observed, only the driver wore a seatbelt. The captain (front seat) and two firefighters in the back seat did not put on a seatbelt. It did look like it would be cumbersome to wear a seatbelt, as the two firefighters in the back seat (where the researcher was also sitting) were moving about, in and out of their seat, collecting gear and doing up equipment, eventually standing at the door window to scout smoke to help the driver find the address ${ }^{11}$. In individual follow up interviews with these crew members, in 3 of the 5 (one of which was the captain) seatbelts were discussed. Seatbelt use was rationalized as risk versus reward, or alternatively, production versus safety. The benefit of wearing a seatbelt did not outweigh the difficulty and risk it created in efficiently putting on PPE.

I will risk the rule of the seatbelt because I'm more concerned about my safety when I get in that fire. I'Il risk that 3 minute drive because when I'm going in I will get burnt if [I'm not done up properly].

Firefighter (captain)

[Seatbelts are] managed the same way we manage everything else in that each one of us makes a calculated risk-benefit analysis on every aspect of the job. If you decide to be complacent or cut a corner that deviates from policy, that it's done knowingly and that the intent is to achieve some sort of net benefit. Now

\footnotetext{
${ }^{11}$ As an example of social influence, as the researcher I did not put on a seatbelt on the way to the fire call. My first intention was to do so, but getting into the seat (the back portion of which is actually an air backpack) it was not immediately obvious how to put it on. I then looked to the firefighters in the backseat with me for cues, but they didn't have theirs on, so I did not pursue it any further. It felt somewhat exposed being in a fire truck without it (given that I always wear a seatbelt in a car) but was rationalized as 'normal' in an environment that I had zero experience with. This rationalization was experienced on the first ride, underscoring the power of social influence on perceived safety norms in a group setting (even for an outsider).
} 
do I think it's right? Sometimes. Most of the time it's just complacency that just turns into the routine.

Firefighter

Regarding safety as agreed upon values, this crew, either under the leadership of the captain or by consensus, arrived at a position on seatbelt use relative to safety. Another crew, at the busy downtown station, voiced clear consensus in the other direction driving safety was important, and seatbelts were a part of that.

When you go out 15 times a day, just driving through traffic here. There is a [potential] disaster for every call, so my perspective is to slow down and take your time.

Firefighter

Paramedics were found to not have agreed upon safety standards. This is noteworthy given that they are the most regulated sector in this study - safety performance is explicitly stated in Ministry of Health guidelines and standard operating procedures (SOP).

Yet paramedics had the least consensus on and social enforcement of safety:

I think everybody relates differently to what's safe and unsafe. In the morning when you do your truck check, some people just glance at it and say 'Oh ya, we're good'. Whereas it would be unsafe for the patient if you didn't check if you had oxygen. Everybody is different.

Paramedic

Part of the problem is that some people don't have a true understanding of safety. Whether it's they've just not been exposed to situations or they're not cognizant of the threats or whatever. We have a coworker, who still works here, who wrestled a man swinging a sword... Brilliant.

Paramedic

Relative to Proposition 1, paramedics appeared to have widely divergent safety perspectives. Seven of the 11 paramedics perceived there to be shared group values regarding safety while the remaining four did not. This divergence leads to the finding 
that paramedic safety perspectives have failed to coalesce into agreed group values. The work environment of isolated pairs was recognized by paramedics as an impediment to positive social influence. Two paramedics, often paired only for the day, spend their time in their own ambulance and have no interaction with other paramedics. There were fewer opportunities to socially negotiate or agree upon values, and there was no real 'group' to speak of:

One day we came back to the base and it was one of those situations where the night crew basically rolled in late and we went out to switch over the vehicle and the vehicle was completely upended and filthy and so I jumped in the back putting all the medical kit back together, restocking medications. [My partner for the day] gets the hose out and starts washing the vehicle because that is what is important to [him] is that we look good. I lit into him in the middle of this 'Hey get the stuff inside sorted out first then we can worry about how pretty the truck looks'. Him and I were having an argument and in the middle of that argument tones go off for the next call, which made my point.

Paramedic

So I would say any progression that happens, happens more inside the truck between two peers. We encountered a situation where we had to self-educate about a policy and now we are both aware of it and even though we will promote it, the other crews won't necessarily adopt that unless they are forced to [learn] as well.

Paramedic

One particular performance standard is shared between emergency sectors: out-the-door times when a call comes in. Paramedics are provincially mandated to be out the door and on the radio in 90 seconds. This was expressed as difficult by several informants.

As soon as we get paged out that we are going to a car accident, the first thing we should look at doing is do I have the appropriate PPE before I even get into the truck. But again, we are pushed that we have to be on the radio booking and pulling out of the garage in 90 seconds. 90 seconds does not give you time to do that sort of thing. 
Paramedic

Observations of call responses during paramedic site visits showed a surprisingly languid response to a call chime. One paramedic was observed, upon a call coming in, to walk down the hallway to the kitchen to retrieve a lunch from the fridge and fill up a water bottle before going to the garage to climb in the ambulance. Compare this to firefighters' habitual PPE checking in the morning and getting a crew of seven people dressed and out the door in 20 seconds. One firefighter offered:

My personal philosophy is we are going to treat every call like we are going to one of our family, my kid, my grandmother, mother or sister, so when the bell rings I expect [us] to hustle. There is nobody toddling here and that's the way I treat every call.

Firefighter

As a means of comparison, both a paramedic and a firefighter informant explained that $90 \%$ of their calls were very routine and $10 \%$ were exceptional or critical. Observations of the paramedics' response to a call chime is that they were responding to the expected $90 \%$. The firefighters were responding to the potential $10 \%$ every time the alarm sounded. These two perspectives were very different takes on the same task, and are an example of how safety norms become performance standards, agreed upon and adopted based upon the shared experience of the group. As a paramedic, there is no use hurrying into the ambulance if your partner is going to use their full 90 seconds they are allowed before they join you. Combine this with a culture that is reluctant to apply coercive pressure in a temporary partner relationship, and this norm becomes self-reinforcing.

In summary, safety norms were clearly present in all three sectors. Both descriptive and concertive norms were recognized by informants as playing a role in shaping ones' own 
safety performance and safety beliefs. Firefighters and guides showed clear support for Proposition 1 and could articulate how coworker values became assembled into group values regarding safety. These values got modeled via descriptive norms and enforced via concertive norms. Paramedics did not show consistent support for Proposition 1, raising the possibility of contextual factors or moderating variables.

\subsection{THEME 3: SENSEMAKING AND AMBIGUITY REDUCTION}

In safety science, sensemaking has very little presence at either the group or the individual level of analysis. It was not targeted as part of this safety study, however it clearly emerged as an active mechanism for coworkers to help individuals understand ambiguity in their job. Sensemaking is well represented in literature at the organizational level, with academics such as Weick $(1993,1999,2010)$ using failures in sensemaking to explain high profile organizational accidents. Sensemaking was defined earlier in this paper as an ongoing, socially based interpretative process that constructs interpretations

of ambiguous situations (Brown, 2000). Sensemaking is one means of coming to terms with role ambiguity and role conflict, where Chiaburu and Harrison (2008) found that coworkers play a key role.

Paramedics, in particular, face significant role ambiguity and role conflict. Firefighters are confronted with role conflict, and river guides provided evidence of retrospective sensemaking. 


\subsubsection{Sensemaking and ambiguity in paramedicine}

All the paramedics $(n=11)$ discussed the presence of ambiguity and role conflict in their job - it would be the defining feature of this sector in this study. Next to discussing social identity as a paramedic (a targeted theme in this study), sensemaking and ambiguity was the second most coded theme for this group. The paramedics in this study referred to two issues: ambiguity in authority and conflicting priorities.

\subsubsection{Who is in charge?}

Recall that paramedics work in teams of two, both with equal authority with no assigned leader. Eight of the 11 paramedics recognized that it does not really work this way:

It's almost that elephant in the room but there is always a leader and a follower. It might not want to be defined as that and they may not want to admit it outwardly but if they are honest [...] he has more experience, has a higher level of care, more dominant personality [then he is the leader].

Paramedic

Historically and currently on paper the paramedics 'swap' who attends the next call, but there are many variables that change this on a call-by-call basis, especially with the advent of advanced care paramedics:

You have the conversation who's attending or who's driving first because it switches back and forth. Except for ACPs sometimes depending on the priority of the call they will have to be in the back, which is governed by the base hospital.

Paramedic

I find myself very much doing the assist role [as a PCP]. What can be very disconcerting for some people is that as a PCP they do the assist role so much and then they get thrown with a PCP partner and now you're top dog.

Paramedic 
Being a highly-regulated sector, there are several oversight bodies in paramedic services. The provincial Ministry of Health provides very specific guidelines regarding standard of medical care, response times, and time on scene, according to the informants. These guidelines are ultimately the standard against which a worker is assessed. Paramedics have a direct employer, in this case a county administration, to which the worker is also accountable. Additionally, a paramedic service works under the authority of a 'base hospital', with a duty physician who expects to be consulted on any serious drug treatment required. It is the base hospital that receives all call reports and is expected to flag questionable application of Ministry of Health protocols. There is an independent provincial dispatch centre that receives 911 calls and directs the crews' workload. Of all of these bodies, a crew interacts most with the dispatch centre and base hospital, neither of which are their employer, yet will be the bodies to report the worker for substandard performance.

So on scene you've got maybe four bosses and three different regulations or legislation that are informing your decisions. You have to be able to parse that out. Paramedic

I would say it is difficult at times to filter out what one [body] wants versus the other.

Paramedic

The medical 'lead' on each call is unknown until the crew can assess the severity of the situation, which combines with the ambiguity of working with a different partner every shift (for part-time workers). Plus, the various layers of authority have expectations regarding performance, plus very complex medical problem solving and environmental factors that complicate task execution and safety. 


\subsubsection{Conflicting priorities}

With all of the ambiguous layers of authority, informants easily provided examples where those layers produce conflicting priorities - conflict that is up to the paramedic to align while trying to respond to stressful situations.

Is base hospital going to come back at me? I just did a chest pain call and I show up at the house and there is a little porch with two steps up to go into the house and [the patient] comes walking out on the porch and as I was coming up the steps he is coming out the door and my stretcher is 2 steps down and I made him walk. Well right, our protocol is you never walk anybody. It's two steps. I grabbed his arm and walked him down the steps. I documented that he walked so potentially I could hear back on that. But what else would you do with him, pick him up and carry him down two steps when the momentum is already there?

Paramedic

Everyone that watches us feels like it's very black and white but it's not. Every call, even for the same complaint, ends up being different because the house or the patient is different or the family members on scene are different or their living situation is different so there are always little hoops that you have to jump through that put you in the grey.

Paramedic

The result of conflicting priorities and ambiguous authority is individual sensemaking and tactics to manage the conflict. One common tactic discussed was doing the minimum requirement only, so as not to take any chances and potentially get oneself into trouble:

And it's amazing that we don't screw up more often [with all the conflicting priorities] ... a lot of what my partners do is they turtle. There is a policy that says this; [then] this is what I'll do.

Paramedic

Others discussed ways of navigating the system to get the best outcome for their patient.

For example, one paramedic explained that by manipulating the severity of the medical condition reported back to dispatch, the paramedic could deliver the patient to the hospital they knew better suited the patient's needs. Just as they approached the desired 
hospital, the paramedic would call dispatch and raise or lower the severity to its actual level. When asked if all paramedics know this practice, the informant responded:

A: No that comes from experience.

Q: So why isn't there a mechanism to pass around that here is the way you really do it because it works better?

A: Because it's completely against all policy set by the Ministry.

Q: So you can't really talk about it?

A: It's completely illegal. You can't do that.

Paramedic (20 years' experience)

Paramedics discussed the sensemaking required to manage ambiguous authority and role conflict whether full-time or part-time. Full-time paramedics had the advantage of a consistent partner with which to share the sensemaking. Part-time paramedics had to deal with the added social pressure of differing partners every day.

\subsubsection{Sensemaking and ambiguity in firefighting}

As explained in Theme 1, firefighters work in a very different social context than paramedics. Authority and chain of command was very clearly laid out. There was no ambiguity reported in this sense. On the other hand, firefighters respond to highly dynamic, life threatening situations. Coming to terms with this risk, firefighters came to shared agreement regarding safety priorities (Theme 2). There is an element of sensemaking to arrive at this balance between risk and safety ${ }^{12}$.

\footnotetext{
${ }^{12} 64 \%$ of firefighters discussed either sensemaking in an uncontrolled environment or in managing conflicting priorities.
} 


\subsubsection{Elements of control in an uncontrolled environment}

The firefighters' safety mantra of checking PPE can be interpreted as a means of controlling one variable in what is often an uncontrolled situation (a fire). In this way safety becomes a form of ambiguity reduction. Several firefighters addressed acceptance of the open-ended nature of safety in their job. Due to the uncontrolled nature of the environment, even proper safety performance does not guarantee safety.

So, yes, your safety is number one and we do anything, as much as possible, to keep that a priority. In this job, in my opinion, it is impossible to say that your safety is always going to be number one, because it's not, it is impossible with the nature of what we do, it is impossible to know every factor to control that.

Firefighter

It is a dangerous job. Something unexpected could collapse, but bottom line, if you play it safe; do a 360, go in as a group, bring a hose line in and the guys outside put ladders at windows, we should be safe. We are making a bad environment safer. That is all we can do.

Firefighter

\subsubsection{Conflicting priorities}

Informants discussed different levels of sensemaking with regards to conflicting priorities.

All the firefighters contributing to this theme discussed how they interpreted inevitable conflict between policy and getting the job done:

So what they [management] do is they say 'do this' but they know we are not going to follow it because we are just not. The whole thing is we are going to look after that kid like it's our own kid. So there are all these rules designed to protect us, that they know are going to be broken, so now the entire department [knows] the rules don't mean anything because the rules are so far-fetched.

Firefighter

Realistically, this is how is plays out, you are either a hero or in trouble. It is one or the other. Things worked out well. We got a pat on the back and there was a 
lot of brass happy how things turned out. If things don't play out that way, I don't think we are getting a pat on the back and I am thinking we are probably getting some days off. That said, we broke many rules, but any rule that we had any control on, in the situation, we followed it to a tee. The problem was that the rules we did break were the big rules.

Firefighter

A smaller group of firefighters ${ }^{13}$ discussed more existential conflict. It was explained that the public expects firefighters to put themselves in harm's way, yet within the fire service the priority is individual safety. These two priorities are fundamentally at odds. The balance between safety and doing the job the public expects was often summed up as 'risk a lot to save a lot'. Beyond that, there were hints at the unease that exists in this grey zone:

I think [balancing risk] comes from the individuals that have actually suffered huge disappointments and not doing something that they should have or could have. The problem is that it is never identified, beforehand. None of the young people are told, "Do you realize how you are going to feel if somebody is screaming and you don't go and get them?"

Firefighter

\subsubsection{Relying on coworkers for sensemaking}

Among these discussions, the informants reliably looked to their coworkers for direction.

Being able to rely upon coworkers whom they trusted reduced some of the ambiguity of uncontrolled situations. Discussing ambiguity with coworkers also aided in individual sensemaking.

When I hear a new sound, I think 'this is bad' because we are going into a bad situation. I hope that we are all going to say the same thing or someone will say, "I have heard that before". Then, we can continue that much further. Firefighter

\footnotetext{
$1339 \%$ of respondents.
} 
I could feel something telling me, you need to get out of there. I was looking at the officer, and I was there with another guy, I turned to him and said, "Look at this!" and he said, "Yeah, we should get out". We got out and not even 30 -seconds later, the building collapsed to the ground.

Firefighter

Firefighters employed sensemaking to deal with the ambiguities of an uncontrolled environment, the conflict between the rules and getting the job done, and at a higher level the tension between taking care of one's safety and the pressure to take big risks.

\subsubsection{Sensemaking and ambiguity in whitewater guiding}

Ambiguity was less present in the guiding sector compared to the other two sectors. Informants discussed retrospective sensemaking with the role of staff debriefs in establishing safety norms and performance standards. The nature of the work itself presented very little ambiguity. The work of dealing with clients and steering the boat through rapids was considered straight forward even though it is not bound by regulations.

Of the guide informants discussing sensemaking, most were the more experienced guides - the trip leaders and decision makers. Commentary was provided that established rookie guides as just following rules, while senior guides interpreted more leeway. The group interview discussed this, with the junior guides learning for the first time that what they thought were rules are actually judgement decisions by trip leaders:

For us it seems like when the water level drops it is a clear-cut decision, but for you [trip leader], you see more of those border line time frames.

Guide 
In summary, ambiguity is a defining characteristic of the paramedics' work world, and continual sensemaking is required to manage safety performance. In particular, the ambiguous authority creates conflicting priorities and a sensemaking response of either doing the minimum or else subverting the rules. Firefighters have a highly structured social environment which aids in their sensemaking regarding the uncontrolled situations to which they must respond. In this sector, ambiguity arises between safety policy and the realities of getting the job done, plus the more existential tension between one's safety and taking risks for others. That firefighters work with trusted crews makes this sensemaking easier in high exposure situations. River guides did not identify ambiguity as a large factor affecting their safety performance.

\subsection{THEME 4: SOCIAL IDENTITY WITH THE GROUP}

Social identity theory proposes that individuals categorize themselves according to the social groups in which they consider themselves a member (Tajfel, 1974; Haslam, 2004). Social identity theory also describes how situational factors can influence identification between individual or group membership. Work settings with high task interdependence (Stark, Shaw, \& Duffy, 2007), a positive history of group success, or external competition likely lead to strong group social identity (Gibson \& Early, 2007). In this context, a group with strong social identity would be one where all the members of a work group selfidentify as 'we', and a weak group social identity would be one where some or all members default to ' $I$ ', even when bound in a group setting. 
Theme 4, social identity, was a targeted theme in this study. This theme includes Proposition 2, that individuals working in safety critical or highly safety focussed groups will have high safety oriented social identity with the group. This proposition assessed the individuals' social identity with regards to safety and with regards their work group. Proposition 1 was assessing group norms, while Proposition 2 is assessing how the individual identified relative to their group.

The findings support Proposition 2. Almost all the firefighters and guides expressed a social identity that aligns with the safety priorities and identities of their group. However only half of the paramedics self-identified with their group's safety values ${ }^{14}$ (see Table 5 following these results). Individuals with strong safety-oriented social identity recognized their own role in safety performance and how that contributed to group performance or safety outcomes. Beyond performance, these same individuals intoned or provided evidence that they had adopted work identities that were related to their ability to deliver on safety priorities.

I put my safety along with my crew's safety together as one. I am not going to look out for myself more than I would for somebody else. I take things in a team perspective. Crew safety comes first.

Firefighter

I think because we are such a tight crew, we all kind of have a similar mentality going about [safety], but I also do believe that we are all pretty good. Guide

\footnotetext{
${ }^{14} 93 \%$ of firefighters, $100 \%$ of river guides, and $55 \%$ of paramedics identified with their group's safety values.
} 
Factors identified in social identity theory as contributing to strong social identity emerged in the interviews. There was variation in individuals speaking from 'we' or from ' I', as well as reference to a group history of success. Task interdependence was present in all the sectors studied.

\subsubsection{We versus I}

In some settings individuals pursue their own goals and identify first and foremost as ' $I$ ', or in other settings can view themselves as a member of a group, and identify as a part of a collective 'we' (Ellemers, de Gilder, \& Haslam, 2004). Proposition 2 proposed that in a safety oriented work setting, individuals should self-identify with the group and tend towards using 'we'. Among firefighters and guides this was the case. All the guides almost exclusively used 'we' to describe their individual work, their safety priorities and their own duties and obligations regarding safety. Among firefighters, the majority primarily used 'we'.

At the end of the day we all really understand how tough this [job] can be sometimes.

Guide

There is still so much that we can't control in what we do.

Guide

If we can keep things safe and fun at the same time it makes our life easier and it makes the clients life a lot better because they get the enjoyment without the risk. Guide

Those are all big things [rules] we broke, but any rule that we had control on we justified with. As much as the SOP's were right, we should have waited but there was no time.

Firefighter

Only four of the paramedics used 'we'. These were the four with full-time partners. 
We were always on the same page, we always worked well together, we had a great dynamic.

Paramedic

The part-time paramedics, who are tasked with a new partner every shift, all used ' $\mathrm{I}$ ' exclusively:

I know that if I make a mistake but I make it in a valiant effort and I've done everything I possibly could have but the results were still negative that I'm going to be hung out to dry.

Paramedic

Cover your own ass; make sure your paperwork is done. I don't overwrite my paperwork and I try and keep it simple and concise.

Paramedic

Through social identity theory's 'we' versus 'I', language becomes a lens to view individuals' self-categorization with a group or whether they perceive they are working on their own. By using 'we', the majority of informants express support for Proposition 2, in that they self-categorized with their group's safety priorities. The paramedics who used ' $\mathrm{l}$ ' would all claim identity to being a paramedic, but their words indicated their view as being on their own rather than a part of a team or group effort. This aligns with previous findings regarding paramedics' shared experience in Theme 1.

\subsubsection{Task interdependence}

The nature of the safety critical tasks of the sectors under study lent themselves to teamwork. Task interdependence is considered a factor contributing to strong social 
identity with a group. Informants from all three sectors recognized the interdependence of their and their coworkers' roles in contributing to safety ${ }^{15}$ :

We are very concentrated on the job we each have to do, but at the same time, we are not looking straight ahead, we have to look to our sides in order to see something that someone else may not have. We are looking out for each other. Firefighter

Most of the time you are right there working with them so if they are not doing something you just do it, it is a partnership. If they are not putting on a blood pressure cuff you go ahead and put it on while they are talking to the patient.

Paramedic

It was a co guide who was angry with me and he said 'you've got to look out for me and everyone behind you' and I was like, 'oh right, sorry' he repeatedly said that until I finally got it.

Guide

These comments inferred a level of commitment to safety performance, and a level of self-identification with group goals regarding safety. The task interdependence evident in the data supports Proposition 2 and individuals' self-identifying with their group.

\subsubsection{History of group success}

A final factor that theory predicts will contribute to strong social identity is a history of group success (Haslam, Powell, \& Turner, 2000). This factor was less evident in the data compared to previous factors considered, however for whitewater guides it was common to discuss past experience and how that contributed to social identity. To a lesser extent firefighters referred to past team success which could be related to social identity ${ }^{16}$.

\footnotetext{
${ }^{15} 89 \%$ of firefighters, $100 \%$ of guides and $91 \%$ of paramedics recognized task interdependence for safety.

${ }^{16} 75 \%$ of firefighters, $100 \%$ of guides and $45 \%$ of paramedics referred to past team success.
} 
We have a very good track record for safety and I think it comes down to the fact that it is sitting in the front of all the employees' minds.

Guide

We have one of the busiest stations in the city, so it is where a lot of people want to go. If there is a list of top stations, we are usually one of the top three. People know, if you want to be here, you have to show it and you have to pull your own weight, otherwise, somebody else that is going to try will take your place.

Firefighter

Fewer paramedics discussed past success as informing their safety identity. Most of the paramedics interviewed did not have a dedicated team, so had little in the way of a shared history upon which to draw. Their history is their own, rather than shared. Social identity theory identifies an additional factor, competition among groups, as contributing to strong social identity. There were no significant findings regarding 'other' groups that emerged in the data.

\subsubsection{Safety oriented social identity}

Guides and firefighters expressed strongly endorsed social identity to their group regarding safety. Just over half of paramedics expressed conclusive social identity with their group ${ }^{17}$. The remaining paramedics that expressed low or no evidence of social identity also tended to speak from the position of ' $I$ ', as discussed above, while those with strong group identity defaulted to 'we'.

Some examples of comments that inferred self-identifying with their group's safety values:

\footnotetext{
$1793 \%$ of firefighters, $100 \%$ of guides and $55 \%$ of paramedics strongly endorsed social identity to their group regarding safety; the proportion of relative strength of endorsement is further broken down in Table 5b.
} 
We are training every day making sure we are good. We are all about being consistent in our values and the safety stuff we do. We are such a small tight group of guides that we all understand.

Guide

A lot of our feedback about why people choose us is because of our safety standards. So, I think it's something we try to emulate all the time.

Guide

A lot of us have a "type A" personality, the teamwork personality; we like to get things done. If a job needs to be done, we either do it as a team or step up and do it. As a firefighter, I would say that team atmosphere, but all parts of that team need to work for the whole team to succeed.

Firefighter

Firefighters are sharing what we know and getting smarter amongst ourselves. It is a powerful way of learning. Learn from each other, as opposed to waiting for it to come down from the safety department.

Firefighter

Firefighters are known, anecdotally, for their tight social units. Many firefighters discussed the family aspect as key to a work crew's success. Being in a dedicated team that shares 24-hour shifts allows for strong social identity:

You get to be really close with those members, they become your family and that's really important, like they are brothers. When you are on a call, we are really looking out for each other's safety and that's always number one, on every call we go to.

Firefighter

This is in opposition to paramedics, only a small portion of which had a dedicated partner. One of the paramedics with a full-time partner recognized the difference in social identity between work sectors:

The difference between us and firefighters is you put on a firefighter T-shirt and you go anywhere in the world and another firefighter will say 'Hey brother' and they chit chat. It's not like that with paramedics.

Paramedic 
As found in Themes 1, 2 and 3, the social aspects of paramedic work prove to be different from firefighting and whitewater guiding to the extent that strong safety oriented social identity is less common. This proves consistent with findings from Proposition 1, in which paramedics did not show consistent support in agreeing upon socially created safety norms.

In summary, Proposition 2 is supported by the firefighting and guiding sectors, but could not be considered supported by paramedics (as only half positively aligned). The factors contributing to strong social identity (as identified by social identity theory) were present in both guiding and firefighting: referring to ' $w e$ ' in reference to individual safety orientation, high task interdependence, and relying upon a history of group success. The family aspect of firefighting work crews became an appropriate analogy for selfidentifying with a group. Almost half of the paramedics did not identify with group safety priorities, even though their sector is highly regulated from a safety perspective. Even with task interdependence, part-time paramedics still primarily spoke from the perspective of the individual rather than a team, and did not reference past success as informing their safety perspective.

\subsection{THEME 5: INTERNALIZING SAFETY}

Theme 5 was targeted in advance to directly address this project's research focus of coworker influence and the internalization of safety. This theme includes Proposition 3: Given a strong social identity, the individual is more likely to adopt and internalize coworker safety values rather than externally regulate safety values. 
So far, this study's findings point to shared experience and individual sensemaking enabling shared understanding regarding group safety priorities and norms. Does this understanding allow for or ease individual internalization of safety priorities?

By indicating social identity as a qualifier to adopting and internalizing group values, Proposition 3 includes the key concepts that comprise SDT. SDT moves group outcomes from an imposed goal or external form of motivation (i.e. norms) towards voluntarily adopted intrinsic motivation. SDT represents a process by which coworkers influence the individual's social identity, which then becomes incorporated into self-concept.

Internalized safety motivation means that performing to a safety standard is wholly volitional and represents personal significance attached to safety. Safety performance aligns with the individual's self-concept and values, even though the activity is not inherently interesting (i.e. not inherently intrinsically motivating). Externally motivated safety is controlled - pressure is applied to act in a certain manner - that relies upon reward or punishment for compliance (Gagne \& Deci, 2005).

Almost all the informants described their safety orientation as internalized ${ }^{18}$ : their own safety performance was meaningful to them and safety became a self-selected goal (see Table 6 following these results). This internalized safety motivation lends support to Proposition 3. External threats or rewards regarding safety were minimally influential for some individuals or non-existent for many as motivation to perform safely. External

\footnotetext{
${ }^{18} 96 \%$ of informants described internalized safety motivation. Only the 48 individuals who expressed strong social identity in Proposition 2 are considered for analysis and assessing support for this proposition. This is detailed in Table 6.
} 
motivation with a social aspect - conforming to normative expectations - was present in addition to internalized safety in much smaller portion of the informants ${ }^{19}$; only one respondent indicated clear external pressure as the primary source of their safety motivation.

Internalizing motivation can be promoted, according to SDT, when an individual has a sense of competence for the act, understands why and how the act relates to the larger context ('relatedness'), and has some choice and autonomy in execution. As a last social factor, internalization is facilitated by "explicit or implicit endorsement of the behaviour by significant others" (Gagne \& Deci, 2005: 339). Coworker support for safety, SDT posits, enables the internalizing of group values. These variables are examined in turn.

I have seen people change from "Who cares" to being all in [when it comes to safety].

Firefighter

\subsubsection{Belief in safety competence}

A sense of competence allows for ownership and responsibility for one's actions (Gagne \& Deci, 2005: 332). Only two of the informants expressed reservations in describing their own abilities in regards to safety performance ${ }^{20}$. All the firefighters and paramedics included in the analysis of this proposition were confident in their ability to deliver on safety:

Some guys, [when driving the fire truck to a call] ... at intersections they stop and go. There are guys that are more strict, in that way. I am not one of them,

\footnotetext{
${ }^{19} 17 \%$ of the informants recognized social pressure as external motivation in addition to internally motivated safety (see Table 6b).

${ }^{20}$ Both individuals who expressed reservations of their safety competence worked as whitewater guides, both were female, and had two and six years' experience, respectively.
} 
obviously, I stop, but if it is not a huge reason for me to do a complete stop, I will just tap the brakes and keep going.

Firefighter

I'm sure other people think that the top guides are the ones that never have bad things happen to them, or they never flip or they never do anything [...] I think a top guide is defined when things go wrong not when things go well and how well they perform then.

Guide

[Part-timers] like working with me because they actually reduce their cognitive load knowing that I'm a highly competent paramedic.

Paramedic

\subsubsection{Relates safety to conceptual outcomes}

Self-determination theory identifies relatedness as a variable encouraging internalizing a given value. From a safety perspective, understanding how one's specific safety actions contribute to larger goals or outcomes would be an indication of this relatedness. Firefighters, guides, and paramedics expressed understanding of this relatedness ${ }^{21}$ :

I am not going risk my own safety for a dumpster fire, it's not like there is a child in there or someone I have to rescue, it is just a burning dumpster, I am going to look after myself.

Firefighter

I would like to think the ultimate reason for [guides] being safe is just their moral compass; no one likes to see anyone get hurt.

Guide

...basically the guidelines are there but I'm going to apply them, it's not black and white policy, its principles, its theories and concepts that you apply to any dynamic situation for guidance but you know you are still going to act in the best interest of the patient and the best interest of safety.

Paramedic

${ }^{21} 77 \%$ of firefighters, $100 \%$ of guides, and $83 \%$ of paramedics expressed understanding the relatedness of their actions with regards to safety. 


\subsubsection{Autonomy to pursue safety}

The defining characteristic of internalized motivation is that it is autonomously controlled (Gagne \& Deci, 2005: 333). Autonomy implies volition and having a choice regarding safety performance, as opposed to externally regulated behaviour. All informants expressed autonomy with regards to pursuing safety:

Sometimes I will look around and nobody has their [air] mask on and I think, "I want to put my mask on", so I will do it.

Firefighter

The problem is when you are taught those steadfast rules, you can't think. Firefighter

I do what is in the best interest of the patient. I'll do what I have to do in that situation and I'Il deal with it [consequences] later.

Paramedic

Five of the rookie firefighters described their autonomy as low. In their training role they follow the captain's lead at all times and are not allowed to make safety related decisions. Yet, these individuals perceived their opinions and questions were heard, and perceived they were progressively being allowed more autonomy. These individuals were classified in Table 6 as expressing autonomy even though their confines of performance were expressed as much narrower than their coworkers'.

\subsubsection{Support for safety from coworkers}

Coworker support for safety has been identified by several authors as an important dimension of perceptions of safety and safety climate (Törner, 2011; Zohar, 2000; Meliá, Mearns, Silva, \& Lima, 2008; Neal \& Griffin, 2006). The process of internalizing any given value is facilitated by coworker support (Gagne \& Deci, 2005: 333). As with autonomy, all 
the informants described their coworkers as supportive of safety, both of their own safety at the individual level and at the group level.

I always looked up to my peers. They would give me a hard time but it was all fun. They showed me right from wrong. They did not want to see anyone get hurt. Firefighter

I can't even begin to describe the amount of love and support that everyone at [my company] threw my way. It was just a fantastic group of people to have gone through such a horrible situation [a negative event] with.

Guide

The partner relationship is critical. If your partner, who was there with you and saw the same things and witnessed the same experiences, is not supportive of your reactions, that can be very detrimental because different people have different reactions based on all that other stuff that came up beforehand.

Paramedic

\subsubsection{Internalization versus externalization of safety}

Internalized safety does not necessarily exclude possibly concurrent external regulation ${ }^{22}$. Externalized motivation in this sample group was often attached to fear of administrative consequences - doing something (typically breaking a rule) that management would not condone or would penalize. The sole firefighter who expressed primarily external regulation based their safety motivation on preserving their social identity in their work crew:

... reputation is huge and generally speaking [...] reputations are pretty accurate. [You protect your reputation] by not screwing up. You screw up and people know. Firefighter

\footnotetext{
${ }^{22} 81 \%$ of the informants expressed primarily internalized regulation of safety; $17 \%$ indicated a role for both internalized and external control of safety behaviour, and $2 \%$ relied primarily upon external regulation to motivate their safety performance. This is detailed in Table $6 \mathrm{~b}$.
} 
As a specific example of the process of internalizing safety, the river guide group interview discussion often returned to the topic of the company's safety system. The company owner provided clear expectations on how the guides are to run the rapids (i.e. dictated with very specific paths through the rapids). The guides in the group interview believed this to be a distinguishing feature of their safety culture and debated the pros and cons of such a top-down approach. The discussion seemed open yet accepting of the context in which they worked.

The longer I've been here the more appreciation I get for [the safety system]. The real reasons why we do things, it's more the little things that I see that are just that much more obvious than I picked up on my first year.

Guide

There is a bit of struggle in terms of [the rigid safety], to me what wins out in that struggle is all of the other bonuses of working here.

Guide

I might have a slight disagreement with [the safety system], you don't fuel that. It is the game plan for the day. Again, it is a matter of balancing and fully respecting the choices that [the owner] made for the sake of safety. I'm not the one that signs the insurance paper at the end of the day. It's not my neck out on the line so much. Guide

These comments hint at a process of internalization. The comments imply an amount of sensemaking to come to terms with the safety requirements, and a slow appreciation and adoption. It also appears that adoption or internalizing is not without some internal tension and trade-offs. SDT principles are evident: competence and relatedness of safety. Autonomy is less present and is potentially the source of the internal conflict implied in the comments, yet there is still evidence of the informants choosing to 'buy in'. 
For the analysis of Proposition 3, seven individuals were removed from consideration as they did not meet the qualification of 'strong social identity' from Proposition 2. Even without a strong social identity, the two firefighters removed from analysis still expressed primarily internalized safety motivation. For the five paramedics removed, three expressed combined internalized and externalized safety motivation. Two paramedics expressed strictly externalized safety motivation. The paramedics explicitly discussed how fear motivated their safety performance; not fear of ability regarding patient care, but fear of breaking a rule and either being called out socially as incompetent or else fearing job reprisals.

What is better for the patient? It is what we can sort through on paper so that we don't end up getting our hand slapped or punished.

Paramedic

For the remaining SDT variables, three of the removed seven believed that they did not have autonomy regarding safety. For these three (paramedics), safety was about navigating conflicting rules. Two of the seven did not perceive coworker support for safety - the remaining five did. These sentiments were expressed in earlier themes.

In summary, Proposition 3 is supported. The data showed that when considering individuals who expressed strong social identity, they were more likely to internalize safety rather than externalize safety. When considering the variables that comprise SDT, a sense of competence for safety, autonomy to pursue safety, support from coworkers and relating safety performance to greater outcomes all were present and endorsed by this group. 


\subsection{THEME 6: SELF-CONCEPT WITH REGARDS TO SAFETY}

Theme 6 was targeted in advance to move beyond internalization of safety as a source of individual motivation to probe whether group-generated safety beliefs become adopted and incorporated into self-concept. Self-categorization theory predicts self-concept will become modified by membership in a group (Hogg \& Terry, 2000), and that individuals will behave in ways consistent with their self-concept (Shamir, 1991, Bandura, 1989).

Proposition 4 stated that given a strong social identity, the individual is more likely to incorporate group safety values into one's self-concept rather than externally regulated safety values ${ }^{23}$. All the informants either directly referred to safety in relation to their individual sense of self, or, when considering their interview commentary in its totality, provided clear indication that safety was tied to their self-concept. Therefore, Proposition 4 is supported. Support for the proposition is detailed in Table 7 following these results.

I do the job and I am good. I do it safely. Sometimes you have to go outside the parameters, too. I work safely and I train.

Firefighter

Self-categorization and self-concept theory identify the variables affecting self-concept adoption: including of safety in one's self-definition, and attaching personal meaning or social significance to safety values. These are examined in relation to Proposition 4.

\subsubsection{Safety in self-definition}

All but one of the informants expressed safety in their self-definition. One interview question specifically asked individuals to define safety performance. These responses

\footnotetext{
${ }^{23}$ Similar to Proposition 3, only the 48 individuals who expressed strong social identity are considered for analysis and assessing support for Proposition 4.
} 
were typically reflected outward to define a generic worker rather than themselves. Instead, self-definition was reliably expressed through responses to other questions such as: what motivates you to perform safely? Only one firefighter did not discuss how safety is attached to their self-definition or self-concept.

I don't want to perform my job badly. I will put pressure on myself. I don't feel it from others at this job, but I do feel it from myself.

Firefighter

It is not necessarily that I am safe, it is that I am capable. I think those two go handin-hand.

Firefighter

It's important for me to know that my coworkers can depend on me in those situations.

Guide

I want to be the type of partner that I would like to work with. You have to be able to trust your partner [and] count on your partner for your own safety. I want to be that partner.

Paramedic

\subsubsection{Personal meaning attached to safety}

Workers can attach personal meaning and social significance to their work when it is aligned with self-concept and when it is aligned with a group or community (Bunderson \& Thompson, 2009). Almost all the informants discussed or inferred safety practices or outcomes as personally meaningful above or beyond expectations of the job ${ }^{24}$ (Table 7).

I want to learn how to become a very efficient, good firefighter for myself, so my crewmembers have a lot of faith in me, and trust me $100 \%$, so when we are in a fire together, everyone feels comfortable. The more comfortable you feel, in that environment with your crew, the safer you feel when you are in a fire.

\footnotetext{
${ }^{24} 85 \%$ of informants discussed or inferred safety practices or outcomes as personally meaningful.
} 


\section{Firefighter}

I would be most upset at myself than anyone would be at me because if someone got hurt in my care I would be pretty choked. I would definitely think of what others think of me but I would be harder on myself than others would be on me. Guide

I know if my partner hurt their back because of something I did, because I was unsafe, I would never forgive myself so I think that's a big pressure behind it. Paramedic

\subsubsection{Safety adopted into self-concept}

All the informants expressing high social identity in Proposition 2 also expressed safety as incorporated into their self-identity and self-concept. This supports Proposition 4. According to self-categorization and self-concept theory, individual motivation can be interpreted as maintaining self-concept and fulfilling expectations associated with a social category (Shamir, 1991; Bandura, 1989). An individual's safety intentions will reflect safety values congruent with their self-concept, social group and role or profession.

It all depends [on balancing risk]; it is risk a lot to save a lot. If I am risking my life, it is going to be to save somebody else's life and it is going to be worth the risk. Firefighter

The seven informants removed from analysis of this proposition (as they did not express strong social identity in Proposition 2) split along sector lines. The two firefighters both expressed safety oriented self-concept regardless of the strong social identity qualifier. The five paramedics did not express safety as an aspect of their self-concept or selfdefinition.

In summary, Proposition 4 is supported. All the informants reflected safety as an aspect of their self-concept. Safety formed a portion of their work related self-definition, and 
most informants expressed that personal meaning was attached to their own perceptions regarding the importance of safety.

\subsubsection{Protecting self-concept}

A core aspect of self-concept theory states that staying consistent with one's self-concept becomes a need in and of itself, and a powerful trans-situational motivator (Shamir, 1991). A single safety oriented study endorsed this, finding pervasive social influence and social motivation to preserve one's self-concept (Mullen, 2004). Proposition 5 was targeted to seek such confirmation from this sample group, by proposing that an individuals' safety values and motivation will be directed towards protecting one's selfconcept and social identity.

This proposition was modestly supported, with qualification. Relative to the other proposition findings outlined above, Proposition 5 found lower support ${ }^{25}$ (Table 7). While firefighters and guides were interpreted as supporting this proposition, paramedics were not. Only approximately half of the paramedic informants expressed protecting one's selfconcept or social identity as a form of safety motivation.

Examples of comments provided by informants expressing safety motivated to protect self and/or social identity:

There is a lot of pressure on you there to drive [the fire truck to a call]. Sometimes you do make a wrong turn or something, and it happens to everyone. They may joke about it, but I have worked on other shifts where they are joking but it is almost not a joke, at the same time. So, there is a bit of a stress level there.

\footnotetext{
$2571 \%$ of firefighters, $86 \%$ of guides, and $45 \%$ of paramedics expressed some level of motivation to protect self-concept. When considering all informants $(N=55), 71 \%$ provided commentary that would support Proposition 5.
} 


\section{Firefighter}

Fear of looking bad? I think yes, and letting your crew down. Letting yourself and your crew down.

Firefighter

Well if you do screw up, like if something happens it's a serious negative affect. It's a black mark on your record, so it's trying to keep that perfect record. The fear almost drives you to be safe, if anything that's what we all really do.

Guide

I think it's every individual may feel differently working with someone they admire or think highly of, that might make you work harder so that you don't look stupid. Paramedic

You don't want to be known as 'that guy'.

Firefighter

During the data collection process, one particular incident proved insightful in interpreting safety motivation as protecting one's identity. In the ride along with a fire crew, the researcher shared a seat in the back of the truck cab racing to a call, sirens wailing. Two firefighters were getting suited into their gear, and one of the firefighters realized that they did not have their flash hood (a fireproof balaclava worn under the helmet protecting face and neck skin). The firefighter was observed urgently patting pockets, looking on the floor, and getting up to look under her seat. The firefighter quietly asked the crewmate in the back, 'I don't have my hood - do you have one?' The partner said no, but promptly broadcast to the rest of the crew in the truck 'Does anyone have an extra hood?' The driver in the front said he did. The firefighter who forgot their hood looked sheepish, even though the rest of the crew was highly focussed on getting to the fire and paid her no attention. The individual was interviewed later that day after the call: 
Q: From a safety perspective, what are the top three things you have to do every day?

A: Always check my [air] pack and mask are in good working condition and that they are there. If I'm driving check brakes, lights and a quick round about before you settle in. Check my gear and make sure I have everything I need.

Q: But you didn't have all your gear today? How does it happen that you check it but miss it?

A: On Saturday I was detailed to a different station. I always keep my flasher in my roof of my helmet but then if I'm at a call like a car accident where I'm not going to be using it I take it out [...]. So I guess I left it on a pump and then I packed up all my stuff, left in the morning and then today I don't have it, I just didn't look in my helmet.

Q: Is this a common trap of falling into a routine: Yes, I'm checking but I'm not?

A: It's funny because these [air] packs are new and l've always checked my pack, turn it on and do a check that everything is working. I am doing a full check but it is routine and I'd like to think that I would notice for my pack that's something off, not coming on or low l'd like to think I would and even though it's routine - ya I missed that one this morning so I'm happy [my coworker] had an extra one.

Q: So next shift are you going to do your gear check differently? Was that a close call for you?

A: No - if it was flames shooting whereas that was a lot of smoke [today]. I'm not really worried about burning my ears or singing my hair. If flames were shooting out as I'm going by the other pump operator and he wasn't using [his hood] I would have grabbed his.

This conversation exemplifies rationalizing an error so that it does not threaten one's selfconcept of safety competence or social standing. Checking PPE is the firefighter mantra, yet when this individual was proven to have failed in this task, it was not an issue, as given the outcome of the situation it did not matter - that gear was not needed. Further, the 
next interview question asked the same firefighter about balancing personal safety with getting the job done:

My personal safety there [only] is so much you can do before you go in; making sure you have all your gear and everything is working.

This was answered without a hint of recognizing the incongruity between earlier actions and present words. This firefighter, further in the interview, discussed the importance of reputation and not making mistakes; "when you screw up, people know," the firefighter said. A separate firefighter implied that appearing to be safe is more important than protecting one's own health.

You want to show the guys that you know what you are doing because this is your job. I want my skills to shine through what I am doing. You do not want to mess up on a call.

Firefighter

Meeting the group imposed performance standards and appearing competent in regards to safety could be interpreted as protecting one's social identity in the group, and possibly to affirm one's self-concept to one's self. This idea gains elaboration in Theme 7 that follows.

In summary, Proposition 5 is modestly supported. Firefighters and guides provided examples of safety motivation directed towards protecting one's self-concept or social identity. Only half of paramedics provided commentary supporting this proposition, showing the proposition's presence but cannot be conclusively supported by the paramedic group. One firefighter in particular provided a salient example of rationalizing an error in order to preserve either one's sense of self-competency or social standing. 


\subsection{THEME 7: TRUST}

The theme of trust was ubiquitous in the interview discussions. Trust emerged unprompted: there were no questions directed towards this theme yet every single informant $(\mathrm{N}=55)$ brought up the role that trust plays in their dealing with safety. This was unexpected. Measured by word count, trust ranked 11th overall, behind many obvious anchor words such as 'safety'. In any single interview trust often came up from several perspectives, such as 'trusting others' or 'trusting that I am safe' or 'I want to be trusted'. The researcher came to regard the theme of trust as the glue that was holding all the other themes together. Trust appeared in conversations regarding social influence and equally in comments regarding internalizing safety.

Across all three sectors in this study, trusting one's coworkers was discussed as reducing uncertainty with regards to safety. Differentially, firefighters and paramedics discussed trust as enabling performance, while guides used trust as a proxy for measures of competence. There was also interesting commentary on working when there is not trust.

\subsubsection{Trust reduces uncertainty with regards to safety outcomes}

All three sectors provided commentary that by having trust in one's coworkers, one felt 'safer' going into uncertain situations. Likewise, a lack of trust created ambiguity that required attention to mitigate:

I know I am in a dangerous situation, but the people I am with, I trust a lot and it makes it easier, for me, to be in that situation.

Firefighter 
I have gone on overtime with [crews] that I had no idea who they were. Your senses are heightened versus going in with people you do know. I did not feel any less safe, but I was more cautious.

Firefighter

I had trust in my coworkers, when you're out on the river I trusted that if something happened to me they would be there and help me out or I wouldn't have to clean up for them all time, they knew what they were doing.

Guide

If you have a crappy guide it's almost like a liability on the trip, it's like 'Ok, I have to keep looking', it just takes away from everyone's trip because you have to constantly be worrying about this extra thing.

Guide

When you're working with someone you don't know you don't have that expectation. So it's not trust as much as expectations, if you know what to expect it's a lot easier to accomplish what you want to accomplish versus if you don't know what to expect you're always looking and asking Ok what did you do? What did you ask? How did you do that?

Paramedic

You are in the back of the truck and you look over and [because I don't trust my coworker, it's like] I'm by myself today. If anything happens I'm on my own and that is a horrible feeling.

Firefighter

These informants imply through these comments that certain cognitive attention was freed up when a level of trust is in place. Without trust, some attentional effort was required to watch not only oneself, but also one's coworkers.

\subsubsection{Trust enables performance}

Firefighters and paramedics primarily discussed trust in relation to how it enabled individual or group performance. While earlier themes in these results presented 
commentary regarding shared experience and norms as agreed upon values, trust comments add further depth to coworker influence with regards to safety:

Q: What does that trust enable? A: Well, you are going to do stuff that you would not normally do. It is like a handshake - you shake their hand and they keep their word. There is no question or doubt. That is where I am with the guys that I work with. I keep myself around the guys that I can trust.

Firefighter

Q: What does trust enable? A: Performance. If everyone is at the same level and we all trust each other, in terms of ability; attention to detail. It enables team performance.

Firefighter

Your team needs to have faith in you, like you do in them. Otherwise, you don't get your job done. You can't work for yourself; you have to be a team.

Firefighter

You trust your partner that they are going to step up and say something if you have missed something because you can't remember everything.

Paramedic

On a professional level it definitely affects their performance and paramedics know it. If you delegate to your partner to perform an assessment, if they miss something, that could affect you professionally and the patient. So there is a lot of trust there that you're going to do what you're going to do and you're going to do it with the quality that we both expect. I would say trust is tremendous.

Paramedic

From this perspective, trust enabled group performance as it provided capacity an individual did not have alone. These comments imply an understanding that interdependent tasks require a trust relationship in order to be used as a successful strategy to manage safety critical situations. 


\subsubsection{Trust as proof of competence}

River guides used trust more often to describe a desirable personal worth or trait. Trustworthiness seemed to be a proxy for ability or competence, as in 'If I am trusted then I must be competent in the eyes of my coworkers'. This further implied a level of social acceptance and social identity.

I would hope that [my coworkers] would look at me and say you know if I get into a situation, this is somebody I would like to have having my back.

Guide

I want them to think I have their back just as much as I believe they have mine. It's a team thing; it's huge that they think I'm good at being safe.

Guide

It's important for me to know that my coworkers can depend on me in those [critical] situations.

Guide

Through the lens of personal competence, two firefighters expressed similar sentiment:

You want them to trust you as much as you trust them. You are a team and that is how it is supposed to be. You do not want to be the weak link on the team or the unsafe link, which is probably worse.

Firefighter

My coworkers think that I am safe. I think, if they think that, they have confidence in me when I am working with them. I feel that about them, which I hope they feel about me. Otherwise, it would be stressful working with someone in this job, that you didn't have confidence in and performing the duties safely.

Firefighter

In summary, trust emerged in a number of varying capacities and could be construed as present in many of the themes presented above. Trust was discussed as reducing uncertainty, enabling performance, and as a measure of personal competence. 


\subsection{SUMMARY OF RESULTS}

What is the influence of coworkers upon the individual internalization of safety? These findings link the data collected from 55 interviews to the research question, and lay out the pieces to answer this question in the discussion section that follows. Seven themes emerged from the data: four that were targeted with guiding propositions, and three emergent themes of shared experience and trust. Workplace observation and sample comments in the preceding themes showed support for each proposition and that proposition's underpinning theoretical variables. Viewed as a whole, the group of propositions and the logic that informed them was supported in this data, with some qualification.

Theme 1 presented workplace observations and informant perspectives that described shared experience as the basis for coworker influence. The physical workplace and the social environment of each sector were described as having differing qualities.

Theme 2 described findings built around Proposition 1, and found that safety norms were an assembly of agreed upon safety values (see Table 3 for a summary of support for each proposition). Both descriptive and concertive norms were clearly recognized in the workplace. Concertive norms were discussed extensively by informants and were perceived as more influential. Evidence of concertive norms were present throughout the interviews and were evident in the findings of Propositions 2 through 5.

Theme 3 presented evidence of sensemaking as a means of dealing with ambiguity with regards to safety. Paramedics, in particular, deal with ambiguity more than the other two 
sectors studied, with multiple layers of authority and conflicting priorities. This theme emerged from the data as it was not targeted in advance.

Theme 4 described findings built around Proposition 2, and found that most individuals expressed strong social identity with their work group. Paramedics were the exception, with only half of those informants expressing strong social identity with their work group. As a sector, paramedics were found to not support this proposition while firefighters and river guides did. Evidence of strong social identity was evident when using 'we' over ' $\mathrm{l}$ ' in discussing safety performance, relating task interdependence and relating a shared history of group success.

Almost all the informants described their safety orientation as internalized in Theme 5, forming strong support for Proposition 3. There was complete agreement that safety was autonomously pursued, and that there was social support for safety. The majority of informants believed that they were competent in regards to safety performance, and to a lesser extent related their safety priorities to greater conceptual outcomes. All of these variables comprise the core elements of SDT, found to be present in the data. This proposition was analysed with a smaller sample: only those that expressed strong social identity in Proposition 2 were considered $(n=48)$, as Proposition 3 built upon Proposition 2. 


\section{Table 3: Summary of support per proposition}

\begin{tabular}{|c|c|c|c|c|}
\hline & $\begin{array}{l}\text { Responses } \\
\text { supporting } \\
\text { (aggregated) }\end{array}$ & Firefighters & Guides & Paramedics \\
\hline $\begin{array}{l}\text { P1: Norms as agreed upon } \\
\text { safety values }(\mathrm{N}=55)\end{array}$ & $\begin{array}{c}51 \\
(93 \%) \\
\end{array}$ & $\begin{array}{c}28 \\
(100 \%) \\
\end{array}$ & $\begin{array}{c}16 \\
(100 \%) \\
\end{array}$ & $\begin{array}{c}7 \\
(64 \%) \\
\end{array}$ \\
\hline $\begin{array}{l}\text { P2: Individuals have high } \\
\text { social identity with group } \\
(\mathrm{N}=55)\end{array}$ & $\begin{array}{c}48 \\
(87 \%)\end{array}$ & $\begin{array}{c}26 \\
(93 \%)\end{array}$ & $\begin{array}{c}16 \\
(100 \%)\end{array}$ & $\begin{array}{c}6 \\
(55 \%)\end{array}$ \\
\hline $\begin{array}{l}\text { P3: Internalize safety over } \\
\text { externalize }(n=48)\end{array}$ & $\begin{array}{c}46 \\
(96 \%)\end{array}$ & $\begin{array}{c}26 \\
(100 \%)\end{array}$ & $\begin{array}{c}15 \\
(94 \%)\end{array}$ & $\begin{array}{c}5 \\
(83 \%)\end{array}$ \\
\hline $\begin{array}{l}\text { P4: Safety adopted into self- } \\
\text { concept }(n=48)\end{array}$ & $\begin{array}{c}48 \\
(100 \%)\end{array}$ & $\begin{array}{c}26 \\
(100 \%)\end{array}$ & $\begin{array}{c}16 \\
(100 \%)\end{array}$ & $\begin{array}{c}6 \\
(100 \%)\end{array}$ \\
\hline $\begin{array}{l}\text { P5: safety motivation to } \\
\text { protect self and social } \\
\text { identity }(\mathrm{N}=55)\end{array}$ & $\begin{array}{c}39 \\
(71 \%) \\
N=55\end{array}$ & $\begin{array}{c}20 \\
(71 \%) \\
n=28\end{array}$ & $\begin{array}{c}14 \\
(86 \%) \\
n=16\end{array}$ & $\begin{array}{c}5 \\
(45 \%) \\
n=11\end{array}$ \\
\hline $\begin{array}{l}\text { P5: safety motivation to } \\
\text { protect self and social } \\
\text { identity (considering only } \\
\text { those with strong social } \\
\text { identity } n=48 \text { ) }\end{array}$ & $\begin{array}{c}36 \\
(75 \%) \\
n=48\end{array}$ & $\begin{array}{c}19 \\
(73 \%) \\
n=26\end{array}$ & $\begin{array}{c}14 \\
(86 \%) \\
n=16\end{array}$ & $\begin{array}{c}3 \\
(50 \%) \\
n=6\end{array}$ \\
\hline
\end{tabular}

Utilizing the reduced sample group, Proposition 4 found complete support in that informants expressed safety as adopted into their self-concept. Safety formed a portion of the individuals' self-definition and expressed personal meaning attached to their safety performance and safety values, as aligned with self-categorization theory and with selfconcept theory.

Theme 6 described findings built around Proposition 5, which projected that safety performance would be oriented around preserving one's self-concept or social identity.

Firefighters and river guides supported this proposition. As with Proposition 2, 
paramedics were an exception. This is not surprising given Proposition 2 results, which found only half of the paramedics relating a strong social identity. Only half of the paramedics expressed preserving self-concept as a motivating factor.

The final theme, trust (Theme 7), was an unexpected, strongly represented theme. All informants discussed the presence of trust either as a part of social influence, or as a part of internalizing safety or self-concept. The implications of the emergence of this theme will be discussed in the discussion section that follows.

\subsection{SUPPORT FOR GUIDING PROPOSITIONS}

As an addendum to the expected and emergent themes provided above, this section details support for the propositions that guided this study. Separately describing how much a proposition was discussed provides a means by which to assess endorsement of that proposition. Miles and colleagues (2014) counting of qualitative data displays an overall view in support of what the results narrative explained in detail.

\subsubsection{Proposition 1}

93\% of informants discussed or inferred that coworker safety norms represented agreed upon group safety values. This equates to 51 of the 55 informants (Table 4). All the firefighters and river guides endorsed this proposition, but only $64 \%$ of the paramedics recognized safety norms as agreed upon group values. Concertive norms were more prevalent in the interview comments than were descriptive norms. 


\begin{tabular}{|c|c|c|c|c|}
\hline & $\begin{array}{l}\text { Responses } \\
\text { supporting } \\
\text { (aggregated) } \\
\mathrm{N}=55\end{array}$ & $\begin{array}{l}\text { Firefighters } \\
n=28\end{array}$ & $\begin{array}{l}\text { Guides } \\
n=16\end{array}$ & $\begin{array}{l}\text { Paramedics } \\
\mathrm{n}=11\end{array}$ \\
\hline $\begin{array}{l}\text { Safety norms } \\
\text { present in the } \\
\text { workplace }\end{array}$ & 54 (98\%) & 28 (100\%) & 16 (100\%) & 10 (91\%) \\
\hline $\begin{array}{l}\text { Descriptive norms } \\
\text { re safety }\end{array}$ & 49 (89\%) & 28 (100\%) & $14(88 \%)$ & 7 (64\%) \\
\hline $\begin{array}{l}\text { Concertive norms } \\
\text { re safety }\end{array}$ & 51 (93\%) & 25 (89\%) & $16(100 \%)$ & 10 (91\%) \\
\hline $\begin{array}{l}\text { P1: Norms as } \\
\text { agreed upon } \\
\text { safety values }\end{array}$ & 51 (93\%) & 28 (100\%) & 16 (100\%) & 7 (64\%) \\
\hline
\end{tabular}

\subsubsection{Proposition 2}

$87 \%$ of informants related a high safety oriented social identity with their work group. $100 \%$ of river guides, $93 \%$ of firefighters but only $55 \%$ of paramedics expressed high safety social identity (Table 5). The theoretical variables contributing to this proposition saw similar distributions of support, with a notable exception of paramedics, who strongly recognized task interdependence, yet showed little support for the associated social identity variables. 
Table 5: Proposition 2: Individuals working in safety critical or highly safety focused groups will have high safety oriented social identity with the group

\begin{tabular}{|l|c|c|c|c|}
\hline & $\begin{array}{l}\text { Responses } \\
\text { supporting } \\
\text { (aggregated) } \\
\text { N=55 }\end{array}$ & $\begin{array}{l}\text { Firefighters } \\
\mathrm{n}=28\end{array}$ & $\begin{array}{l}\text { Guides } \\
\mathrm{n}=16\end{array}$ & $\begin{array}{l}\text { Paramedics } \\
\mathrm{n}=11\end{array}$ \\
\hline $\begin{array}{l}\text { Speak primarily } \\
\text { with 'We' rather } \\
\text { than 'l' }\end{array}$ & $45(82 \%)$ & $25(89 \%)$ & $16(100 \%)$ & $4(36 \%)$ \\
\hline $\begin{array}{l}\text { Reference to task } \\
\text { interdependence }\end{array}$ & $51(93 \%)$ & $25(89 \%)$ & $16(100 \%)$ & $10(91 \%)$ \\
\hline $\begin{array}{l}\text { Reference to } \\
\text { history of shared } \\
\text { success }\end{array}$ & $42(76 \%)$ & $21(75 \%)$ & $16(100 \%)$ & $5(45 \%)$ \\
\hline $\begin{array}{l}\text { P2: Individuals } \\
\text { have high social } \\
\text { identity with } \\
\text { group }\end{array}$ & $48(87 \%)$ & $26(93 \%)$ & $16(100 \%)$ & $6(55 \%)$ \\
\hline
\end{tabular}

Responses were coded ranging from 'Not present' to 'Strong endorsement'. With regards to Proposition 2, guides provided exclusively conclusive and strong endorsement. Firefighters varied from weak to strong, while paramedics ranged from not present to conclusive, with no informant expressing strong endorsement of safety oriented social identity (Table 5b).

Table 5b: Proportion of individuals who have high safety social identity with group

\begin{tabular}{|l|c|c|c|c|}
\hline & Not present & $\begin{array}{l}\text { Low support or } \\
\text { reference to }\end{array}$ & $\begin{array}{l}\text { Yes conclusive } \\
\text { support }\end{array}$ & $\begin{array}{l}\text { Strong } \\
\text { endorsement }\end{array}$ \\
\hline $\begin{array}{l}\text { Firefighters } \\
\mathrm{n}=28\end{array}$ & 0 & $2(7 \%)$ & $21(75 \%)$ & $5(18 \%)$ \\
\hline $\begin{array}{l}\text { Guides } \\
\mathrm{n}=16\end{array}$ & 0 & 0 & $15(94 \%)$ & $1(6 \%)$ \\
\hline $\begin{array}{l}\text { Paramedics } \\
\mathrm{n}=11\end{array}$ & $2(18 \%)$ & $3(27 \%)$ & $6(55 \%)$ & 0 \\
\hline
\end{tabular}




\subsubsection{Proposition 3}

Given that 48 of the 55 informants expressed strong social identity with regards to safety and their group (Proposition 2), and as Proposition 3 builds upon or refines Proposition 2, only those 48 individuals supporting Proposition 2 were considered in evaluating support for Proposition 3. This includes six paramedics who expressed high safety social identity, even though as a group they were found to have low support for Proposition 2.

Of the 48 informants considered, 46 expressed internalized safety motivation over externalized motivation (Table 6). $100 \%$ of firefighters, $94 \%$ of guides and $83 \%$ of paramedics expressed this sentiment. All sectors believed that their coworkers supported safety. The SDT variables or competence, relatedness and autonomy were evident in the informants' commentary. 
Table 6: Proposition 3: Given a strong social identity, the individual is more likely to adopt and internalize coworker safety values rather than externally regulate safety values

\begin{tabular}{|l|l|l|l|l|}
\hline & $\begin{array}{l}\text { Responses } \\
\text { supporting } \\
\text { (aggregated) } \\
n=48\end{array}$ & $\begin{array}{l}\text { Firefighters } \\
\mathrm{n}=26\end{array}$ & $\begin{array}{l}\text { Guides } \\
\mathrm{n}=16\end{array}$ & $\begin{array}{l}\text { Paramedics } \\
\mathrm{n}=6\end{array}$ \\
\hline $\begin{array}{l}\text { Believe } \\
\text { competent } \\
\text { re: safety }\end{array}$ & $46(96 \%)$ & $26(100 \%)$ & $14(88 \%)$ & $6(100 \%)$ \\
\hline $\begin{array}{l}\text { Relates safety to } \\
\text { larger conceptual } \\
\text { outcomes }\end{array}$ & $41(85 \%)$ & $20(77 \%)$ & $16(100 \%)$ & $5(83 \%)$ \\
\hline $\begin{array}{l}\text { Safety } \\
\text { autonomously } \\
\text { pursued }\end{array}$ & $48(100 \%)$ & $26(100 \%)$ & $16(100 \%)$ & $6(100 \%)$ \\
\hline $\begin{array}{l}\text { Support for safety } \\
\text { from coworkers }\end{array}$ & $48(100 \%)$ & $26(100 \%)$ & $16(100 \%)$ & $6(100 \%)$ \\
\hline $\begin{array}{l}\text { P3: Internalize } \\
\text { safety over } \\
\text { externalize }\end{array}$ & $46(96 \%)$ & $26(100 \%)$ & $15(94 \%)$ & $5(83 \%)$ \\
\hline
\end{tabular}

Data display Table $6 \mathrm{~b}$ shows that $81 \%$ of the informants expressed primarily internalized regulation of safety; $17 \%$ indicated a role for both internalized and external control of safety behaviour, and $2 \%$ relied primarily upon external regulation to motivate their safety performance. 


\section{Table 6b: Evidence of Internalization versus Externalization of Safety}

\begin{tabular}{|l|c|c|c|}
\hline $\begin{array}{l}\text { Individuals expressing } \\
\text { primarily: }\end{array}$ & $\begin{array}{l}\text { Internal } \\
\text { regulation of } \\
\text { safety }\end{array}$ & $\begin{array}{l}\text { Both internal \& } \\
\text { external regulation } \\
\text { of safety }\end{array}$ & $\begin{array}{l}\text { External } \\
\text { regulation of } \\
\text { safety }\end{array}$ \\
\hline Aggregated $(n=48)$ & $39(81 \%)$ & $8(17 \%)$ & $1(2 \%)$ \\
\hline Firefighters $(n=26)$ & $22(85 \%)$ & $3(11 \%)$ & $1(4 \%)$ \\
\hline Guides $(n=16)$ & $12(75 \%)$ & $4(25 \%)$ & 0 \\
\hline Paramedics $(n=6)$ & $5(83 \%)$ & $1(17 \%)$ & 0 \\
\hline & &
\end{tabular}

\subsubsection{Proposition 4}

As with the previous proposition, only the 48 individuals who expressed high safety oriented social identity were considered for analysis in Proposition 4, as it builds upon Proposition 2 and 3. All 48 informants reflected safety as adopted into their self-concept. There was almost unanimous expression of safety as a part of ones' self-definition and that personal meaning was attached to safety (Table 7).

Table 7: Proposition 4: Given a strong social identity, the individual is more likely to incorporate group safety values into one's self-concept rather than externally regulated safety values

\begin{tabular}{|l|l|l|l|l|}
\hline & $\begin{array}{l}\text { Responses } \\
\text { supporting } \\
\text { (aggregated) } \\
\mathrm{n}=48\end{array}$ & $\begin{array}{l}\text { Firefighters } \\
\mathrm{n}=26\end{array}$ & $\begin{array}{l}\text { Guides } \\
\mathrm{n}=16\end{array}$ & $\begin{array}{l}\text { Paramedics } \\
\mathrm{n}=6\end{array}$ \\
\hline $\begin{array}{l}\text { Safety in self- } \\
\text { definition }\end{array}$ & $47(98 \%)$ & $25(96 \%)$ & $16(100 \%)$ & $6(100 \%)$ \\
\hline $\begin{array}{l}\text { Personal meaning } \\
\text { attached to safety }\end{array}$ & $41(85 \%)$ & $22(85 \%)$ & $14(88 \%)$ & $5(83 \%)$ \\
\hline $\begin{array}{l}\text { P4: Safety } \\
\text { adopted into self- } \\
\text { concept }\end{array}$ & $48(100 \%)$ & $26(100 \%)$ & $16(100 \%)$ & $6(100 \%)$ \\
\hline
\end{tabular}




\subsubsection{Proposition 5}

Relative to the other proposition findings outlined above, Proposition 5 found lower support. When considering all informants $(N=55), 71 \%$ provided commentary that safety motivation was directed towards protecting one's self-concept and social identity (Table 8). By sector, $71 \%$ of firefighters, $86 \%$ of guides, and $45 \%$ of paramedics expressed some level of motivation to protect self-concept. When considering only those informants who expressed strong social identity in Proposition $2(n=48)$, overall support goes up slightly to $75 \%$, distributed similarly at $73 \%$ of firefighters, $86 \%$ of guides, and $50 \%$ of paramedics. The strong social identity qualifier had little effect on this proposition's findings. While firefighters and guides were interpreted as supporting this proposition, paramedics were not. Only approximately half of the paramedic informants expressed protecting one's selfconcept or social identity as a form of safety motivation.

\section{Table 8: Proposition 5: An individuals' safety values and motivation will be directed} towards protecting one's self-concept and social identity

\begin{tabular}{|l|c|c|c|c|}
\hline & $\begin{array}{l}\text { Responses } \\
\text { supporting } \\
\text { (aggregated) }\end{array}$ & Firefighters & Guides & Paramedics \\
\hline $\begin{array}{l}\text { P5: safety motivation to } \\
\text { protect self and social } \\
\text { identity (considering all } \\
\text { informants } \mathrm{N}=55)\end{array}$ & $\begin{array}{c}39(71 \%) \\
\mathrm{N}=55\end{array}$ & $\begin{array}{c}20(71 \%) \\
\mathrm{n}=28\end{array}$ & $\begin{array}{c}14(86 \%) \\
\mathrm{n}=16\end{array}$ & $\begin{array}{c}5(45 \%) \\
\mathrm{n}=11\end{array}$ \\
\hline $\begin{array}{l}\text { P5: safety motivation to } \\
\text { protect self and social } \\
\text { identity (considering only } \\
\text { those with strong social } \\
\text { identity } \mathrm{n}=48)\end{array}$ & $\begin{array}{c}36(75 \%) \\
\mathrm{n}=48\end{array}$ & $\begin{array}{c}19(73 \%) \\
\mathrm{n}=26\end{array}$ & $\begin{array}{c}14(86 \%) \\
\mathrm{n}=16\end{array}$ & $\begin{array}{c}3(50 \%) \\
\mathrm{n}=6\end{array}$ \\
\hline
\end{tabular}


Overall, the first four propositions found strong support with the firefighters and guides. Paramedics provided partial support for certain propositions (Proposition 2) and full support for others (Proposition 4). The last proposition was expressed less with this group of informants, however with enough endorsement to consider the proposition valid and supported. The discussion section that follows will consider the implications of these findings. 


\section{DISCUSSION}

This study explored coworker influence upon safety values, evidence of which emerged in the interview data. Targeted and emergent themes were presented in the results section. Variables relevant to social influence theories such social identity theory and selfdetermination theory were evident in the findings. These theories are as-yet underutilized in safety studies, and can help to explain the findings from the present study.

Aspects of the results deserve further discussion. What is the influence of coworkers upon the individual internalization of safety? Safety was defined in the introduction as freedom from harm. The initial conceptualization of this study assumed safety in the physical sense, as in how coworkers influence an individual's understanding of their role in protecting patients, clients or the public from the hazards and potential harm inherent in their work. What emerged in the findings, and is expanded upon below, was a uniform belief that physical hazards were manageable and a secondary concern. Safety emerged as a socially significant set of behaviours that entrenched one in the work group, affirmed one's competency, and anchored one's sense of working identity. Safety at its most concrete level was about not getting hurt. Safety at its more meaningful level was about the potential harm from finding one's self on the outside of the work group and the internal or psychological implications of such exposure. This discussion explores this more meaningful interpretation of coworker influence upon safety. 
When considering this study in its totality, some key insights emerge to address the research question. At the group level of influence, the effect of social bonding upon safety culture development was poignant. Coworkers also played a significant role in managing ambiguity inherent in these safety critical sectors. Coworkers played an affirming role in validating an individual's sense of competence. Additionally, the role of storytelling and safety folklore seemed to be a vehicle of safety culture transmission and was a means by which group sensemaking could come to terms with inherent ambiguities such as role conflict. Lastly, dissonance was evident throughout, with some indications of individual strategies to reduce its effect, including internalizing safety. Each of these key learnings is discussed in turn.

This research examined coworker influence upon the internalization of safety beliefs at the individual level of analysis, but focused upon the (group level) influence of coworkers. The scope of this discussion is limited to theorizing on this social aspect, focusing on group or coworker influence. The psychology of individual internalization is deemed outside the scope of this discussion, as it was secondary within the framing of the research question guiding this study. As executed here, the psychology of internalization likely aligns with the safety psychology literature outlined in the literature review, however the specific variables relevant to individual internalization (such as differences in personality or locus of control) were by intention not addressed in the research design. This discussion section will theorize upon the research question of coworker influence upon individual internalization of safety. 


\subsection{THE ROLE OF SOCIAL BONDING}

The role of social bonding in establishing group level safety culture was immediately obvious as the interview process unfolded. The sense of cohesiveness of the firefighting crews and in the river guide group interview were palpable. That sense was also conspicuously absent from the paramedic base visits. The firefighters and guides related to each other with a sense of familiarity that seemed to enable conversations regarding safety priorities and shared beliefs - both in a group setting and individually. With paramedics there was no such agreement, and even in some cases there was reluctance to discuss a 'group' belief regarding safety. Social bonding seemed to enable shared understanding and agreed upon standards of safety performance.

Safety culture critic Perrow (1999) mused that, within an organization, there are safety 'cultures' (plural). This was evident in this study. Observing five separate crews in the urban fire service showed five variations on group culture. These differences were always subtle, such as the emphasis on driving or seatbelt safety versus getting to the scene as fast as possible, or differences in the balance between being task focused versus keeping one's head up and situational awareness. Some of this variation would reasonably be attributed to group leadership (i.e. the captain's influence) but in four of the five crews the captain was the newest member of the group (captains moved between crews more frequently than the crew itself was reorganized). During individual interviews, captains acquiesced to the social bond that existed before they arrived in the crew, and certain captains saw their role as serving the crew rather than leading it. For these reasons, the 
differences in group culture are attributed primarily to the dynamics and history of the group itself, rather than the leadership.

The context of safety climate and coworker influence seemed to be important. The constraints inherent in the paramedic dyad relationship seemed to inhibit the development of a group level shared understanding of safety. Wilkins and Ouchi (1983) identified the contextual factors conducive to developing a 'clan culture'. A 'clan', the authors defined, is equivalent to a specific local work culture with particular qualities, namely a high level of member goal congruence and a shared paradigm directing collective interests. Clan culture is argued to be a better mechanism to manage complex work with high uncertainty, as the alignment of individual and group interests manages itself in the proper direction of performance ${ }^{26}$. Factors conducive to clan culture are a stable work group with a shared history, regular and high interaction among members, and lack of alternatives in directing work. By these criteria, the paramedic sector lacks the required context to develop a 'clan', while firefighters and whitewater guides align with all factors. Even for full-time paramedics with a dedicated partner, interaction with other members is low (given the dyad structure) and paramedics find themselves overseen by several alternative and conflicting bureaucracies. Given this, paramedicine is not expected to self-manage like firefighting crews and guides. The existence of a clan criteria implies group level shared understanding and coworker influence.

${ }^{26}$ Clan culture is actively being developed in the information technology literature. 
The paramedics' situation does raise the question as to whether paramedic teams failed to come to agreement or if such agreement is not possible in a rotating, two person, parttime employment context. Given the additional variables confounding paramedics' understanding of safety performance expectations, it is likely not possible to draw rigid conclusions. The paramedic work context was not structured to promote coworker influence. What was clear in the findings was that fewer paramedics internalized safety, and there was less consistency in internalized understanding compared to firefighters or whitewater guides. Contextual factors apparently play a role in producing strong or weak group cultures and by extension safety cultures.

Time was evident as a factor in coworker influence, as indicated in clan criteria above. Time together in a team setting allowed for shared experience as a basis for shared understanding. The whitewater guides discussed how trust and confidence in team performance grew with time. Paramedics with full-time, dedicated partners experienced very different safety contexts than their part-time counterparts. One paramedic commented that their sector is built upon a foundation of individuality: paramedics are trained in college and learn and are tested individually. Firefighters and river guides train and are tested in a team setting based on more of an apprenticeship model. It seems like these early contextual factors and social bonding could influence a persistent understanding of one's obligation to one's coworkers.

What is not clear is if the group level cultures that were observed aligned with the greater organizational cultures in which they were embedded. It was beyond the scope of this 
study to investigate group or individual alignment within hierarchical safety climate or culture. Potentially, the group cultures that were observed may be replicas of the organizational culture which housed them, and the group consensus on performance standards could be parroting the 'company line'. If this was indeed the case (evidence in the findings indicate otherwise), this study's results are still relevant. As indicated above, it was not the content of the safety cultures that was under study, but the influence of coworkers in the individual internalizing of said cultural values.

The lateral relationships examined in this study point to significantly more complexity at the coworker and group level than the dominant hierarchical safety paradigms recognize. Safety culture literature leaves unspecified how organizational priorities are hierarchically transferred to the individual (if they are at all...). One is left with the impression that such transference is automatic and without cost. This group level analysis points to internalizing safety via a number of more complex coworker mechanisms; such as shared sensemaking and ambiguity reduction, social bonding leading to social identification and self-concept tied to the group, and proof of competence and identity via trust. Coworker influence also offers external pressure to buy in, to not be the weak link, and to not make errors, as all of which could jeopardize one's social or professional status. The adoption of group (or organizational) safety priorities is not without cost.

\subsection{DEALING WITH AMBIGUITY}

Much of individual safety behaviour, this study found, was directed towards ambiguity reduction. Individuals from all three sectors expressed safety as 'this is all I can do to exert 
control over an uncontrolled situation'. The nature of the work in these safety critical sectors, and the reason these sectors were sampled for study, was the uncertainty that surrounds even error-free performance. Certain motivation theories discuss the universality of 'uncertainty reduction' in human behaviour (Festinger, 1954, Alderfer, 1969, McClelland, 1961), and the emerging safety oriented social influence research (covered in the literature review) identified uncertainty reduction as a key social function of coworkers. Uncertainty, for this sample, took the form of ambiguity with regards to safety expectations.

Ambiguity was ubiquitous for the informants in this study, and the primary cited referents to aid in individual sensemaking were one's coworkers. Much of the shared agreement work teams established with regards to safety performance was about managing ambiguity inherent in their work. As discussed in the results section, sensemaking regarding role conflict, conflicting priorities, and unclear authority were all influenced by coworker discussion or interaction.

Firefighters and whitewater guides, as individuals, expressed understanding of the ambiguity in their roles consistent with other individuals in their sectors. In other words, group consensus was related through individual narratives, and these narratives were more or less uniformly aligned. These two groups had strong social bonds and shared experience to rely upon for sensemaking regarding ambiguity.

The fundamental role conflict these two sectors experienced (firefighters expected to put 'safety first' but put themselves at risk to save others; guides to take clients into 
hazardous rapids and then protect their safety once there) was discussed at an individual level, but those conversations often included the language of 'we'. It was common to hear "we've come to think of it as risk a lot to save a lot", or "we don't run that in high water", indicating social identity was attached to such understanding and reflected some shared belief. Within each individual fire crew there were side-handed indications of a range of personal positions on safety first versus save others. One informant directed the researcher to an online video of a speech by a New York City fire chief, which essentially said 'safety has gone too far, we need to get in there and save lives'. Note that the informant himself did not indicate this position directly, but did so indirectly by endorsing a speaker that (at the time of the interview) was unknown to the researcher. Most every crew also had a 'Mr. Safety'. This was the actual term used to identify the most safety conscious member of the crew. This badge was worn with pride by Mr. Safety, but was somewhat disparaging when used by others. This range of personal opinions leaves one to speculate if these differences in safety priority were more semantic than apparent, or represented only narrow differences in interpretation of what would otherwise be a consistent group position. These individual differences, for firefighters, may not matter: at a major call a chief dictates firefighting tactics and the captain supervises his crew to execute. The strong situation hypothesis is at play here (Mischel, 1977), in that there may not be much room for variation in safety performance, regardless of individual beliefs ${ }^{27}$.

\footnotetext{
${ }^{27}$ Aligned with the strong situation hypothesis, daily chores at the fire station allowed for variation in beliefs on accomplishing tasks (a weak situation). One Mr. Safety commented on the unnecessary risks some crew members take just to clean the second story windows, "hanging upside down by a rope".
} 
Realistically, the exact same fire call could be interpreted by both Mr. Safety and the risk taker as being perfectly aligned with their individual views.

Whitewater guides operationalized ambiguity regarding role conflict differently. The senior guides all shared anecdotes of client injuries (or in one case a death) related to the risks inherent in the activity. These anecdotes were typically related with appropriate gravity, but typically fell short of taking direct responsibility for an error that created the injury. Failing in this balance between exposure and protection was not discussed in the interviews even though informants offered hints - an example of struggling with fundamental role conflict. Most guides did discuss 'reading their crew', which was a euphemism for assessing how much risk to expose them to. The 'company line' down a rapid would, in most cases, be the maximum allowable risk (i.e. how close to get to the big waves or ledges), with options for safer lines available by staying away from these hazards. In this sense, each rapid presented a medium-strength situation, where the guide could decide (either for themselves or on behalf of their crew) to 'go big' or 'stay dry'. In-field observations of the whitewater guides in action evidenced some minor variation in routes chosen. The expectation from the trip leader and the general social setting was to 'go big'. Runs through rapids that generated big splashes got the most cheers from the passengers involved and the nearby rafts and guides watching their progress. Guides were visibly proud and displayed big smiles or other overt signs of triumph when they successfully delivered a big ride. Less successful rides, such as having a passenger fall out in a rapid and requiring rescue, lacked the same social characteristics. The guide would keep their eyes down rather than seek endorsement from their peers as 
they would after a good run. Success or shame was judged on a rapid by rapid basis. The ability to execute the company line was a performance goal for the guide, regardless of the passengers. More interestingly, the guide with a timid crew that stayed away from all hazards would finish the day personally dissatisfied, even though they operated in the best interest of their passengers. Role conflict here emerged between self-concept regarding competency in the rapids and meeting the client's needs.

The paramedic field experienced a different form of role conflict. Paramedic scope of practice has been expanding in recent years, with higher standards of care expected, more options for pre-ambulatory treatment, and unique to this jurisdiction, the paramedic service was expanding to cover shortfalls in rural EMS, such as doing remote access calls and search and rescue (roles not covered by urban EMS). The changing role of the paramedic created ambiguity as to what is meant to be a paramedic. This was expressed as problematic for some, and not acknowledged by others.

Part-time paramedics, sharing less social bond, less shared understanding, and more individual sensemaking, experienced persistent and inhibiting ambiguity. Social influence did not emerge as significantly offsetting this ambiguity. Indeed, paramedics expressed ambiguity hierarchically with overlapping authority above them, lateral ambiguity with no formal dyad structure and rotating partners, plus complex decision making and an unpredictable environment with every call. This environment has direct implications for patient care, as a default paramedic response is to 'turtle', as one informant put it - to defend oneself by withdrawing and doing the minimum required. One paramedic referred 
to himself as a glorified taxi service, implying that his hands were tied by conflicting policy and limited autonomy. This generates a different type of role conflict: paramedics are trained and inculcated to care for the patient, but the parameters of the job make it exceedingly difficult to execute this basic directive. The alternatives then, as presented by the informants, are to either internalize patient care and break whatever policy lies in one's way, or else distance oneself from patient outcomes by externalizing safety performance. Both options deal with ambiguity inherent in the job by creating alternate ambiguities.

Cognitive stress points were surprisingly similar between the firefighters and paramedics: responding to a call knowing only a few of the variables to expect. Launching into the unknown was dealt with very differently between the two groups, however, with firefighters assuming the worst (the $10 \%$ of calls that are critical) and paramedics assuming typicality (the $90 \%$ mundane). The difference in energy levels was striking. Firefighters exit full tilt. Paramedics exit reluctantly. From the simple measure of exit speed, the paramedic response is construed as a withdrawal response to cognitive (and likely emotional) burden. The researcher observed a decidedly resentful 'here-we-goagain' response upon the sounding of the medical call chime. Of significance is that paramedics in this study spend almost their full shift responding to back to back calls. Of the five fire stations visited, only one of them would regularly spend most of their shift in response mode; the other 4 were medium to low volume stations. The cognitive and emotional energy requirements of the paramedic work day would be exhausting, producing an understandable defensive response to call outs. This is a very different role 
conflict from the discussion above, with what is perhaps reluctance or resentment towards the very duty one is hired for (if motivation is externalized) or has adopted (if internalized). This would create difficult dissonance to reconcile, with links to literature on emotional labour and burn out (Brotheridge \& Grandey, 2002), and post-traumatic stress (Regehr \& Millar, 2007). This latter condition is a newly recognized hazard within the paramedic profession, although one without structured management intervention.

Unlike the other two sectors, whitewater guides uniformly expressed respect for their organization's management. The small organization and hands-on involvement of the owner lowered ambiguity with clear expectations and an articulated safety system. Conversely, it was typical of firefighters and paramedics to be critical of their employer and management ${ }^{28}$. Firefighters were critical of prescribed policy that added ambiguity rather than reduced it: rules that cannot be followed because "that's not how it works". On the other hand, paramedics' shift supervisors were often bypassed for advice. One paramedic explained that when looking for advice on a call she often phoned colleagues on their time off, as she valued their opinion more than the supervisor's. With conflicting authority above them, some paramedics relied upon coworker interpretation of ambiguity - although this was not consistent across the paramedic sector (some utilized supervisors, and others dealt with it themselves). Firefighters and paramedics perceived that their management structure complicated the uncertainty inherent in their job, rather than clarified it.

\footnotetext{
${ }^{28}$ Recall that firefighter crew captains were not considered management, as captains are involved in front line delivery of safety with their crews.
} 
At face value, it seems that having strong institutional regulations overseeing one's sector should reduce ambiguity. This was not the case. Paramedics, as heavily regulated, experienced persisting ambiguity. Whitewater guides, with no regulatory oversight, expressed relatively little ambiguity with regards to performance expectations. This raises the question of the role and legitimacy of such regulatory oversight, if it is inhibiting safety understanding rather than enhancing it.

This question of legitimacy of oversight was questioned in early systems thinking safety research (Rasmussen, 1997) and has become operationalized in the 'Just Culture' movement, which aims to move oversight from punishing errors to improving systems $\left(\right.$ Reason, 1997) ${ }^{29}$. The small-scale whitewater sector has what these large regulationbased industries are trying to develop: a focus on front line operations and decision making in the field, a focus on dealing with errors commensurate with preventing errors, and recognizing a direct trade-off between safety and efficiency (i.e. safe operations are slower and have direct costs associated with it). The paramedics' working environment would not meet the criteria of a just culture with individuals operating under a fear of reprisals; the guide sector would be closer to the just culture intentions, being development and learning oriented (likely inadvertently due to this sector's reliance on young, novice guides). Firefighting lands between these two. This study's findings provide a group or individual level understanding of what is so far, a very high level discussion (in that field) regarding regulation.

\footnotetext{
${ }^{29}$ Aviation and health care are both (attempting) to embrace Just Culture - both heavily regulated sectors.
} 
Policy, when at conflict with operations (as was evident for firefighters and paramedics), added to ambiguity, but the nature of that ambiguity needs to be questioned. The seatbelt mandate, at one time written off by firefighters as "that's not the way it works", was slowly gaining adoption. Clearly, it could work that way, by adding what one informant estimated to be at most 10 seconds to response times. The original interpretation of the debate was better considered as "that's not the way we've done things". Social bonding, clan culture, and negotiated safety norms reduce uncertainty but at the same time can create entrenchment and resistance to change. The ambiguity generated through regulatory change, for firefighters, was about change, rather than regulation. A new process of group sensemaking and performance consensus was required to come to terms with the new requirements. Contrast this to paramedics - who were also grappling with a new seatbelt mandate - for whom it has proven (according to the informants) impossible to perform hands-on care in the back of the ambulance while buckled into a seat. Paramedics would need the interior of their vehicles to be redesigned if they are to fulfill both patient care and personal safety. This is a different form of ambiguity than firefighters' historical practice being at odds with current data-driven safety protocols.

\subsection{SAFETY FOLKLORE AND GROUP SENSEMAKING}

It was entertaining to listen to the stories of the informants - they all had them. Each sector had anchor stories that served as safety folklore: repeated tales that informed safety beliefs. The fact that these same stories came up repeatedly leads one to consider 
their social significance. At the most superficial level, these stories were examples of injunctive norms, what one 'ought' to do (or 'ought-not' to do). Considered in context, each sectors' stories also inadvertently served as group sensemaking regarding the substantial ambiguities facing each sector.

Firefighters referred to one of two stories: several years earlier four firefighters were forced to jump from an upper story window down to the ground. As a result, the service adopted a 'rapid rappel' PPE belt for such situations. Three of the five fire crews discussed this story and how part of their pre-fire routine now involves ensuring their belt is accessible. After several years, no one had yet to have need for the belt. The story provided poignancy to a low probability risk that turned into a safety routine. Firefighters referred to a second, more predominant safety folklore tale of a crew saving a victim by jumping into an icy river. This story likely persisted as folklore as it exemplified one of the core ambiguities of firefighting: safety first versus putting one's self at risk to save others. This is a hero story, which very directly relates the group or professional expectation regarding the balance of safety to front line workers. This was likely only the most recent iteration of this story, which would have replaced a similar hero story of a previous generation. The researcher observed this story as laterally shared, but it is not known if the story persisted hierarchically as well (i.e. if chief or superintendent level messaging included this folktale and therefore value statement). While firefighters recognized role ambiguity, their own folklore provided clear indications of the proper or expected balance. From this perspective, safety folklore becomes concertive (Barker, 1993), and storytelling becomes a means of indirectly relating group level safety priorities. This story 
becomes a form of group sensemaking: firefighters are expected to put themselves at risk.

Several firefighters were interviewed who had similar 'non-normal' rescue experiences in their tenure. Paradoxically, for these individuals, they did not consider theirs hero stories. For the other firefighters, the river rescue story reinforced the 'risk a lot to save a lot' paradigm. For firefighters involved in such rescues, these individuals discussed the fine line between 'hero' and getting into trouble. When the outcome was positive, the crew were heralded as heroes. Yet individually there was acknowledgement of the extreme (and uncomfortable) physical risk and that if things turned out negatively how the crew would have been maligned (and penalized!). For certain situations and for certain individuals, there was a sense that their rescue actions were over the line - the opposite interpretation of individuals not directly involved. For individuals involved in such rescues, the story was more complex than its folklore iteration, and did not resolve the ambiguity that others used it to reduce.

Paramedic safety folklore told a tale, from sometime in the past (the date of which no one could recall), of a team being first on scene where a man fell through the ice on a lake. One of the paramedics ran to a neighboring boarded up cottage, grabbed ${ }^{30}$ a canoe, and used it to traverse the thin ice and rescue the victim. The paramedic was awarded a variety of commendations, including the Order of Canada, depending on who told the story. For a small number of individuals this story created a hero narrative sorely lacking

\footnotetext{
30 "Grabbed" was used by those for whom this story re-enforced the hero narrative. "Stole" was used by those who saw this story as problematic scope creep.
} 
in the typical, mundane, and thankless working reality. For a larger number of individuals, this story exemplified the role conflict they felt and the scope creep experienced in recent years. 'So is this now expected?' asked one paramedic in relation to the story. The paramedic service had recently invested in specialty rescue training, all-terrain vehicles, and search drones. While many viewed the canoe rescue story through the lens of ' wouldn't do that', there was now doubt and ambiguity around expected performance. As this rescue training was often directed towards full-time paramedics, this further complicated the part-time paramedics' understanding of their role. This folklore, unlike for firefighters, was used to exemplify ambiguity in paramedicine rather than clarify it. From a sensemaking perspective, this specific story allowed paramedics to crystalize their ambiguity and share it amongst themselves, rather than resolve it.

River guides, too, shared stories. As the guide sample covered individuals across a variety of companies and geographies, no single event tied them together. However, most guides told a similar story, and it often involved some epic big run, a flipped raft, and a heroic and efficient saving of the passengers mid rapid. The story often included mild pejorative towards the clients, highlighting their helplessness and therefore the value of the guide. This story, too, had undertones of both the hero and role conflict, tending towards legitimizing the guide role (ignoring that the guide created the problem in the first place) and projecting desired competency.

Why did these stories persist? The act of storytelling itself had social significance for these groups, and clan culture relies upon storytelling as a means of relating shared history. 
That these specific stories persisted can be considered by what they have in common: each story took place on the boundaries of 'normal' and 'non-normal', they included a hero narrative, they expressed an undercurrent of sector-specific ambiguity, and they were success oriented. While it is highly unlikely a firefighter will again need to jump from an upper story window or a paramedic will need to 'steal' a canoe, these events highlight the boundary of normal operations and become a sensemaking vehicle. No one individual can experience all the variations of non-normal, so these stories take on folklore status to represent all possibilities of low probability/high consequence events (and are cognitively engaging!). The shared aspect of the hero narrative placed the teller at the centre of the story, with all manner of psychological and philosophical implications, as the hero quest is the original folk tale genre. The hero tale imbued importance and legitimacy to one's understanding of one's work. As safety critical operations carry inherent risk and some random variability, the hero tale reinforced what may not always be clear: agency, competency, and an element of control. The ties to these particular stories likely persist because they address (via simile) underlying issues relevant to the group. Lastly, these are success stories. The researcher heard very few stories of failure, and those that were mentioned were alluded to rather than detailed. The success stories were likely a means of dealing with cognitive dissonance inherent in this work (considered in detail in a section below).

One of the themes that emerged in the findings was the role that coworkers played in assisting with individual sensemaking and ambiguity reduction. Storytelling and safety folklore seemed to play an important group level of sensemaking role, becoming a vehicle 
by which to discuss individual levels of perception by a less personalized means. Weick (2010), in theorizing on group level sensemaking, argued that there is a fundamental shift in sensemaking from an individual internal process to a different group level social categorizing. Weick proposed that "if people want to share their cognitive structures, those structures have to take on a particular form" (Weick, 2010: 541). This represents a shift from perception based sensemaking to category based knowledge. At a group level, a "shared logic of exchange" is required (Allard-Poesi, 2005: 173) - a communication mechanism to facilitate this group sensemaking (Balogun \& Johnson, 2004). This safety folklore serves this purpose. The stories become a means by which to express one's individual sensemaking without personal or intimate exposure. Relating or commenting upon the actions or choices of others is a relatively safe means by which to discuss ambiguous topics such as role conflict and establish group level consensus on the interpretation of such events.

When observed in a group setting, it was the senior guides and firefighters who told the stories. Obviously, years on the job created more stories to tell, but storytelling was observed as a means by which senior workers controlled the safety message. It could be speculated that storytelling is one of the means by which senior firefighters 'push' rather than receive social pressure - a point identified in the results section. Tied to group sensemaking literature, this would be termed 'sensegiving'.

The act of storytelling itself took on significance. Whitewater guide culture is one of storytelling, often around a campfire in the evenings. A fire captain lamented the 
diminishing role of storytelling in firefighting culture, with a slow move away from apprenticeship training and more towards technical schooling. One firefighter commented that sometimes one hears stories so often around the kitchen table that "it seems like I was there". This, perhaps, is the point of the storytelling. To place oneself in an unexperienced, difficult situation is a means of rehearsal and schema building. Storytelling becomes concertive by dictating the group safety performance expectations.

Individuals carried their own stories as well. One firefighter, in his early days, inhaled a lung full of smoke. "I coughed for three days" he said, and then explained that his air mask was now his main PPE priority. When asked if he shared his story and safety priorities with others, he responded "definitely". A whitewater guide related a similar near miss with a stack of boats tumbling off a trailer - it is now his prevention priority that he forced upon other guides he worked with. These individual experiences developed personal safety priorities and internalized certain aspects of safe performance, perspectives they pushed onto their coworkers.

Both the safety folklore and personal experiences were used to define the boundary between normal and non-normal events, reinforced ones' importance in the face of uncertainty, and provided a group vehicle by which to discuss and conceptualize the ambiguity inherent in safety critical work. Storytelling and folklore became a means of group sensemaking and dictating safety performance expectations. 


\subsection{SAFETY AS SOCIALLY SIGNIFICANT AND VALIDATING COMPETENCY}

Along with much of safety behaviour being directed towards ambiguity reduction, an equal part was directed towards appearing competent. For those interviewed in this study, to be considered safe by one's coworkers was to be considered competent. To be considered competent served both as social status and as personal validation.

Appearing to be safe was more important than protecting one's health. In only a couple of instances did an informant express moments of concern for their physical safety. Appearing unsafe, on the other hand, was fatal from a social perspective. "You don't want to be known as "that guy'" said one firefighter. Whitewater guides often referred to making sure one was not the "weak link". Firefighters all claimed to know who the unsafe workers were, and all dealt with them by either staying clear of them or else staying close to them - to keep an eye on them. However, none of the firefighters interviewed claimed they could possibly be 'that guy'. This is an example of defending ones' self-concept and of social identity in action: to be a firefighter is to be a member of a crew; a crew must trust each other; one gains crew trust because one is competent. With this logic, how could one be a firefighter and not be competent? The alternative, to admit to one's self that perhaps I'm 'that guy', is to admit one is untrustworthy and therefore not part of the crew. One, therefore, is not a firefighter. Psychological defenses regarding self-concept would make this admission existentially difficult given social bonding, clan culture and social identity. Coworkers play a critical role in affirming one's working identity. 
Work identity is attached to producing a desirable impression, something that is more difficult in work that lacks objective measures of competence or output (Alvesson, 2001) such as safety work, which must instead rely upon coworker endorsement. What is external endorsement of competency may serve as internal affirmation of identity (Pratt, Rockman, \& Kaufmann, 2006). Modern work on identity and in-group definition separates self-definition from self-investment (Leach et al., 2008). While self-definition is simpler and relies upon voluntary self-categorization with a group, self-investment requires group endorsement. Trust from coworkers encourages investment in the group; the investment in the group enables social identity and self-identifying with the group's values (Masson \& Fritsche, 2014).

Appearing safe extended to the firefighter mantra of checking PPE. Even when one informant was caught out as deficient in this respect, the individual justified their actions to preserve an appearance of competency. Interestingly, each firefighter was completely thorough by their own standards when it came to checking PPE, yet in private believed that some others on their crew were not thorough enough. Clearly, coworkers were watching each other on simple (but socially significant) safety routines and went on to establish judgements regarding individual safety competency. Safety routine took on social significance. River guides discussed the same safety appearances of "head on a swivel" and watching for the safety of other rafts. Failing to appear diligent in this task coloured one's socially perceived safety abilities, and thereby jeopardized one's appearance of competency. Festinger's (1954) social comparison theory would argue that coworkers covertly watching each other is a means of comparatively assessing one's own 
abilities or status. For what appears to be a binary activity (i.e. Is my gear ready to go? Yes or no), there are potentially social gradations of performance with little bearing on the final outcome (i.e. I can't believe coworker X does X). Speculatively, this social comparison activity could contribute to individual appraisals of the crew pecking order, which for firefighters, at least, is espoused as simply seniority based.

Appearing to take time and care checking gear may represent a social commitment to one's coworker safety, and thereby commitment to the group. Being thorough is a show of being trustworthy. Being trustworthy fulfills both the individual need for competency validation and one's social safety obligation to the group. Cutting PPE checks short could be seen as disrespecting one's coworkers. Modern work on higher order goals (a.k.a. modern needs theory) would argue that there is an ever-present orientation towards meeting one's social obligations and need for social acceptance (Barrick, Mount, \& Li, 2013, Gagne \& Deci, 2005). This raises an interesting question regarding the 'standard' to which coworkers are covertly judged. Is it judged against one's own standard (i.e. 'He is not as thorough as ( $\mathrm{am}$ ') or by some group established safety performance norm? Is the individual letting the group down, or is it a more individual assessment relative to the observer's abilities?

Unsafe workers were on the outside of the social group because they did not meet the agreed upon safety standards. One informant initially stated "We work hard not to make errors", which was interpreted by the researcher as having high internal standards. The same informant later said "everybody knows who makes the mistakes...". Error reduction 
evidently also served the purpose of preserving one's place in the social and professional hierarchy.

Having the trust of one's coworkers seemed critical to the individual assessing their own competency. Whitewater guides, in particular, seemed to rely upon coworkers to validate their sense of competency (more than the other two sectors studied). In a sector with no external regulation nor empirical measures of competency, coworker trust equated to validation of competency: 'if my coworkers trust me then I must be good'. This validation of competency, it could be speculated, was about endorsing self-definition and selfinvestment in the group. Paramedics, including the part-timers with rotating partners, expressed the same desire to be seen as trustworthy. Trustworthiness emerged as a form of currency regarding safety. One's reputation, across all three sectors studied, preceded the individual in their work settings. Safety became currency in the form of circulated reputation. Further, this sense of individual competency seemed (for some) to always be at risk, and had a need to continually be affirmed. One whitewater guide discussed how getting a "black mark" on one's reputation created fear-driven safety performance. This is an externalized form of safety motivation, as it is driven by social acceptance, and hints at a deep insecurity with one's work and social identity. This insecurity may stem from the ambiguities inherent in the job, where the actual definition of the role (and therefore sense of working self) is complex, uncertain, or unclear. SDT (Gagne and Deci, 2005) anchors competency as a basic human need and motivational goal. 
In the language of this current discussion, an individual's sense of competency may be their first defense in dealing with the ambiguity in their work, but more existentially, coworker endorsement of competency may also anchor basic work identity.

\subsection{DISSONANCE AND INTERNALIZATION}

Within these safety interviews, contradictions emerged at every turn. Firefighters entered burning buildings, guides dealt with rafts flipped over in powerful rapids and (in one case) a paramedic dealt with a sword wielding patient. Yet no one claimed to feel unsafe in their job. A firefighter will wear a seatbelt driving their own car but can justify not wearing one while speeding to a call in a fire truck. Paramedics work in teams of two but refused to recognize that one of them was the leader, even when paired with someone of higher or lower qualifications. Individuals apparently made all sorts of justifications for their individual behaviour in order to deal with the cognitive dissonance apparent in their narratives and inherent in their work.

All sectors dealt with an overbearing and unresolved existential dissonance. The 'safety first' edict is directly at odds with a public expectation of putting one's self at risk in order to fulfill the prescribed duty. After interviewing the informants, it could be generalized that their internalized safety hierarchy placed the duty to put one's self at risk as higher than (or "bigger" than) the safety-first message. Most would insist safety is second. This dissonance was exhibited in a variety of ways (discussed above).

In general, individuals were very satisfied with the safety systems in which they worked. Every firefighter claimed to trust their crew, but could point to other crews that were 
untrustworthy. River guides felt that their company was safe but other companies were not as diligent as theirs. If the primary form of dissonance in this study was the 'safety first versus put one's self at risk' role conflict, a second prominent form of dissonance would be the paradox of feeling safe in an unsafe environment. Feeling safe in one's unsafe work environment may be based on direct experience: the fact is, a worker can go many days on the job with no adverse effects. Individual experience of success could overshadow the substantial potential for injury that is present every day. Dr. J. MacCurdy (1943), who coined the term 'near miss' (in writing of survivor responses to World War II London bombings), recognized the sense of invulnerability that often accompanies seeing destruction all around but coming away unscathed. The safety critical workers in this study may have adopted a version of this invulnerability (i.e. feeling safe) in the face of continual exposure to risk. This could be an example of psychology's attribution error (taking personal credit for circumstance (Ross \& Nisbett, 1991)) and confirmation bias (explaining a situation with what one wants to be true (Reason, 2001)). Believing one's self as immune to the present hazards becomes a means of dealing with the cognitive dissonance of being unsafe all of the time, essentially by believing the opposite ${ }^{31}$. Safety folklore may play a role in reinforcing the 'feeling safe' position, by promoting agency, competency, and an element of control. The social messages of competency derived from coworkers reinforce this further.

\footnotetext{
${ }^{31} \mathrm{~A}$ concurrent aspect at play is likely self-selection. Those that could not 'cognitively navigate' the continual exposure to risk would self-select out of the job.
} 
From an observer's perspective, the informants' feeling safe in an unsafe environment takes on an element of denial. An example can be considered regarding this potential denial: trust in one's coworkers. As identified in the results, trust emerged unprompted and by every informant. Trust was uniformly recognized as a necessary ingredient for group and individual safety performance. 'You have to trust your coworker' was offered as safety mantra. Social identity theory predicts and explains the finding that firefighters and river guides claim to trust their own crew (the in-group), but could point to other crews or companies that are untrustworthy (the out-group). Yet, in individual conversation, many individuals hinted that they did not trust everyone they worked with. It was common to hear 'Yes I trust my partner but I double check everything they do'. Redundancy is a basic safety principle, but is that really trusting one's coworker? One firefighter related with distaste that another on his crew could not start a chainsaw, hinting this as an example of low competency and therefore low trustworthiness. A river guide related trusting his coworkers but every day felt the need to watch one lower skilled coworker carefully, waiting for an inevitable flipped raft that the informant would have to help clean up. The driving paramedic typically kept an ear open to ensure the partner was covering all required attending procedures. These indirect competency appraisals reflected lack of trust in the individual, beyond the convenient alignment with redundancy and double checks. This, despite the 'I have to trust my coworkers' mantra. Social-information processing theory might explain this, in that individuals adapt their attitude, beliefs (and eventual behaviour) to their social context (Salancik \& Pfeffer, 1978). By extension, if all social indicators maintain that one needs to trust one's 
coworkers, the individual "must either reject [these] judgments or assimilate them into his or her judgement. [...] the worker may want to agree with coworkers, if only verbally, to fit in. These repeated verbal agreements may eventually convince the worker himself," (Salancik \& Pfeffer, 1978: 229). Mandatory trust in one's coworkers may be a form of individual and group denial.

The reality for most safety critical tasks encompassed by the sectors under study here is that individuals operate in parallel rather than collaboratively. Guides are in charge of their own boat, paramedics split duties between attending and driving, and many aspects of firefighting are individually task focused. The production of safety requires multiple inputs, but each individual controls their own task independently. When things go right, coworkers do not need to be trusted. If something goes wrong, however, coworker support is crucial. Is this a source of dissonance, a denial of the unsafe work environment in order to avoid the difficult admission that not all coworkers can be trusted to perform if something goes wrong? Previous discussion surrounding trust inferred that individuals readily accepted it from coworkers as endorsement of their competency, but hint that they did not necessarily give it out as easily. If we assume that some individuals experienced even a modest amount of dissonance in the trust mantra versus actual confidence in and reliance upon their coworkers, then trust, used in this sense, might be very superficial. If this lack of trust exists within the work groups, and if there is an element of denial regarding feeling safe, this is likely because the alternative, feeling exposed within one's working safety system, is too difficult to cognitively navigate. 
Feeling unsafe would threaten the sense of self and individual work identity that these social structures work to prop up for each other.

The internalizing of safety motivation did not seem to come without additional aspects of dissonance. The whitewater guide group interview discussed that "buying in" to the safety system involved some trade-offs regarding choice and personal risk tolerance, and that those trade-offs were rationalized as justifiable by the individuals. Joining a fire crew involved giving up some sense of self and adopting the prescribed group identity. Recall the earlier exchange with the paramedic who broke all provincial guidelines by manipulating call severity in order to get directed to a better hospital. This safety behaviour, internalized and directed at receiving (what he perceived to be) the best care for the patient, comes with personal risk and (one has to assume) significant cognitive dissonance. The internalization of the higher order safety principles justified away or perhaps 'trumped' the dissonance of breaking policy and creating job risk. This was one example presented that individuals possessed an internalized safety values hierarchy. These were simply labeled by informants as "little things" and "big things". Little things could be sacrificed or broken in the name of pursuing big things. Paramedics uniformly discussed how to achieve the best patient care (a "big thing") almost always involved breaking provincial guidelines or policy (a "little thing"). The firefighting crew that went into the freezing river to save someone (a firefighters' highest value) did so knowing they could get into trouble, because the rules that they broke in doing so were all of them, including "the big ones". This internalized safety hierarchy may be evidence of a strategy to manage the cognitive dissonance inherent in these safety critical sectors. 
This raises a critical question: does the internalization of safety mitigate the cognitive dissonance inherent in these safety critical jobs? The balance of probabilities points to yes. By voluntarily internalizing safety, one gains all the social benefits that come with it: social acceptance in the work group, a sense of competency, and identity that creates agency and a sense of control. SDT argues the very reason one voluntarily adopts group values is to alleviate uncertainty and to fulfill basic needs of acceptance and competency. With the individual and social benefits obtained from internalizing safety, plus the employment of dissonance management strategies such as denial and internal attribution, an argument can be made that tips the ambiguity balance in the favour of the individual. Ambiguity can be managed or is in one's control.

For all of these reasons, it could be hypothesized that internalizing safety makes one more safe on the job. By adopting group safety values, one is empowered to act even when the correct action may be unclear. That empowerment may be to fulfill one's internalized duty, or merely to not appear incompetent, be the weak link, and thereby threaten one's social standing and internal sense of self. Even just going through the motions, Reason (1998) argues, can lead to internalized performance: "acting and doing [...] can lead to thinking and believing" (Reason, 1998, n.p.).

Can the opposite be true? Can cognitive dissonance hinder internalizing safety? The data in this study suggests this may be possible. Most every informant related some form of cognitive dissonance, yet the most internalized workers were the firefighters and whitewater guides. Strong social bonding and group sensemaking characterized these 
sectors. The paramedic sample confirms this with its opposite: this group was less internalized from a safety motivation perspective, and did not benefit from the same social factors as the other sectors, yet also confronted the greatest ambiguity. It cannot be concluded that cognitive dissonance was an interfering factor in internalizing safety, but the data here suggests a correlation between ambiguity, social support and internalizing safety.

\subsection{SUMMARY}

McCracken's (1988) long interview qualitative research method explains an analytic process, by which the researcher moves from the world as the informants see it to how the researcher now sees their world - from what was said to what is now known. While the informants saw norms, social identity, self-concept and trust in coworkers, the researcher found social bonding and group sensemaking as the primary means of mitigating inherent and overbearing ambiguity and cognitive dissonance. Safety took on

a social significance that directly related to one's sense of competency and sense of working identity.

Social bonding was found to be an important contextual factor that contributed to the development of a strong group safety culture. This strong social bond or group culture had a positive effect for firefighters, who were found to struggle with ambiguity regarding role conflict and balancing 'safety first versus save others'. Whitewater guides relied upon their social bonds as endorsement of individual competency. Paramedics, lacking this social structure, struggled with role conflict in caring for patients amid conflicting 
regulations that made such a goal difficult. The stories and safety folklore shared amongst a sector was viewed as reflecting fundamental uncertainties for that group. The stories became a vehicle for group sensemaking, which ultimately aided in individual sensemaking and coming to terms with inherent ambiguity.

Appearing safe and appearing competent were more important than protecting one's own health. Safety appearances played an important role in fulfilling group performance expectations and protecting one's social identity and individual sense of self. These appearances directly related to a thin sense of trust one granted to others and perceived to receive from coworkers. Safety was found to represent the potential harm from finding one's self on the outside of the work group and the internal or psychological implications of such exposure.

Lastly, dissonance was evident throughout this study. Expectations of 'safety first' and feelings of being safe in an unsafe environment were fraught with dissonance. The ambiguities discussed throughout such as role conflict and trust contributed further to dissonance. Mitigation strategies such as denial, a values hierarchy, and internalizing safety were all used to offset what would otherwise be inhibiting existential questions. 


\section{CONCLUSION}

What is the influence of coworkers upon individual internalization of safety? There is significant complexity at the coworker or group level regarding safety, more so than the predominant hierarchical safety climate paradigm includes.

This exploratory study operationalized group level safety climate to focus on coworker influence. The literature review assessed the breadth and depth of safety research at the organizational and at the individual level of analysis, and identified a significant gap that existed when considering group level influence. The coworker aspect only recently became a variable for academic study, with only a few papers exploring this perspective. Additional gaps were identified in the literature review regarding the presence of safety values and how organizational or group safety priorities become internalized at the individual level. Essentially, safety behaviour variables have been identified and organized, but the interactions and influences between them are just beginning to emerge.

This qualitative study explored the interaction between coworkers and their influence upon the individual internalizing safety. Particular themes emerged from the results: social safety norms manifest themselves as performance expectations; there was evidence of group sensemaking and coworkers assisting individual sensemaking with regards to managing the ambiguity inherent in these safety critical sectors; socially identifying with one's work group and incorporating safety into self-concept aided in the internalization of group safety priorities; and trust emerged as an enabling service that 
coworkers provided, and became the glue that held the various coworker influence and individual interactions together. From these themes, some key lessons emerged regarding coworker influence.

The social significance of safety and the role of trust in affirming competency and sense of working identity are the primary conclusions drawn from this study. Indeed, much of safety behaviour seems to be oriented around preserving one's place in the social setting, and in affirming one's sense of competency, agency, sense of control and work identity. An informant summed this up as: "You don't want to be known as 'that guy"”.

Social bonding proved to be a critical variable in assessing coworker influence for the three safety critical sectors studied. From this perspective, coworker influence did not prove consistent across occupational settings. Firefighters and whitewater guides had much in common, aligned with their levels of support for the various research propositions and providing similar narratives regarding social identity, the role of concertive norms, and internalizing safety. Paramedics expressed varied support for the role of coworker influence. A sharp distinction emerged between paramedics with fulltime partners and those on a part-time, rotating partner basis. Coworker influence, trust and safety internalization were more often expressed from those with full-time partners (who managed to grow a strong social bond), and much less so for those who worked part-time.

Storytelling and folklore emerged as a vehicle by which groups built shared understanding of performance expectations and were used to define the boundary between normal and 
non-normal situations. This ties to an additional key learning of the complexities that emerged in navigating ambiguity at both the group and individual level.

'Being safe' proved to have social significance beyond protecting one's own physical health. Simple daily actions such as checking personal protective equipment took on social significance and became a means by which one's ability to deliver on safety expectations - and by extension one's competence - was judged by peers. Trust emerged as a means by which individuals assessed their own competence, as the social validation was used as internal validation. This internalization was not without cost, as examples of cognitive dissonance appeared throughout the narratives provided by the informants.

What is the influence of coworkers upon individual internalization of safety? The findings of this study provide the following answer: through shared experience, safety folklore and sensemaking, a group establishes safety performance norms. Social bonding and trust allows for the individual to socially identify with their group and internalize group safety priorities and incorporate these into self-concept. Coworker social support and trust validated the individual's sense of competency. This sense of competency was the first defense in dealing with work ambiguity, but more existentially, coworker endorsement of competency also anchored basic work identity. Safety at its most concrete level was about not getting hurt. Safety at its more meaningful level was about the potential harm from finding one's self on the outside of the work group and the internal or psychological implications of such exposure. 


\subsection{CONTRIBUTION OF THIS STUDY TO THEORY}

This study operationalized group level safety climate to focus on coworker influence. By doing so, it found significantly more complexity at the group level than the existing safety climate paradigm considers. Findings regarding individual internalization of group safety beliefs indicate that underutilized social influence theories provide a valid means of explaining group values adoption. The major theoretical contribution of this research is a finding that coworker influence and social identity were an active influence upon internalizing safety motivation.

Supervision was originally interpreted as the group level within safety climate, based on Zohar's (2000) original rationalization that management plays the role of interpreting the organization's policies and priorities. The newer perspective re-interpreting coworkers as the group level uses the same proximal referent logic. This study found extensive lateral influence from coworkers. In at least two of the sectors, coworker influence was valued over management influence. In all cases, lateral relationships re-interpreted the hierarchical safety messages. This re-interpretation was based upon shared group experience and group sensemaking. There was clear evidence of safety as a social construction. These qualitative findings align with and provide depth to modern coworker influence quantitative safety studies.

The importance of coworker trust emerged as a key theme, a phenomenon yet to have any substantial presence in safety studies (at the group or individual level of analysis). The literature identified a coworker role of reducing ambiguity; trust emerged here as a 
(previously) unspecified mechanism by which uncertainty was reduced. Trust as proof of competence is a new theoretical perspective on individual safety motivation, one with significant complexity given how trust was differentially accepted from and offered to others. This finding offers depth to the generic 'coworker support' as enabling internalization of performance.

The coworker, with its new emergence in safety literature, has yet to have any theoretical structure. Three variables emerged from this study's findings that contribute to the theoretical understanding of coworker influence upon safety: group stability, group leadership, and social identity strength. Individuals in stable teams expressed more consistent internalization of safety than did those in part-time or rotating teams; structured team leadership was correlated to reduced ambiguity regarding performance expectations; individuals' strength of social identity with their work team was related to their internalization of safety beliefs. Other variables seem relevant in the coworker/individual setting, but these three had clear relationships in this qualitative exploratory setting.

Social influence theories provide a path by which individuals adopt coworker group safety beliefs. These social influence theories have yet to appear in safety literature. Social identity theory provided a valid means of explaining the individual's need to "buy in" and identify with their work group with regards to safety. This theoretical perspective was found applicable to socially intensive work crew settings, and less so when individuals work in 'weak' relationships such as part-time paramedics. 
Self-determination theory can be applied to explain a process by which individuals adopt group safety beliefs. Socially generated safety norms can become individually internalized, SDT explains, with a sense of competence, autonomy, relatedness, and coworker support. This was found to apply to the safety oriented social work environments in this study. This application of SDT is significant in that it details what safety culture and climate have yet to articulate - a mechanism or process by which external values become internalized. This study provided a qualitative link between coworkers and individual internalization, however SDT could potentially extend hierarchically as well as laterally. The role of shared experience, social bonding, and trust all offer depth to the application of SDT variables.

When considering coworker influence contribution to the greater field of motivation studies, this study's findings regarding social identification, internalizing group values, social bonding relevant to ambiguity and dissonance, and even socially validating competency all seem relevant beyond safety. This study can contribute to aspects of the greater motivation field with all of these findings.

\subsection{CONTRIBUTION OF THIS STUDY TO PRACTICE}

At a concrete level, this study's findings offer some clear direction to managing safety critical teams (and potentially to managing team performance in general) ${ }^{32}$ :

\footnotetext{
32 These points are derived from the contrast between successful firefighter and whitewater guides' social identity and internalization compared to the paramedic social experience.
} 
1. Keep teams together. Members of dedicated teams expressed stronger social identity and internalization of group beliefs. These individuals expressed less ambiguity with regards to the mechanics of dealing with safety critical situations. The findings from this study point to dedicated teams being as relevant to dyads as it is to seven person crews.

2. Trust is important for team function and individual performance. Shared experience and social bonding allows for consensus on group performance expectations. These expectations allow for social identity, uncertainty reduction and individual assessments of competency. 'Team-building' takes on new relevance when driven down to the individual level of internalizing motivation.

3. Assign a team leader. Lateral relationships are defined by their lack of hierarchy, however the findings from this study point to lack of structured leadership as cognitively taxing. Social groups intuitively recognize a leader; an explicit work structure that over-rides this fact adds another variable of ambiguity to already ambiguous work. The captain on the fire crew and trip leader on the river became social anchors around which a team built shared understanding. Less experienced workers looked to these social leads to negotiate uncertainty.

4. Train senior workers on how to mentor. The firefighters and guides both recognized a shift from being a receiver of social pressure to being a sender of social pressure. For firefighters, this seemed to be in the six to 10 years of experience range, while for guides it was in the three to five-year range. None of 
the work places studied provided training in the 'middle' years on leadership, team function, or mentoring.

5. Provide clear lines of authority. Hierarchical relationships need to be distinct; overlapping authority, such as in the paramedic sector, created significant ambiguity that detracted from individual internalization of motivation and of safety performance.

6. Policy needs to reduce uncertainty. Top down mandated policy or directives need to reduce uncertainty rather than contribute to it. The individuals in this study could point to several management decisions that complicated their personal safety or performance rather than enhanced it. Top down messaging needs to be consistent and oriented towards front line implementation.

Conceptually, organizational culture in this study provided background context and assumptions rarely directly addressed by the informants. This implies there was significant group level interpretations of safety performance. Clear and consistent organizational messaging would be required to influence these social constructions. The safety literature identifies variables of safety culture, such as two-way communication, worker input on procedures, and sensitivity to front line operations; all were visible and relevant in this study when comparing higher functioning teams to others.

As a take-away for managers from this study: individual safety motivation could potentially be enhanced by improving group bonding and social identity. 


\subsection{LIMITATIONS OF THIS RESEARCH}

There are several limitations to this research. This study was bound by a certain scope, so must "accept the inevitable limitations and dilemmas of our methods as constraints within which we must work..." (McGrath, 1981: 201). Interviews as employed in this research provided findings high on realism due to their contextual nature but low on generalizability to other settings. This lack of generalizability is recognized as a limitation to this study, but is accepted as the required trade off in the examination of an understudied phenomenon.

The researcher's frame of reference for this study is seen through the lens of a male of European descent, analyzing male dominated sectors in this study, and having specific work history in the adventure and whitewater guiding sector. It is recognized that such a frame of reference brings potential gender and racial bias, embedded in one's life and work history. Considerations for gender and race do not appear in safety studies literature at any level. The sectors under study are male dominated, primarily of European descent (although not exclusively), and as such the coworker influence phenomenon under study may incorporate gender and racial bias entrenched in those sectors. This study did not attempt to isolate bias within the studied coworker influence, but accepts as a limitation its potential presence in the findings.

The informants in this study represent just three of many safety critical industrial sectors. Findings from the sampled sectors may not transfer to others. Two of the sectors, firefighting and paramedicine, sampled individuals from a single service or employer (the 
urban fire service and the rural paramedic service). Findings on coworker influence from these sectors may include contextual factors that are specific to those organizations. The whitewater guide sample spanned a wide variety of employers and geographies, reducing this limitation for this group.

From a sample size perspective, for a qualitative study of this nature the overall sample of $\mathrm{N}=55$ is suitable. However, upon data analysis, the paramedic sample stratified between full time and part time informants. This essentially split that sector from $n=11$ to $n=4$ full-time and $n=7$ part-time. Understanding of paramedic coworker influence for each of these two subgroups would be enhanced with a greater sample size per subgroup.

This research did not compare coworker influence to supervisory or management influence, nor to organizational culture, for that matter. The interview focus was on the social setting, however, informants often expressed opinion on supervisory influence which contributed to the social influence findings generated with this study.

At the individual level, this study generated findings on the internalization of safety motivation and coworker influence. This study did not attempt to correlate informants' espoused safety motivation or beliefs against their actual safety behaviour or performance. This study also made no attempt to articulate actual group safety beliefs and their relative transference to the individual.

This study examined only one possible pathway and source of influence upon internalizing safety motivation. Intuitively, it seems that there would be several concurrent forms of 
influence upon any one individual. The literature review identified and mapped several factors regarding safety motivation. This study made no attempt to isolate the coworker influence from all others, other than to focus interview discussion in that direction.

Lastly, as a qualitative study, the coding, categorization of utterances, and assembling of themes were open to subjective interpretation and possible bias. The proposition guided research model utilized here both controls for and allows for bias. The propositions directed what the researcher was looking for, but also created bias through expectation. The researcher was conscious of such bias, and attempted, as per McCracken (1988), to maintain distance, rigour, and objective perspective throughout.

\subsection{IMPLICATIONS FOR FUTURE RESEARCH}

As an exploratory study looking to provide insight into an emerging coworker influence stream of study, this study's findings open several avenues worthy of further investigation. There are both specific questions remaining with this research project, and larger coworker influence implications.

An assumption in this study is that internally motivated safety behaviour will produce better safety outcomes for the individual and organization. This assumption is present in most every safety study, yet is rarely stated. This is a testable proposition which would back fill one of the key assumptions in the field.

A notable theme that emerged in this research was the presence of ambiguity. A further study examining how ambiguity relates to internalizing safety is warranted. Does 
ambiguity inhibit internalization or promote it? While ambiguity was ubiquitous in this study, no clear indication emerged as to its relationship to internalizing safety motivation. Trust emerged as a factor mitigating the cognitive effects of ambiguity. The shape and form of this relationship is wide open for further study.

Variables surrounding group or team structure are yet to be validated. Three variables emerged from this study which need further examination across a variety of team settings (group stability, group leadership, social identity strength). Further qualitative study in different settings or sectors would likely identify additional variables. Interaction effects may exist between these variables, opening an avenue to further study the complexity of coworker influence. Quantitative study could further validate and parse out the relationships between these variables. Likewise, the fire and paramedic sectors are unionized; even though the role of the union was not evident in the interview data, there is an opportunity to further examine the effect or lack of effect that a union plays in individual internalization of safety.

The additional factors generated by this study (trust, the role of social bonding, safety as socially significant, trust as proof of competence and dissonance) have all a stream of research behind them unrelated to safety. Pulling these constructs into the safety sector and developing dedicated research around them would add further complexity to coworker influence.

As indicated in the literature review, safety research (at the individual level of analysis) is organized around the paradigm of compliance versus participation. No such distinction 
emerged in this study's findings. This raises several intriguing questions: is the separation of compliance and participation a non-issue in safety critical work? There was some evidence of compliance discussion - seatbelts for firefighters and PPE for paramedics but safety participation, positioned as 'extra' in industrial safety, seemed to be the core element of these safety critical jobs. According to this study's informants, compliance was more often discretionary. This predominant paradigm may break down or not apply to safety critical sectors. Further examination of safety critical sectors and how individuals prioritize and segment understanding of their performance may offer insight and further complexity to the proximal and distal factors affecting safety performance. 


\section{BIBLIOGRAPHY}

Ajzen, I. (2001). The Theory of Planned Behavior. Organisational Behavior and Human Decision Processes 50, 179-211, 50, 179-211.

Alexander, M., Cox, S., \& Cheyne, A. (1995). The concept of safety culture within a UK offshore organisation. Understanding Risk Perception conference. Aberdeen: Robert Gordon University.

Al-Hemoud, A., \& Al-Asfoor, M. (2006). A Behavior Based Safety Approach at a Kuwait Research Institution. Journal of Safety Research , 37, Institution,' Journal of Safety Research, 37, 201-206.

Allaire, Y., \& Firsirotu, M. (1984). Theories of Organizational Culture. Organization Studies, 5, 193-226.

Alvesson, M. (2010). (POST-)POSITIVISM, SOCIAL CONSTRUCTIONISM, CRITICAL REALISM: THREE REFERENCE POINTS IN THE PHILOSOPHY OF SCIENCE . In M. Alvesson, \& K. Sköldberg, Reflexive Methodology; New Vistas for Qualitative Research Second Edition. Sage.

Alvesson, M. (2001). Knowledge work: Ambiguity, image and identity. Human relations, 54(7), 863-886.

Andriessen, J. (1978). Safe behaviour and safety motivation. Journal of Occupational Accidents , 363-376.

Antonsen, S. (2009). Safety culture and the issue of power. Safety Science, 47 (2), 183191.

Arnould, E., \& Price, L. (1993). River magic: Extraordinary experience and the extended service encounter. Journal of Consumer Research , 20, 24-45.

Attarian, A., \& Siderelis, C. (2013). Injuries in commercial whitewater rafting on the new and gauley rivers of west virginia. Wilderness \& Environmental Medicine , 24 (4), 309-314.

Baker, S., \& Edwards, R. (2012). How many qualitative interviews is enough? National Centre for Research Methods Review Paper 019.

Bandura, A. (1989). Human agency in social cognitive theory. American Psychologist , 44, 1175-1184.

Bandura, A. (1977). Social Learning Theory. Englewood Cliffs, NJ.: Prentice Hall. 
Bandura, A., \& Locke, E. (2003). Negative self-efficacy and goal effects revisited. Journal of Applied Psychology, 88, 87-99.

Bardi, A., \& Schwartz, S. (2003). Values and Behavior: Strength and Structure of Relations. PERSONALITY AND SOCIAL PSYCHOLOGY BULLETIN , 29 (10), 1207-1220.

Bargh, J., Gollwitzer, P., Lee-Chai, A., Barndollar, K., \& Trotschel, R. (2001). The automated will: noncouncious activation and pursuit of behavioural goals. Journal of Personality and Social Pscychology, 81, 1014-1027.

Barker, J. (1993). Tightening the iron cage: Concertive control in self-managing teams. Administrative Science Quarterly , 38, 408-437.

Barney, J. (1986). Organizational Culture: Can It Be a Source of Sustained Competitive Advantage? Academy of Management Review , 11 (3), 656-665.

Barrick, M., Mount, M., \& Judge, T. (2001). Personality and performance at the beginning of the new millennium: What do we know and where do we go next? International Journal of Selection and Assessment, 9, 9-30.

Barrick, M., Mount, M., \& Li, N. (2013). The theory of purposeful work behaviour: the role of personality, higher-order goals, adn job characteristics. Academy of Management Review, 38 (1), 132-153.

Barsade, S. (2002). The Ripple Effect: Emotional Contagion and Its Influence on Group Behavior. Administrative Science Quarterly , 47, 644-675.

Barton, M., \& Sutcliffe, K. (2009). Overcoming dysfunctional momentum: Organizational safety as a social achievement. Human Relations , 62 (9), 1327-1356.

Baumeister, R., \& Leary, M. (1997). Writing Narrative Literature Reviews. Review of General Psychology, 1 (3), 311-320.

Bentley, T., Page, S., \& Walker, L. (2004). The safety experience of New Zealand adventure tourism operators. Journal of travel medicine , 11 (5), 280-286.

Beus et al. (2010). Safety climate and injuries: an examination of theoretical and empirical relationships. Journal of Applied Psychology , 95, 713-727.

Beus, J., Dhanani, L., \& McCord, M. (2014). A Meta-Analysis of Personality and Workplace Safety: Addressing Unanswered Questions. Journal of Applied Psychology, no page specified. 
Blau, G. (1993). Operationalizing Direction and Level of Effort and Testing Their Relationships to Individual Job Performance. Organizational Behavior and Human Decision Processes , 55 (1), 152-170.

Blau, P. (1964). Exchange and power in social life. New York: John Wiley.

Brondino, M., Silva, S., \& Pasini, M. (2012). Multilevel approach to organizational and group safety climate and safety performance: Co-workers as the missing link. Safety Science, 50, 1847-1856.

Brotheridge, C. M., \& Grandey, A. A. (2002). Emotional labor and burnout: Comparing two perspectives of "people work". Journal of vocational behavior, 60(1), 17-39.

Brown, A. (2000). Making sense of inquiry sensemaking. Journal of Management Studies, 37, Studies 37, 45-75.

Brown, S., Lent, R., Telander, K., \& Tramayne, S. (2011). Social cognitive career theory, conscientiousness, and work performance: a meta-analytic path analysis. Journal of Vocational Behaviour, 79, 81-90.

Brown, W. E., Margolis, G., \& Levine, R. (2005). Peer evaluation of the professional behaviors of emergency medical technicians. Prehospital and disaster medicine, 20(02), 107-114.

Bunderson, J., \& Thompson, J. (2009). The Call of the Wild: Zookeepers, Callings, and the Double-edged Sword of Deeply Meaningful Work. Administrative Science Quarterly , 54, 32-57.

Burke, M., Sarpy, S., Tesluk, P., \& Smith-Crowe, K. (2002). General safety performance: A test of a grounded theoretical model. Personnel Psychology, 55, 429-457.

Burnkrant, R., \& Cousineau, A. (1975). Informational and normative social influence in buyer behaviour. Journal of Consumer Research , 2 (3), 206.

Cable, D., \& Parsons, C. (2001). Socialization tactics and person organization fit. Personnel Psychology, 54, 1-23.

Chiaburu, D. (2010). The social context of training: coworker, supervisor, or organizational support? INDUSTRIAL AND COMMERCIAL TRAINING , 42 (1), 53-56.

Chiaburu, D., \& Harrison, D. (2008). Do Peers Make the Place? Conceptual Synthesis and Meta-Analysis of Coworker Effects on Perceptions, Attitudes,OCBs, and Performance. Journal of Applied Psychology , 93 (5), 1082-1103. 
Choudry, R., Fang, D., \& Mohamed, S. (2007). The nature of safety culture: A survey of the state-of-the-art. Safety Science, 993-1012.

Christian, M., Bradley, J., Wallace, J., \& Burke, M. (2009). Workplace safety: a metaanalysis of the roles of person and situation factors. Journal of Applied Psychology , 94, 1103-1127.

Cialdini, R., \& Goldstein, N. (2004). Social influence: Compliance and conformity. Annual Review of Psychology, 55, 591-621.

Clarke, S. (2013). Safety leadership: a meta-analytic review of transformational and transactional leadership styles as antecedents of safety behaviour. Journal of Occupational and Organizational Psychology , 86, 22-49.

Clarke, S. (2006). The Relationship Between Safety Climate and Safety Performance: A Meta-analytic Review. Journal of Occupational Health Psychology, 11, 315-327.

Clarke, S., \& Robertson, I. (2008). An Examination of the Role of Personality in Work Accidents Using Meta-analysis. APPLIED PSYCHOLOGY: AN INTERNATIONAL REVIEW , 57, 94-108.

Clarke, S., \& Robertson, T. (2005). A meta-analytic review of the Big Five personality factors and accident involvement in occupational and non-occupational settings. Journal of Occupational and Organizational Psychology , 78, 355-376.

Colley, S., \& Neal, A. (2012). Automated text analysis to examine qualitative differences in safety schema among upper managers, supervisors and workers. Safety Science, 50 (9), 1775-1785.

Collie, R., Shapka, J., \& Perry, N. (2012). School climate and social-emotional learning: Predicting teacher stress, job satisfaction, and teaching efficacy. Journal of Educational Psychology , 104 (4), 1189-1204.

Cooper, J. (2011). Cognitive dissonance theory. Handbook of theories of social psychology , 1, 377-398.

Cooper, M. (2000). Towards a model of safety culture. Safety Science, 36, 111-136.

Cox, S., \& Cox, T. (1991). The structure of employee attitudes to safety: a European example. Work and Stress, 5 (2), 93-106.

Cropanzano, R., \& Mitchell, M. (2005). Social Exchange Theory: An Interdisciplinary Review. Journal of Management, 31 (6), 874-900. 
Cunliffe, A. (2010). Retelling Tales of the Field: In search of organzational ethnography 20 years on. Organizational Research Methods , 13 (2), 224-239.

Deal, T., \& Kennedy, A. (1982). Corporate Cultures. Reading: Addison-Wesley.

Denison, \& Mishra. (1995). Toward a Theory of Organizational Culture and Effectiveness. Organization Science , 204-23.

Denison, D. (1996). What is the difference between Organizational Culture and Organizational Climate; a Native's point of view on a decade of paradigm wars. Academy of Management Review , 21 (3), 619-654.

Denissen, J., \& Penke, L. (2008). Motivational individual reaction norms underlying the five factor model of personality: first steps towards a theory based conceptual framework. Journal of Research in Personality , 42, 1285-1302.

Díaz, R., \& Cabrera, D. (1997). Safety climate and attitude as evaluation measures of organizational safety. Accident Analysis \& Prevention , 29 (5), 643-650.

Dutton, J., Dukerich, J., \& Harquail, C. (1994). Organizational images and member identification. Administrative Science Quarterly , 43, 293-327.

Edmondson, A., \& McManus, S. (2007). METHODOLOGICAL FIT IN MANAGEMENT FIELD RESEARCH. Academy of Management Review , 32 (4), 1155-1179.

Ehrhart, M., \& Naumann, S. (2004). Organizational citizenship behavior in work groups: A group norms approach. Journal of Applied Psychology , 89, 960-974.

Eid, M., \& Diener, E. (1999). Intraindividual variability in affect: Reliability, validity, and personality correlates. Journal of Personality and Social Psychology, 76 (4), 662-676.

Eisenhardt, K. (1989). Building Theories from Case Study Research. Academy of Management Review, 14 (4), 532-550.

Ellemers, N., de Gilder, D., \& Haslam, S. (2004). Motivating individuals and groups at work: A social identity perspective on leadership and group performance. Acadamy of Management Review , 29 (3), 459-478.

Evans, J., \& Frankish, K. (. (2009). In two minds: Dual processes and beyond. Oxford: Oxford University Press.

Festinger, L. (1957). A Theory of Cognitive Dissonance. California: Stanford University Press. 
Festinger, L. (1954). A theory of social comparison processes. Human Relations, 7, 117140.

Fiske, S. (2003). Five core social motives, plus or minus five. In F. Z. Spencer, Motivated social perception: The Ontario symposium (Vol. 9, pp. 233-246). NJ: Erlbaum.

Fogarty, G., \& Shaw, A. (2010). Safety climate and the Theory of Planned Behavior: Towards the prediction of unsafe behavior. Accident Analysis and Prevention , 42, 14551459.

Ford, M., \& Tetrick, L. (2008). Safety motivation and human resource management in North America. The International Journal of Human Resource Management , 19 (8), 14721485.

Fugas, C., Melia, J., \& Silva, S. (2011). The "Is" and the "Ought": How Do Perceived Social Norms Influence Safety Behaviors at Work? Journal of Occupational Health Psychology, 16 (1), 67-79.

Fugas, C., Silva, S., \& Meliá, J. (2012). Another look at safety climate and safety behavior: Deepening the cognitive and social mediator mechanisms. Accident Analysis and Prevention , 45, 468-477.

Furnham, A., Eracleous, A., \& Chamorro-Premuzi, T. (2009). Personality, motivation and job satisfaction: Hertzberg meets the Big Five. Journal of Managerial Psychology , 24 (8), 765-779.

Gagne, M., \& Deci, L. (2005). Self-determination theory and work motivation. Journal of Organizational Behavior, 26, 331-362.

Garcia-Herraro, S., Mariscal, M., Gutierrez, J., \& Toca-Otero, A. (2013). Bayensian network analysis of safety culture and organizational culture in a nuclear power plant. Safety Science, 53, 82-95.

Gersick, C. (1988). Time and Transition in Work Teams: Toward a New Model of Group Development. Academy of Management Journal , 31 (1), 9-41.

Gherardi, S., \& Nicolini, D. (2002). Learning in a constellation of interconnected practices: canon or dissonance? Journal of Management Studies , 39 (4), 419-436.

Gianfermi, R., \& Buchholz, S. (2011). Exploring the relationship between job satisfaction and nursing group outcome attainment capability in nurse administrators. Journal of Nursing Management , 19 (8), 1012-1019. 
Gibson, C., \& Early, C. (2007). Collective cognition in action: Accumulation, interaction, examination, and accomodation in the development and operation of group efficacy beliefs in the workplace. Acadamy of Management Review , 32 (2), 438-458.

Glendon, A., \& Stanton, N. (2000). Perspectives on safety culture. Safety Science , 34, 193214.

Glick. (1985). Conceptualizing and measuring organizational and psychological climate: pitfalls in multilevel research. Academy of Management Review , 10 (3), 601-616.

Grant, A. (2008). Does intrinsic motivation fuel the prosocial fire? Motivational synergy in predicting persistence, performance, and productivity. Journal of applied psychology , 93 (48).

Griffin, M., \& Neal, A. (2000). Perceptions of Safety at Work: A Framework for Linking Safety Climate to Safety Performance, Knowledge, and Motivation. Journal of Occupational Health Psychology, 5, 347-358.

Grindle, A., Dickinson, A., \& Boettcher, W. (2000). Behavioral Safety Research in Manufacturing Settings: A Review of the Literature. Journal of Organizational Behavior Management, 20, 29-68.

Grote, G. (2012). Safety management in different high-risk domains - All the same? Safety Science, 50, 1983-1992.

Guldenmund, F. (2010). Misunderstanding Safety Culture and its Relationship to Safety Management. Risk Analysis, 30 (10), 1466-1480.

Guldenmund, F. (2000). The nature of safety culture: a review of theory and research. Safety Science, 34, 215-257.

Hale, A. (2000). Culture's confusions. Safety Science, 34, 1-14.

Haslam, A., Powell, C., \& Turner, J. (2000). Social Identity, Self categorization, and work motivation: rethinking the contribution of the group to positive and sustainable organisational outcomes. Applied Psychology: An International Review , 49 (3), 319-339.

Haslam, S. (2004). Psychology in organizations: The social identity approach (2nd edition). Thousand Oaks, CA: Sage.

Hitlin, S., \& Piliavin, J. (2004). VALUES: Reviving a Dormant Concept. Annual Review of Sociology, 30, 359-393. 
Hofmann, D., \& Morgeson, F. (1999). Safety-related behavior as a social exchange: The role of perceived organizational support and leader-member exchange. Journal of Applied Psychology , 84, 286-296.

Hofmann, D., \& Stetzer, A. (1996). A cross-level investigation of factors influencing unsafe behaviors and accidents. Personnel Psychology , 49, 307-339.

Hofmann, D., Morgeson, F., \& Gerras, S. (2003). Climate as a moderator of the relationship between leader-member exchange and content specific citizenship: Safety climate as an exemplar. Journal of Applied Psychology , 88, 170-178.

Hofstede, G. (1990). Cultures and Organization: Sofware of the Mind. London: McGrawHill.

Hogan, S., \& Coote, L. (2014). Organizational culture, innovation, and performance: A test of Schein's model. Journal of Business Research , 67 (8), 1609-1621.

Hogg, M. (2000). Subjective uncertainty reduction through self-categorization: a motivational theory of social identity processes. European Review of Social Psychology, 11, 223-255.

Hogg, M., \& Terry, D. (2000). Social identity and self-categorization processes in organizational contexts. Academy of Management Review , 25 (1), 121-140.

Høivik, D., Moen, B., Mearns, K., \& Haukelid, K. (2009). An explorative study of health, safety and environment culture in a Norwegian petroleum company. Safety Science , 47 (7), 992-1001.

Holstein, J., \& Gubrium, J. (1995). The Active Interview. Thousand Oaks: Sage.

Holyfield, L., \& Jonas, L. (2003). From river god to research grunt: Identity, emotions, and the river guide. Symbolic Interaction , 26 (2), 285-306.

Hunter, I. (2007). An analysis of whitewater rafting safety data: risk management for programme organizers. Journal of Adventure Education \& Outdoor Learning , 7 (1), 21-35.

IAEA, I. A. (1991). Safety Culture. A report by the International Nuclear Safety Advisory Group. Vienna: IAEA.

Jackson, J. (1975). Normative power and conflict potential. Sociological Methods and Research , 4, 237-263. 
Jia, G. (1998). Quantitative analysis of psychological personality for NPP operators. Nuclear Power Engineering , 19 (2), 168-173.

Jiang, L., Yuc, G., Li, Y., \& Li, F. (2010). Perceived colleagues' safety knowledge/behavior and safety performance: Safety climate as a moderator in a multilevel study. Accident Analysis and Prevention, 42, 1468-1476.

Johnson, H., Singh, A., \& Young, R. (1998). Fall protection analysis for workers on residential roofs. Journal of Construction Engineering and Management , 124 (5), 418428.

Johnson, S., \& Hall, A. (2005). The prediction of safe-lifting behavior: an application of the theory of planned behavior. Journal of Safety Research , 36 (1), 63-73.

Judge, T., \& Kammeyer-Mueller, J. (2012). Job Attitudes. Annual Review of Psychology, $63,341-67$.

Judge, T., Piccolo, R., Podsakoff, N., Shaw, J., \& Rich, B. (2010). The relationship between pay and job satisfaction: A meta-analysis of the literature. Journal of Vocational Behavior , $77(2), 157-167$.

Judge, T., Thoresen, C., Bono, J., \& Patton, G. (2001). The Job Satisfaction-Job Performance Relationship: A Qualitative and Quantitative Review. Psychological Bulletin , 127 (3), 376-407.

Kanfer, R., \& Ackerman, P. (1989). Motivation and cognitive abilities: An integrative/aptitude-treatment interaction approach to skill acquisition. Journal of Applied Psychology, 74, 657-690.

Kanfer, R., Chen, G., \& Pritchard, R. (2008). Work Motivation; Past, Present, and Future. New York: Routledge.

Kaplan, S., Bradley, J., Luchman, J., \& Haynes, D. (2009). On the Role of Positive and Negative Affectivity in Job Performance: A Meta-Analytic Investigation. Journal of Applied Psychology, 94 (1), 162-176.

Karoly, P. (1993). Mechanisms of self-regulation: a systems view. Annual Review of Psychology , 44, 23-52.

Klein, H., Austin, J., \& Cooper, J. (2008). Goal choice and decision processes. In K. e. al, Work Motivation, past, present and future (pp. 101-138). New York: Routledge. 
Kosmowski, K. (2014). Human factors in designing the instrumentation and control systems important to safety. International Journal of Performability Engineering , 741753.

Krosnick, J. e. (1993). Attitude Strength: One Construct or Many Related Constructs? . Journal of Personality and Social Psychology .

Laitinen, H., \& Ruohomäki, I. (1996). The effects of feedback and goal setting on safety performance at two construction sites. Safety Science, 24 (1), 61-73.

Langley, A. (1999). Strategies for theorizing from process data. Academy of Management Review , 24 (4), 691-710.

Lapinski, M., \& Rimal, R. (2005). An explication of social norms. . Communication Theory, $15,127-147$.

Latane, B. (1981). The Psychology of Social Impact. AMERICAN PSYCHOLOGIST, 36 (4), 343-356.

Latham, G. (2012). Work Motivation; History, theory, research and practice (2nd ed.). Los Angeles: Sage.

Latham, G., \& Pinder, C. (2005). Work Motivation Theory and Research at the Dawn of the Twenty-First Century. Annual Review of Psychology , 486-506.

Latimer, A., \& Ginis, K. (2005). The importance of subjective norms for people who care what others think of them. Psychology \& Health , 20 (1), 53-62.

Laurence, D. (2005). Safety rules and regulations on mine sites - The problem and a solution. Journal of Safety Research , 36 (1), 39-50.

Lauver, K., \& Kristof-Brown, A. (2001). Distinguishing between employees' perception of person-job person-organization fit. Journal of Vocational Behaviour , 59, 454-470.

Le, et al. (2011). Too Much of a Good Thing:Curvilinear Relationships Between Personality Traits and Job Performance. Journal of Applied Psychology, 96 (1), 113-133.

Leach, C. W., Van Zomeren, M., Zebel, S., Vliek, M. L., Pennekamp, S. F., Doosje, B., ... \& Spears, R. (2008). Group-level self-definition and self-investment: a hierarchical (multicomponent) model of in-group identification. Journal of personality and social psychology, 95(1), 144. 
Lee, T., \& Harrison, K. (2000). Assessing safety culture in nuclear power stations. Safety Science, 34, 61-97.

Lewin, K., Lippitt, R., \& White, R. (1939). Patterns of aggressive behaviour in experimentally created social climates. Journal of Social Psychology, 10, 271-299.

Lingard, H., \& Holmes, N. (2001). Understanding of occupational health and safety risk control in small business construction. Construction Management Economics , 19 (2), 217226.

Lingard, H., Cooke, T., \& Blismas, N. (2011). Coworkers' response to occupational health and safety. Engineering, Construction and Architectural Management, 18 (2), 159-175.

Lipponen, J., Bardi, A., \& Haapamaki, J. (2008). The interaction between values and organizational identification in predicting suggestion-making at work. Journal of Occupational and Organizational Psychology , 81 (2), 241-248.

Locke, E. (1969). What is job satisfaction? Organizational Behavior and Human Performance , 4 (4), 309-336.

Locke, E., \& Latham, G. (2004). What should we do about motivation theory? Six recommendations for the twenty-first century. Academy of Management Review , 29 (3), 388-403.

Lofquist, E. (2010). The art of measuring nothing: the paradox of measuring safety in a changing civil aviation industry using traditional safety metrics. Safety Science , 48, 15201529.

Lopez de Castro, B., Gracia, F., Peiro, J., Pietrantoni, L., \& Hernandez, A. (2013). Testing the validity of the International Atomic Energy Agency (IAEA) safety culture model. Accident Analysis and Prevention, 60, 231- 244.

Luria, G. (2008). Climate strength: How leaders form consensus. Leadership Quarterly , $19,42-53$.

Luria, G., \& Yagil, D. (2010). Safety perception referents of permanent and temporary employees: Safety climate boundaries in the industrial workplace. Accident Analysis and Prevention , 42, 1423-1430.

MacCurdy, J. T. (1943). The structure of morale. Cambridge University Press. 
Manning, M. (2011). When We Do What We See: The Moderating Role of Social Motivation on the Relation Between Subjective Norms and Behavior in the Theory of Planned Behavior. BASIC AND APPLIED SOCIAL PSYCHOLOGY , 33, 351-364.

Marrelli, A. (2007). Unobtrusive Measures. Performance Improvement , 46 (9), 43-47.

Marsical, M., Garcia Herraro, S., \& Toca Otero, A. (2012). Assessing safety culture in the Spanish nuclear industry through the use of working groups. Safety Science, 50, 12371246.

Martínez-Córcoles, M., Gracia, F., Tomás, I., \& Peiro, J. (2014). Strengthening Safety Compliance in Nuclear Power Operations: A Role-Based Approach. Risk Analysis , 34 (7), 1257-1269.

Maslow, A. (1943). A theory of human motivation. Psychological Review , 50, 370-396.

Masson, T., \& Fritsche, I. (2014). Adherence to climate-change related ingroup norms: Do dimensions of group identification matter? European Journal of Social Psychology, 44, 455-465.

Matheson, C. (2012). The Motivation of Public Sector Employees: An Outline of Six Orientations to Work. Administration \& Society , 44 (2), 207-237.

McClelland, D. (1961). The achieving society. Princeton: Van Nostrand.

McCracken, G. (1988). The Long Interview. Newbury Park: Sage.

McGrath, J. (1981). Dilemmatics: The study of research choices and dilemmas. American Behavioral Scientist, 25 (2), 179-210.

McGrath, J. (1964). Toward a "theory of method" for research on organizations. In W. Cooper, H. Leavitt, \& M. Shelley, New perspectives in organization research (pp. 533-547). New York: Wiley.

McLain, D. L. (2014). Sensitivity to social information, social referencing, and safety attitudes in a hazardous occupation. Journal of occupational health psychology , 19 (4), 425-436.

Mearns, K., \& Flin, R. (1999). Assessing the State of Organizational Safety - Culture or Climate? Current Psychology, 18 (1), 5-17.

Meliá, J. (1998). Un modelo causal psicosocial de los accidentes laborales (A psychosocial causal model of work related accidents). Anuario de Psicología , 29 (3), 25-43. 
Meliá, J., Mearns, K., Silva, S., \& Lima, M. (2008). Safety climate responses and the perceived risk of accidents in the construction industry. Safety Science , 46, 949-958.

Meyer, J., \& Rowan , B. (1977). Institutionalized Organizations: Formal Structure as Myth and Ceremony. American Journal of Sociology , 83, 340-63.

Miles, M., \& Huberman, A. (1984). Qualitatitive Data Analysis, A Sourcebook of New Methods. Newbury Park: Sage.

Miles, M., Huberman, A., \& Saldana, J. (2014). Qualitatitive Data Analysis, A Sourcebook of New Methods. Newbury Park: Sage.

Mischel, W. (1977). The interaction of the person and the situation. In M. a. Endler, Personality at the Crossroads: current issues in interactional psychology. New Jersey: Lawrence Earlbaum.

Moon, H., \& Livne, E. (2011). The past and future of personality utility: Improving predictive ability through item-based precision and factor interdependence. Human Resources Management Review, 21, 258-267.

Moore-Merrell, L., Zhou, A., McDonald-Valentine, S., Goldstein, R., \& Slocum, C. (2008). Contributing Factors To Firefighter Line-Of-Duty Injury In Metropolitan Fire Departments in the United States. United States Fire Association.

Mooren, L., Grzebieta, R., Williamson, A., \& Olivier, J. (2014). Safety management for heavy vehicle transport: A review of the literature. Safety Science , 62, 79-89.

Moray, N., \& Huey, B. (1988). Human factors research and nuclear safety. Washington: National Academy Press.

Moreland, R., \& Zajonc, R. (1982). Exposure Effects in Person Perception: Familiarity, Similarity, and Attraction. Journal of Experimental Social Psychology , 18, 395-415.

Morrow, S., Koves, K., \& Barnes, V. (2014). Exploring the relationship between safety culture and safety performance in U.S. nuclear power operations. Safety Science , 69, 3747.

Mullen, J. (2004). Investigating factors that influence individual safety behavior at work. Journal of Safety Research, 35, 275- 285.

Mullen, J., Kelloway, E., \& Teed, M. (2011). Inconsistent style of leadership as a predictor of safety behaviour. Work and Stress, 25, 41-54. 
Murninghan, K., \& Conlon, D. (1991). The dynamics of intense work groups: A study of British string quartets. Administrative Science Quarterly , 36, 165-186.

Myers, M. (2009). Qualitative Research in Business Management. Thousand Oaks: Sage.

Nahrgang, J., Morgeson, F., \& Hofmann, D. (2008). Predicting safety performance: a metaanalysis of safety and organizational constructs. Presented at the Annual Meeting of the Society for Industrial and Organizational Psychology. San Francisco.

Nahrgang, J., Morgeson, F., \& Hofmann, D. (2011). Safety at Work: A Meta-Analytic Investigation of the Link Between Job Demands, Job Resources, Burnout, Engagement, and Safety Outcomes. Journal of Applied Psychology, 96 (1), 71-94.

Neal, A., \& Griffin, M. (2006). A Study of the Lagged Relationships Among Safety Climate, Safety Motivation, Safety Behavior, and Accidents at the Individual and Group Levels. Journal of Applied Psychology, 91 (4), 946-953.

Neal, A., \& Griffin, M. (2004). Safety climate and safety at work. In Barling \& Frone (Eds.), The psychology of workplace safety (pp. 15-34). Washington, DC: American Psychological Association.

Ogbonna, E., \& Wilkinson, B. (2003). The False Promise of Organizational Culture Change: A Case Study of Middle Managers in Grocery Retailing. Journal of Management Studies, 40 (5), 1151-78.

Omodei, M., McLennan, J., \& Reynolds, C. (2005). Identifying why even well-trained firefighters make unsafe decisions: a human factors interview protocol. International Association of Wildland Fire.

Ontario (1990). Occupational Health and Safety Act, RSO 1990, c 0.1

Ostrom, L., Wilhelmsen, C., \& Kaplan, B. (1993). Assessing safety culture. Nuclear Safety, 34 (2), 163-172.

Parks, L., \& Guay, R. (2009). Personality, values and motivation. Personality and Individual Differences , 47, 675-684.

Patterson, B., Bradley, M., \& Artiss, W. (2000). Operator error and emotions. Operator error and emotions - a major cause of human failure. Proceedings of the Canadian Nuclear Society 21st annual conference. Toronto: Canadian Nuclear Society.

Perrow, C. (1999). Normal Accidents. Princeton: Princeton University Press. 
Pessemier, W. (2008). Developing a safety culture in the fire service. INTERNATIONAL FIRE SERVICE , 2 (1), 7-17.

Pessemier, W., \& England, R. (2012). Safety culture in the US fire service: an empirical definition. International Journal of Emergency Services , 1 (1), 10-28.

Petriglieri, J. (2011). UNDER THREAT: RESPONSES TO AND THE CONSEQUENCES OF THREATS TO INDIVIDUALS' IDENTITIES. ACADEMY OF MANAGEMENT REVIEW , 36 (4), 641-662.

Pidgeon, N. (1998). Safety Culture: key theoretical issues. Work \& Stress , 12 (3), 202-216.

Pinder, C. (2008). Work Motivation in Organizational Behaviour. New York: Psychology Press.

Ployhart, R. (2008). The Measurement and analysis of motivation. In K. e. al., Work Motivation, past, present and future (pp. 18-57). New York: Routledge.

Pratt, M. (2009). For the lack of Boilerplate: Tips on writing up (and reviewing) qualitative research. Academy of Management Journal , 52 (5), 856-862.

Pratt, M. G., Rockmann, K. W., \& Kaufmann, J. B. (2006). Constructing professional identity: The role of work and identity learning cycles in the customization of identity among medical residents. Academy of management journal, 49(2), 235-262.

Preston, J. e. (2011). Action Embellishment: An Intention Bias in the Perception of Success. Journal of Personality and Social Psychology, 101 (2), 233-244.

Probst, T., \& Brubaker, T. (2001). The effects of job insecurity on employee safety outcomes: Cross sectional and longitudinal explorations. Journal of Occupational Health Psychology , 6, 139-159.

Rasmussen, J. (1997). Risk Management in dynamic society: a modelling problem. Safety Science , 27 (2/3), 183-213.

Reason, J. (1998). Achieving a safe culture: theory and practice. Work \& Stress , 12 (3), 293-306.

Reason, J. (1990). Human error. New York: Cambridge University Press.

Reason, J. (1997). Managing the Risks of Organizational Accidents. Surrey: Ashgate.

Reason, J. T. (2001). Understanding adverse events: the human factor. In C. Vincent (Ed.), Clinical risk management. Enhancing patient safety (2 ed., pp. 9--30). London: BMJ Books. 
Reber, R., \& Wallin, J. (1984). The Effects of Training, Goal Setting, and Knowledge of Results on Safe Behavior: A Component Analysis. The Academy of Management Journal, 27 (3), 544-560.

Regehr, C., \& Millar, D. (2007). Situation critical: High demand, low control, and low support in paramedic organizations. Traumatology, 13(1), 49.

Rich, B., Lepine, J., \& Crawford, E. (2010). Job Engagement: Antecedents and Effects on Job Performance. Academy of Management Journal , 53 (3), 617-635.

Richter, A., \& Koch, C. (2004). Integration, differentiation and ambiguity in safety cultures. Safety Science, 42, 703-722.

Rokeach, M. (1973). The Nature of Human Values. New York: Free Press.

Ross, L., \& Nisbett, R. E. (1991). The person and the situation. Perspectives of social psychology. New York: McGraw Hill.

Rosso, B., Dekas, K., \& Wrzesniewski, A. (2010). On the meaning of work: A theoretical integration and review. Research in Organizational Behavior, 30, 91-127.

Ryan, R., \& Deci, E. (2000). Self-determination theory and the facilitation of intrinsic motivation, social development, and well-being. American Psychologist, 55, 68-78.

Salancik, G., \& Pfeffer, J. (1978). A social information processing approach to job attitudes and task design. Administrative Science Quarterly, 23 (2), 224-253.

Salgado, J. (2013). Conscientiousness, its facets, and the prediction of job performance ratings: Evidence against the narrow measures. International Journal of Selection and Assessment, 21 (1), 74-84.

Sampson, J., DeArmond, S., \& Chen, P. (2014). Role of safety stressors and social support on safety performance. Safety Science, 64, 137-145.

Sanders, M., \& McCormick, E. (1987). Human factors in engineering and design. New York: McGRAW-HILL.

Scandura, T., \& Williams, E. (2000). Research Methodology in Management: current practices, trends, adn implications for future research. Academy of Management Journal , 43 (6), 1248-1264.

Schein. (1985). Organizational Culture and Leadership. San Francisco: Jossey Bass. 
Schleicher, D., Watt, J., \& Greguras, G. (2004). Reexamining the job satisfaction and performance relationship. Journal of Applied Psychology , 89 (1), 165-177.

Schneider, B., Brief, A., \& Guzzo, R. (1996). Creating Climate and Culture for Sustainable Organizational Change. Organizational Dynamics , 24 (4), 6-19.

Schwartz, S., \& Boehnke, K. (2004). Evaluating the structure of human values with confirmatory factor analysis. Journal of Research in Personality , 38, 230-255.

Scott-Parker, B., Hyde, M., Watson, B., \& King, M. (2012). "They're lunatics on the road": Exploring the normative influences of parents, friends, and police on young novices' risky driving decisions. Safety Science , 50 (9), 1917-1928.

Shamir, B. (1991). Meaning, self and motivation in organizations. Organization Studies, $12(3), 405-424$.

Shamir, B., House, R., \& Arthur, M. (1993). The Motivational Effects of Charismatic Leadership: A Self-Concept Based Theory. Organization science , 4 (4), 577-594.

Sharpe, R. (2005). "Going above and beyond": The emotional labor of adventure guides. Journal of Leisure Research, 37, 29-50.

Sheeran, P. (2002). Intention-Behavior Relations: A Conceptual and Empirical Review. European Review of Social Psychology, 12, 1-36.

Shields, A., \& Flin, R. (2012). Paramedics' non-technical skills: a literature review. Emergency Medicine Journal, emermed-2012.

Silbey, S. (2009). Taming prometheus: Talk about safety and culture. . Annual Review of Sociology, 35, 341-369.

Singleton, R., \& Straits, B. (2010). Approaches to social research (5th ed.). Oxford, UK: Oxford University Press.

Skinner, B. (1953). Science and human behaviour. New York: Macmillan.

Smith, D., \& DeJoy, M. (2014). Safety climate, safety behaviors and line-of-duty injuries in the fire service. International Journal of Emergency Services , 3 (1), 49-64.

Sorensen, J. (2002). Safety culture: a survey of the state-of-the-art. Reliability Engineering \& System Safety, 76 (2), 189-204.

Spiggle, S. (1994). Analysis and Interpretation of Qualitative Data in Consumer Research. Journal of Consumer Research , 21, 491-503. 
Stark, E., Shaw, J., \& Duffy, M. (2007). Preference for group work, winning orientation, and social loafing behaviour in groups. Group and Organization Management, 32 (6), 699723.

Steers, R., Mowday, R., \& Shapiro, D. (2004). The Future of Work Motivation Theory. Academy of Management Review , 379-387.

Strack, F., \& Deutsch, R. (2004). Reflective and Impulsive Determinants of Social Behavior. Personality and Social Psychology Review , 8 (3), 220-247.

Stride et al. (2013). Negative safety events as correlates of work-safety tension. Safety Science, 53, 45-50.

Sulzer-Azaroff, B., \& Austin, J. (2000). Does BBS Work? Behavior-based Safety and Injury Reduction: A Survey of the Evidence. Professional Safety, 45, A Survey of the Evidence,' Professional Safety, 45, 19-24.

Sutton, R., \& Staw, B. (1995). What theory is not. Administrative Science Quarterly , 40 (3), 371-384.

Tajfel, H. (1974). Social identity and intergroup behaviour. Social Science Information , 14, 65-93.

Tavares, W., Bowles, R., \& Donelon, B. (2016). Informing a Canadian paramedic profile: framing concepts, roles and crosscutting themes. BMC Health Services Research, 16(1), 477.

Thackaberry, J. (2004). "Discursive Opening" and Closing in Organisational Self-Study Culture as Trap and Tool in Wildland Firefighting Safety. Management Communication Quarterly, 17 (3), 319-359.

Toode, K., Suominen, T., \& Routasalo, P. (2011). Work motivation of nurses: A literature review. International Journal of Nursing Studies , 48 (2), 246-257.

Törner, M. (2011). The "social-physiology" of safety. An integrative approach to understanding organisational psychological mechanisms behind safety performance. Safety Science , 49, 1262-1269.

Townley, B. (1997). The Institutional Logic of Performance Appraisal. Organization Studies , 18, 261-85. 
Tucker et al. (2008). Perceived Organizational Support for Safety and Employee Safety Voice: The mediating role of coworker support for safety. Journal of Occupational Health Psychology, 13 (4), 319-330.

Turner, N., \& Gray, G. (2009). Socially constructing safety. Human Relations, 62 (9), 12591266.

US Nuclear Regulatory Commission. (2000). Technical basis and implementation guidelines for a technique for human event analysis (ATHEANA). US Nuclear Regulatory Commission.

Van Dijk, D., \& Kluger, A. (2004). Feedback sign effect on motivation: Is it moderated by regulatory focus? Applied Psychology: In international review , 53, 113-135.

Van Eerde, W., \& Thierry, H. (1996). Vroom's expectancy models and work-related criteria: a meta-analysis. Journal of Applied Psychology, 81, 575-586.

Van Kleef, G., Van Doorn, E., Heerdink, M., \& Koning, L. (2011). Emotion is for influence. EUROPEAN REVIEW OF SOCIAL PSYCHOLOGY , 22, 114-163.

Vaughn. (1996). The Challenger Launch Decision: Risky Technology, Culture, and Deviance at NASA. Chicago: University of Chicago Press.

Vidmar, N., \& Rokeach, M. (1974). Archie Bunker's Bigotry: A Study in Selective Perception and Exposure. Journal of Communication , 24 (1), 36-47.

Vinodkumara, M., \& Bhasib, M. (2010). Safety management practices and safety behaviour: Assessing the mediating role of safety knowledge and motivation. Accident Analysis and Prevention , 42, 2082-2093.

Vroom, V. (1964). Work and Motivation. New York: Wiley.

Wallace, J., \& Chen, G. (2006). A multilevel integration of personality, climate, selfregulation, and performance. . Personnel Psychology, 59, 529-557.

Weick, K. (2010). Reflections on Enacted Sensemaking in the Bhopal Disaster. Journal of Management Studies , 47 (3), 537-550.

Weick, K. (1993). The collapse of sensemaking in organizations: The Mann Gulch disaster. Administrative Science Quarterly , 38, 628-652.

Weick, K., Sutcliffe, K., \& Obsfeld, D. (1999). Organizing for High Reliability: Processes of Collective Mindfulness. Research in Organizational Behavior , 1, 81-123. 
Weick, K., Sutcliffe, K., \& Obstfeld, D. (2005). Organizing and the Process of Sensemaking. Organization Science, 16 (4), 409-421.

Westaby, J., \& Lowe, J. (2005). Risk-taking orientation and injury among youth workers: examining the social influence of supervisors, coworkers and parents. Journal of Applied Psychology, 90, 1027-35.

Whisman, S., \& Hollenhorst, S. (1999). Injuries in commercial whitewater rafting. Clinical Journal of Sport Medicine, 9 (1), 18-23.

Wilde, G. (1982). The Theory of Risk Homeostasis: Implications for Safety and Health. Risk Analysis , 2, 209-225.

Wilkins, A. L., \& Ouchi, W. G. (1983). Efficient cultures: Exploring the relationship between culture and organizational performance. Administrative science quarterly, 468-481.

Wilmott. (1993). Strength is Ignorance; Slavery is Freedom: Managing Culture in Modern Organizations. Journal of Management Studies, 30, 515-52.

Wilson, T., Lindsay, S., \& Schooler, T. (2000). A Model of Dual Attitudes. Psychological Review , 107 (1), 101-126.

Wright, B., \& Pandey, S. (2008). Public service motivation and the assumption of personorganization fit: Testing the mediating effect of value congruence. Administration and Society, 40 (5), 502-521.

Xenikou, A., \& Furnham, A. (1996). A correlational and factor analysis study of four questionnaire measures of organizational culture. Human Relations, 49, 349-371.

Xu, e. a. (2014). Attentional bias toward safety predicts safety behaviors. Accident Analysis \& Prevention, 71, 144-153.

Yagil, D., \& Luria, G. (2010). Friends in Need: The Protective Effect of Social Relationships Under Low-Safety Climate. Group \& Organization Management , 35 (6), 727-750.

Yauch, C., \& Steudel, H. (2003). Complementary use of qualitative and quantitative cultural assessment methods. Organizational Research Methods , 6, 465-481.

Zhang, H. (2002). Safety Culture: a concept in chaos. Human Factors and Ergonomics .

Zhou, Q., Fang, D., \& Wang, X. (2008). A method to identify strategies for the improvement of human safety behavior by considering safety climate and personal experience. Safety Science, 46, 1406-19. 
Zohar, D. (2000). A group-level model of safety climate: testing the effect of group climate on microaccidents in manufacturing jobs. Journal of Applied Psychology, 85 (4), 587-596.

Zohar, D. (1980). Safety Climate in Industrial Organizations: Theoretical and Applied Implications. Journal of Applied Psychology , 65, 96-102.

Zohar, D. (2010). Thirty years of safety climate research: Reflections and future directions. Accident Analysis and Prevention , 42, 1517-1522.

Zohar, D., \& Fussfeld, N. (1981). Modifying Earplug Wearing Behavior by Behavior Modification Techniques: An Empirical Evaluation. Journal of Organizational Behavior Management , 3 (2), 41-52.

Zohar, D., \& Luria, G. (2005). A Multilevel Model of Safety Climate: Cross-Level Relationships Between Organization and Group-Level Climates. Journal of Applied Psychology , 90 (4), 616-628.

Zohar, D., \& Luria, G. (2004). Climate as a Social-cognitive Construction of Supervisory Safety Practices: Scripts as Proxy of Behavior Patterns. Journal of Applied Psychology, 89, 322-333.

Zohar, D., \& Polachek, T. (2014). Discourse-based intervention for modifying supervisory communication as leverage for safety climate and performance improvement: A randomized field study. Journal of Applied Psychology , 99 (1), 113-124. 


\section{APPENDICES}

\section{APPENDIX 1: URBAN FIRE SERVICE EMAIL INVITATION}

Subject line: Invitation to participate in a research project on safety performance

Jeff Jackson is a professor at Algonquin College and is completing a PhD in safety motivation. He would like you to participate in the research he is doing, and asked to have this invitation forwarded to you.

Dear Sir or Madam

My name is Jeff Jackson and I am doing research to complete a PhD at Carleton University's Sprott School of Business (under the supervision of Professor Steven Murphy). I'm studying how individuals who work in 'safety critical jobs' such as yours perceive their coworkers' safety expectations and how that influences their own safety performance. I am inviting you to either an individual interview or to participate in a focus group discussion on this topic. I am looking for your opinion on the importance of safety to you and to your coworkers.

If you volunteer to participate, you will be asked to either have an individual interview or be a part of a group discussion (each approximately 1 hour in time commitment). Your employer has generously agreed to do this during working hours. I will schedule a time that suits you and your employer's needs.

There are no known risks to participating in this research. Participation is completely voluntary and you may choose to withdraw from the research at any time or not answer questions that you do not feel comfortable answering. Should you decide to withdraw, the information you have provided will be destroyed.

Your responses to the questions will be kept confidential. Each interview or discussion participant will be assigned a number code to help ensure that personal identifiers are not revealed during the analysis and report of findings. The interviews and discussions will be audio recorded, password protected, and then destroyed after transcribing. For focus group participants, since we will be talking in a group, I cannot promise that what you say will remain completely private, but I will ask that you and all other group members respect the privacy of everyone in the group.

The ethics protocol for this project was reviewed by the Carleton University Research Ethics Board, which provided clearance to carry out the research. Should you have 
questions or concerns related to your involvement in this research, please contact the REB Chair.

While there is no direct benefit to you for volunteering, your contribution to this emerging field of study will help our understanding of safety performance and have an application to all industries that deal with safety. Your participation is greatly appreciated.

If you would like to participate in this research project, or have any questions, please contact me. I look forward to your participation.

Sincerely,

Jeff Jackson 


\section{APPENDIX 2: INTERVIEW PROTOCOL:}

The interview protocol proceeded as follows (same for both group and individual interviews):

- Introduce researcher and thank the participant for their time. Clarify that the interview will be a maximum of approximately 60 minutes.

- Provide a brief overview of the study.

- Remind informant that participation is completely voluntary and at any point they can choose not to participate. Participants are also not obligated to answer a question if he/she prefers not to.

- Outline that participant responses will not be identified and that steps will be taken to ensure confidentiality. Please omit personal names during discussion of interview questions.

- State that this study is for academic research purposes.

- State that the interview will be audio recorded and that the researcher will take handwritten notes. Audio recordings will be transcribed and (audio files) destroyed. All notes will be secured.

- Address any questions.

- Have participant sign consent form.

- Commence interview discussion.

Upon conclusion of interview: 
- Thank participant(s) for their time.

- Reiterate confidentiality agreement.

-Address any final questions and comments.

- Clarify approval for further contact if clarification is needed on any points made during the interview.

- Provide contact information of researcher.

- Follow-up with a "Thank you" e-mail.

Privacy and confidentiality of the participants are considered at all stages of this interview protocol. 


\section{APPENDIX 3: INTERVIEW QUESTIONS}

What is the influence of coworkers upon individual internalization of safety? In order to address this research question propositions were formed to structure this inquiry. The propositions are deduced from a review of the literature and theoretical connections offered by underutilized (in safety studies) social motivation theories, described in an earlier section of this report. From these propositions, a series of interview questions are assembled to provide insight into the phenomenon under study: What is the influence of coworkers upon individual internalization of safety?

\section{Individual Interviews:}

The individual interviews will focus on four themes, drawn from the guiding propositions:

1. Personal safety values (importance of safety)

2. Internalized versus external motivation for safety performance

3. Aspects of coworker influence

4. Safety in relation to self-concept

In order to draw out informants' perspectives on these themes and to address the research question, the following interview questions will be asked. As this research prescribes to McCracken's (1998) semi-structured approach, open ended clarifying questions will be inserted as needed.

\section{Theme 1: Personal values related to job and safety}

1. What does the term safety mean in your job? 
2. From a safety perspective, what do you believe are the three most important tasks you have to do or ought to do as part of your job?

3. How does safety fit into your organization's responsibilities and priorities? Do you buy into those? Why or why not?

4. How do balance between your own individual safety, your coworker's safety, victim/patient safety and getting the job done?

a. Can you think of a time when this balance was particularly difficult? What did you do?

5. Are there some aspects to safety in your job where you go above and beyond policy - things you choose to do because they make better safety sense, even though you don't have to?

a. How do you justify taking different safety precautions even though you don't have to?

b. Give me an example of when it's ok to break some of those policies

6. Tell me of a time at work when you felt your own safety was at risk. What did you do?

a. Who did you look to for help?

7. Do colleagues feel psychologically safe in your culture to discuss risks and personal experiences or deviations from policy? Give an example.

\section{Theme 2: Aspects of coworker influence}

8. Do you think your coworkers value safety in the same way as you do? Give concrete examples as to why you feel this way.

9. How do you define the 'ideal' worker's safety performance? Would your coworkers have a similar definition? How do you know?

10. Are people assessed on their safety performance? If so, how? If not, why not?

11. Do you trust that your coworkers are performing safely on a day-to-day basis?

12. Do you think your coworkers watch you to see if you are performing safely?

a. How does it make you feel to know you are/aren't watched?

b. What does that say about your coworkers' views on safety? 
13. How do your coworkers deal with someone who is seen as cutting corners on safety?

14. What motivates you to work safely?

a. What do you think motivates your coworkers to perform safely?

15. Think of a coworker who has influenced your safety perspective. What is it about them that made them influential to you?

16. Do you think you have influenced your coworkers' safety beliefs? How?

17. If you find yourself unsure on a tough safety decision, who do you look to for direction? Why that person?

18. Is there peer pressure at work when it comes to safety? Give an example.

a. Is there an example of [opposite] positive/negative peer pressure?

\section{Theme 3 and 4: Internalized motivation and self-concept}

19. Is there a skill set for safety tasks that you are particularly good at or conscientious about compared to your coworkers?

20. Is it important to you that your coworkers think that you are safe? Why or why not?

21. Whose opinion of your safety performance matters most to you? Why?

22. Do you ever consider what your coworkers will think of you if you make a mistake or perform unsafely?

23. I see a difference in some people who view safety as something they have to 'do', versus other people for whom safety is something they believe. Do you see that difference among your coworkers? How can you tell what camp they're in?

\section{Group Interviews:}

1. Do you think your station has a unique perspective on safety, different than other stations in the city? What is that perspective, and why is that?

a. Does your platoon have a unique safety culture compared to other platoons at this station? Why is that?

b. What are some safety expectations that you and your team have that might be specific to your platoon? (Theme 1) 
2. Do you think people at this station agree upon the importance of safety? What gives you that impression? (Theme $1 \& 2$ )

3. Could someone from another platoon join your shift and just slide in when it comes to safety practices, or would there be a transition period? What does it take to transition a new person in to your safety practices? Example?

4. Can you think of an example when there is a difference between the way you are supposed to do things and the way you actually do things? Policy versus practice? (Theme 4)

a. How does the way you 'actually' do a task get passed around?

b. How do you know when it's time to go by the book or follow peer practice? Can you provide examples?

5. Do you and your coworkers talk about safety related issues? What are some of those issues? (Changes in policy vs. internally recognized issues). (Theme $1 \& 3$ )

a. Are these formal or informal conversations?

b. Do these ever turn into changes in adopted practice? Example?

6. How are mistakes or close calls dealt with - internally within your team, not the formal authority version?

7. What happens when someone disagrees with the team on a certain safety issue? What do you do about that? What have you seen others do about that situation? (Theme 1, 3 \& 4)

8. Do your coworkers provide pressure to ensure you perform safely? (Theme 1, 2 \& 3)

a. What happens if you don't pull your weight from a safety perspective?

9. What kind of messages do your higher-ups give you regarding safety? What do you do with those messages?

10. What role does the company officer/station chief play in creating a safety culture? Is it effective?

a. Is it more or less important than what you agree upon as a team?

11. Is safety measured here? Is it rewarded? 
The open-ended nature of these questions was intended to allow the emergence of individual perspectives regarding coworker influence. The discussion unfolded in the informants' own words, based on their own context and experiences. The questions were designed to provide "tentative answers to novel questions [... and suggest] new connections among phenomena" (Edmondson \& McManus, 2007: 1158). The goal was to elicit Miles and Huberman's “thick descriptions that are vivid and nested in a real context, and have a ring of truth that has strong impact on the reader" (Miles, Huberman, \& Saldana, 2014: 10). 


\section{APPENDIX 4: FIRST CYCLE AND SECOND CYCLE CODING CATEGORIES}

\begin{tabular}{|c|c|}
\hline First Cycle Coding Categories & Second Cycle Coding Categories \\
\hline $\begin{array}{l}\text { Safety norms as representative } \\
\text { of agreed upon safety values } \\
\text { (Proposition } 1 \text { ) }\end{array}$ & $\begin{array}{l}\text { - Concertive norms } \\
\text { - Descriptive norms }\end{array}$ \\
\hline $\begin{array}{l}\text { Social identity tied to safety and } \\
\text { group buy in (Proposition 2) }\end{array}$ & [no further category added] \\
\hline $\begin{array}{l}\text { Aspects of coworker influence } \\
\text { upon safety }\end{array}$ & $\begin{array}{l}\text { - Ambiguity reduction } \\
\text { - Need to fit in } \\
\text { - Social support for safety } \\
\text { - We vs. I } \\
\text { - On own - no team }\end{array}$ \\
\hline $\begin{array}{l}\text { Internalized versus external } \\
\text { motivation for } \quad \text { safety } \\
\text { performance (Proposition 3) }\end{array}$ & $\begin{array}{l}\text { - Concrete vs. conceptualized } \\
\text { view of safety } \\
\text { - In-group vs. out-group } \\
\text { - Working in team }\end{array}$ \\
\hline $\begin{array}{lll}\text { Compliance } & \text { versus safety } \\
\text { participation } & & \end{array}$ & $\begin{array}{l}\text { - Individual safety values } \\
\text { - Organization safety values } \\
\text { ○ Org. sensemaking } \\
\circ \quad \text { Production vs. safety }\end{array}$ \\
\hline $\begin{array}{l}\text { Safety in relation to self-concept } \\
\text { (Proposition } 4)\end{array}$ & $\begin{array}{ll}\text { - } & \text { Protect social id (Prop. 5) } \\
\text { - } & \text { Trust }\end{array}$ \\
\hline
\end{tabular}

